Universidad Politécnica de Madrid

Escuela Técnica Superior de Ingenieros de Telecomunicación

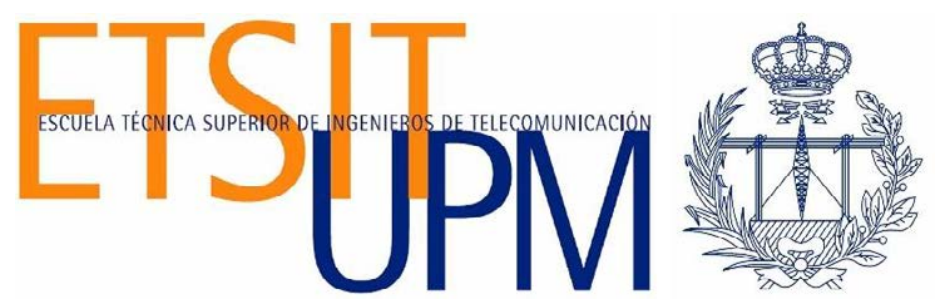

\title{
CONTRIBUCIÓN A LAS ARQUITECTURAS DE VIRTUALIZACIÓN DE FUNCIONES DE RED Y REDES DEFINIDAS POR SOFTWARE APLICADAS A LAS REDES RESIDENCIALES CON GESTIÓN CENTRADA EN EL USUARIO
}

Tesis Doctoral

Jorge Ricardo Flores Moyano

Ingeniero Electrónico

2018 

Departamento de Ingeniería de Sistemas Telemáticos ESCUELA TÉCNICA SUPERIOR DE INGENIEROS DE TELECOMUNICACIÓN UNIVERSIDAD POLITÉCNICA DE MADRID

TESIS DOCTORAL

\title{
CONTRIBUCIÓN A LAS ARQUITECTURAS DE VIRTUALIZACIÓN DE FUNCIONES DE RED Y REDES DEFINIDAS POR SOFTWARE APLICADAS A LAS REDES RESIDENCIALES CON GESTIÓN CENTRADA EN EL USUARIO
}

\author{
Autor \\ Jorge Ricardo Flores Moyano \\ Ingeniero Electrónico
}

\author{
Director \\ David Fernández Cambronero \\ Doctor Ingeniero de Telecomunicación
}

Madrid, abril de 2018 

Título: Contribución a las Arquitecturas de Virtualización de Funciones de Red y Redes Definidas por Software Aplicadas a las Redes Residenciales con Gestión Centrada en el Usuario

Departamento: Departamento de Ingeniería de Sistemas Telemáticos

Autor: $\quad$ Jorge Ricardo Flores Moyano

Director: $\quad$ David Fernández Cambronero

\section{TRIBUNAL CALIFICADOR}

\section{Presidente:}

Vocales:

Secretario:

Suplentes:

Realizado el acto de defensa y lectura de la Tesis Doctoral en Madrid, el día...... de de 2018 

Simplicity is a great virtue but it requires hard work to achieve it and education to appreciate it. And to make matters worse: complexity sells better

- Edsger W. Dijkstra

Become Who Are You by Learning Who You Are -Pindar 

A Verónica, Sofía y mis Padres 



\section{Agradecimientos}

Al finalizar mi etapa como doctorando, me es grato dedicar unas palabras de agradecimiento a las diferentes personas e instituciones que de una $u$ otra forma han contribuido positivamente en la realización de esta investigación. En primer lugar, quisiera agradecer a mi director de tesis, Dr. David Fernández, por su acertada guía y sabios consejos que he recibido durante todos estos años. Su carisma y la confianza depositada han sido valores clave que han mantenido la motivación viva en mí.

En segundo lugar, quisiera agradecer al grupo de investigación GIROS, a los profesores Luis Bellido y Encarna Pastor por sus valiosas sugerencias que han permito mejorar las propuestas que se presentan en esta tesis. Por otra parte, a Miguel y Carlos, con los cuales hemos compartido varios momentos que han permitido que la estancia en la escuela sea más llevadera. Creo que con todos los miembros del grupo más que una relación profesional se ha establecido una amistad que espero conservar durante los años venideros. Adicionalmente, quisiera también agradecer a mis amigos de Ecuador Lorena, César y Danny con quienes de igual manera hemos compartido gratos momentos en nuestra estancia de doctorado.

En tercer lugar, agradecer a la Secretaria de Educación Superior, Ciencia, Tecnología e Innovación de la república del Ecuador por proporcionar la financiación necesaria para la realización de mis estudios de doctorado.

En cuarto lugar, agradecer a mis queridos padres Jorge y Yolanda por el apoyo incondicional y la fe puesta en mí. Me enseñaron a volar, a no rendirme jamás, a ser fuerte en momentos difíciles y a apreciar los momentos que la vida nos da. Hemos caminado siempre juntos a pesar de estar lejos, porque nuestras mentes y corazones mantienen un vínculo único e inquebrantable. Su acompañamiento durante toda mi vida ha permitido que logre cumplir este objetivo y que a la vez representa un logro para ustedes también.

Finalmente, de manera especial, gracias infinitas a los tesoros más preciados de mi vida, mi esposa Verónica y mi hija Sofía. Juntos empezamos una travesía con incertidumbre y miedo, pero con la esperanza de que el sacrificio realizado nos proporcionará un futuro mejor. De esta experiencia llegó a nuestras vidas Sofía, como un rayo de luz a iluminar nuestro vivir. Decirte a ti Verónica que en todos estos años has sido un soporte fundamental para realizar y finalmente culminar esta tesis, sin ti esto no hubiera sido posible. Y a ti pequeña Sofía decirte que en los últimos meses tu cálida sonrisa al llegar a casa han sido el mejor regalo que la vida me ha dado; te amo hija mía, alegría de mi vida.

Sin extenderme más quisiera decir que la realización de una tesis doctoral es un proceso complejo que al final proporciona alegrías y penas. Forma el carácter, y pone a prueba nuestra fortaleza y madurez. El conocimiento adquirido es muy valioso, pero talvez más valioso es el crecimiento como persona que se experimenta. 



\section{Abstract}

This dissertation addresses three specific problems found nowadays in residential networks. Firstly, the restricted usability that residential networks offer due to the fact that a technical knowledge, which the majority of users lack, is required to exert management tasks. Secondly, the high degree of heterogeneity of the residential environment in terms of user profiles, end devices and network technologies. And thirdly, the limited flexibility of current network equipment to improve the connectivity service provided by the residential network, which makes it difficult to add new network functionalities required by today's multimedia services. According to the state of the art, the problems described above are the result of the Internet-like network model established for the residential network, which relies on a good level of technical expertise of administrators. Added to this is the lack of flexibility that current networking model presents to evolve a network service and adapt it to the dynamic, demanding and diverse requirements of, for example, multimedia applications and enterprise network services.

In this context, the first contribution of this dissertation is the design of a new management architecture for residential networks that takes advantage of the new paradigms of Network Functions Virtualization (NFV) and Software Defined Networks (SDN) to allow using a user-centric approach and to facilitate its implementation. In this architecture, users actively participate in the management tasks of their own networks by using Residential Network Management applications, referred to as "RENEMA apps". These apps, which are adapted to the skills of an average user, allow customizing the network based on the user's preferences, improving the usability of the network and facing the high degree of heterogeneity that the residential environment presents. On the other hand, to facilitate the implementation of the usercentric approach, the architecture provides different Residential Network Services (RENESEs) to abstract the low-level network configuration and monitoring. Thus, RENEMA apps developers would not have to cope with them.

The second contribution of this dissertation is a differentiated traffic treatment proposal based on NFV's Service Function Chaining (SFC) functionality, that enriches the connectivity service provided by the residential network. The differentiated traffic treatment allows the residential network to accomplish the divergent requirements of applications and services such as video on demand or online games, so they can achieve an optimal performance. For this purpose, the management architecture coordinates the collaborative operation of the SDN and NFV components in such a way that their individual contributions are complemented. In this sense, the SDN-based residential gateway deployed in the user's premises is in charge of differentiating the traffic while the NFV component is in charge of deploying the SFCs that will process the previously differentiated traffic at the ISP's cloud computing infrastructure.

To validate the proposals presented, a use case and its corresponding proof of concept has been developed. This has required the design and implementation of a validation scenario composed of the residential network, the access network and the cloud computing infrastructure of the ISP. The validation scenario includes two virtual testbeds in which functional, performance and QoS provision tests have been carried out. The results obtained have allowed verifying the feasibility and the benefits that the proposed management architecture provides. Moreover, to validate the creation of the virtualized Management and Networking Domain (vMANDO) as an ETSI-NFV Network Service (NS), the NFV profile developed by TOSCA has been used to obtain the corresponding deployment templates. As a result of this exercise, some limitations of TOSCA regarding Network Services life cycle management have been identified.

Keywords: Residential network, User-centric, Residential Network Management application, Residential Network Services, Virtualized Management and Networking Domain, Network Functions Virtualization, Software Defined Networks, Differentiated Traffic Treatment. 


\section{Resumen}

La presente tesis doctoral aborda tres problemas puntuales que se encuentran hoy en día en las redes residenciales. En primer lugar, la usabilidad restringida que presentan las redes residenciales debido a que se requieren conocimientos técnicos para ejercer las tareas de gestión, los cuales son inexistentes en la mayoría de usuarios. En segundo lugar, el alto grado de heterogeneidad del entorno residencial en cuanto a perfiles de usuario, dispositivos finales y tecnologías de red. Y, en tercer lugar, la flexibilidad limitada de los equipos de red actuales para mejorar el servicio de conectividad proporcionado por la red residencial, lo cual dificulta agregar nuevas funcionalidades de red requeridas por los servicios multimedia de hoy en día. De acuerdo con el estado del arte, los problemas descritos anteriormente son el resultado de haber establecido en el entorno residencial un modelo de red al estilo de Internet en el cual es necesario garantizar el conocimiento y la experiencia técnica. A esto se suma la falta de flexibilidad que presenta el modelo de red actual para permitir que un servicio de red evolucione y se adapte a los requisitos dinámicos, exigentes y diversos de, por ejemplo, aplicaciones multimedia y servicios de red empresariales.

En este contexto, la primera contribución de la presente tesis doctoral consiste en el diseño de una nueva arquitectura de gestión para las redes residenciales que aprovecha los paradigmas de Virtualización de Funciones de Red (NFV) y Redes Definidas por Software (SDN) para permitir el uso de un enfoque centrado en el usuario y facilitar su implementación. En esta arquitectura, los usuarios participan activamente en las tareas de gestión de sus propias redes mediante el uso de aplicaciones para la Gestión de la Red Residencial, denominadas como "aplicaciones RENEMA". Estas aplicaciones, las cuales están adaptadas a las habilidades de un usuario promedio, permiten personalizar la red según las preferencias del usuario, mejorar la usabilidad de la red y enfrentar el alto grado de heterogeneidad que presenta el entorno residencial. Por otra parte, para facilitar la implementación del enfoque centrado en el usuario, la arquitectura proporciona diferentes Servicios de Red Residencial (RENESE) para abstraer la configuración y monitorización de red de bajo nivel. De esta manera, los desarrolladores de aplicaciones RENEMA no tendrían que preocuparse de estas tareas de bajo nivel.

La segunda contribución de la presente tesis doctoral consiste en una propuesta de tratamiento de tráfico diferenciado basada en la funcionalidad de Encadenamiento de Función de Servicio (SFC) de NFV, destinada a enriquecer el servicio de conectividad proporcionado por la red residencial. El tratamiento de tráfico diferenciado permite que la red residencial cumpla con los requisitos divergentes de aplicaciones y servicios, como el video bajo demanda o juegos en línea, para que puedan lograr un rendimiento óptimo. Para este fin, la arquitectura de gestión coordina la operación colaborativa de los componentes SDN y NFV de tal manera que sus contribuciones individuales se complementan. En este sentido, el gateway residencial basado en SDN que se despliega en las instalaciones del usuario se encarga de diferenciar el tráfico, mientras que el componente NFV se encarga de implementar las SFCs que procesarán el tráfico previamente diferenciado en la infraestructura de computación en la Nube del ISP.

Para validar las propuestas presentadas, se ha desarrollado un caso de uso y su correspondiente prueba de concepto. Esto ha requerido el diseño e implementación de un escenario de validación compuesto por la red residencial, la red de acceso y la infraestructura de computación en la Nube del ISP. El escenario de validación incluye dos bancos de prueba virtual en los que se han llevado a cabo pruebas funcionales, de rendimiento y de provisión de QoS. Los resultados obtenidos han permitido verificar la factibilidad y los beneficios que brinda la arquitectura de gestión propuesta. Además, para validar la creación del Dominio de Gestión y Redes virtualizado (vMANDO) como un Servicio de Red (NS) en el contexto de ETSI-NFV, se ha utilizado el perfil NFV desarrollado por TOSCA para obtener las plantillas de despliegue correspondientes. Como resultado de este ejercicio, se han identificado algunas limitaciones de TOSCA con respecto al manejo del ciclo de vida de Servicios de Red. 
Palabras Clave: Red residencial, Centrado en el usuario, aplicación para la Gestión de la Red Residencial, Servicios de Red Residencial, Dominio de Gestión y Redes virtualizado, Virtualización de Funciones de Red, Redes Definidas por Software, Tratamiento de Tráfico Diferenciado. 


\section{Índice de Contenidos}

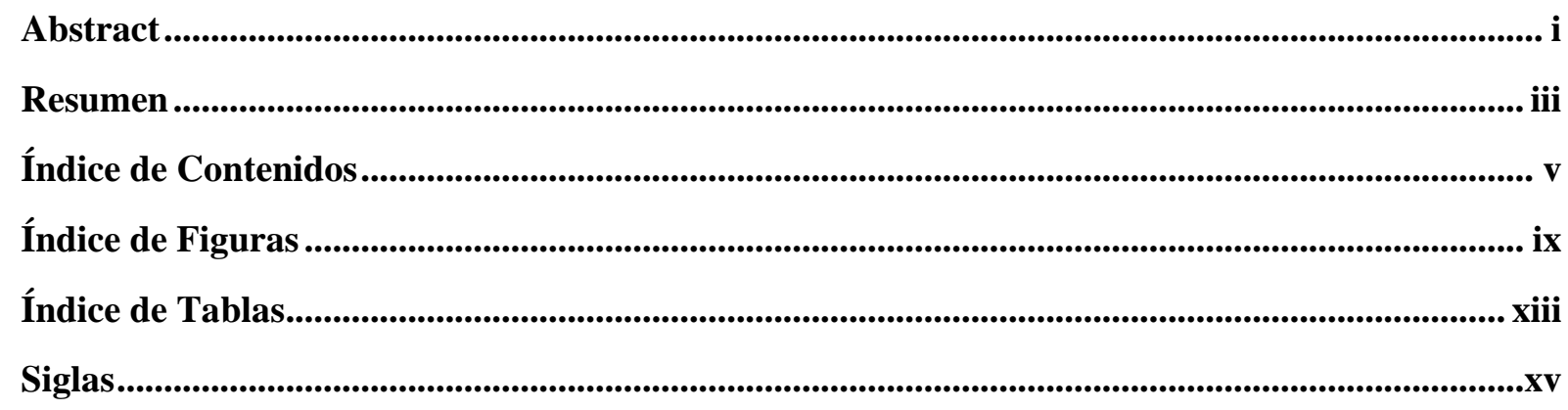

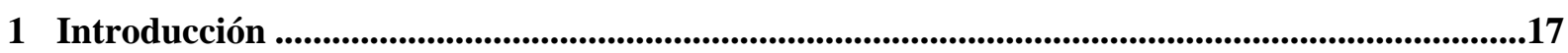

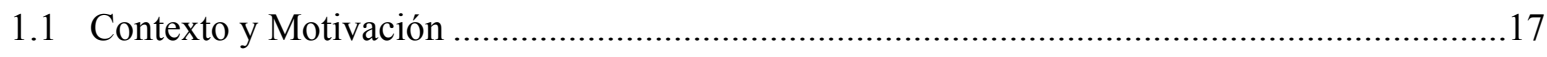

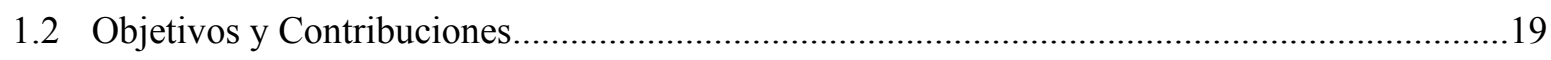

1.3 Metodología de Trabajo y Estructura de la Memoria ................................................................21

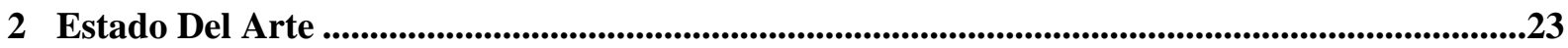

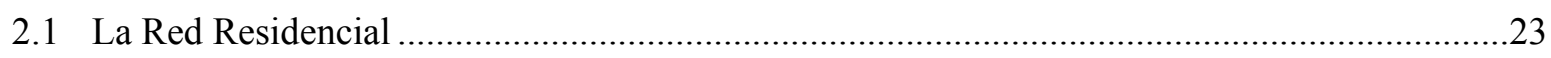

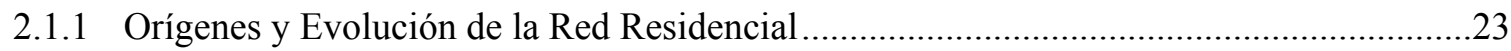

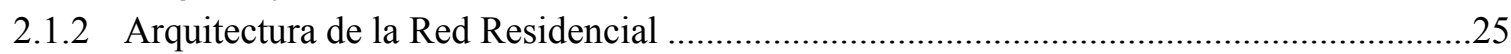

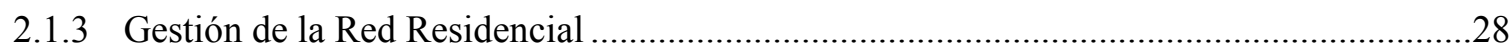

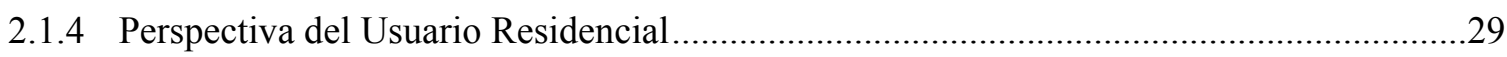

2.2 Conceptos Generales sobre Redes Definidas por Software ..................................................... 31

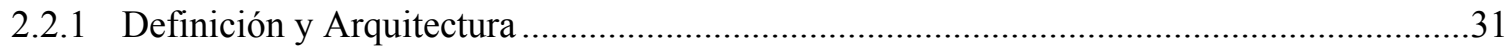

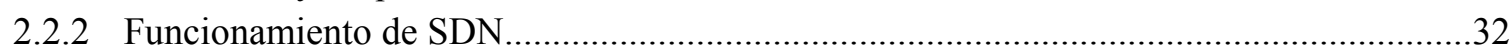

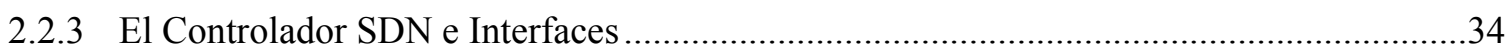

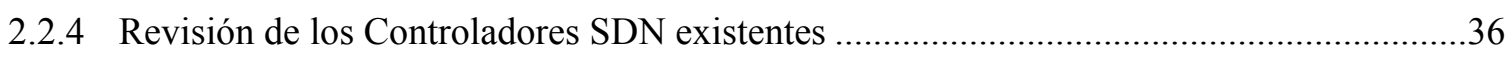

2.3 Conceptos Generales sobre Virtualización de Funciones de Red .............................................38

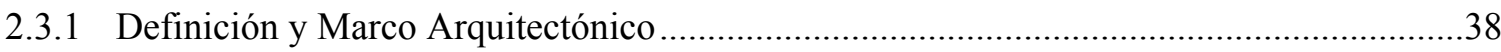

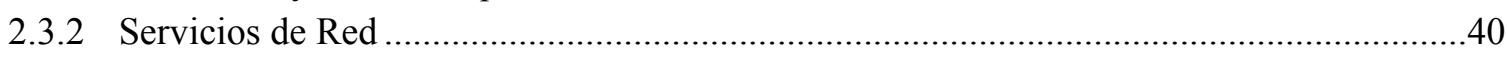

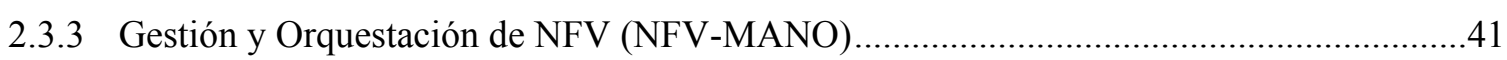

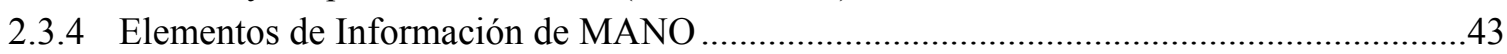

2.3.5 Interfaces NFV-MANO para la Gestión y Despliegue de Servicios de Red .......................48

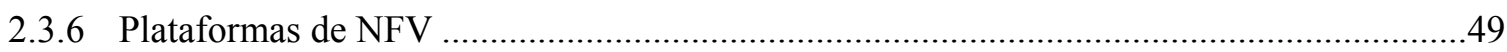

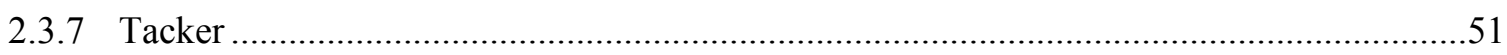

2.4 SDN, NFV y Virtualización Aplicadas a las Redes Residenciales............................................54

2.4.1 Virtualización y Virtualización de Funciones de Red (NFV) ..............................................5

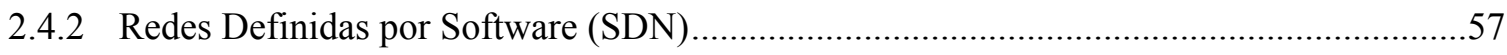

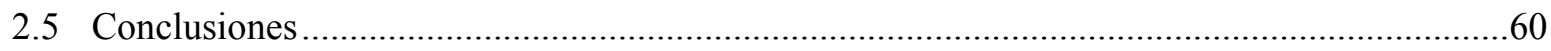

3 Arquitectura de Gestión Centrada en el Usuario para Redes Residenciales...............................63

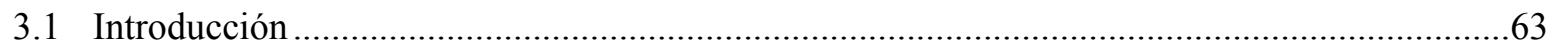


3.2 Diseño de la Arquitectura de Gestión Centrada en el Usuario ................................................. 64

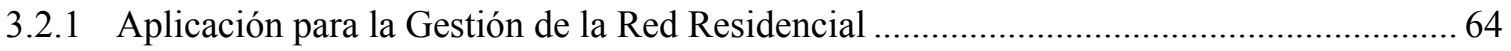

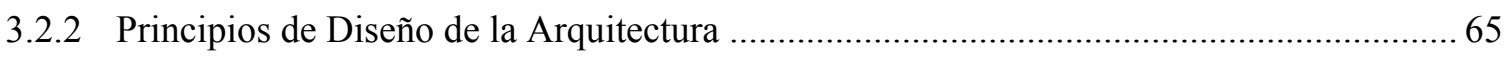

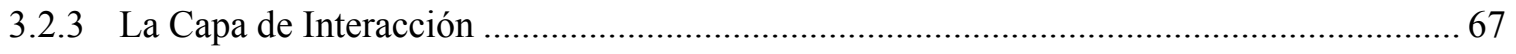

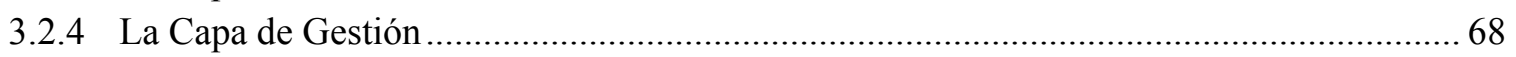

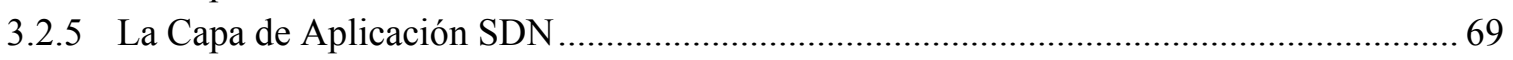

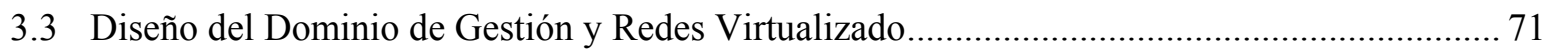

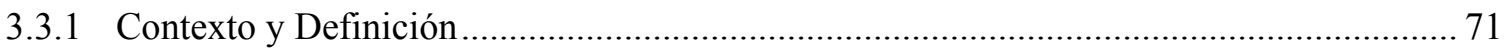

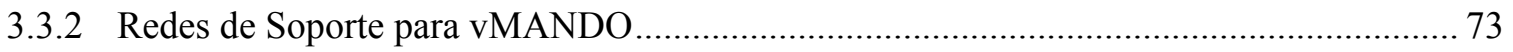

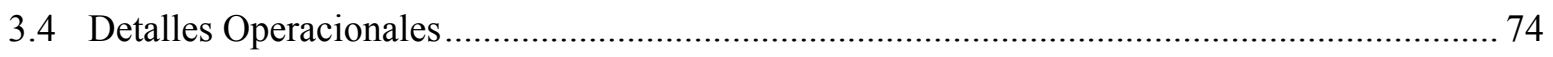

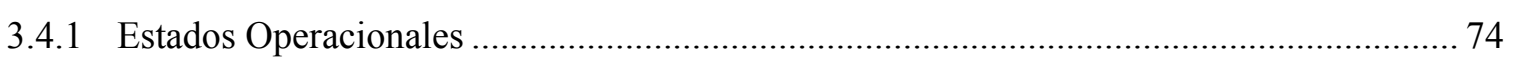

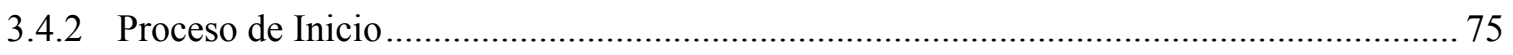

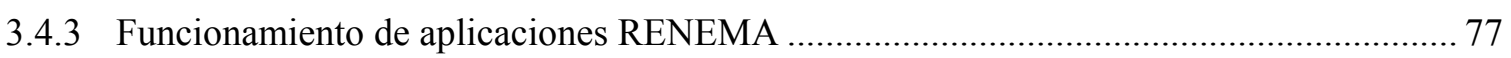

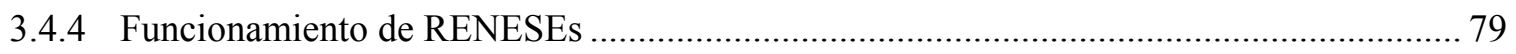

3.4.5 Participación de Gestores en el Intercambio de Información de Gestión ............................ 80

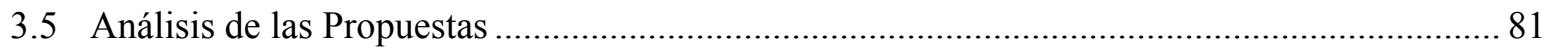

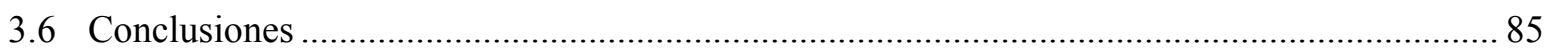

4 Tratamiento de Tráfico Diferenciado basado en SDN y NFV .......................................................... 87

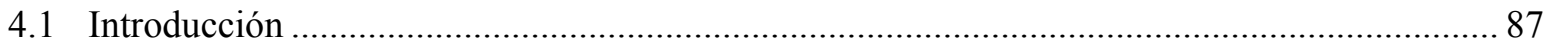

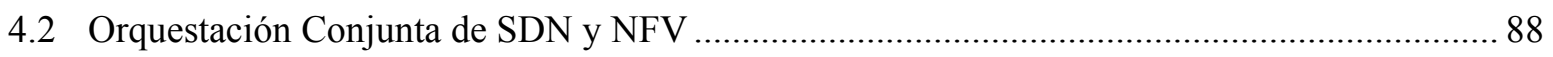

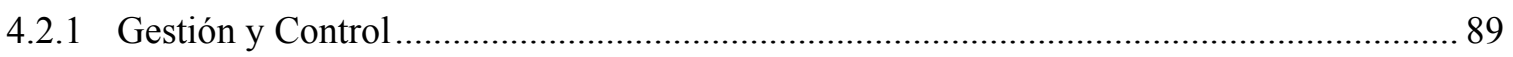

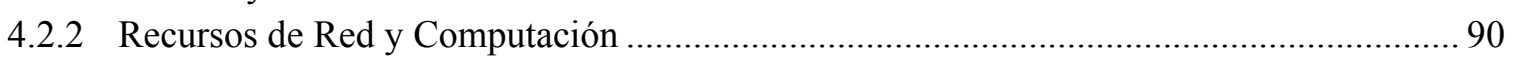

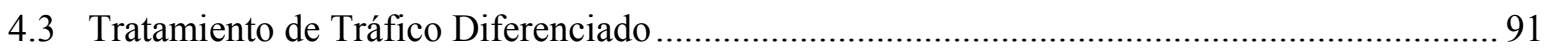

4.3.1 Estructura Interna de vMANDO para Soportar el Tratamiento de Tráfico Diferenciado.. 92

4.4 Aplicación RENEMA para el Tratamiento de Tráfico Diferenciado ........................................ 94

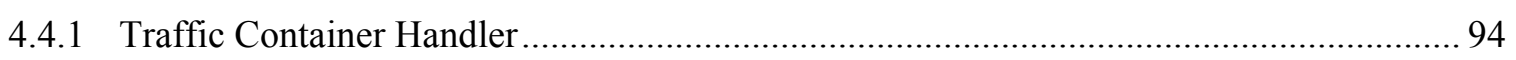

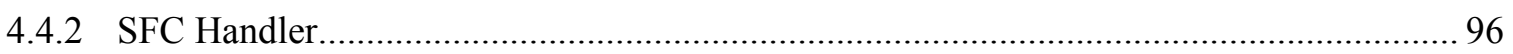

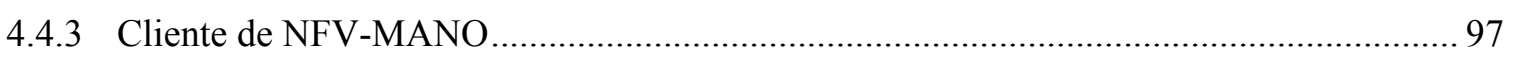

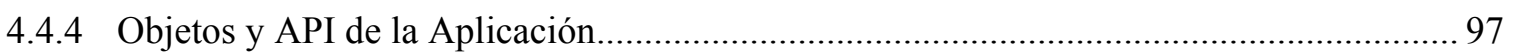

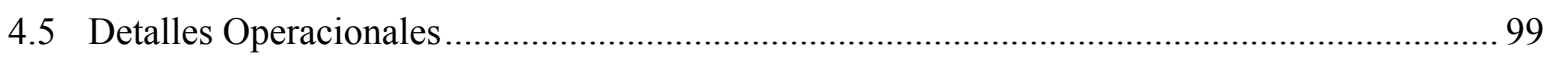

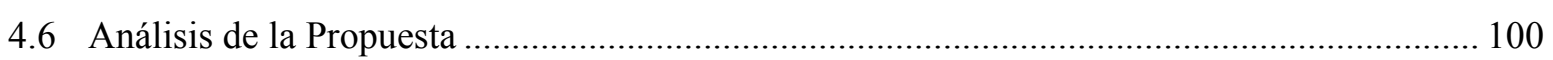

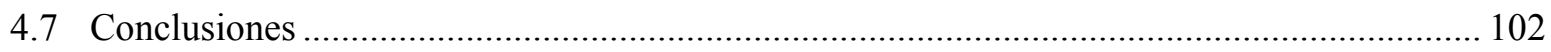

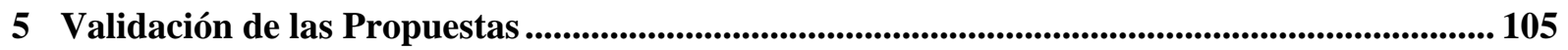

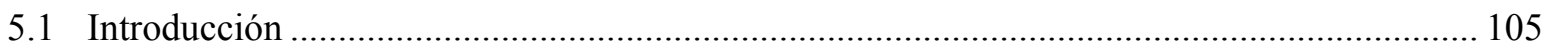

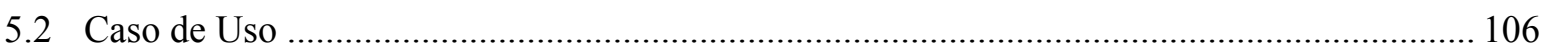

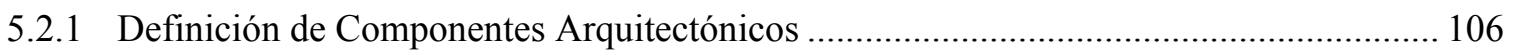

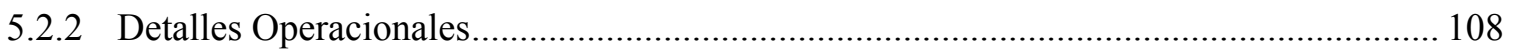

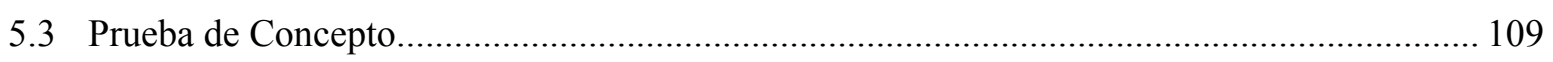

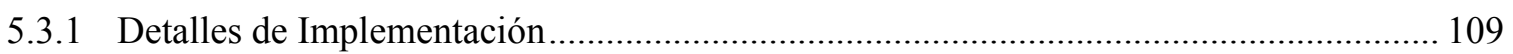

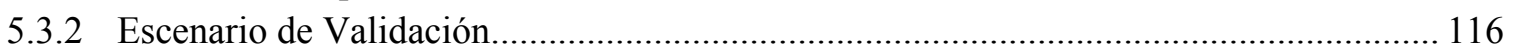

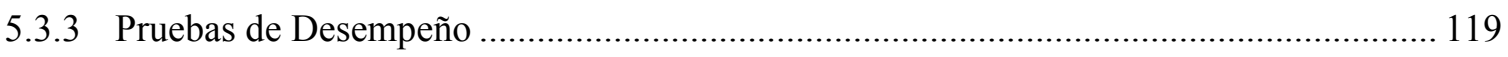

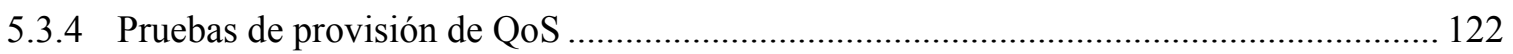


5.4 Implementación del Tratamiento de Tráfico Diferenciado ......................................................124

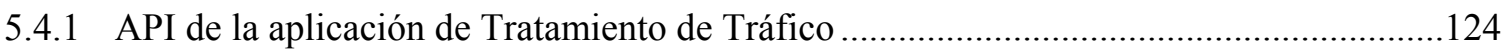

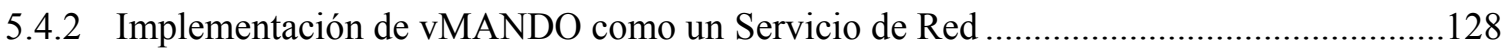

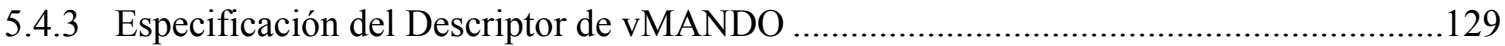

5.4.4 TOSCA y Tacker en la Implementación del Tratamiento de Tráfico Diferenciado ..........134

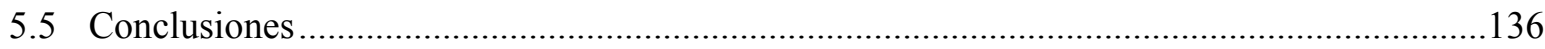

6 Conclusiones y Trabajos Futuros ...........................................................................................................139

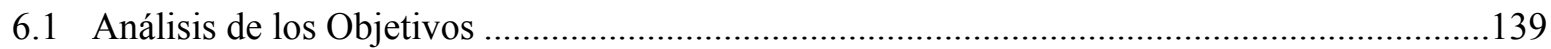

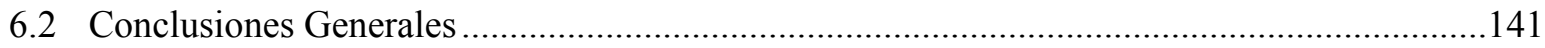

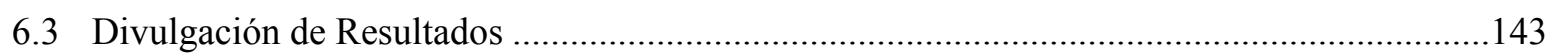

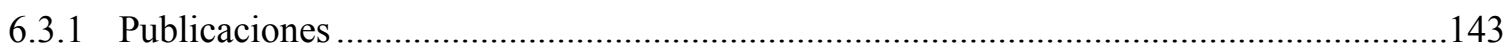

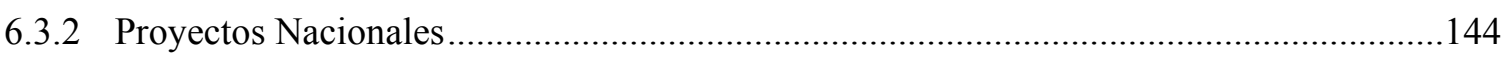

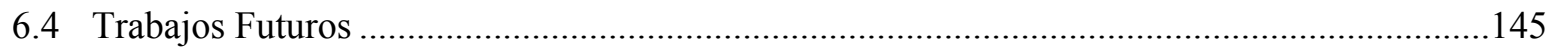

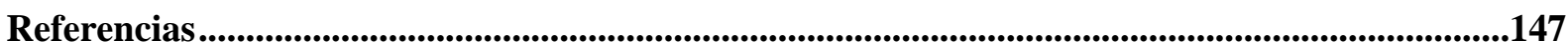

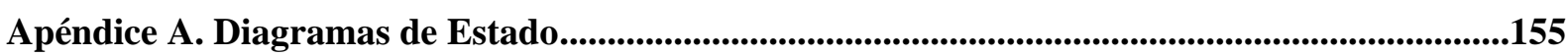

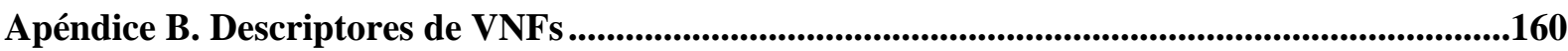




\section{Índice de Figuras}

Figura 2.1 Arquitectura simplificada de una red residencial [8]. Un dispositivo de compartición (RN Sharing Device) permite interconectar diferentes dispositivos para formar la red residencial. A su vez, el módem de banda ancha (BB) conecta la red residencial a la red

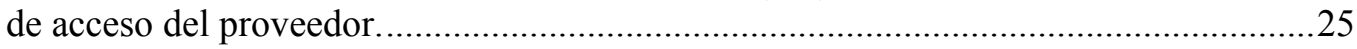

Figura 2.2 Arquitectura funcional para la red residencial propuesta por Broadband Forum [8] ....26

Figura 2.3 Diagrama conceptual del modelo en capas propuesto por ITU-T para la red residencial

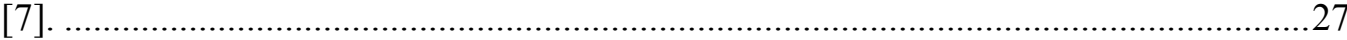

Figura 2.4 Modelo de gestión para la red residencial propuesto por Broadband Forum [8]...........28

Figura 2.5 Arquitectura SDN [22]. El concepto de plano de datos en el contexto de la arquitectura SDN incluye funciones de procesamiento y envío de tráfico. El plano de datos puede incluir el subconjunto mínimo necesario de funciones de control y gestión. ...................32

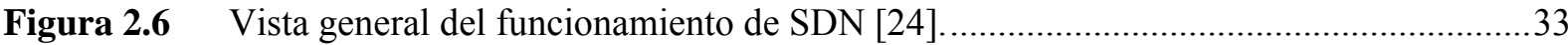

Figura 2.7 Diagrama de flujo del procesamiento de paquetes que realiza un dispositivo OpenFlow

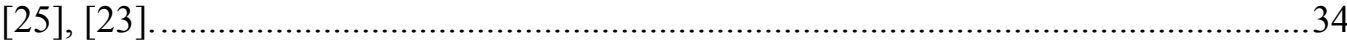

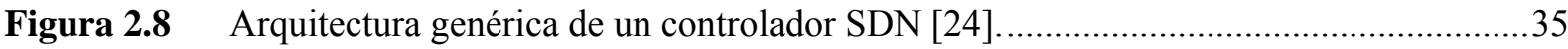

Figura 2.9 Interfaz Northbound basada en "propósitos" o "intents". Representa un paso hacia la creación de un API que elimina la necesidad de conocer y comprender los detalles de los dispositivos en la red física [24].

Figura 2.10 Paradigma NFV. Con este nuevo paradigma, las funciones de red pueden implementarse en cualquier hardware genérico que ofrezca los recursos básicos para procesamiento, almacenamiento y transmisión de datos [31] ................................................................39

Figura 2.11 Marco arquitectónico de referencia para NFV [39] .....................................................40

Figura 2.12 Diferentes grafos de envío de VNF pueden ser definidos sobre el mismo grafo de topología de conectividad de red [42] .......................................................................

Figura 2.13 Marco arquitectónico de NFV-MANO con puntos de referencia [42] .........................42

Figura 2.14 Elementos de información: (a) Estructura de alto nivel, (b) Distribución en catálogos y registros [42].

Figura 2.15 Plantilla de servicio de TOSCA: (a) Elementos y las relaciones entre ellos, (b) Establecimiento de la correspondencia entre la plantilla de servicio definida en TOSCA y el descriptor de servicio de red definido en NFV [40].

Figura 2.16 Descripción de un servicio de red mediante el perfil NFV de TOSCA. Los enlaces virtuales (VLs) requeridos por VNF1 son proporcionados por NSD mediante un proceso de substitución. Todos los archivos de descripción utilizados tienen la extensión yaml.

Figura 2.17 Arquitectura de alto nivel de Tacker.

Figura 2.18 Flujo de trabajo de Tacker. Las operaciones CRUD de descriptores e instancias desplegadas son controladas mediante los APIs NFVO NS, VNFM y VNFFG. ............54

Figura 3.1 Enfoque de alto nivel centrado en el usuario. Las aplicaciones RENEMA brindan a los usuarios residenciales la capacidad de configurar diferentes parámetros de la red........65 
Figura 3.2 Arquitectura de gestión de referencia centrada en el usuario. La centralidad en el usuario se consigue al colocar las capas de Interacción y de Gestión en la parte superior de la arquitectura base SDN. Estas capas adicionales forman juntas una aplicación RENEMA. Nótese cómo diferentes capas se ejecutan en diferentes ubicaciones.

Figura 3.3 Bloques funcionales internos organizados en diferentes capas. Nótese cómo la comunicación entre capas se realiza a través de APIs específicas. La capa de Interacción se ejecuta en los dispositivos preferidos del usuario residencial mientras que el resto de capas de software se ejecutan en las instalaciones del ISP. 68

Figura 3.4 Modelo de interacción entre aplicaciones RENEMA y RENESEs. Ambos gestores ayudan en el intercambio de información de gestión utilizando para ello el protocolo RENESE. En la parte superior de la figura, se proporciona un ejemplo de un mensaje que utiliza un formato JSON. El servicio de Seguimiento de Dispositivos notifica una "conexión de dispositivo" a las aplicaciones suscritas. 70

Figura 3.5 Estructura de vMANDO y su disposición en las instalaciones del ISP. Se crea un vMANDO por red residencial para alojar a la Arquitectura de Gestión SDN centrada en

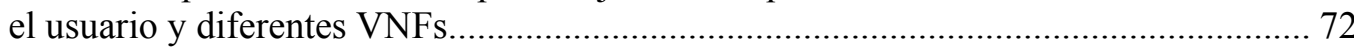

Figura 3.6 Estados operacionales de aplicaciones RENEMA y RENESEs. …............................... 75

Figura 3.7 Diagrama de estados del proceso de inicio de una aplicación RENEMA. .................... 76

Figura 3.8 Diagrama de estados del proceso de Inicio de un Servicio de Red Residencial (RENESE).

Figura 3.9 Diagrama de estados del funcionamiento de una aplicación de acuerdo con el procesamiento de solicitudes realizadas al API REST. ................................................. 78

Figura 3.10 Diagrama de estados del funcionamiento de una aplicación de acuerdo con el procesamiento de mensajes RENESE...................................................................... 78

Figura 3.11 Diagrama de estados del funcionamiento de un RENESE de acuerdo con el

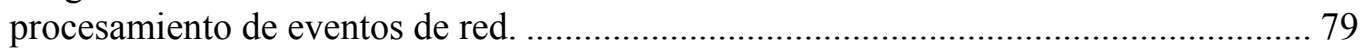

Figura 3.12 Diagrama de estados del funcionamiento de un RENESE de acuerdo con el

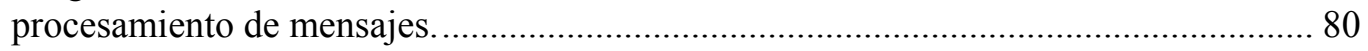

Figura 3.13 Participación de los Gestores de las capas de Gestión y Aplicación SDN en la coordinación del intercambio de información de gestión. .81

Figura 4.1 Orquestación conjunta de SDN y NFV: (a) El sistema de gestión interactúa con el sistema de control de la Red de Acceso Óptica Definida por Software y con la Gestión y Orquestación de NFV para coordinar el funcionamiento de los componentes SDN y NFV. (b) Componentes de Gestión y Red que forman parte de vMANDO. 89

Figura 4.2 Tratamiento de tráfico diferenciado. Se definen diferentes flujos de tráfico a ser agrupados en contenedores de tráfico. Posteriormente, cada contenedor es asociado a una SFC específica de acuerdo con la estrategia de procesamiento de tráfico o el nivel de QoS solicitado.

Figura 4.3 Por medio del uso de etiquetas VLAN, se divide la interfaz WAN de la red residencial en varios canales virtuales que permiten transportar y dirigir los flujos de tráfico a su correspondiente SFC. 92

Figura 4.4 Estructura interna de vMANDO. La Gestión y Orquestación (MANO) de NFV está a cargo de instanciar diferentes VNFs para crear una SFC específica de acuerdo con las especificaciones recibidas desde el Sistema de Gestión Centrado en el Usuario. 93

Figura 4.5 Ejemplo de tratamiento de tráfico diferenciado con calidad de servicio (QoS). Las SFCs $A$ y $B$ son asignadas a los contenedores de tráfico 1 y 2 respectivamente. 94 
Figura 4.6 Aplicación de Tratamiento de Tráfico. Los bloques funcionales de la aplicación utilizan las APIs proporcionadas por NFV-MANO para controlar los procesos CRUD de SFCs en la NFVI y el protocolo RENESE para controlar los procesos CRUD de contenedores de tráfico en el RGW basado en SDN.

Figura 4.7 Objetos definidos para el tratamiento de tráfico diferenciado y la relación existente entre los mismos.

Figura 4.8 Proceso de implementación del tratamiento de tráfico diferenciado. La aplicación se comunica con el servicio de Conmutación mediante el protocolo RENESE. Por otra parte, la aplicación utiliza los servicios que proporciona NFV-MANO a través de las interfaces $N S$ y $V N F$. Con el fin de simplificar el gráfico, se ha omitido el cliente MANO de la Capa de Gestión. 100

Figura 5.1 Interacción de los componentes arquitectónicos en el caso de uso. Nótese cómo los diferentes componentes back-end de las aplicaciones RENEMA interactúan con diferentes servicios a través del protocolo RENESE (intercambio de mensajes). Los componentes back-end y front-end interactúan entre sí a través del API REST. Con el objetivo de simplificar el gráfico, se han omitido ambos gestores. 108

Figura 5.2 Diagrama de estado de la aplicación de Control Parental para procesar solicitudes realizadas al API REST.

Figura 5.3 Diagrama de estado de la aplicación de Control Parental para procesar mensajes

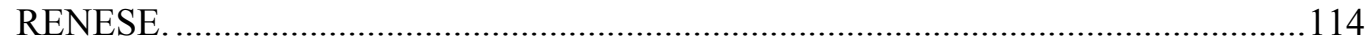

Figura 5.4 Diagrama de estado del servicio de Conmutación para procesar Packet-Ins................115

Figura 5.5 Diagrama de estado del servicio de Conmutación para procesar mensajes RENESE..115

Figura 5.6 Escenario de validación propuesto. Nótese como se integran la red residencial, la red de acceso y la NFVI ubicada en la central de telecomunicaciones del ISP (Central Office). Para simplificar el gráfico no se ha incluido el componente de gestión y control.........116

Figura 5.7 Primera versión del banco de pruebas virtual desarrollado para la prueba de concepto. Para emular la Nube del ISP se utiliza principalmente máquinas virtuales. 117

Figura 5.8 Segunda versión del banco de pruebas virtual desarrollado para la prueba de concepto. La Nube del ISP se sustenta principalmente en OpenStack. 118

Figura 5.9 Intervalos de tiempo que intervienen en la instalación de una entrada de flujo. La sumatoria de estos intervalos permite obtener el retraso en la instalación de la entrada de flujo.

Figura 5.10 El retraso en la instalación de la entrada de flujo como una función de la carga de tráfico. Para congestionar la red de acceso se ejecuta una conexión iperf por host conectado. 121

Figura 5.11 Comportamiento del retraso en la instalación de la entrada de flujo utilizando canales virtuales. La mejora es notable ya que la latencia de la red de acceso solamente afecta a $\beta_{1}$ y el retraso total permanece constante a medida que aumenta la carga de tráfico...122

Figura 5.12 Variabilidad en la calidad del video en el cliente DASH-JS sin provisión de QoS: (a) Caudal de tráfico y calidades del video medidas, (b) Distribución de las calidades del video durante la prueba.

Figura 5.13 Variabilidad en la calidad del video en el cliente DASH-JS con provisión de QoS. (a) Caudal de tráfico y calidades del video medidas, (b) Distribución de las calidades del

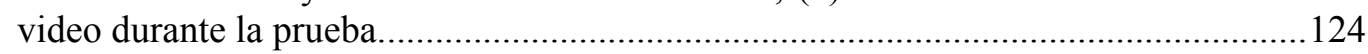

Figura 5.14 Grafo de topología de conectividad de red (NCT) para vMANDO. ............................129

Figura 5.15 Descriptor para vMANDO (vMANDOD) basado en el NSD del perfil NFV de TOSCA. 
Figura 5.16 Descriptor para la VNF Routing + NAT. Se define un nuevo tipo de nodo para la VNF mediante una plantilla de servicio.

Figura 5.17 Descriptor para el VNFFG que especifica la ruta por defecto del tráfico de datos. El descriptor que se muestra está basado en los ejemplos que proporciona Tacker para el VNFFGD.

Figura 5.18 Relación entre las plantillas de servicio utilizadas para describir los componentes de vMANDO.

Figura 5.19 Conjunto de datos ampliado propuesto para vMANDO. (a) Datos adicionales a incluir en la sección "metadata" del descriptor o como un parámetro de solicitud. (b) Datos adicionales a incluir en el campo "attributes" de la instancia. 


\section{Índice de Tablas}

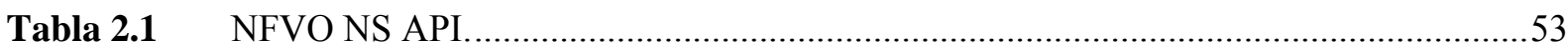

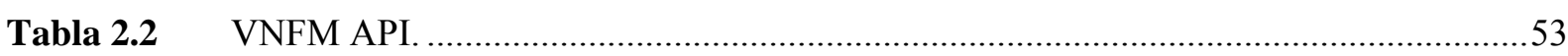

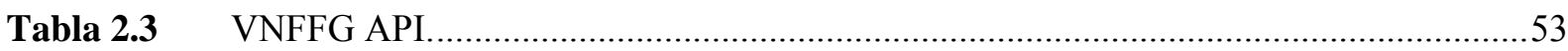

Tabla 4.1 API REST proporcionada por la aplicación de Tratamiento de Tráfico. ........................98

Tabla 5.1 Definición de políticas por medio de la aplicación de Control Parental .....................106

Tabla 5.2 API basada en el intercambio de mensajes RENESE. En la capa de Aplicación SDN, NERON, NESA y los servicios de Conmutación y Seguimiento de Dispositivos proporcionan un conjunto de funciones o API para facilitar la gestión de la red

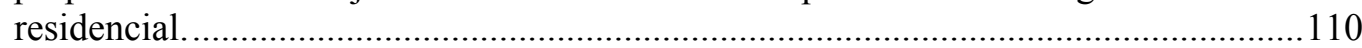

Tabla 5.3 API de las aplicaciones RENEMA. En la Capa de Gestión, las aplicaciones de Control Parental y Estado de la Red exponen sus capacidades de gestión utilizando un API REST. En la capa Interacción, sus respectivos clientes usan esta API para permitir la gestión de la red residencial.

Tabla 5.4 Parámetros de Solicitud y Respuesta del API REST de la aplicación de Control Parental. 112

Tabla 5.5 Parámetros de Solicitud y Respuesta del API REST de la aplicación de Estado de la Red. .113

Tabla 5.6 Servicios proporcionados por el Gestor de aplicación RENEMA y el Gestor de RENESE. Cada tiene su propio prefijo para identificar aplicaciones y servicios.

Tabla 5.7 API REST proporcionada por la aplicación de Tratamiento de Tráfico. El API incorpora operaciones CRUD para flujos de tráfico, contenedores de tráfico, SFCs y VNFs......125

Tabla 5.8 Parámetros de Solicitud y Respuesta del API REST para las operaciones CRUD de flujos de tráfico. 126

Tabla 5.9 Parámetros de Solicitud y Respuesta del API REST para las operaciones CRUD de contenedores de tráfico.

Tabla 5.10 Parámetros de Solicitud y Respuesta del API REST para las operaciones CRUD de SFCs.

Tabla 5.11 Parámetros de Solicitud y Respuesta del API REST para las operaciones CRUD de VNFs. 128

Tabla 5.12 Datos complementarios para definir el modelo de vMANDO. 134 


\section{Siglas}

\begin{tabular}{|c|c|}
\hline API & Application Programming Interface \\
\hline BoD & Bandwidth on Demand \\
\hline BSS & Business Support System \\
\hline CapEx & Capital Expenditure \\
\hline $\mathrm{CO}$ & Central Office \\
\hline CORD & Central Office Re-architected as a Data Center \\
\hline COTS & Commercial Off-The-Shelf \\
\hline $\mathrm{CP}$ & Connection Point \\
\hline $\mathrm{CPE}$ & Customer Premises Equipment \\
\hline CRUD & Create, Read, Update and Delete \\
\hline DB & Date Base \\
\hline DHCP & Dynamic Host Configuration Protocol \\
\hline DNS & Domain Name System \\
\hline EM & Element Management \\
\hline ETSI & European Telecommunications Standards Institute \\
\hline FCAPS & Fault, Configuration, Accounting, Performance and Security \\
\hline FTTH & Fiber To The Home \\
\hline GPON & Gigabit-capable Passive Optical Network \\
\hline $\mathrm{HCI}$ & Human-computer Interaction \\
\hline HTTP & Hypertext Transfer Protocol \\
\hline IETF & Internet Engineering Task Force \\
\hline IoT & Internet of Things \\
\hline IRTF & Internet Research Task Force \\
\hline ISP & Internet Service Provider \\
\hline ITU-T & International Telecommunications Union, Telecommunication Standardization \\
\hline KPI & Key Performance Indicator \\
\hline LAN & Local Area Network \\
\hline LXC & Linux Containers \\
\hline MAC & Media Access Control \\
\hline MANO & Management and Orchestration \\
\hline MIDB & Management Information Data Base \\
\hline NAT & Network Address Translation \\
\hline NBI & Northbound Interface \\
\hline NCT & Network Connectivity Topology \\
\hline NERG & Network Enhanced Residential Gateway \\
\hline NERON & Network Resources Control \\
\hline NESA & Network Statistics \\
\hline NFP & Network Forwarding Path \\
\hline NFV & Network Functions Virtualization \\
\hline NFVI & Network Functions Virtualization Infrastructure \\
\hline NFVO & NFV Orchestrator \\
\hline NS & Network Service \\
\hline NSD & Network Service Descriptor \\
\hline NSR & Network Service Record \\
\hline OLT & Optical Line Terminal \\
\hline ONF & Open Networking Foundation \\
\hline
\end{tabular}




$\begin{array}{ll}\text { ONT } & \text { Optical Network Terminal } \\ \text { OpEx } & \text { Operational Expenditure } \\ \text { OSS } & \text { Operation Support System } \\ \text { OVS } & \text { Open vSwitch } \\ \text { P2P } & \text { Peer-to-peer } \\ \text { PNF } & \text { Physical Network Functions } \\ \text { PNFD } & \text { Physical Network Function Descriptor } \\ \text { PNFR } & \text { Physical Network Function Record } \\ \text { QoE } & \text { Quality of Experience } \\ \text { QoS } & \text { Quality of Service } \\ \text { RENEMA } & \text { Residential Network Management } \\ \text { RENESE } & \text { Residential Network Service } \\ \text { REST } & \text { Representational State Transfer } \\ \text { RGW } & \text { Residential Gateway } \\ \text { RNaaS } & \text { Residential Network as a Service } \\ \text { SBI } & \text { Southbound Interface } \\ \text { SDAN } & \text { Software-Defined Access Network } \\ \text { SDN } & \text { Software-defined Networks } \\ \text { SDO } & \text { Standardization Development Organization } \\ \text { SDOAN } & \text { Software-Defined Optical Access Network } \\ \text { SDOANI } & \text { Software-Defined Optical Access Network Infrastructure } \\ & \text { Sector } \\ \text { SFC } & \text { Service Function Chain } \\ \text { SLA } & \text { Service Level Agreement } \\ \text { TOSCA } & \text { Topology and Orchestration Specification for Cloud Applications } \\ \text { URI } & \text { Uniform Resource Identifier } \\ \text { vCSD } & \text { virtualized Common Services Domain } \\ \text { VIM } & \text { Virtualized Infrastructure Manager } \\ \text { VL } & \text { Virtual Link } \\ \text { VLAN } & \text { Virtual LAN } \\ \text { VLD } & \text { Virtual Link Descriptor } \\ \text { VLR } & \text { Virtual Link Record } \\ \text { VM } & \text { Virtual Machine } \\ \text { vMANDO } & \text { virtualized Management and Networking Domain } \\ \text { VNF } & \text { Virtualized Network Function } \\ \text { VNFD } & \text { Virtualized Network Function Descriptor } \\ \text { VNFFG } & \text { VNF Forwarding Graph } \\ \text { VNFFGD } & \text { VNF Forwarding Graph Descriptor } \\ \text { VNFFGR } & \text { VNF Forwarding Graph Record } \\ \text { VNFM } & \text { VNF Manager } \\ \text { VNFR } & \text { Virtualized Network Function Record } \\ \text { VNX } & \text { Virtual Networks over Linux } \\ \text { VoD } & \text { Video on Demand } \\ \text { vRGW } & \text { virtual RGW } \\ \text { WAN } & \text { Wide Area Network } \\ \text { WLAN } & \text { Wireless LAN } \\ \text { xDSL } & \text { x Digital Subscriber Line } \\ & \\ \text { VDA }\end{array}$




\section{Capítulo 1}

\section{Introducción}

\subsection{Contexto y Motivación}

De acuerdo con el informe presentado por la Comisión de Banda Ancha [1], la difusión de las Tecnologías de la Información y las Comunicaciones (TICs) y la interconexión global tienen un gran potencial para acelerar el progreso de la humanidad. Así, promover la inversión en conectividad de banda ancha desde una amplia gama de sectores puede ayudar a conseguir el máximo potencial de estas tecnologías y acercar el mundo al objetivo de una sociedad digital inclusiva y accesible para todos. El acceso a Internet desde casa a través de una conexión fija de banda ancha ha representado históricamente la opción más común. El portal Statista ${ }^{1}$ muestra que el número de suscripciones a Internet de banda ancha fija en todo el mundo han aumentado de 220 millones en 2005 a 979 millones en 2017. En este contexto, la red residencial al permitir compartir la conexión de banda ancha que proporciona acceso a Internet entre múltiples dispositivos finales [2], desempeña un rol fundamental en el cumplimiento del objetivo descrito por la Comisión de Banda Ancha.

En la actualidad, la mayoría de tareas que realizamos están vinculadas de una u otra forma con el uso de determinados servicios y aplicaciones que requieren el servicio de conectividad local y hacia Internet que proporciona la red residencial. Por ejemplo, para el teletrabajo, la teleeducación, la atención médica, el entretenimiento, la automatización del hogar, entre otras. Estas aplicaciones o servicios imponen requisitos específicos de conectividad como el ancho de banda, retraso, jitter, disponibilidad, entre otros, lo cual se traduce en la provisión de un determinado nivel de Calidad de Servicio (QoS), con el objetivo de obtener un rendimiento óptimo y un buen nivel de Calidad de Experiencia (QoE). Para abordar esta demanda, desde las Organizaciones de Desarrollo de Estándares (SDOs), como Broadband Forum o la ITU-T, se han propuesto estándares que definen formalmente la arquitectura de la red residencial y modelos de gestión destinados, entre otras cosas, a gestionar el servicio de conectividad para cumplir con los requerimientos específicos de la gran variedad de servicios y aplicaciones que se soportan.

Al analizar los procedimientos de gestión propuestos se identifica claramente el enfoque técnico implementado lo cual representa un problema importante para la gran mayoría de usuarios residenciales que no disponen de conocimientos técnicos y no poseen experiencia alguna en la configuración de redes. Como lo indican Edwards et al. [3] este problema surge porque la red residencial ha adoptado un modelo de gestión al estilo de Internet en el cual se debe garantizar el conocimiento y la experiencia técnica. Esta situación provoca que se restringa la usabilidad o capacidad de gestión de la red residencial, en consecuencia, los usuarios no son capaces de aprovechar todos los beneficios que brinda la red residencial para adoptar nuevos servicios o tecnologías para el hogar que requieren conectividad de red como, por ejemplo, la compartición de videos a través de DLNA (Digital Living Network Alliance ${ }^{2}$ ).

\footnotetext{
${ }^{1}$ https://www.statista.com/statistics/268673/number-of-broadband-internet-subscriptions/

${ }^{2}$ DLNA define directrices de interoperabilidad que permiten compartir medios digitales de manera directa.
} 
Por otra parte, las funciones que incorpora la pasarela o gateway residencial (RGW) determinan las tareas de gestión que se pueden realizar y, por lo tanto, también el grado de usabilidad que tendrá la red residencial. Existen diferentes funciones como el encaminamiento, la Traducción de Direcciones de Red (NAT), el Sistema de Nombres de Dominio (DNS), el Protocolo de Configuración Dinámica de Host (DHCP), el control parental, el filtrado de tráfico, la QoS, entre otros. Para configurar estas funciones el $\mathrm{RGW}$ proporciona una interfaz gráfica de gestión basada en tecnologías web la cual, con frecuencia, está diseñada para entornos de red gestionados por personal especializado. Como se puede deducir, el servicio de conectividad de red que proporciona la red residencial depende en gran medida de las funciones que incorpora el RGW.

En este contexto, el RGW es un dispositivo de red complejo que trata de adaptarse a la evolución de los servicios multimedia que se ejecutan en Internet, aunque su arquitectura cerrada obstaculiza significativamente la innovación. La adición de nuevas funciones al RGW requiere normalmente una actualización de firmware lo cual se traduce en la necesidad de solicitar a los diferentes fabricantes que modifiquen el código para posteriormente ejecutar un proceso de actualización en todos los dispositivos colocados en los hogares de los usuarios. Además, las nuevas funcionalidades pueden experimentar un desempeño no óptimo considerando que el RGW dispone de un hardware con recursos limitados. En este sentido, es primordial que se proponga un nuevo modelo flexible y ágil que permita incorporar nuevas funciones al RGW para mejorar el servicio de conectividad y simplifique los procesos de gestión implícitos.

Para abordar los problemas antes descritos, el estado del arte muestra que existen varias propuestas que aprovechan las últimas tendencias tecnológicas en el ámbito de las telecomunicaciones para definir un nuevo modelo de red para las redes residenciales. Algunos enfoques proponen virtualizar el RGW (vRGW) de tal manera que sus funciones internas sean implementadas como instancias virtuales en la infraestructura en la Nube del ISP y colocando un dispositivo simple de capa 2 (L2) en las instalaciones del usuario. Este modelo está basado en el paradigma de Virtualización de Funciones de Red (NFV) el cual propone que las funciones de red tradicionalmente integradas en hardware especializado puedan ser implementadas en software utilizando tecnologías de virtualización sobre servidores comerciales estándar. Otros enfoques proponen RGWs de próxima generación que aprovechan la programabilidad precisa del plano de datos que introduce el paradigma de las Redes Definidas por Software (SDN). La capacidad de SDN para definir flujos de tráfico detallados y la facilidad para comunicar eventos de red proporciona una flexibilidad extraordinaria para controlar el tráfico de la red residencial. Adicionalmente, al centralizar el plano de control se pueden aprovechar los recursos computacionales del ISP para desarrollar aplicaciones sofisticadas para controlar el plano de datos.

El estado del arte también muestra que existe un claro consenso en cuanto a la propuesta de virtualización del RGW, siendo la arquitectura NERG (Network Enhanced Residential Gateway) de Broadband Forum y la iniciativa CORD (Central Office Re-architected as a Data Center) los ejemplos más sobresalientes. No obstante, es importante tener presente que el paradigma de NFV se centra en proporcionar una réplica virtualizada de un dispositivo de red con hardware específico y al colocar un dispositivo L2 en casa del usuario se restringen las capacidades de direccionamiento necesarias para controlar con precisión flujos de tráfico en la red residencial. Por ejemplo, resulta más eficiente realizar un filtrado de tráfico no deseado localmente antes que realizarlo en las instalaciones del ISP donde se encuentra el vRGW. Adicionalmente, es importante mencionar que estas propuestas mayormente están orientadas a proporcionar las herramientas adecuadas para mejorar la provisión de servicios de telecomunicaciones, de tal manera que el ISP sea capaz de cumplir con las demandas de usuarios de manera ágil y eficiente.

Al momento de proponer un nuevo modelo de red es importante no solamente considerar optimizar los procesos operativos del ISP, sino también abordar el problema de la limitada usabilidad que ofrece el modelo de gestión actual de la red residencial. Para este fin, es necesario considerar el punto de vista del usuario, conocer cuáles son sus necesidades más inmediatas y sobre la base de este conocimiento proponer un nuevo modelo de gestión efectivo. Además, con el objetivo de proponer tal modelo de gestión, es importante implementar un enfoque interdisciplinario que utilice, por ejemplo, técnicas de 
interacción hombre-computadora $\left(\mathrm{HCI}^{3}\right)$ para simplificar los procesos de gestión y adaptarlos a las habilidades y conocimiento de un usuario residencial promedio.

A esto se suma la necesidad de abordar la alta heterogeneidad que presenta el entorno residencial y los requisitos divergentes que presentan los diferentes servicios o aplicaciones que se ejecutan en la red residencial, de acuerdo con el análisis que realizan Grinter et al. [4] y Edwards et al. [3]. Con respecto a la heterogeneidad, es importante considerar que existen diferentes perfiles de usuario, cada uno con preferencias, necesidades y destrezas técnicas específicas. Por ejemplo, usuarios que al trabajar desde casa necesitan que se implemente un trato preferencial al tráfico destinado al teletrabajo, otros que al disponer de un hogar digital necesitan que se garantice la disponibilidad del servicio de conectividad de red y otros que al jugar en línea necesitan que la latencia sea lo mínimo posible para asegurar una excelente QoE. También, que la red residencial es una amalgama de tecnologías, ya sea considerando los dispositivos finales (por ejemplo, PCs de escritorio, dispositivos móviles, consolas de juego, etc) o las tecnologías de red implementadas (por ejemplo, LAN como Fast o Gigabit Ethernet, WLAN como $802.11 \mathrm{~b} / \mathrm{g} / \mathrm{n}$ y WAN como xDSL o FTTH).

Con respecto a los servicios o aplicaciones, como se mencionó previamente, existen diferentes tipos como los de tiempo real sensibles al retraso, no sensibles al retraso, críticos, entre otros. Cada uno de éstos mantiene un conjunto de requerimientos de conectividad local o hacia Internet específicos para proporcionar un rendimiento óptimo. Por ejemplo, los requerimientos del servicio de juego en línea (sensible al retraso) son distintos al servicio de automatización del hogar (crítico).

De esta manera, los problemas que presenta la red residencial se podrían resumir en tres temas puntuales los cuales serán abordados en la presente tesis doctoral:

- Limitada usabilidad de la red debido a la necesidad de disponer conocimientos técnicos.

- Alta heterogeneidad del entorno lo cual dificulta satisfacer todos los requerimientos de los usuarios.

- Limitada flexibilidad para mejorar el servicio de conectividad de red que se proporciona lo cual podría ocasionar que determinados servicios o aplicaciones no obtengan un rendimiento adecuado.

\subsection{Objetivos y Contribuciones}

El principal objetivo de esta tesis doctoral es diseñar una nueva arquitectura de gestión para las redes residenciales con base en los principios de los paradigmas de virtualización de funciones de red y redes definidas por software que permita utilizar el enfoque centrado en el usuario y facilite su implementación para mejorar la usabilidad o capacidad de gestión de la red residencial, abordar el tema de la alta heterogeneidad presente y enriquecer el servicio de conectividad que ésta proporciona de tal manera que se puedan soportar servicios o aplicaciones multimedia avanzadas. Por otra parte, también se considera como objetivo proporcionar un entorno ágil y flexible que le permita al ISP optimizar los procesos de despliegue, gestión y mantenimiento asociados a las redes residenciales a la vez que facilita la creación de nuevos modelos de negocio.

Las propuestas presentadas en esta tesis doctoral no solamente tienen como objetivo abordar los problemas que presenta la red residencial en la actualidad, sino que también permiten que el entorno de gestión concebido evolucione con el tiempo y se adapte a los nuevos requerimientos tanto de los usuarios residenciales como de los servicios o aplicaciones que se ejecutan en la red residencial, así como a nuevas tecnologías emergentes.

Con el fin de cumplir con el objetivo principal, a continuación, se plantea un conjunto de objetivos específicos.

1. Realizar un estudio del estado del arte que permita, en una primera parte, analizar la situación actual de las redes residenciales con el objetivo de reconocer sus limitaciones o problemas desde

\footnotetext{
${ }^{3}$ HCI investiga el diseño y el uso de la tecnología informática, centrada en las interfaces entre personas (usuarios) y computadoras.
} 
la perspectiva del usuario. En una segunda parte, introducir al lector en las tecnologías de Redes Definidas por Software (SDN) y Virtualización de Funciones de Red (NFV) para proporcionarle los fundamentos necesarios que le permitan comprender la contribución de estas tecnologías para mejorar la situación actual de las redes residenciales. En una tercera parte y final, analizar las propuestas relacionadas con el objetivo de identificar aspectos que todavía no han sido resueltos y que constituyen el área de investigación en la cual se centra esta tesis doctoral.

2. Proponer y diseñar una nueva arquitectura de gestión para la red residencial que permita utilizar el enfoque centrado en el usuario y facilite su implementación. La centralidad en el usuario permitirá mejorar la usabilidad de la red al proporcionar un sistema de gestión a medida que considera las necesidades del usuario y que está adaptado a las habilidades de un usuario promedio. Para lograr esta centralidad, el usuario participa activamente en las tareas de gestión de su propia red mediante el uso de diferentes aplicaciones para la Gestión de la Red Residencial, denominadas como "aplicaciones RENEMA". Similar al escenario de las aplicaciones móviles, el usuario escoge las aplicaciones que formarán parte del sistema de gestión con el objetivo de mejorar algún aspecto de la red residencial de acuerdo con sus requerimientos. De esta manera, se abordará también el tema de la alta heterogeneidad del entorno residencial ya que el usuario, al disponer de la flexibilidad para agregar diferentes aplicaciones, es capaz de personalizar su entorno de gestión. Para facilitar la implementación del enfoque centrado en el usuario, la arquitectura proporciona diferentes Servicios de Red Residencial encargados de simplificar la configuración y monitoreo de red de bajo nivel a la cual deben enfrentarse los desarrolladores de aplicaciones RENEMA.

Por otra parte, la arquitectura plantea la definición del Dominio de Gestión y Redes virtualizado (vMANDO) que se encarga de agrupar los componentes SDN y NFV que sustentan la red residencial. A través de este concepto, es posible alcanzar un alto grado de automatización en el despliegue, gestión y mantenimiento de la red residencial. Este aspecto resulta de gran interés para los ISPs ya que les permite optimizar procesos, innovar y ser más competitivos.

La arquitectura de gestión propuesta representa una de las contribuciones de esta tesis doctoral ya que aborda el problema de la usabilidad limitada y el alto grado de heterogeneidad del entorno residencial que se identifican en el estado del arte. Por otra parte, la arquitectura al utilizar SDN y NFV proporciona un entorno ágil y flexible para que el ISP sea capaz de enfrentar los retos asociados a los procesos operativos de las redes residenciales.

3. Proponer un tratamiento de tráfico diferenciado basado en la funcionalidad de Encadenamiento de Función de Servicio (SFC) de NFV que permita proporcionar las condiciones necesarias para satisfacer los requerimientos divergentes de los servicios o aplicaciones que utilizan el servicio de conectividad que proporciona la red residencial con el objetivo de que éstas obtengan un rendimiento óptimo. Este objetivo se conseguirá al implementar un modelo de trabajo colaborativo entre los componentes SDN y NFV que forman parte del Dominio de Gestión y Redes virtualizado (vMANDO) definido en la arquitectura de gestión propuesta. De esta manera, el componente NFV, con base en la solicitud del usuario residencial, se encarga de desplegar una SFC que proporciona un tratamiento específico mientras que el componente SDN se encarga de diferenciar el tráfico de la red residencial que requiere el tratamiento específico para direccionarlo a la SFC correspondiente.

El tratamiento de tráfico diferenciado propuesto representa la segunda contribución de esta tesis doctoral ya que aborda el problema de los requisitos divergentes de los servicios que se ejecutan en la red residencial identificados en el estado del arte.

4. Validar las propuestas presentadas en la tesis doctoral y analizar su factibilidad mediante el planteamiento de un caso de uso y el desarrollo de la correspondiente prueba de concepto. En lo que respecta a la prueba de concepto, se propondrá un escenario de validación compuesto por la infraestructura de computación en la Nube del Proveedor de Servicios de Internet (ISP), diferentes redes residenciales y la red de acceso. En el escenario de validación se realizarán pruebas de desempeño y de provisión de QoS para obtener resultados que permitan verificar la factibilidad de las propuestas presentadas. Por otra parte, se realizará el ejercicio de implementar los diferentes 
componentes de la arquitectura de gestión utilizando el lenguaje de descripción de servicios TOSCA y la plataforma de NFV Tacker.

\subsection{Metodología de Trabajo y Estructura de la Memoria}

La investigación realizada en la presente tesis doctoral es del tipo documental y experimental. En lo que respecta a la parte documental de la investigación, la metodología utilizada consistió en realizar una revisión exhaustiva del estado del arte desde el punto de vista teórico y de propuestas con el objetivo de organizar sistemáticamente el conocimiento adquirido e identificar los problemas existentes en las redes residenciales, así como en las propuestas que tratan de resolverlos. Este proceso permitió definir un conjunto de objetivos específicos para abordar los problemas identificados y que representan las contribuciones de la tesis doctoral. En el proceso de definición de objetivos se ha considerado a SDN y NFV como tecnologías habilitantes y, por lo tanto, se ha requerido de igual manera una profunda comprensión de tales tecnologías.

En lo que respecta a la parte experimental de la investigación, se ha planteado un caso de uso que ha requerido el desarrollo de una prueba de concepto para validar las propuestas presentadas en la tesis doctoral. Para desarrollar la prueba de concepto se han implementado los diferentes bloques funcionales que incluye la arquitectura de gestión propuesta sobre un escenario de validación compuesto por la red residencial, la red de acceso y la infraestructura de computación en la Nube del ISP. En el escenario de validación se han realizado diferentes experimentos con el fin de observar el comportamiento de la arquitectura de gestión y obtener resultados que demuestren la factibilidad de las propuestas.

Es importante mencionar que, considerando los resultados obtenidos de la parte experimental de la investigación, se han identificado temas pendientes por abordar o que necesitan un mayor grado de análisis y que por lo tanto constituyen líneas futuras de investigación.

La memoria de la presente tesis doctoral en la cual se resume el trabajo desarrollado en los últimos años está estructurada en capítulos de la siguiente manera:

- Capítulo 2, Estado Del Arte

En este capítulo se proporcionan las bases teóricas para comprender, por una parte, lo que es una red residencial e identificar los problemas que ésta presenta y, por otra parte, el funcionamiento de SDN y NFV, poniendo énfasis en los conceptos utilizados en el desarrollo de las propuestas. Posteriormente se analizan los trabajos relacionados con el objetivo de identificar problemas que aún no han sido solucionados o aspectos que pueden mejorarse. En la parte final, se proporcionan las conclusiones del capítulo y se detalla un conjunto de requisitos que serán considerados en la propuesta de un nuevo modelo de gestión para la red residencial.

\section{- Capítulo 3, Arquitectura de Gestión Centrada en el Usuario para Redes Residenciales}

En este capítulo se presenta la contribución principal de la tesis doctoral: una arquitectura de gestión para la red residencial con gestión centrada en el usuario. Se definen un conjunto de principios que permiten diseñar la arquitectura y se proporciona una descripción detallada de sus diferentes bloques funcionales y capas. Se introducen nuevos conceptos como la aplicación para la Gestión de la Red Residencial, denominada como "aplicación RENEMA"; los Servicios de Red Residencial, denominados como "RENESEs" y el Dominio de Gestión y Redes virtualizado, denominado como "vMANDO". Adicionalmente, se proporciona información detallada sobre la operación de aplicaciones y servicios para que el lector comprenda de mejor manera el funcionamiento de la arquitectura. En la parte final se realiza un análisis de las propuestas y se proporcionan las conclusiones respectivas.

\section{- Capítulo 4, Tratamiento de Tráfico Diferenciado basado en SDN y NFV}

En este capítulo se presenta la segunda contribución de la tesis doctoral: un tratamiento de tráfico diferenciado para la red residencial con soporte de QoS. Se propone que desde la arquitectura de gestión se coordine el funcionamiento colaborativo entre los componentes SDN y NFV con el 
objetivo de complementar las aportaciones individuales de cada tecnología. De esta manera, se pretende que el componente SDN se encargue de diferenciar el tráfico en la red residencial mientras que el componente NFV se encarga de aplicar un tratamiento específico mediante la asociación de una SFC. Se proporciona una descripción detallada de la estructura interna de vMANDO y se define una aplicación RENEMA encargada de interactuar con las interfaces de la Gestión y Orquestación de NFV para implementar el tratamiento de tráfico diferenciado. En la parte final se realiza un análisis de la propuesta y se proporcionan las conclusiones respectivas.

- Capítulo 5, Validación de las Propuestas

En este capítulo se presenta un caso de uso y se desarrolla una prueba de concepto con el objetivo de verificar la factibilidad de las propuestas presentadas y comprender de mejor manera el funcionamiento de la arquitectura de gestión. Se desarrollan pruebas de desempeño y de provisión de QoS sobre bancos de prueba (testbeds) virtual que tratan de emular un escenario de validación real. Adicionalmente, se detalla el proceso a seguir para implementar vMANDO como un servicio de red utilizando plantillas de despliegue o descriptores. En la parte final se proporcionan las conclusiones respectivas.

\section{- Capítulo 6, Conclusiones y Trabajos Futuros}

En este capítulo se presenta un análisis sobre el cumplimiento de los objetivos propuestos y se proporciona un conjunto de conclusiones generales derivadas del desarrollo de la tesis. Por otra parte, se describe el proceso de divulgación de resultados a través de publicaciones en revistas con factor de impacto y congresos de prestigio, así como la participación en proyectos nacionales. En la parte final se describen aspectos relacionados al ámbito de la tesis que todavía no han sido abordados y que representan líneas futuras de investigación. 


\section{Capítulo 2}

\section{Estado Del Arte}

En los últimos años, las redes han venido experimentando un cambio radical impulsado por el desarrollo de nuevos paradigmas como el de las redes definidas por software (SDN) y la virtualización de funciones de red (NFV). Las promesas de estos paradigmas para fortalecer la innovación y agilizar el desarrollo de nuevos servicios está cambiando la forma en la cual las redes residenciales serán diseñadas, desplegadas, gestionadas y mantenidas en un futuro cercano.

Considerando este hecho, en una primera parte, en este capítulo se analiza la situación actual de las redes residenciales con el objetivo de reconocer sus limitaciones o problemas desde la perspectiva del usuario. Posteriormente, se proporciona una descripción de las tecnologías SDN y NFV para que el lector sea capaz de comprender su utilidad y cómo han sido empleadas en la propuesta de nuevas arquitecturas que permitan mejorar la usabilidad de las redes residenciales en diferentes aspectos, abordar el tema de la heterogeneidad del entorno y enriquecer el servicio de conectividad de red provisto. En la parte final, se analizan los enfoques propuestos por otros autores con el objetivo de identificar aspectos que todavía no han sido resueltos y que constituyen el área de investigación en la cual se enfoca esta tesis doctoral.

\subsection{La Red Residencial}

\subsubsection{Orígenes y Evolución de la Red Residencial}

La red residencial tiene sus orígenes en la combinación de dos hechos fundamentales como lo indican Cummings y Kraut [5]. Por una parte, la creación de ARPANet en 1969 destinada a compartir recursos computacionales entre investigadores del gobierno, industria y academia. Por otra parte, la introducción de los computadores personales por parte de Apple e IBM a principios de los años 80. De esta manera, surge la opción de conectar los computadores personales a redes nacionales como ARPANet ya sea para continuar con el trabajo en casa o para trabajar desde casa en vez de ir a la oficina (teletrabajo). Esta opción solamente estaba al alcance de un determinado grupo de personas, caracterizadas por ser jóvenes o de mediana edad y con estudios superiores. Con el transcurso de los años, el acceso desde casa ha ido evolucionando como consecuencia del advenimiento del mercado masivo de acceso a Internet, vía módems telefónicos en los años 90 y, posteriormente, vía banda ancha a inicios del siglo 21 .

El estudio realizado por Grinter et al. [2], muestra a la red residencial como un conjunto de tecnologías que se convirtieron en una tendencia global debido a la necesidad de compartir el acceso a Internet entre múltiples computadores personales. A parte de este factor, Parekh [6] menciona los siguientes factores que también impulsaron la necesidad de desplegar redes residenciales:

- El alto grado de penetración que experimentaron los dispositivos portátiles como PDAs y teléfonos celulares que proporcionaban a los usuarios servicios de voz y datos. Adicionalmente, estos dispositivos resultaban ser más útiles en casa cuando se disponía de una red con conectividad a Internet a la cual conectarse. 
- El interés de los consumidores por periféricos como los grabadores de vídeo digital y la posibilidad de instalar impresoras y escáneres debido a su reducido coste, originó la necesidad de compartir estos recursos entre múltiples computadores en casa.

- Una nueva era de aparatos inteligentes con capacidad de conexión a Internet, denominado como el Internet de las cosas (IoT), también requieren de la conectividad proporcionada por las redes residenciales.

Por otra parte, el estudio de Grinter et al. [2] también resalta que las personas en adoptar tecnologías de banda ancha se caracterizaban por tener conocimientos de redes, logrando así crear e instalar redes residenciales complejas que comprendían no sólo las tecnologías de infraestructura como hubs, routers, gateways y puntos de acceso inalámbricos, sino también dispositivos orientados a aplicaciones como reproductores de medios y almacenamiento centralizado. Esta afirmación, concuerda con lo dicho por Parekh [6] ya que la compartición de periféricos como impresoras o poner en marcha un dispositivo de IoT requieren, evidentemente, un cierto grado de conocimientos técnicos. Se podría decir entonces que las redes residenciales fueron el resultado de la habilidad para interconectar dispositivos y proporcionarles acceso a Internet que tuvo un determinado sector de la sociedad, caracterizado por tener conocimientos de redes y motivado principalmente por temas laborales.

Ciertamente, la idea de red residencial e Internet impulsaron la evolución de las tecnologías de banda ancha, otorgando la oportunidad de desplegar masivamente redes residenciales en todo el mundo a los proveedores de servicios de Internet. Pero también es importante considerar que las altas velocidades de comunicación han permitido el desarrollo de los denominados servicios multimedia. En la actualidad, este tipo de servicios son utilizados masivamente por la sociedad para realizar diferentes tareas diarias, accediendo a ellos desde diferentes ubicaciones y mediante diferentes terminales, por ejemplo, a través de la laptop ubicada en casa. De esta manera, se podría considerar a la red residencial como un soporte fundamental para el acceso a los servicios multimedia $y$, por ende, al desarrollo de las sociedades.

Al analizar la evolución de las redes residenciales es importante reconocer los siguientes aspectos fundamentales:

- La estructura física o arquitectura de una red residencial no ha cambiado significativamente desde sus orígenes. La pasarela o gateway residencial, denominado como RGW en el contexto de esta tesis doctoral, mantiene las funciones de conectar la red residencial a la red del ISP para alcanzar Internet. Con el transcurso del tiempo, el RGW ha incorporado una interfaz Web gráfica de gestión que permite configurar algunas características del dispositivo como la creación de una red invitada, políticas de calidad de servicio (QoS), filtrado de direcciones MAC, etc.

- Se aprecia una evolución significativa en las velocidades de conexión a Internet proporcionadas por las tecnologías de banda ancha (xDSL y FTTH) al igual que en las velocidades de las tecnologías LAN y WLAN que sustentan la red residencial.

- El contenido al que se accede desde la red residencial ha evolucionado notablemente. Impulsado por el entretenimiento, la interactividad y los dispositivos móviles, en la actualidad el contenido multimedia es el predominante.

- Nuevas tecnologías se han integrado a la red residencial siguiendo la tendencia del hogar inteligente o "smart home". Así, considerando la ubiquidad del uso de teléfonos inteligentes o "smartphones", aparatos domésticos tradicionales han incorporado una conexión WLAN para ser controlados o monitorizados a través de una aplicación que se ejecuta en el smartphone. Similarmente, DLNA o "Digital Living Network Alliance" se ha llegado a convertir en una tecnología común para comunicar diversos dispositivos con el propósito de compartir contenidos en el hogar.

- El modelo de gestión de la red residencial no ha cambiado desde sus inicios. En la actualidad, aún se requieren conocimientos de redes para aprovechar todos los beneficios que la red puede ofrecer más allá del acceso a Internet, lo cual se traduce en la utilización de las herramientas que incorpora el RGW. Estas herramientas, por ejemplo, permiten configurar políticas de QoS o activar el control parental y son específicas de cada fabricante. Además, debido a que están embebidas en el RGW, su rendimiento está restringido por las características del hardware. 
Es importante comprender los orígenes de la red residencial y la evolución que ha tenido para reconocer sus puntos débiles y posteriormente definir una estrategia que permita mejorarlos. Las nuevas tecnologías concebidas para las redes permiten re-definir el paradigma de la red residencial, considerando las verdaderas necesidades del usuario final para asegurar su adaptación a las nuevas tecnologías que se crearán en el futuro.

\subsubsection{Arquitectura de la Red Residencial}

De acuerdo con la ITU-T ${ }^{4}$, una red residencial es la colección de elementos que procesan, administran, transportan y almacenan información, permitiendo así la conexión e integración de múltiples dispositivos de computación, control, monitoreo, comunicación y entretenimiento en el hogar [7]. La Figura 2.1 muestra, de forma simplificada, el conjunto de tecnologías, servicios y aplicaciones potenciales que una red residencial puede sustentar.

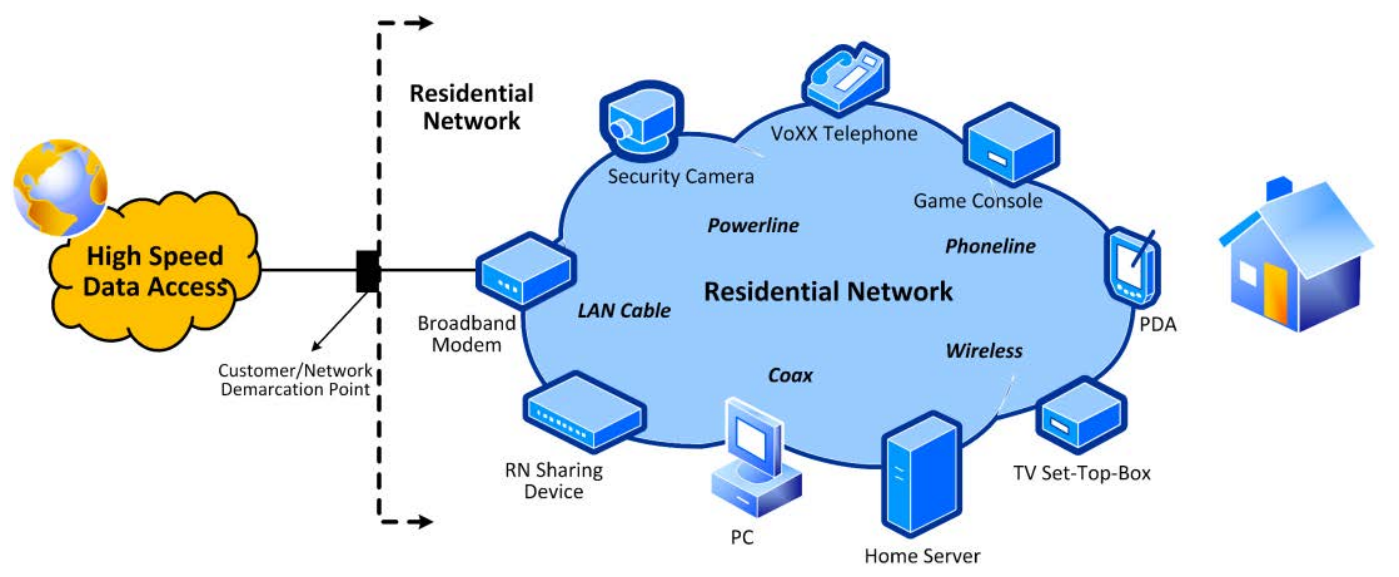

Figura 2.1 Arquitectura simplificada de una red residencial [8]. Un dispositivo de compartición (RN Sharing Device) permite interconectar diferentes dispositivos para formar la red residencial. A su vez, el módem de banda ancha $(\mathrm{BB})$ conecta la red residencial a la red de acceso del proveedor.

Al observar la Figura 2.1 es evidente que, dependiendo del tipo de servicios proporcionados en una red residencial, se pueden obtener estructuras con distintos grados de complejidad. Para abordar esta heterogeneidad, se han elaborado arquitecturas genéricas o de referencia con el objetivo de estandarizar el despliegue de redes residenciales con soporte de servicios multimedia. De esta manera, estas arquitecturas definen diferentes componentes funcionales, así como interfaces de comunicación entre los mismos.

En este contexto, Broadband Forum ${ }^{5}$ propone un Marco para la Entrega de Servicios Múltiples para Redes Residenciales [8] con el objetivo de aprovechar todas las capacidades de los servicios multimedia que ofrece un acceso de banda ancha. Así, los usuarios pueden utilizar múltiples dispositivos y aplicaciones con diferentes requisitos de conectividad (QoS) al mismo tiempo, minimizando el rendimiento deficiente de la aplicación que podría resultar de la contienda por el consumo de recursos. En la Figura 2.2 se muestra la arquitectura propuesta, de la cual se destacan los siguientes componentes funcionales:

- POTS splitter (PS). Considerando el uso de xDSL como tecnología de acceso, este componente permite separar los servicios POTS de los servicios de acceso por banda ancha. Es importante mencionar que en la actualidad el Cable y FTTH también son tecnologías de banda ancha utilizadas frecuentemente.

- Broadband Network Termination (B-NT). Proporciona un punto de terminación de red físico para la señal de la tecnología de acceso de banda ancha y convierte las señales digitales recibidas

\footnotetext{
${ }^{4}$ http://www.itu.int/en/ITU-T/publications/Pages/recs.aspx

${ }^{5} \mathrm{https}: / /$ www.broadband-forum.org/
} 
a un único formato común para la red de distribución local. Un módem xDSL o una ONT (Optical Network Termination) son ejemplos de B-NT. La función de B-NT puede ser integrada con el RG para simplificar la implementación y control de QoS entre la red residencial y el acceso de banda ancha.

- Routing Gateway (RG). Se encarga de interconectar la red residencial con la red de acceso en el B-NT mediante la función de encaminamiento IP que incorpora. El RG juega un rol principal en la gestión de la red residencial proporcionando, por ejemplo, funcionalidades para definir políticas de QoS, gestionar el direccionamiento IP local (DHCP), configurar políticas de seguridad, etc.

- Premises Distribution. Este componente proporciona la conectividad entre el RG y los dispositivos de procesamiento funcional (FPDs). Existen numerosas opciones tecnológicas para el medio de distribución como cable UTP categoría 5, radio frecuencia, cable coaxial, red eléctrica de $\mathrm{AC}$, etc. La arquitectura propuesta asume que la tecnología de distribución utilizada soporta IEEE $802.3 \mathrm{y}$, por lo tanto, la conectividad dentro de la residencia debe ser implementada utilizando tecnologías de conmutación LAN. Por otra parte, la red de distribución local puede requerir la aplicación de técnicas de cableado estructurado para dotar de conectividad a toda la residencia y, en casos donde no se pueda tender cables, se puede recurrir a utilizar puntos de acceso (APs) inalámbricos que implementan, por ejemplo, IEEE 802.11x.

- Functional Processing Device (FPD). Componente con un determinado uso/aplicación que procesa voz, vídeo o datos y que soporta conectividad IP. Ejemplos de FDPs podrían ser Set Top boxes, adaptadores de streaming de audio y adaptadores de teléfonos de VoIP para teléfonos analógicos.

- End User Terminal (EUT). Dispositivo electrónico común que aprovecha indirectamente la conectividad de la red, pero no ha sido específicamente diseñado para hacerlo. Un receptor estéreo es un ejemplo de EUT, ya que aprovecha la conectividad IP de un reproductor MP3 para reproducir la música analógica sin necesidad de estar conectado a la red residencial.

- Functional Processing Device and Terminal (FPD/T). Es un FPD utilizado directamente por el usuario. Ejemplos de FDP/Ts podrían ser computadores, PDAs, impresoras, smartphones, etc.

- Application Service Gateway (ASG). Actúa como un gateway entre la red residencial IP y las redes que no son IP. La red de automatización del hogar es un ejemplo de red no IP. La función de ASG puede ser implementada en el dispositivo físico que implementa la función de RG o en un dispositivo distinto.

- Supplementary Application Network. Ejemplos de este tipo de redes incluyen los sistemas actuales de seguridad en el hogar, control climático y telemetría. Este tipo de redes acceden a las funcionalidades proporcionadas por la red residencial a través del ASG.

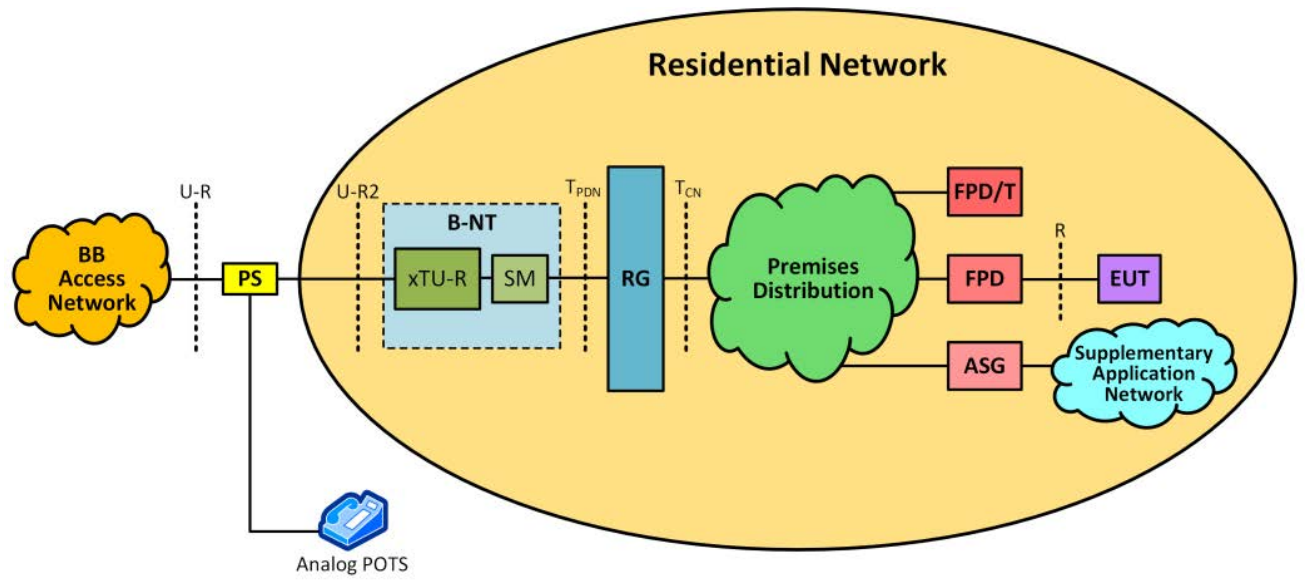

Figura 2.2 Arquitectura funcional para la red residencial propuesta por Broadband Forum [8]. 
- Interfaces. Los componentes funcionales de la arquitectura se comunican entre sí a través de interfaces. Considerando que diferentes componentes pueden ser implementados en un mismo dispositivo físico, algunas interfaces no son apreciables. Las interfaces U-R y U-R2 están especificadas por la tecnología de acceso que conecta la red residencial a la red del ISP. La interfaz $T_{\text {PDN }}$ define la conexión entre el B-NT y el RG, siendo apreciable cuando los componentes no están en el mismo dispositivo físico. La interfaz $\mathrm{T}_{\mathrm{CN}}$ define la conexión entre el RG y la red de distribución local y su especificación depende de la tecnología implementada. La interfaz R es específica del EUT.

Ciertamente, la arquitectura propuesta por Broadband Forum resalta la funcionalidad operacional de la red residencial en la provisión de conectividad externa e interna con soporte de QoS, proporcionando una visión detallada de su estructura física. De esta afirmación se deduce que la red residencial tiene la función de soportar diferentes servicios o aplicaciones que necesitan conectividad de red, es decir, la red residencial proporciona conectividad como servicio a servicios de más alto nivel. Los servicios o aplicaciones soportados son variados e incluyen, por ejemplo, voz, video y sus variantes como el video bajo demanda, entretenimiento como las consolas de video juegos, compartición de archivos digitales, automatización del hogar, etc.

Esta visión es presentada también por la ITU-T en su propuesta de Arquitectura de Red Residencial Genérica con Soporte para Servicios Multimedia [7]. En esta arquitectura, se define a la red residencial mediante la capa de la función de transporte y la capa de la función de aplicación como se muestra en la Figura 2.3. El modelo de transporte describe cómo la red enlaza a los clientes y transfiere los paquetes de datos a través de la red, utilizando para ello los niveles de red, enlace de datos y físico. Por otra parte, el modelo de aplicación describe las características que permiten a los usuarios interactuar con las redes, incluyendo la definición de formatos de datos, el diseño de servicios y el diseño de interfaces. IPTV es un ejemplo de un tipo de servicio.

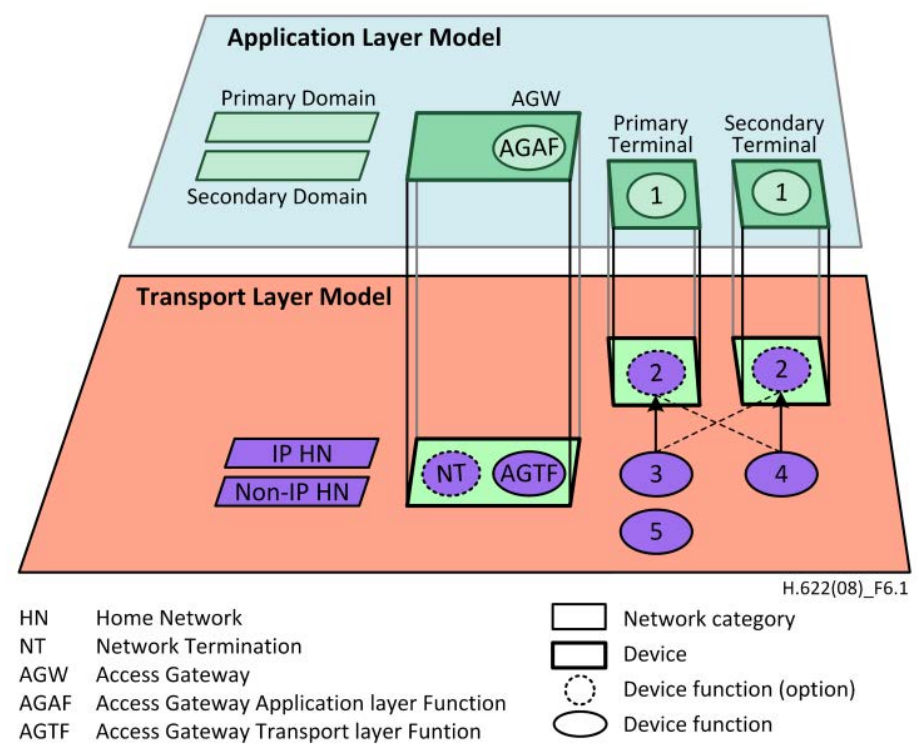

Figura 2.3 Diagrama conceptual del modelo en capas propuesto por ITU-T para la red residencial [7].

A través del modelo de aplicación y la definición de los dominios primario y secundario, la ITU-T resalta la función que desempeña la red residencial en la extensión de la red de acceso y la interconexión de dispositivos. La definición de dominios permite mostrar de una manera distinta la co-existencia de segmentos de red IP y no IP. Además, acentúa la necesidad de establecer características de conectividad como un determinado nivel de QoS para los servicios que pertenecen al dominio primario y la opción que tienen los dispositivos del dominio secundario para acceder a los servicios de conectividad que se ofrecen en el dominio primario. 


\subsubsection{Gestión de la Red Residencial}

La arquitectura propuesta por Broadband Forum define un modelo de gestión para la red residencial. Como se muestra en la Figura 2.4, el modelo determina que tanto la red de acceso como la red residencial son elementos a ser gestionados. En lo que respecta a la red de acceso, el proveedor correspondiente tiene la responsabilidad de gestionarla. No obstante, los resultados de los procesos de gestión pueden ser monitorizados por una tercera parte interesada, por ejemplo, el usuario final.

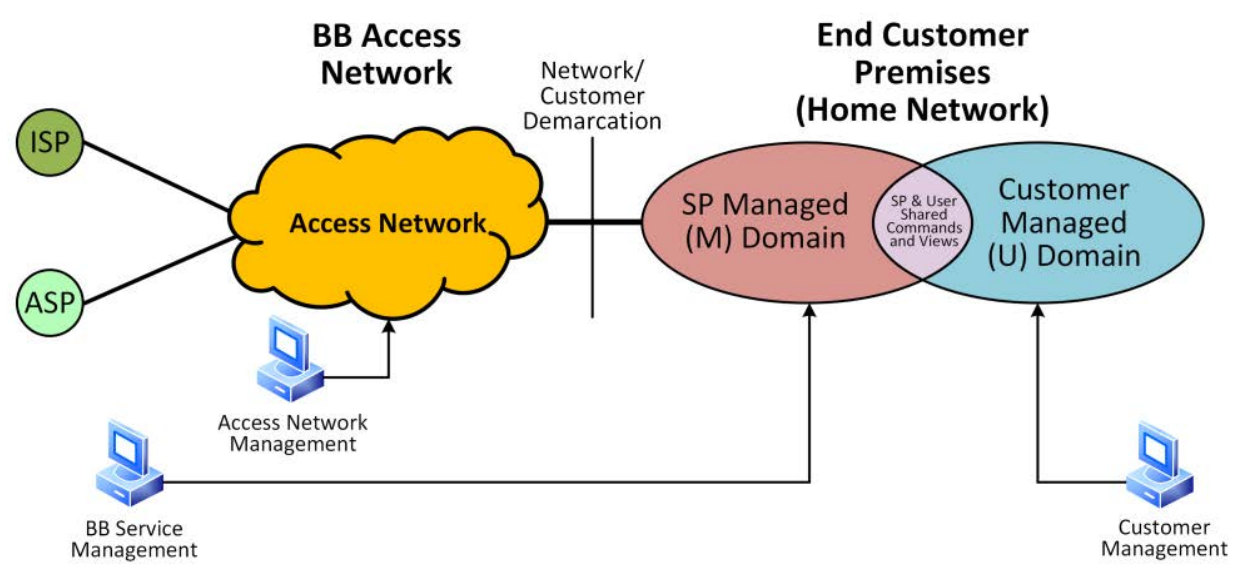

Figura 2.4 Modelo de gestión para la red residencial propuesto por Broadband Forum [8].

En lo que respecta a la red residencial, considerando que su gestión es una tarea compartida entre el proveedor del servicio de banda ancha y el usuario, se definen dos dominios de gestión. En el dominio gestionado por el proveedor (M) se controlan y monitorizan las características del B-NT y del RG remotamente, utilizando protocolos de gestión como NETCONF [9] o CWMP/TR-069 [10]. En el dominio gestionado por el usuario (U) se controlan y monitorizan las características del RG ya sea directamente utilizando la GUI basada en Web que incorpora el propio dispositivo o indirectamente utilizando un cliente software que se ejecuta por separado en un computador.

La definición de dominios realizada por Broadband Forum permite establecer el grado de responsabilidad en cuanto a la gestión de la red residencial. El área superpuesta de la Figura 2.4 indica que existen tareas de gestión que pueden ser realizadas tanto por el proveedor como por el usuario y depende del acuerdo que exista entre ambas partes. Así, el usuario tiene acceso al dominio del proveedor para realizar tareas de control/monitorización predefinidas por el propio proveedor mientras que el proveedor tiene acceso al dominio de usuario para realizar tareas de control/monitorización con base en los requerimientos del usuario.

Las tareas de control/monitorización que se pueden realizar en ambos dominios depende fundamentalmente de las funcionalidades que incorporen tanto el B-NT como el RG con base en las tecnologías WAN/LAN implementadas. En la actualidad, un único dispositivo es capaz de integrar todos los componentes necesarios para desplegar la red residencial. Este dispositivo, denominado como Gateway Residencial (RGW), incorpora principalmente una interfaz WAN para el acceso a los servicios de banda ancha, interfaces Ethernet para la LAN y un Punto de Acceso inalámbrico (AP) para la WLAN. Modelos más avanzados de RGW pueden incluir otras interfaces como POTS o RF. Las funcionalidades a incorporar están estandarizadas por la organización Home Gateway Initiative ${ }^{6}$ (HGI), la cual ha especificado los requerimientos técnicos para una pequeña gama de Gateways de bajo costo y alta capacidad que proporcionan soporte de comunicación multiservicio para los entornos residenciales y SOHO (Small Office Home Office) [11].

La especificación realizada por HGI incluye una arquitectura de gestión para la red residencial basada en el estándar TR-069 propuesto por Broadband Forum. En esta arquitectura, la gestión del RGW es, en

\footnotetext{
${ }^{6} \mathrm{http}: / /$ www.homegatewayinitiative.org/
} 
gran parte, realizada por el proveedor de servicios de forma remota, mediante un Servidor de auto configuración (ACS) que se incorpora en un Sistema de Gestión Remota (RMS). Por otra parte, se establece que el usuario puede tener una interfaz para ajustar, de forma limitada, la configuración del RGW. Dicha interfaz pertenece a la aplicación de gestión local y está basada en tecnologías Web. La arquitectura de gestión está enfocada en realizar las siguientes funciones principales:

- Gestión del dispositivo

- Gestión de QoS

- Gestión de la seguridad

- Gestión de la configuración

- Gestión de actualizaciones de firmware

- Monitoreo del desempeño

- Diagnóstico y resolución de problemas

Al analizar las funciones de la arquitectura de gestión propuesta por HGI, se podría decir que existe una correspondencia con el Marco de Gestión para la Interconexión de Sistemas Abiertos (OSI) para aplicaciones CCITT, denominado como X.700 [12]. Este marco proporciona una base común para el desarrollo coordinado de las normas de gestión, definiendo las áreas funcionales para la gestión de fallos, configuración, contabilidad, desempeño y seguridad (FCAPS). Considerando este hecho, se podría decir que la gestión de la red residencial no representa una actividad trivial en lo absoluto. Edwards et al. [3] mencionan que la red residencial ha adoptado un modelo de gestión al estilo de Internet que requiere un cierto nivel de conocimientos de redes y, por lo tanto, no es adecuado para usuarios que no son expertos en tecnología. De esta manera, la afirmación de Broadband Forum al indicar que la gestión de la red residencial es una actividad compartida entre proveedores y usuarios es válida siempre y cuando se cumpla el requerimiento de conocimientos técnicos por parte del usuario.

Por otra parte, la arquitectura propuesta por HGI al asignar la mayor parte de responsabilidad en la gestión de la red al proveedor, lo obliga a enfrentarse a la heterogeneidad que los entornos residenciales presentan, tal y como lo describen Grinter et al. [4] y Edwards et al. [3]. De esta manera, el proveedor debe definir un modelo de gestión que satisfaga, en gran medida, los problemas más comunes presentes en las redes residenciales debido a lo complejo y costoso que representa satisfacer cada requisito de un usuario en particular. Desde el punto de vista del usuario, esta situación podría causar un malestar al percibir que sus necesidades no son atendidas a pesar de estar pagando por tales servicios.

\subsubsection{Perspectiva del Usuario Residencial}

Como se analizó en la sección 2.1.2, la red residencial puede ser considerada como un contexto de uso donde la conectividad es un recurso a ser controlado. El control de tal recurso no representa una tarea trivial como se analizó en la sección 2.1.3 y, por tal motivo, se ha definido un marco de gestión para la red residencial. El marco propuesto restringe la intervención del usuario residencial en las tareas de gestión de su propia red, lo cual representa una decisión errónea que desmejora la usabilidad de la red residencial. Así, un marco de gestión debería definirse considerando el punto de vista del usuario, sus necesidades más inmediatas. Es así que, considerando la heterogeneidad resultante de los diferentes perfiles de usuario (necesidades y preferencias), así como los diferentes servicios o aplicaciones que se utilizan en casa, los problemas más comunes a los cuales los usuarios residenciales podrían enfrentarse son clasificados en cuatro aspectos principales de mejora como se detalla a continuación.

- Facilidad de Gestión. Shehan y Edwards [13] mencionan que al principio de la era de las redes el concepto de facilidad de uso no fue considerado por las primeras arquitecturas ya que la experiencia técnica estaba garantizada. La red residencial heredó esta necesidad de conocimientos técnicos para realizar tareas relacionadas con la gestión y el mantenimiento. Como consecuencia, la gestión de la red residencial sigue siendo un misterio para la mayoría de los usuarios.

Un enfoque de "hoja en blanco" permitiría la creación de un nuevo paradigma para las redes residenciales, en el cual, los usuarios jugarían un papel primordial en su gestión. Para cumplir con este requisito, es necesario desarrollar nuevas herramientas de gestión basadas en el conocimiento y experiencia de los usuarios residenciales. Para la configuración de la red residencial, dichas 
herramientas deben implementar un enfoque "fácil de usar", lo cual permitiría eliminar los inconvenientes y barreras encontrados en la actualidad.

- Visibilidad. En general, los usuarios residenciales tienen información muy limitada sobre el estado de sus redes. Esta afirmación se sustenta en el trabajo realizado por Chetty et al. [14], el cual menciona que preguntas como "¿Por qué mi conexión a Internet es tan lenta?" suelen presentarse cuando una página web tarda mucho tiempo en cargarse $\mathrm{y}$, que la respuesta más común es afirmar que el ISP no está proporcionando el ancho de banda apropiado. Ejemplos de este tipo afectan negativamente a la Calidad de Experiencia percibida (QoE). Por lo tanto, es necesario mantener informado al usuario residencial sobre lo que está sucediendo en su red: qué dispositivos de red están conectados, cuál es el consumo de ancho de banda de cada uno de ellos, cuáles son las páginas web visitadas o los archivos descargados, etc. Además, la información proporcionada sobre el estado de la red debe ser significativa para los usuarios residenciales, evitando detalles técnicos como direcciones IP o puertos TCP que probablemente no podrán ser interpretados correctamente.

La mejora de la visibilidad no sólo sirve como medio para educar y capacitar a los usuarios sobre los servicios de banda ancha en general, sino que también cambia el paradigma sobre la relevancia de saber lo que está sucediendo con nuestras propias redes.

- QoS/QoE. Para hacer frente a la diversidad de dispositivos conectados a las redes residenciales y al tráfico multimedia generado o utilizado, Liu et al. [15] argumentan que el enfoque tradicional de "mejor esfuerzo" utilizado por las redes IP debe complementarse con mecanismos que garanticen un nivel adecuado de QoS. Hoy en día, el RGW suele incluir algunos mecanismos para configurar políticas de QoS, los cuales están basados en las recomendaciones realizadas por HGI [16]. Sin embargo, el uso de tales mecanismos requiere un cierto nivel de habilidades técnicas que está fuera del alcance de la mayoría de los usuarios.

Un enfoque centrado en el usuario podría ser considerado con el objetivo de definir un entorno en el cual no se requieran habilidades técnicas y los usuarios residenciales sean agnósticos de la infraestructura de IT existente. Por ejemplo, si un usuario residencial percibe una resolución de vídeo deficiente, la "visibilidad" de la red le proporcionaría información sobre el consumo de recursos de la red y la "facilidad de gestión" le mostraría cómo mejorar la QoE por medio de las herramientas de gestión existentes. Más allá de este enfoque manual, la red sería lo suficientemente inteligente como para realizar una adaptación automática basada en las actividades de los usuarios residenciales.

- Seguridad. Las tecnologías inalámbricas se utilizan ampliamente en las redes residenciales debido a su comodidad y flexibilidad. Sin embargo, dado que el medio de transmisión está a disposición de cualquier persona, pueden presentarse graves riesgos de seguridad. En algunos casos, se puede obtener fácilmente el acceso a una red inalámbrica mal protegida simplemente descargando y ejecutando una herramienta de hacking. Para evitar estos problemas de seguridad, Stimpson et al. [17] mencionan que existen múltiples recomendaciones de seguridad que pueden implementarse en modernos RGWs, como cambiar las contraseñas predeterminadas, usar algoritmos modernos de autenticación y cifrado o configurar filtros de direcciones MAC. Además, el RGW proporciona maneras de saber quién está conectado a la red, pero típicamente en términos de las direcciones MAC de los dispositivos conectados, lo cual no se adapta a las habilidades del usuario.

Gross y Rosson [18] explican en su trabajo que la seguridad es un tema que preocupa a los usuarios, pero normalmente no saben cómo proteger sus datos confidenciales o cómo usar las herramientas de software de seguridad desarrolladas para ese propósito. De esta manera, existe la oportunidad de desarrollar nuevos mecanismos para mejorar la seguridad de la red residencial. Por ejemplo, agregando un mecanismo de acceso adicional controlado por el usuario que permita la conectividad de red solamente a dispositivos conocidos.

El análisis previo de estos cuatro aspectos de mejora permite concluir que, en general, los usuarios residenciales no son capaces de gestionar sus propias redes. No sólo porque no cuentan con los 
conocimientos técnicos necesarios, sino también porque no cuentan con un modelo de gestión adaptado a sus necesidades, preferencias y destrezas técnicas. De esta manera, se requiere un rediseño del modelo de gestión de la red residencial considerando un enfoque centrado en el usuario que permita a los usuarios tomar el control de sus propias redes para mejorar la usabilidad y abordar el tema de la alta heterogeneidad del entorno. Por otra parte, un nuevo modelo de gestión también permitiría enriquecer el servicio de conectividad que proporciona la red residencial y no depender por completo de las funcionalidades que un fabricante en particular incorpora a un modelo determinado de RGW.

\subsection{Conceptos Generales sobre Redes Definidas por Software}

\subsubsection{Definición y Arquitectura}

Como lo mencionan Nunes et al. [19], aunque el concepto de Redes Definidas por Software (Softwaredefined Networking, SDN) es reciente, las ideas sobre "redes programables" y "desacoplar la lógica de control" no son nuevas. De hecho, existieron varios esfuerzos en el campo de las redes programables que son considerados precursores del paradigma SDN actual y que establecieron las bases para varias ideas que actualmente se observan. Todas las propuestas tenían un objetivo en común: facilitar la evolución de la red.

En el trabajo realizado por Feamster et al. [20], se muestra la evolución del concepto de redes programables hasta llegar al paradigma SDN a través de tres etapas fundamentales:

- Las redes activas (desde mediados de los años 90 hasta principios de los 2000) introdujeron funciones programables en la red, promoviendo una mayor innovación.

- La separación del plano de datos y de control (a partir de 2001 a 2007) permitió desarrollar interfaces abiertas entre estos dos planos.

- OpenFlow y los sistemas operativos de red (desde 2007 hasta 2010) representaron la primera adopción generalizada de una interfaz abierta, desarrollando maneras de hacer escalable y práctica la separación entre control y datos.

Considerando que OpenFlow había obtenido un gran impulso, diferentes operadores de red y empresas tecnológicas crearon la Open Networking Foundation $\left(\mathrm{ONF}^{7}\right)$ con el objetivo de promover SDN y estandarizar el protocolo OpenFlow. Con el transcurso del tiempo y, debido al alta expectativa entorno a este nuevo paradigma, varias organizaciones de estandarización, como la ITU-T, IETF, IRTF, etc., se sumaron para facilitar una transición sin problemas de la tecnología de red tradicional a los nuevos protocolos y arquitecturas como SDN.

De acuerdo con la ONF, "SDN es una arquitectura de red emergente donde la función de control de red está desacoplada de la función de envio de paquetes, siendo esta última directamente programable. La migración del control, el cual ha estado fuertemente unido a los dispositivos de red individuales, hacia dispositivos informáticos accesibles, permite abstraer la infraestructura subyacente a aplicaciones y servicios de red, los cuales pueden tratar a la red como una entidad lógica o virtual" [21].

La ONF afirma que las soluciones basadas en SDN permiten hacer frente a la naturaleza dinámica de las aplicaciones de hoy en día, adaptar la red a las necesidades empresariales cambiantes y reducir significativamente la complejidad de operaciones y gestión. A continuación, se listan los beneficios que una arquitectura basada en SDN proporcionaría:

- Control centralizado de entornos multi proveedor

- Reducción de la complejidad a través de la automatización

- Mayor tasa de innovación

- Mayor fiabilidad y seguridad de la red

- Control de red más granular

- Mejor experiencia del usuario

\footnotetext{
${ }^{7}$ https://www.opennetworking.org/
} 
Con respecto a la arquitectura SDN, la ONF ha desarrollado una recomendación, denominada como TR502 [22], donde se detallan los distintos componentes arquitectónicos de referencia. En la recomendación se indica que el objetivo de SDN es proporcionar interfaces abiertas que permitan el desarrollo de software que pueda controlar la conectividad proporcionada por un conjunto de recursos de red y el flujo de tráfico de red a través de ellos, junto con la posible inspección y modificación del tráfico que se puede realizar en la red. Estas funciones primitivas se pueden abstraer en servicios de red arbitrarios.

La Figura 2.5 muestra las interfaces y componentes de la arquitectura SDN, los cuales se explican a continuación:

- La capa de infraestructura (plano de datos) comprende un conjunto de uno o más elementos de red, cada uno de los cuales contiene un conjunto de recursos de procesamiento de tráfico o de envío de tráfico. Los recursos son siempre abstracciones de entidades o capacidades físicas subyacentes. Los elementos de red exponen sus capacidades hacia la capa de control (plano del controlador) a través de interfaces Southbound o D-CPI.

- La capa de control (plano del controlador) comprende un conjunto de uno o más controladores SDN, cada uno de los cuales tiene control exclusivo sobre un conjunto de recursos expuestos por uno o más elementos de red. El controlador SDN traduce los requisitos de las aplicaciones y ejerce un control de bajo nivel sobre los elementos de red, proporcionando a la vez información relevante a las aplicaciones SDN.

- La capa de aplicación (plano de aplicación) comprende una o más aplicaciones, cada una de las cuales tiene control exclusivo de un conjunto de recursos expuestos por uno o más controladores SDN. Las aplicaciones SDN comunican sus requerimientos de red hacia la capa de control (plano) a través de las interfaces Northbound o A-CPI, llamadas frecuentemente NBIs.

- La gestión comprende todas las funciones específicas que pueden ser realizadas por los Sistemas de Soporte a las Operaciones (OSS) en los diferentes planos. Por ejemplo, la configuración inicial de los elementos de red en el plano de datos, monitorizar el rendimiento del sistema en el plano del controlador o configurar los acuerdos de nivel de servicio (SLAs) en el plano de aplicación. Adicionalmente, en todos los planos se configuran las asociaciones de seguridad para que las funciones distribuidas se comuniquen de forma segura.

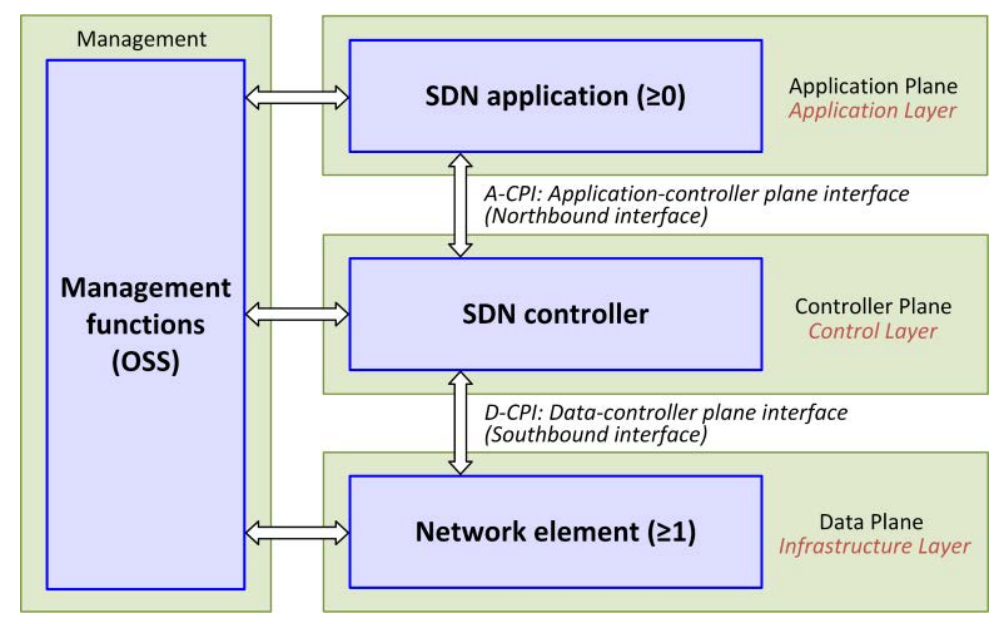

Figura 2.5 Arquitectura SDN [22]. El concepto de plano de datos en el contexto de la arquitectura SDN incluye funciones de procesamiento y envío de tráfico. El plano de datos puede incluir el subconjunto mínimo necesario de funciones de control y gestión.

\subsubsection{Funcionamiento de SDN}

El funcionamiento de SDN está determinado por la interacción entre los componentes que pertenecen a las diferentes capas de la arquitectura a través de las interfaces Northbound y Southbound. De esta 
manera, se hará uso del protocolo OpenFlow en la interfaz Southbound para comprender de mejor manera el funcionamiento de SDN. Shukla [23] define a OpenFlow como un protocolo abierto basado en estándares, el cual establece cómo el plano de datos puede ser configurado y controlado por un controlador externo. Como se muestra en la Figura 2.6, un elemento de red contiene una o más tablas de flujo. Una tabla de flujo está formada por entradas de flujo, cada una de las cuales determina cómo los paquetes que pertenecen a un flujo serán procesados y enviados a través de la red. Las tablas de flujo reemplazan a todas las demás tablas de envío que son utilizadas en dispositivos de red tradicionales. Mediante el uso de OpenFlow, el controlador puede crear y gestionar las tablas de flujo, al igual que mantener una visión completa de la red que controla.

Nunes et al. [19] indican que las entradas de flujo en OpenFlow consisten típicamente en: (1) Reglas de coincidencia, utilizadas para verificar la coincidencia de los paquetes entrantes. Los campos de coincidencia pueden contener información encontrada en el encabezado del paquete, el puerto de ingreso y los metadatos. (2) Contadores, utilizados para recopilar estadísticas de un flujo en particular, tales como el número de paquetes recibidos, el número de bytes y la duración de la entrada de flujo. (3) Un conjunto de instrucciones o acciones que se aplicarán a los paquetes coincidentes.

La Figura 2.7 resume el funcionamiento de SDN. Cuando un paquete llega a un dispositivo SDN con soporte para OpenFlow, los campos del encabezado del paquete se extraen y se comparan con los campos de coincidencia de las entradas de flujo, comenzando en la tabla 0. Si se encuentra una coincidencia, los contadores asociados se actualizan y se aplica el conjunto de instrucciones asociadas con la entrada de flujo. Si no existe coincidencia alguna, la acción tomada por el dispositivo dependerá de las instrucciones definidas por la entrada de flujo "table-miss", por ejemplo, soltar el paquete, continuar el proceso de comparación en la siguiente tabla de flujo o reenviar el paquete al controlador a través de la interfaz Southbound.

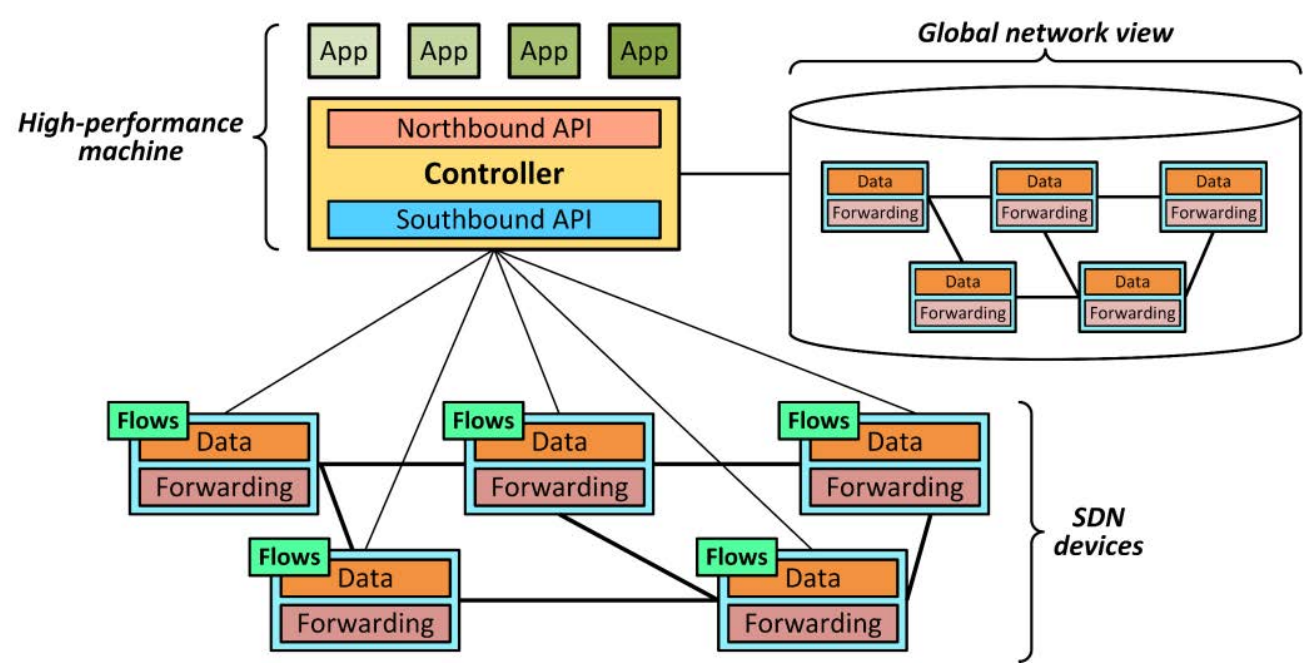

Figura 2.6 Vista general del funcionamiento de SDN [24].

Cuando un paquete (Packet-In) es reenviado al controlador, éste lo entrega a las aplicaciones que han solicitado recibirlo. Las aplicaciones procesan el paquete con el objetivo de decidir qué hacer con el tráfico al cual pertenece el paquete; dicha decisión se expresa mediante una entrada de flujo. Adicionalmente, se debe elegir la operación a realizar con la entrada de flujo. En la parte derecha de la Figura 2.7 se muestran las operaciones disponibles. Como paso final, la tabla de flujos se actualiza con base en el procesamiento realizado.

Es importante distinguir la forma en la cual se realizan operaciones con las entradas de flujo. Por una parte, la aplicación puede realizar operaciones de forma reactiva en respuesta a la información que la red le proporciona. El controlador informa a la aplicación acerca de los eventos que se producen en la red. Los eventos pueden referirse a un paquete individual que ha sido recibido por el controlador (Packet-In) o algún cambio de estado en la topología de red, por ejemplo, un nuevo dispositivo que se 
conecta. Por otra parte, la aplicación puede realizar operaciones de forma proactiva, las cuales no requieren la notificación explícita de un evento de red por parte del controlador.

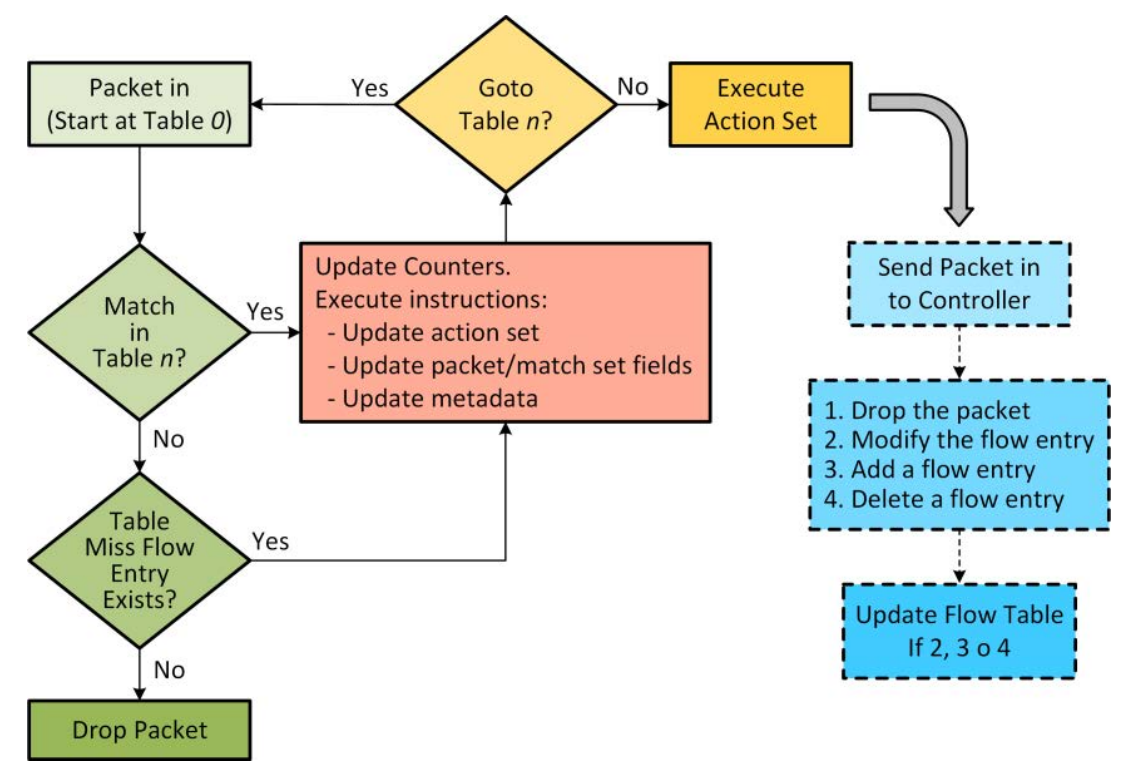

Figura 2.7 Diagrama de flujo del procesamiento de paquetes que realiza un dispositivo OpenFlow [25], [23].

\subsubsection{El Controlador SDN e Interfaces}

Como se ha mencionado anteriormente, SDN permite abstraer los recursos de red subyacentes a aplicaciones y servicios de red. Para lograr este objetivo, el controlador debe mantener una estructura o arquitectura en particular. Göransson et al. [24] presentan una arquitectura genérica para el controlador SDN que se muestra en la Figura 2.8. En esta arquitectura se destacan las interfaces o APIs (Aplication Programming Interfaces) Southbound y Northbound, módulos base que proporcionan servicios específicos y aplicaciones SDN que frecuentemente se incluyen con el controlador.

En la sección anterior se indicó que el API Southbound es utilizado para interactuar con los dispositivos SDN, siendo OpenFlow uno de los protocolos más utilizados. No obstante, existen otras propuestas que permiten cumplir algunos de los objetivos de SDN. Estas propuestas utilizan de forma remota las funciones que se incorporan en los dispositivos de red, empleando métodos tradicionales como SNMP, CLI, NETCONF o APIs REST (Representational State Transfer). Ciertamente, estas propuestas alternativas pueden ser consideradas interfaces Southbound ya que permiten manipular los dispositivos de red y proporcionar la inteligencia y comportamiento previsible que se espera en una red controlada por SDN. Sin embargo, este tipo de soluciones están destinadas a actuar sobre la configuración del dispositivo y los protocolos de red que éste soporta. Por lo tanto, estas soluciones no permiten ejercer un control detallado del tráfico, como lo hace OpenFlow, ya que no permiten definir reglas de envío en el plano de datos.

Continuando con la descripción de la anatomía del controlador SDN, los módulos base se encargan de presentar una visión abstracta de la infraestructura de red subyacente a las aplicaciones externas a través de servicios. Estos servicios son accesibles a través del API Northbound. Las aplicaciones consumen estos servicios para proporcionar nuevas funcionalidades de control. El controlador puede definir un diseño basado en plugins para implementar los servicios base con el objetivo de adaptarse a los requisitos de una red individual.

Para las aplicaciones SDN, una función clave proporcionada por el controlador es el API que permite acceder a la configuración de la red. En algunos casos, este API Northbound es una interfaz de bajo nivel que proporciona acceso a los dispositivos de red de una manera común y consistente. En este caso, esa aplicación es consciente de los dispositivos individuales, pero está protegida de sus diferencias. En otros casos, el controlador puede proporcionar un API de alto nivel que proporciona una abstracción de 
la propia red, de modo que el desarrollador de la aplicación no debe preocuparse de los dispositivos individuales, sino de la red como un todo. La arquitectura de referencia SDN propuesta por la ONF [22] también describe la opción para implementar la recursividad en la capa de aplicación. Las aplicaciones pueden ofrecer servicios a través del API Northbound para simplificar y fomentar la creación de nuevos servicios avanzados o funcionalidades con un mayor nivel de abstracción. Por ejemplo, una aplicación de control de acceso a la red (NAC) podría utilizar el servicio de conmutación de capa 2 (L2) subyacente para implementar su funcionalidad sin necesidad de implementar todo el servicio de conmutación de L2. Del mismo modo, la aplicación NAC también podría ofrecer su funcionalidad como un servicio a otras aplicaciones.

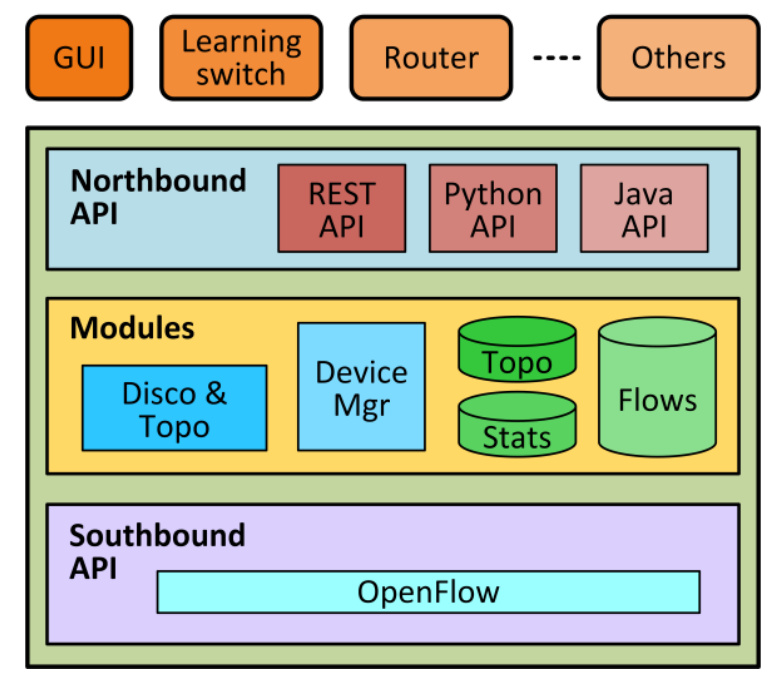

Figura 2.8 Arquitectura genérica de un controlador SDN [24].

Como se puede notar, el API Northbound desempeña un rol crítico en la creación de herramientas para gestionar la red y, por lo tanto, en la consolidación del paradigma SDN. Sin embargo, la falta de estandarización ha obligado a que diferentes controladores propongan diferentes tipos de APIs. Aunque existe un consenso sobre el concepto de servicios básicos y recursividad entre los diferentes controladores, el API Northbound es altamente heterogéneo debido a que cada controlador SDN trata de imponer su propia visión acerca del modelo operativo de este API. Esta situación promueve el efecto "lock-in" del controlador lo cual representa una desventaja significativa que pone en peligro el principio de apertura SDN.

Como lo mencionan Trois et al. [26], el API Northbound puede ser implementado por un controlador de diferentes maneras: como un marco que proporciona un API, como un lenguaje de programación SDN de alto nivel o como un API remoto. Las dos primeras opciones representan un modelo dependiente del controlador, caracterizado por atar a los desarrolladores a una tecnología de controlador específica y restringir la portabilidad de las aplicaciones. Por otra parte, al estar los planos de aplicación y control fuertemente acoplados, ambos deben ejecutarse en el mismo servidor y deben implementarse con el mismo lenguaje de programación.

Con el fin de superar la dependencia de la plataforma del controlador SDN, diferentes controladores también proporcionan un API Northbound basado en REST, el cual se ha convertido en el API remoto predominante. Debido a que cada controlador tiene su propia visión acerca de la implementación del API basado en REST, se han presentado diferentes propuestas para guiar su correcta implementación, tal es el caso del grupo de trabajo NBI de la ONF [27], el Software-Defined Networking Research Group (SDNRG) [28] y Li et al. [29]. Por definición, la tecnología REST es extensible y proporciona un acoplamiento débil entre clientes y servidores, sin embargo, no es adecuado para todos los casos de uso. Las aplicaciones externas podrían necesitar un canal de comunicación bidireccional y full-duplex para interactuar con los servicios base $\mathrm{u}$ otras aplicaciones. HTTP es un protocolo unidireccional donde las funciones de los servidores y los clientes no son intercambiables y una solicitud siempre se inicia por 
un cliente. HTTP también utiliza un paradigma petición-respuesta que restringe que los clientes y los servidores puedan comunicarse entre sí simultáneamente. En cuanto a las aplicaciones en tiempo real, la naturaleza "locuaz" de HTTP y la iniciación/terminación continua de conexiones aumenta significativamente el tiempo necesario para procesar el encabezado y la carga útil para miles de pares de solicitud/respuesta. Además, los servicios proporcionados por el API están restringidos por el número reducido de verbos HTTP estándar, lo cual podría perjudicar la expresividad del API.

En la actualidad, el grupo de trabajo NBI de la ONF ha propuesto un enfoque distinto para el API Northbound. Como se muestra en la Figura 2.9, en lugar de definir otra API más, este grupo de trabajo ha definido un API en la parte superior de las APIs base del controlador, con un nivel de abstracción mayor. De esta manera, las capacidades de la interfaz se basan en "propósitos" o "intents" en lugar de la programación detallada de las tablas de OpenFlow. Este enfoque permite separar completamente las implementaciones de sistemas consumidores y proveedores y, representar solicitudes humano/máquina originadas en el consumidor, tan simple como sea posible [30].

Por ejemplo, un API basada en intents permitiría a las aplicaciones realizar solicitudes abstractas como "permitir que el host A se comunique con el host B". En respuesta a esta solicitud, el controlador SDN realizaría la programación automática de flujos en todos los dispositivos que forman parte de la trayectoria desde el host A al B. En comparación con un API tradicional, la obtención del mismo resultado involucraría realizar la programación manual de todos los dispositivos que forman parte del trayecto entre el host A y B, lo cual incrementaría la complejidad de la aplicación. De acuerdo con Görasson et al. [24], el concepto de intents se basa en tres características fundamentales:

- Abstracción: El objetivo de un controlador SDN, al igual que con los sistemas operativos en general, es ocultar los detalles del hardware subyacente a la aplicación que se ejecuta en la parte superior.

- Declarativo: Especificar qué hacer, en lugar de como hacerlo, es una característica de los sistemas declarativos.

- Agnóstico del protocolo: Una interfaz declarativa abstracta oculta detalles del proceso de programación de red, permitiendo que diferentes protocolos se utilicen en diferentes situaciones.

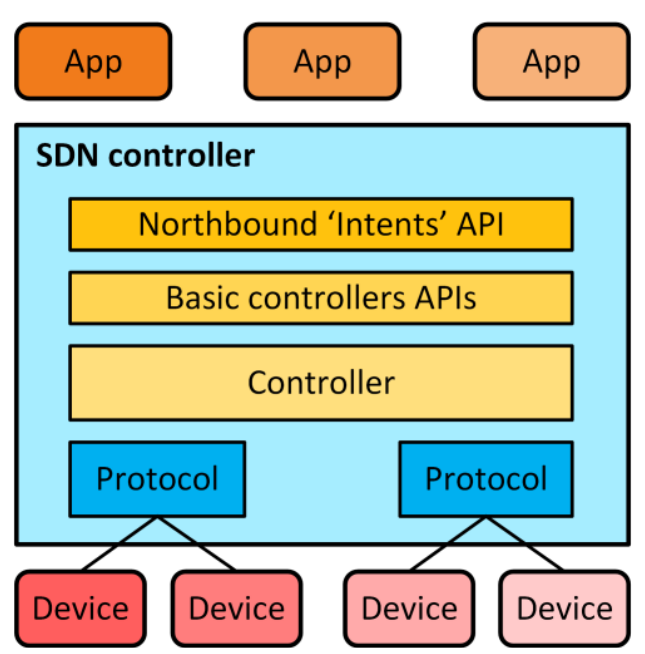

Figura 2.9 Interfaz Northbound basada en "propósitos" o "intents". Representa un paso hacia la creación de un API que elimina la necesidad de conocer y comprender los detalles de los dispositivos en la red física [24].

\subsubsection{Revisión de los Controladores SDN existentes}

Ciertamente, SDN representa un cambio de paradigma trascendental en las redes caracterizado por impulsar la innovación como nunca se ha visto antes. Esta situación, ha motivado a que distintas partes interesadas implementen una gran variedad de controladores SDN con el objetivo de promover y fortalecer este nuevo paradigma. La mayoría de controladores actuales se basan en la estructura genérica mostrada en la Figura 2.8. Sin embargo, cada controlador implementa una arquitectura con 
características específicas considerando el dominio de aplicación. Diferentes autores han realizado estudios exhaustivos con el propósito de clasificar a los controladores SDN existentes considerando determinados aspectos funcionales y no funcionales. Por ejemplo, Chayapathi et al. [31] hacen una distinción entre controladores SDN de código abierto como OpenDayLight ${ }^{8}$ o Floodlight $^{9}$ y, controladores comerciales como VMware $\mathrm{NSX}^{10}$.

Kreutz et al. [32], con base en la clasificación de controladores que realizan, indican que la mayoría de controladores son centralizados y multiproceso. Adicionalmente, destacan que la API Northbound es muy diversa y que los modelos de consistencia y tolerancia a fallos sólo están presentes en controladores específicos como ONOS ${ }^{11}$. Con respecto a la interfaz Northbound, argumentan que la interoperabilidad es un problema a abordar considerando que éste fue el primer problema que intentaron resolver las interfaces Southbound (OpenFlow).

Salman et al. [33] realizan un estudio comparativo entre controladores SDN considerando aspectos como la compatibilidad entre plataformas, soporte de interfaces Southbound y Northbound, soporte de OpenFlow, la alianza de organizaciones para desarrollar controladores, entre otros. De su estudio concluyen que ONOS y OpenDaylight son los controladores más destacados. Estos controladores presentan una asociación de proveedores de redes y comunidades de investigación muy conocidas y tienen un plan de desarrollo claro. Con respecto a ONOS, destacan que su arquitectura está diseñada para mantener redes a gran escala o redes de operadores al igual que proporciona soporte para redes híbridas. Con respecto a OpenDayLight, destacan su capacidad para soportar diferentes tipos de aplicaciones, siendo el primer controlador en ingresar al dominio de IoT. Adicionalmente destacan la amplia gama de interfaces Southbound que soporta y la compatibilidad con OpenStack Neutron ${ }^{12}$.

Cox et al. [34] destacan la importancia de la interfaz East-west para determinar la forma en que los controladores interactúan entre sí para compartir información $\mathrm{y}$, por lo tanto, representa una característica adicional a considerar con el objetivo de evitar eventuales problemas de interoperabilidad entre controladores SDN. Además, esta interfaz se puede usar para mejorar la comunicación entre dominios e inter-dominios y, mejorar la escalabilidad y la interoperabilidad de las implementaciones de SDN. Los autores también indican que, aunque existen interfaces East-west para algunos controladores como Onix, HyperFlow, DIFANE, entre otros, sus comunicaciones son generalmente privadas e ilegibles entre controladores heterogéneos.

Considerando que OpenDayLight y ONOS son dos proyectos predominantes en el desarrollo de SDN, Bondkovskii et al. [35] realizan una evaluación comparativa de la interfaz Northbound entre estos controladores utilizando la configuración de "traffic-mirroring" como caso de uso. Han definido y utilizado criterios de evaluación simples, con base en lo fácil que es: examinar la red, agregar una nueva función de red, cambiar una función existente y eliminar una función. Concluyen que estos controladores se encuentran en constante desarrollo y si bien se han realizado avances importantes se mantienen deficiencias en las interfaces Northbound, particularmente con respecto a la facilidad de uso, documentación y totalidad de la funcionalidad.

De la revisión del estado del arte de controladores, se observa que OpenDayLight y ONOS ciertamente se han convertido en referentes dentro del ecosistema SDN. No obstante, esta situación no significa que el resto de controladores no contribuyan al fortalecimiento del paradigma SDN. Dependiendo del caso de uso o aplicación, se dispone de una gran variedad de opciones que deben ser analizadas para escoger la opción que mejor se adapte.

\footnotetext{
${ }^{8} \mathrm{https}: / /$ www.opendaylight.org/

${ }^{9} \mathrm{https}$ ://floodlight.atlassian.net/wiki/spaces/floodlightcontroller/overview

${ }^{10} \mathrm{https}: / /$ www.vmware.com/products/nsx.html

${ }^{11} \mathrm{http}: / /$ onosproject.org/

${ }^{12} \mathrm{https}: / /$ docs.openstack.org/security-guide/networking/architecture.html
} 


\subsection{Conceptos Generales sobre Virtualización de Funciones de Red}

\subsubsection{Definición y Marco Arquitectónico}

Es innegable que el paradigma SDN ha cambiado el rumbo de las redes para lograr la tan esperada evolución de la red. No obstante, existen otros paradigmas como la Virtualización de Funciones de Red o NFV que también están contribuyendo a sentar las bases de las redes modernas. NFV se origina de la necesidad de suplir las deficiencias en las redes tradicionales, las cuales están caracterizadas por estar centradas en los dispositivos de red. Chayapathi et al. [31] indican que los dispositivos de red tradicionales están fabricados para ejecutar funciones específicas y las redes de datos creadas están adaptadas y personalizadas para cumplir criterios de eficiencia de manera efectiva. Por otra parte, el software que se ejecuta en el hardware con diseño personalizado está fuertemente acoplado a éste y enfocado exclusivamente en realizar las funciones específicas del dispositivo. Esta situación ocasiona que las redes tradicionales presenten las siguientes limitaciones:

- Flexibilidad limitada

- Restricciones de escalabilidad

- Desafíos en cuanto al plazo de lanzamiento de nuevos productos

- Problemas en la capacidad de uso o gestionabilidad

- Altos costos operacionales y de inversión

- Consideraciones de migración

- Sobre-aprovisionamiento de capacidad

- Interoperabilidad

Como lo relatan Mijumbi et al. [36] en su estudio, en respuesta a esta situación, los proveedores de servicios de telecomunicaciones más representativos del mundo escribieron conjuntamente una documentación técnica o "white paper" denominado como "Network Functions Virtualisation: An Introduction, Benefits, Enablers, Challenges \& Call for Action" [37]. Posterior a la publicación del documento técnico, el Instituto Europeo de Normas para las Telecomunicaciones (ETSI) fue seleccionado como sede para el Grupo de Especificación de la Industria para NFV (ETSI ISG NFV ${ }^{13}$ ), habiendo publicado hasta la fecha más de 60 recomendaciones para el ecosistema NFV.

De acuerdo con el documento técnico, NFV tiene como objetivo transformar la forma en que los operadores de red diseñan las redes a través de la evolución de la tecnología de virtualización de TI estándar para consolidar varios tipos de equipos de red en servidores, conmutadores y almacenamiento de gran volumen estándar de la industria, los cuales podrían ser ubicados en Centros de Datos, Nodos de Red y en la localidad del usuario final. Esto implica la implementación de funciones de red en software que pueda ejecutarse en una variedad de servidores estándar de la industria. Además, estas funciones de red pueden ser movidas o instanciadas en varias ubicaciones de la red según sea necesario, sin necesidad de instalar nuevos equipos.

En la Figura 2.10 se representa la propuesta que plantea NFV. Se observa que el software está desacoplado del hardware, lo cual ofrece la posibilidad de utilizar cualquier hardware comercial disponible para implementar el "sabor virtualizado" de funciones de red muy específicas. La virtualización de las redes abre nuevas posibilidades en cuanto a su implementación y gestión. De acuerdo con el criterio de Stallings [38], si el paradigma NFV se implementa de forma efectiva y eficiente, se pueden proporcionar varios beneficios en comparación con los enfoques de las redes tradicionales. A continuación, se resumen los beneficios potenciales:

- Reducción de CapEx (Capital Expenditure) y OpEx (Operational Expenditure) mediante la utilización de servidores y conmutadores comerciales básicos que permiten consolidar los equipos y explotar las economías de escala. Se puede aplicar el enfoque "pay-as-you grow" para eliminar el exceso de aprovisionamiento a la vez que se reducen los costos de gestión de la red.

- Capacidad para innovar y desplegar servicios rápidamente, lo cual permite reducir el tiempo de lanzamiento de nuevos servicios que se adapten a los cambios en los requerimientos de la

\footnotetext{
${ }^{13} \mathrm{http}: / /$ www.etsi.org/technologies-clusters/technologies/nfv
} 
empresa, aprovechar las nuevas oportunidades de mercado y mejorar el retorno de la inversión de nuevos servicios.

- Facilidad de interoperabilidad debido a interfaces estandarizadas y abiertas.

- Agilidad y flexibilidad para satisfacer las demandas cambiantes a través de la rápida ampliación o reducción de los recursos que soportan los servicios.

- Posibilidad de introducir servicios orientados a objetivos geográficos o conjuntos de clientes.

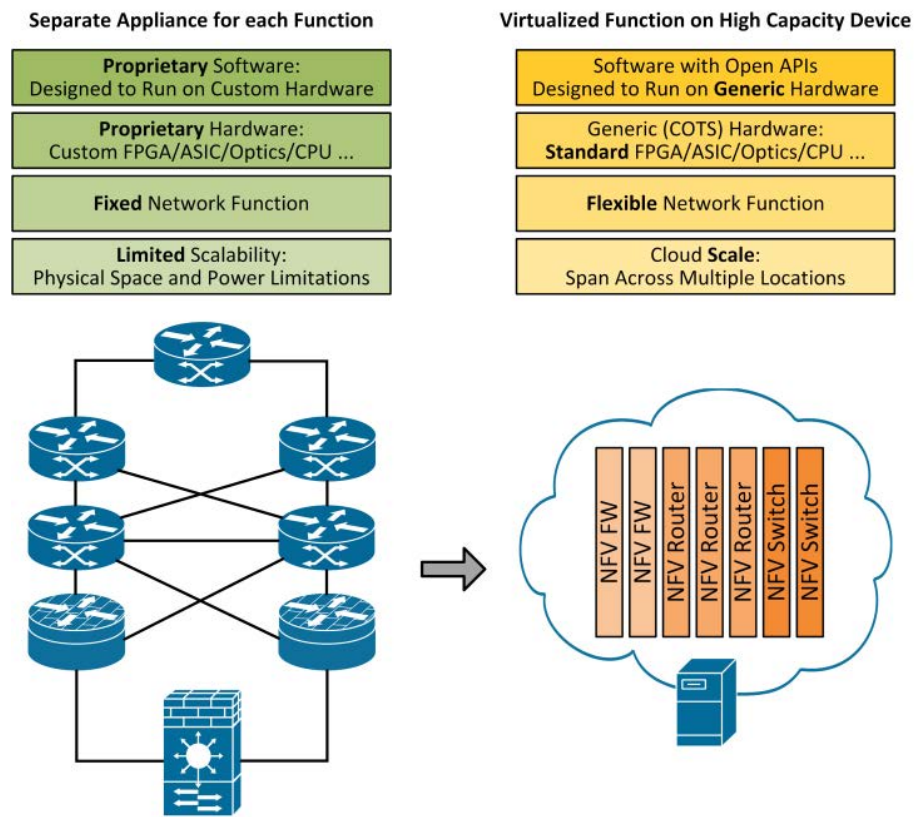

Figura 2.10 Paradigma NFV. Con este nuevo paradigma, las funciones de red pueden implementarse en cualquier hardware genérico que ofrezca los recursos básicos para procesamiento, almacenamiento y transmisión de datos [31].

En el contexto de NFV, la implementación virtual de una función de red se conoce como Función de Red Virtualizada (VNF). Se pretende que una VNF realice una determinada función de red, por ejemplo, enrutador, conmutador, firewall, balanceador de carga, etc. Considerando que las VNFs pueden ser provistas por diferentes proveedores y los operadores de red pueden elegir una combinación de proveedores y funciones que mejor se adapte a sus necesidades, se necesita definir un marco de estandarización. De esta manera, ETSI ha desarrollado un documento que describe el marco arquitectónico funcional, la filosofía de diseño de las funciones de red virtualizadas y la infraestructura de apoyo y cómo los elementos necesarios para realizar NFV se pueden implementar de una manera estandarizada para permitir la interoperabilidad [39]. En la Figura 2.11 se muestra el marco arquitectónico de referencia para NFV en el cual se reconocen los siguientes bloques funcionales y los principales puntos de referencia o interfaces:

- Función de red virtualizada (VNF). Representa la implementación en software de una función de red tradicional, capaz de ejecutarse en una infraestructura de virtualización genérica y no propietaria.

- Elemento de gestión (EM). Ejecuta la típica funcionalidad de administración para una o varias VNFs.

- Infraestructura de NFV (NFVI). Representa la totalidad de todos los componentes de hardware y software que crean el entorno en el que se despliegan, gestionan y ejecutan las VNFs. NFVI puede abarcar varias ubicaciones (distribuido), siendo la red que proporciona conectividad entre estas ubicaciones parte de la Infraestructura de NFV. Desde la perspectiva de VNF, la capa de virtualización y los recursos de hardware se presentan como una sola entidad que proporciona a la VNF los recursos virtualizados deseados. 
- Gestión y Orquestación (MANO) de NFV. El desacoplamiento de una VNF de los recursos de hardware subyacentes presenta nuevos desafíos de gestión como la instanciación de VNFs en ubicaciones apropiadas para cumplir con el servicio deseado, asignación y escalamiento de recursos de hardware para las VNFs, seguimiento de instancias, etc. De esta manera, se define MANO para hacer frente a estos desafíos de gestión. Como se observa en la Figura 2.11, MANO está compuesto por diferentes bloques funcionales, cada uno de ellos con funciones específicas que interactúan con la infraestructura de NFVI, VNFs y OSS/BSS a través de puntos de referencia bien definidos. Más adelante se proporcionará una descripción más detallada de MANO, así como de sus bloques funcionales.

- Descripción de la infraestructura, VNF y servicio. Como se indicará más adelante en detalle, NFV utiliza plantillas de despliegue o descriptores para describir los atributos y requisitos necesarios para desplegar un servicio de red y sus respectivos componentes sobre la NFVI. Existen diferentes tipos de descriptores los cuales son utilizados internamente por NFV-MANO para automatizar el proceso de despliegue.

- Sistemas de Soporte a las Operaciones/Negocio (OSS/BSS). Representan el conjunto de operaciones del operador y funciones de soporte de negocios que no son explícitamente capturadas en el marco arquitectónico de NFV, pero se espera que tengan intercambios de información con bloques funcionales de NFV-MANO. Es importante mencionar que el componente OSS/BBS existente puede agregar funciones adicionales si no están soportadas por una cierta implementación de NFV-MANO.

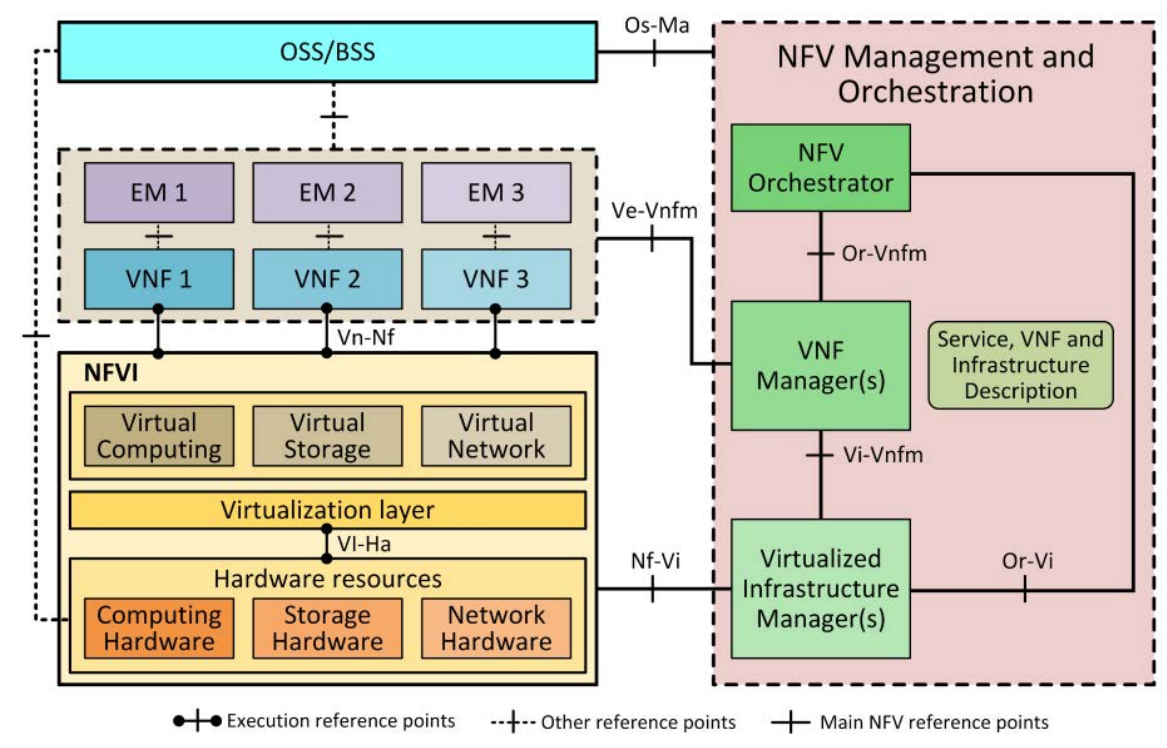

Figura 2.11 Marco arquitectónico de referencia para NFV [39].

- Puntos de referencia. Constituyen interfaces entre bloques funcionales. Los puntos de referencia principales y de ejecución se muestran con líneas continuas y pertenecen al campo de acción de $\mathrm{NFV}$, los cuales representan objetivos potenciales para la estandarización. Los puntos de referencia de línea discontinua están disponibles en implementaciones actuales, pero pueden necesitar extensiones para manejar la virtualización de funciones de red. En la actualidad, estos puntos de referencia están fuera del campo de acción de NFV. En la sección de Gestión y Orquestación de NFV se analizarán estos puntos de referencia con más detalle.

\subsubsection{Servicios de Red}

NFV proporciona las herramientas necesarias para controlar el ciclo de vida de los servicios de red de forma ágil, flexible y dinámica. En este contexto, ETSI argumenta que un Servicio de Red (NS) se puede ver arquitectónicamente como un grafo de envío de funciones de red (NFs) interconectadas a través de 
una infraestructura de red de soporte. Por otra parte, OASIS ${ }^{14}$ (Organization for the Advancement of Structured Information Standards) complementa la definición al indicar que un NS es una composición de funciones de red que define una especificación funcional y de comportamiento de extremo a extremo [40]. El NS incluye dos tipos de gráficos:

a. Un grafo de Topología de Conectividad de Red (NCT) que especifica las funciones de red virtualizadas (VNFs) que componen el servicio y las conexiones lógicas modeladas como enlaces virtuales (VLs) que necesitan ser creadas dinámicamente sobre la infraestructura física.

b. Uno o más grafos de envío de VNF (VNFFGs) que especifican cómo se envían los paquetes entre VNFs a través del grafo de topología de conectividad de red para lograr el comportamiento de servicio de red deseado. El VNFFG a su vez contiene rutas de envío de red (NFPs) que describen un flujo de tráfico en el NS basado en políticas de decisión.

Un VNFFG también es conocido como una Cadena de Función de Servicio (SFC), término proporcionado por el IETF [41]. En el presente documento los términos SFC o VNFFG son utilizados de forma intercambiable para hacer referencia al mismo concepto.

La topología de conectividad de red especifica la conexión de las diferentes VNFs y el flujo de datos a través de esas conexiones, independientemente de los elementos de red físicos subyacentes. Por el contrario, el grafo de envío de VNFs define la secuencia de VNFs a ser recorridas por un conjunto de paquetes que coinciden con determinados criterios, por ejemplo, todos los paquetes destinados al puerto TCP 1547 del host con IP 2.3.4.1. La Figura 2.12 muestra un ejemplo de un gráfico NCT correspondiente a un servicio de red que implementa tres diferentes VNFs. Cada VNF incorpora puntos de conexión (CPs) para conectarse a los diferentes enlaces virtuales. Adicionalmente, CP01 y CP02 representan los puntos finales del propio servicio de red. Con el fin de proporcionar una determinada funcionalidad, diferentes VNFFGs o SFCs pueden ser definidos sobre el mismo NCT.

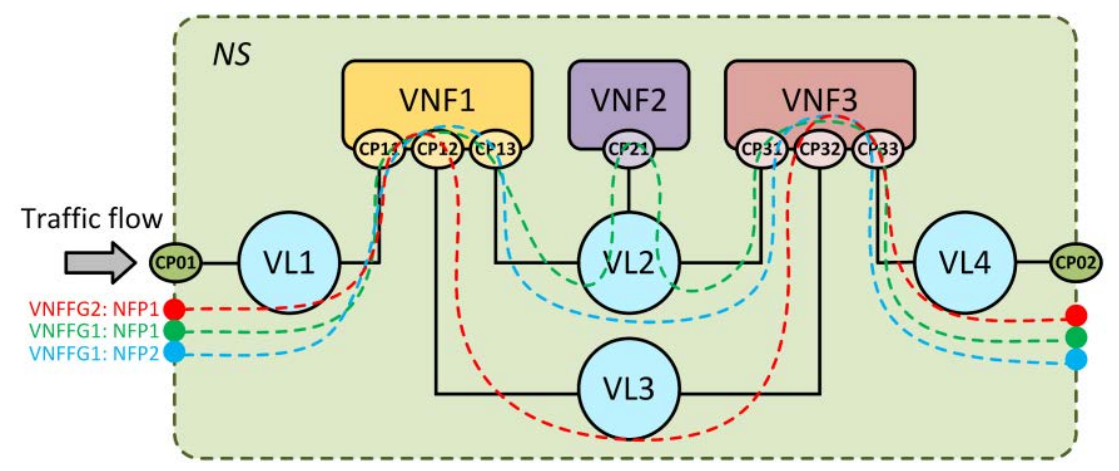

Figura 2.12 Diferentes grafos de envío de VNF pueden ser definidos sobre el mismo grafo de topología de conectividad de red [42].

\subsubsection{Gestión y Orquestación de NFV (NFV-MANO)}

La implementación y operación de servicios en NFV requiere de herramientas basadas en software para la gestión y orquestación de funciones de red virtualizadas. En este sentido, ETSI ha propuesto un marco para la Gestión y Orquestación (MANO) de NFV [42] a cargo del aprovisionamiento de funciones de red virtualizadas (VNFs) y la ejecución de operaciones relacionadas, como la configuración de VNFs y la infraestructura en la que funcionan. Stallings [38] indica que, aparte de las tareas de gestión y orquestación del entorno NFV que de por sí ya son complejas, la funcionalidad de MANO adquiere una complejidad adicional debido a la necesidad de interoperar y cooperar con los OSS/BSS existentes. Así, MANO proporciona funcionalidades de gestión a clientes cuyo entorno de red consiste en una mezcla de elementos físicos y virtuales.

La Figura 2.13 muestra la estructura funcional de NFV-MANO y sus puntos de referencia fundamentales sin especificar implementación alguna. Los puntos de referencia son de gran importancia ya que

\footnotetext{
${ }^{14} \mathrm{https} / / /$ www.oasis-open.org/
} 
representan posibles objetivos de desarrollo en proyectos de código abierto y/o posterior estandarización, garantizando de esta manera la interoperabilidad y portabilidad de servicios de red y las VNFs constituyentes. Cada uno de los bloques funcionales tiene un conjunto bien definido de responsabilidades y opera en entidades bien definidas, utilizando la gestión y la orquestación según corresponda dentro del bloque funcional, así como aprovechando los servicios ofrecidos por otros bloques funcionales. A continuación, se proporciona una descripción más detallada de los componentes de MANO con el objetivo de complementar la información proporcionada en la sección 2.3.1.

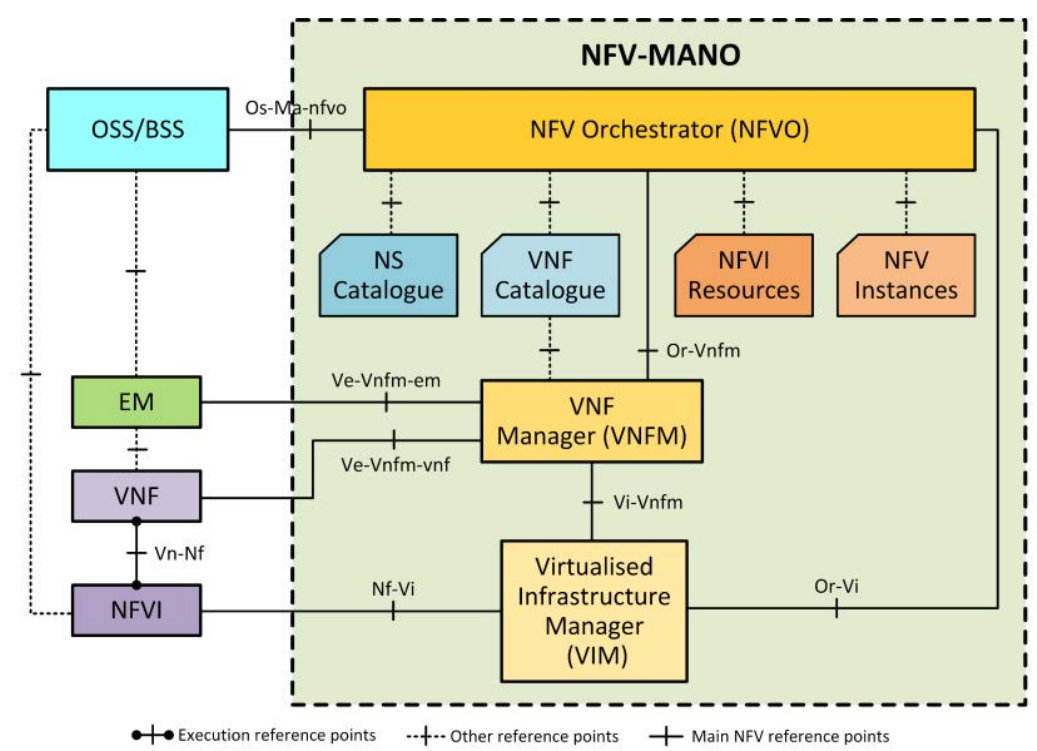

Figura 2.13 Marco arquitectónico de NFV-MANO con puntos de referencia [42].

\section{- Orquestador de NFV (NFVO)}

Este componente es responsable de la orquestación de servicios de red y la orquestación de recursos de NFVI a través de múltiples VIMs. En lo que respecta a la orquestación de servicios de red (NSs), el NFVO es responsable de la gestión del ciclo de vida del servicio de red, para lo cual realiza las siguientes operaciones fundamentales:

- Gestión de plantillas de despliegue de NS y paquetes de VNF (incorporación de nuevos NSs y paquetes de VNF).

- Instanciación del NS y gestión del ciclo de vida de la instancia (actualización, consulta, escalado, medición de rendimiento, recopilación de eventos y correlación, terminación).

- Gestión de la instanciación de gestores de VNF (VNFMs), siempre que sea necesario.

- Gestión de la instanciación de VNFs en coordinación con los VNFMs.

- Gestión de la topología de las instancias del NS (crear, actualizar, consultar o eliminar VNFFGs).

- Gestión y evaluación de políticas para las instancias del NS y VNF (políticas relacionadas al escalamiento, recuperación de fallos, rendimiento, etc.).

En lo que respecta a la orquestación de recursos, NFVO coordina, autoriza, libera y emplea recursos NFVI que pueden estar o no distribuidos en diferentes localidades. Esto lo hace mediante la participación con los VIM directamente a través de sus APIs Northbound en lugar de relacionarse directamente con los recursos NFVI.

\section{- Gestor de VNF (VNFM)}

El VNFM es responsable de la gestión del ciclo de vida de VNF (instanciación, actualización, consulta, escalado, modificación, terminación). Un VNFM puede servir a una sola VNF o múltiples. Entre las funciones que realiza un VNFM se encuentran las siguientes:

- Instanciación de VNF y su configuración, la cual está especificada en la plantilla de despliegue correspondiente. 
- Verificación de la factibilidad de instanciación de VNF, si es necesario.

- Actualización/mejora del software de la instancia de VNF.

- Escalamiento out/in y up/down de la instancia de VNF.

- Rol de coordinación y adaptación general para la configuración y la presentación de informes de eventos entre el VIM y el componente Gestión de Elementos (EM).

\section{- Gestor de infraestructura virtualizada (VIM)}

El VIM es responsable de controlar y gestionar los recursos de computación, almacenamiento y red de la infraestructura de NFV. Este componente proporciona interfaces Northbound que soportan la gestión de los recursos virtualizados que ofrece un determinado NFVI. A continuación, se listan las principales funciones que realiza el VIM:

- Orquestar la asignación, actualización, liberación y recuperación de los recursos de NFVI, así como gestionar la asociación de los recursos virtualizados con los recursos físicos correspondientes.

- Soportar la gestión de grafos de envío de VNF (crear, consultar, actualizar, eliminar).

- Gestionar en un repositorio la información relacionada con el inventario de recursos de hardware NFVI y recursos de software.

- Gestionar las imágenes de software (agregar, eliminar, actualizar, consultar, copiar) según lo solicitado por otros bloques funcionales NFV-MANO.

- Recopilar información de rendimiento y fallos de recursos de hardware, software y virtualizados.

- Gestionar catálogos de recursos virtualizados que se pueden consumir desde el NFVI.

\section{- Repositorios NFV-MANO}

Asociados con NFVO hay cuatro repositorios de información necesarios para las funciones de gestión y orquestación:

- El catálogo de servicios de red contiene los servicios de red utilizables, los cuales están descritos en una plantilla de despliegue en términos de VNFs, enlaces virtuales que las conectan y especificaciones de funcionamiento y despliegue.

- El catálogo de VNF contiene los paquetes de VNF que han sido incorporados a la plataforma de NFV. Un paquete está compuesto por la plantilla de despliegue o descriptor, imágenes de software, políticas de elasticidad, entre otras. Un paquete presenta la imagen completa de la VNF, combinando los parámetros de despliegue y operativos (desde el descriptor), la imagen que se va a utilizar con ella y la configuración con la que debe desplegarse.

- El repositorio de instancias de NFV mantiene información de las instancias de NS y VNF en ejecución. Esta información es representada mediante registros y reflejan cualquier cambio en el estado de funcionamiento de servicios de red y VNFs.

- El repositorio de recursos de NFVI contiene información sobre los recursos que están disponibles, reservados y asignados. Esta información es proporcionada por cada VIM considerando que este bloque funcional interactúa directamente con la infraestructura.

\subsubsection{Elementos de Información de MANO}

En la sección anterior se indicó que MANO define un conjunto de repositorios para realizar el despliegue y posterior gestión de las instancias de NS y VNF. En este contexto, Garay et al. [43] indican que los procesos de despliegue de servicios y de gestión global del ciclo de vida correspondiente se basan en los elementos de información que describen el servicio de red y sus componentes, tanto como plantillas en un catálogo de servicios y como registros de instancias. La Figura 2.14 (a) muestra los elementos de información definidos por ETSI. Es importante mencionar que un servicio de red puede contener funciones de red físicas (PNF) y que un grafo de envío de VNF (VNFFG) puede hacer referencia a otros elementos de información en el servicio de red, como PNF, VL y VNF. Adicionalmente, un VNFFG también contiene un elemento denominado como ruta de envío de red (NFP) que describe un flujo de tráfico en el NS basado en políticas de decisión. 


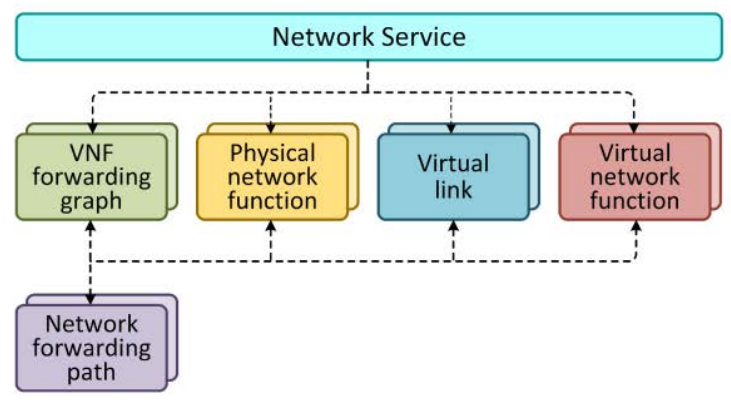

(a)

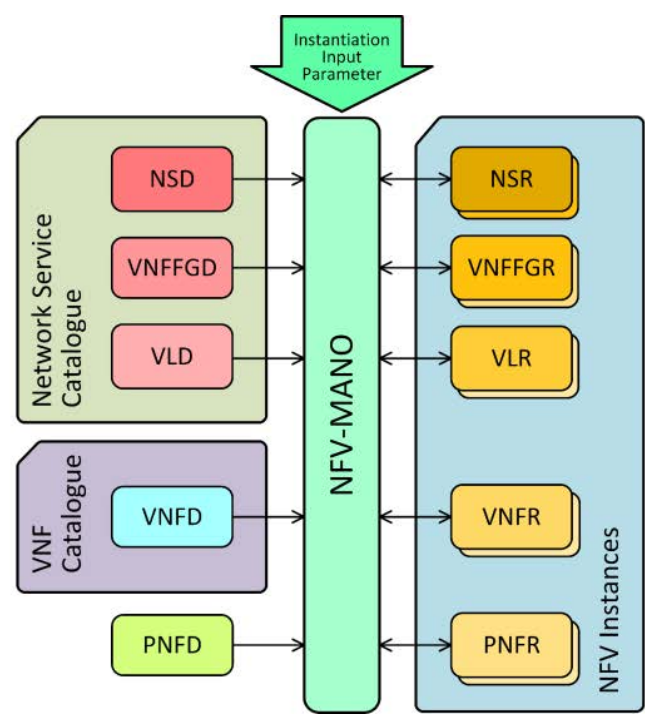

(b)

Figura 2.14 Elementos de información: (a) Estructura de alto nivel, (b) Distribución en catálogos y registros [42].

A continuación, se listan las plantillas de despliegue definidas en NFV para describir por completo los atributos y requisitos necesarios para la puesta en marcha del servicio de red.

a. Los Descriptores de Servicio de Red (NSDs) se consideran elementos de información de servicio de red de nivel superior y permiten especificar el servicio de red a ser creado. NSD hace referencia a todos los demás descriptores que describen componentes que forman parte del servicio de red.

b. Un descriptor de VNF (VNFD) describe la VNF en términos de su despliegue y requisitos de comportamiento operacional. VNFD es principalmente utilizada por el gestor de VNFs (VNFM) en el proceso de instanciación de la VNF y de la gestión del ciclo de vida de la correspondiente instancia.

c. Un descriptor de grafo de envío de VNF (VNFFGD) describe la topología (o una parte) del servicio de red, haciendo referencia a VNFs, PNFs y enlaces virtuales que los conectan.

d. Un descriptor de enlace virtual (VLD) describe los requerimientos de recursos necesarios para el enlace entre VNF, PNF y puntos finales del servicio de red, basado en las opciones de enlace que están disponibles en el NFVI.

e. Un descriptor de función de red física (PNFD) describe los requisitos de conectividad, interfaz e indicadores clave de rendimiento (KPIs) de enlaces virtuales para una función de red física adjunta.

El NFVO incorpora todos los descriptores. NSD, VNFFGD y VLD son incorporados en el catálogo de NS mientras que VNFD es incorporado en el catálogo de VNF como parte de un paquete de VNF. Como resultado del proceso de instanciación, se crean registros para representar las nuevas instancias creadas. Los elementos de información denominados como registro de Servicio de Red (NSR), registro de VNF (VNFR), registro de Enlace Virtual (VLR) y registro de VNFFG (VNFFGR) proporcionan una colección de elementos de datos que pueden ser necesarios para modelar el estado de las instancias de NS, VNF, VNFFG o VL, respectivamente. Estos registros son el resultado de combinar elementos de información NSD, VNFD, VNFFGD y VLD con parámetros de entrada/salida intercambiados en tiempo de ejecución a través de diferentes interfaces producidos y/o consumidos por bloques funcionales NFVMANO.

Durante el ciclo de vida de las instancias respectivas, los registros se actualizan para reflejar los cambios resultantes de la ejecución de las operaciones de gestión del ciclo de vida tanto de servicios de red como 
de VNFs. La Figura 2.14 (b) resume la relación existente entre descriptores y, posteriores instancias y registros del paradigma NFV.

\section{- Modelado de Elementos de Información}

Ciertamente, todas las funcionalidades de gestión y orquestación de servicios de red que proporciona NFV giran en torno a los elementos de información e interfaces propuestas. En el documento de gestión y orquestación propuesto por ETSI [42], se proporcionan detalles de la estructura de estos elementos de información, mostrando en detalle la información que podrían contener cada uno de ellos. Adicionalmente, el documento incluye ejemplos de modelado de elementos de información utilizando diferentes lenguajes o modelos tales como YANG, SID, OVF y TOSCA.

Por otra parte, considerando el nexo existente entre NFV y las tecnologías de computación en la Nube, las plantillas de despliegue para aplicaciones basadas en la Nube también son consideradas como posibles modelos para representar elementos de información de NFV. Chappell [44] resalta que existen diferentes plantillas de despliegue que están tratando de establecerse como estándares en el entorno de la Nube como TOSCA propuesto por OASIS, la plantilla de orquestación Heat (HOT) bajo el desarrollo de la comunidad de OpenStack ${ }^{15}$, las plantillas CloudFormation presentadas por los servicios web de Amazon y Juju Charms de Canonical. También se indica, que existe un creciente interés por TOSCA ya que éste actualmente soporta conceptos más avanzados que Heat y puede ser utilizado por diferentes plataformas de computación en la Nube. Martino et al. [45] proporcionan más detalles sobre las ventajas de TOSCA como la posibilidad de declarar flujos de trabajo gracias al lenguaje imperativo que posee, la definición de un mecanismo de validación y vinculación detallado a través de requisitos y capacidades o la disposición de un conjunto de relaciones predefinidas capaces de vincular componentes de las aplicaciones basadas en la Nube, entre otras.

A continuación, se proporciona una breve descripción de cada uno de estos modelos, haciendo énfasis en TOSCA considerando sus destacadas características.

- YANG. El IETF define a YANG como un lenguaje de modelado de datos utilizado para modelar datos de estado y configuración manipulados por el protocolo de configuración de red (NETCONF) [46]. Con respecto a NETCONF, el IETF indica que es un protocolo que proporciona mecanismos para instalar, manipular y eliminar la configuración de dispositivos de red [9]. YANG se utiliza para modelar dispositivos y servicios de red, es decir, un objeto y sus atributos. Por ejemplo, un dispositivo como un enrutador puede modelarse en YANG y ser configurado a través de NETCONF.

- SID (Shared Information and Data model). Conocido también como Marco de Información (SID) es un componente del proyecto "Frameworx" de TM Forum ${ }^{16}$ el cual tiene como objetivo proporcionar un modelo de información y un vocabulario común para toda la información compartida entre las cosas de interés (entidades) y las relaciones (asociaciones) entre estas entidades.

- OVF (Open Virtualization Format). El $\operatorname{DMTF}^{17}$ (Distributed Management Task Force) ha desarrollado la especificación de OVF [47] con el objetivo de estandarizar un formato abierto, seguro, eficiente y extensible para empaquetar y distribuir software que se ejecutará en sistemas virtuales. OVF se basa en varios estándares del modelo de información común (CIM) definido también por DMTF y permite la creación de sistemas virtuales portátiles y el transporte de sistemas virtuales entre plataformas de virtualización. Considerando la amplia adopción de OVF y CIM en aplicaciones basadas en la Nube, se los puede considerar como punto de partida en el desarrollo de estándares para describir VNFs, en particular, el elemento unidad de despliegue virtual (VDU).

- Juju Charms. Juju ${ }^{18}$ es desarrollado y ofrecido por Canonical, como una aplicación para el fácil despliegue y administración de aplicaciones pre-configuradas y pre-definidas. Juju utiliza una

\footnotetext{
${ }^{15} \mathrm{https}: / /$ www.openstack.org/

${ }^{16} \mathrm{https} / / /$ www.tmforum.org/information-framework-sid/

${ }^{17} \mathrm{https} / / /$ www.dmtf.org/standards/ovf

${ }^{18} \mathrm{https} / / /$ jujucharms.com/
} 
colección de componentes de software denominados "Charms" los cuales contienen todas las instrucciones necesarias para implementar y configurar aplicaciones basadas en la Nube. Cada "Charm" es un paquete estructurado de archivos que describen la configuración (normalmente codificada en YAML ${ }^{19}$ ) y los scripts de acciones (denominados hooks) para gestionar operaciones del ciclo de vida tales como instalación, escalado, actualización, etc. Juju en sí se considera más adecuado como un VNFM, pero un "Charm" podría ser utilizado para definir los descriptores de NFV.

- HOT (Heat Orchestration Template). HOT $^{20}$ representa una de las plantillas soportadas por $\mathrm{Heat}^{21}$. En el programa de orquestación de OpenStack, Heat es el proyecto principal el cual implementa un motor de orquestación para lanzar múltiples aplicaciones compuestas basadas en la Nube utilizando plantillas en forma de archivos de texto que pueden ser tratadas como código. Heat soporta nativamente el formato CFN compatible con CloudFormation, y HOT ha sido diseñado para reemplazarlo. HOT está codificado en YAML y utiliza una forma muy sencilla para representar datos.

- TOSCA (Topology and Orchestration Specification for Cloud Applications). Lipton [48] indica que la portabilidad de servicios que se despliegan en infraestructuras basadas en la Nube es una necesidad en aumento, principalmente para eliminar el efecto de dependencia de un proveedor en particular y disminuir los costos de diseño, despliegue y mantenimiento. Para enfrentar este desafío, se ha propuesto que la portabilidad y la capacidad de gestión de un servicio que se ejecuta en una infraestructura en la Nube sigan un enfoque basado en estándares. Es así, que OASIS ha definido un comité técnico denominado como TOSCA para abordar la especificación del estándar requerido. Garay et al. [43] indican que TOSCA define un lenguaje y un metamodelo para describir servicios, sus componentes, relaciones y procedimientos de gestión. Los principales elementos que definen un servicio se representan en la Figura 2.15 (a) y se listan a continuación:

a. Una plantilla de topología define la estructura de un servicio como un conjunto de plantillas de nodos y plantillas de relación que definen conjuntamente el modelo de topología como un grafo dirigido (no necesariamente conectado).

b. Las plantillas de nodo y relación especifican las propiedades y las operaciones (a través de interfaces) disponibles para manipular el componente. Las relaciones enlazan nodos diferentes y pueden tener diversos significados (por ejemplo, una relación entre un nodo "motor de proceso" y un nodo "servidor de aplicaciones" podría significar "alojado por").

c. Los planes definen los modelos de proceso que se utilizan para crear y terminar un servicio, así como para administrar un servicio durante toda su vida útil.

El perfil NFV de TOSCA [40] especifica un modelo de datos para NFV utilizando el lenguaje TOSCA en consonancia con los principios definidos por ETSI. La Figura 2.15 (b) muestra la relación de correspondencia entre los elementos que componen la plantilla de servicio de TOSCA y los elementos de información de NFV, la misma que puede resumirse de la siguiente manera:

a. NSD se describe utilizando una plantilla de servicio.

b. VNFD, VNFFGD, VLD y PNFD son consideradas como plantillas de nodo con tipos de nodo apropiados.

c. VNFD se puede describir adicionalmente utilizando otra plantilla de servicio con un tipo de nodo sustituible.

La Figura 2.16 muestra un ejemplo de un descriptor de servicio de red (NSD) implementado mediante el perfil NFV de TOSCA. El servicio de red está compuesto por VNF1 y VNF2. Cada VNF está descrita como una plantilla de nodo, cuyo tipo se sustituye por una plantilla de servicio diferente. VNFD1 y VNFD2 son plantillas de servicio que describen los requisitos de comportamiento operacional de cada VNF en detalle e incluidas en el NSD. Como se puede

\footnotetext{
${ }^{19}$ YAML es un formato de serialización de datos legible por humanos. http://yaml.org/

${ }^{20} \mathrm{https}$ //docs.openstack.org/heat/latest/template_guide/hot_spec.html\#hot-spec

${ }^{21} \mathrm{https}: / /$ wiki.openstack.org/wiki/Heat
} 
observar, el NSD permite la composición dinámica de servicios de red, integrando diferentes VNFs y especificando su comportamiento en conjunto.

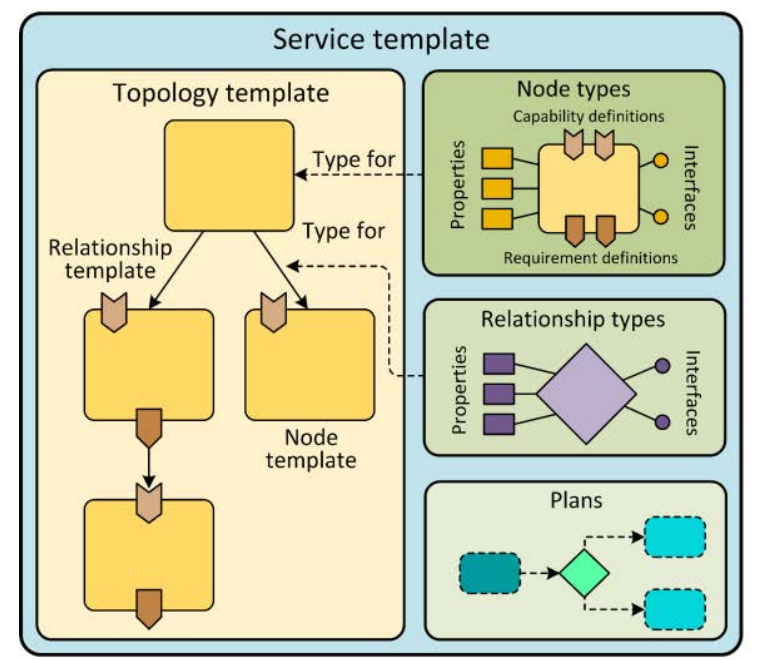

(a)

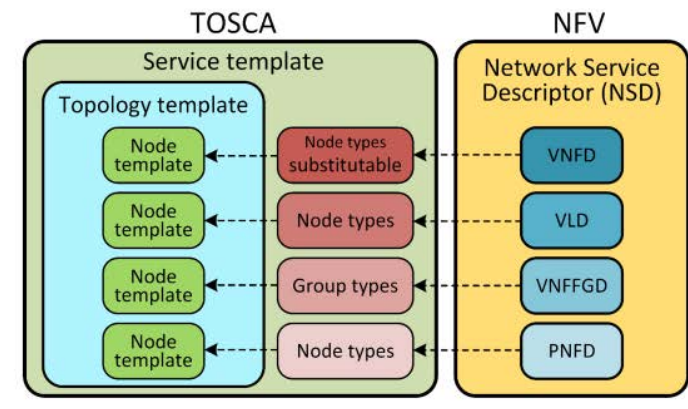

(b)

Figura 2.15 Plantilla de servicio de TOSCA: (a) Elementos y las relaciones entre ellos ${ }^{22}$, (b) Establecimiento de la correspondencia entre la plantilla de servicio definida en TOSCA y el descriptor de servicio de red definido en NFV [40].

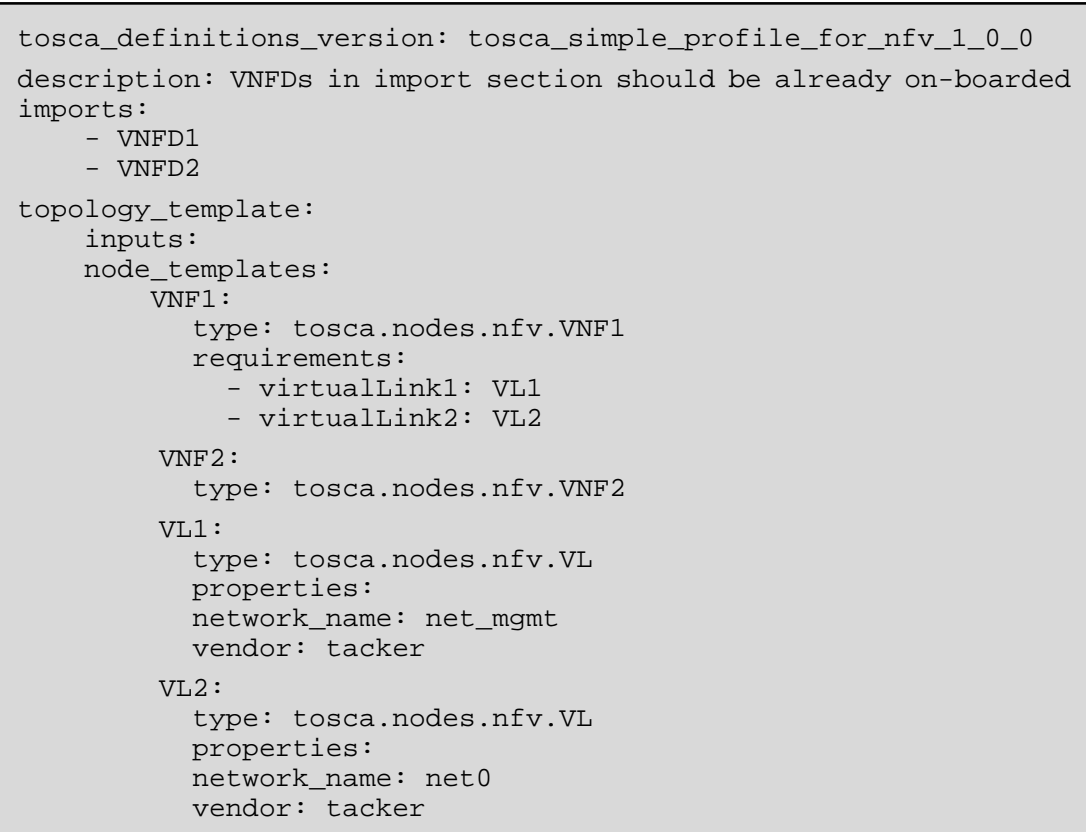

Figura 2.16 Descripción de un servicio de red mediante el perfil NFV de TOSCA. Los enlaces virtuales (VLs) requeridos por VNF1 son proporcionados por NSD mediante un proceso de substitución. Todos los archivos de descripción utilizados tienen la extensión yaml.

Mijumbi et al. [36] proporcionan un cuadro comparativo de los diferentes modelos, sin incluir HOT ni Juju Charms, en el cual se destaca su objetivo, campo de aplicación, desafíos de investigación, entre otros. Los autores también puntualizan que los modelos mencionados no fueron desarrollados considerando los requisitos específicos del paradigma NFV, por tal motivo se los ha adaptado de alguna manera y se espera que evolucionen con el tiempo. Por ejemplo, YANG proporciona una forma de describir "qué es un servicio", sin describir "cómo se debe implementar un servicio", la topología de los

\footnotetext{
${ }^{22}$ http://docs.oasis-open.org/tosca/TOSCA/v1.0/os/TOSCA-v1.0-os.html
} 
recursos subyacentes o cómo los recursos pueden estar relacionados o dependientes unos de otros. Por otra parte, TOSCA es un lenguaje estándar creado específicamente para la orquestación. Sin embargo, TOSCA no proporciona una interfaz de configuración que permita controlar las operaciones del ciclo de vida de las instancias desplegadas en tiempo de ejecución. Para solventar este inconveniente, se pueden combinar soluciones para compensar funcionalidades faltantes como lo propone Chappell [44]. Así, TOSCA y su modelo de despliegue centrado en las aplicaciones permitiría desplegar instancias de VNF con un conjunto inicial de configuraciones en la NFVI mientras que un API de configuración basada en modelos como NETCONF/YANG permitiría realizar reconfiguraciones específicas del servicio a la instancia de VNF en tiempo de ejecución.

\subsubsection{Interfaces NFV-MANO para la Gestión y Despliegue de Servicios de Red}

El principal objetivo que plantea el paradigma NFV es proporcionar una gestión ágil y automatizada de un determinado servicio de red y las diferentes VNFs que lo componen a través de las operaciones de sus ciclos de vida. De acuerdo con el documento de Gestión y Orquestación de NFV propuesto por ETSI [42], las operaciones del ciclo de vida se realizan en los elementos de información, es decir, sobre los descriptores y posteriores registros o instancias. Para este fin, NFV-MANO proporciona diferentes interfaces relativas a los servicios de red, VNFs, recursos virtualizados, administración de políticas, gestión del hipervisor de NFVI, entre otros. A continuación, de proporciona una breve descripción de las interfaces para servicios de red y VNFs.

\section{- Interfaces para Servicios de Red}

Dentro de este tipo, se diferencian las interfaces orientadas a descriptores e instancias. Con respecto a la gestión del NSD, la interfaz permite realizar diferentes operaciones como incorporar/eliminar, habilitar/deshabilitar descriptores del catálogo de NS. También se permite actualizar el contenido de los descriptores para reflejar algún cambio en su estructura, así como consultar información relativa a los mismos como la política de escalamiento, el sabor de despliegue del servicio, listar VNFs participantes, etc. Es importante mencionar que cualquier operación realizada con el NSD puede requerir realizar una operación similar en cualquier VNFFGD y VLD relacionado con el objetivo de mantener la coherencia en el servicio de red.

Con respecto a las instancias de NS, se proporcionan interfaces para la gestión del ciclo de vida, notificación de cambios en el ciclo de vida, gestión del rendimiento y gestión de fallos. Las interfaces en conjunto proporcionan opciones variadas para gestionar las instancias de NS, incluyendo la gestión de las asociaciones entre diferentes VNFs, y de VNFs cuando están conectados a PNFs, la topología del servicio de red y los grafos de envío de VNF asociados con el servicio. Por ejemplo, la operación "actualizar" el servicio de red puede desencadenar la instanciación de una VNF adicional y la correspondiente "actualización" de la instancia existente de VNFFG asociada al servicio. Es importante mencionar que las operaciones relativas a la gestión del ciclo de vida de NS se las realiza en tiempo de ejecución sin la necesidad de detener la instancia de NS y posteriormente re-iniciarla una vez que los descriptores involucrados hayan sido modificados para reflejar los cambios requeridos.

\section{- Interfaces para VNFs}

Como se ha mencionado anteriormente, una VNF está asociada a un paquete y, por lo tanto, se proporciona una interfaz para gestionarlo, incluyendo el VNFD y la imagen de software requerida. Esta interfaz permite incorporar/eliminar, habilitar/deshabilitar, actualizar o consultar información de un paquete.

Con respecto a la instancia de VNF, se proporcionan diferentes interfaces destinadas a gestionar el ciclo de vida, notificar cambios en el ciclo de vida, configurar la VNF, gestionar el rendimiento de la VNF y gestionar eventuales fallos. Las capacidades de gestión de cada interfaz están definidas por el conjunto de operaciones asociadas a la misma. Por ejemplo, la interfaz de gestión del ciclo de vida de la instancia de VNF incluye operaciones para ejecutar una actualización de software limitada o desplegar una nueva versión de software con el objetivo de mejorar las funcionalidades proporcionadas. Adicionalmente, esta interfaz incluye una operación para modificar la estructura de la instancia, como la topología, 
comportamiento o modelo de redundancia, con el objetivo de cumplir con los requerimientos de las nuevas funcionalidades incorporadas o mejorar el rendimiento.

Es importante mencionar que cada instancia de VNF expone una interfaz de configuración. Esta interfaz proporciona operaciones destinadas a manipular los parámetros de configuración de una VNF los cuales son representados mediante "objetos de configuración". En este sentido, si el VNFM consume esta interfaz, se pueden pasar parámetros de configuración a la VNF sin necesidad de tener en cuenta su semántica.

\subsubsection{Plataformas de NFV}

La adopción y evolución del paradigma NFV depende de dos factores fundamentales. Por una parte, el desarrollo de estándares que garanticen la interoperabilidad de las soluciones basadas en NFV y, por otra parte, plataformas NFV que satisfactoriamente implementen el marco arquitectónico de NFV considerando los requisitos específicos de un entorno de operador de red. Con respecto a los estándares, ETSI conjuntamente con otros Organismos de Desarrollo de Estándares como el IETF están a cargo de la estandarización de NFV, logrando hasta el momento avances significativos en el tema. Con respecto al desarrollo de plataformas NFV, varias iniciativas propietarias y de código abierto han sido presentadas. Cada una de estas propuestas trata de implementar el marco de gestión y orquestación (MANO) propuesto por ETSI desde su perspectiva, dando como resultado un alto grado de heterogeneidad en las plataformas NFV. Diferentes autores realizan un estudio detallado de las plataformas NFV existentes con el objetivo de analizar sus características y funcionalidades proporcionadas.

Por ejemplo, Mijumbi et al. [36] presentan algunos proyectos de NFV considerando el objetivo del proyecto, el área de interés, entidades que lo lideran o financian y el tipo de proyecto. En el mismo artículo también se presentan implementaciones de NFV realizadas por la industria considerando la funcionalidad, las entidades de estandarización en las cuales se basan y las plataformas en las cuales se ejecutan (OpenStack, OpenDayLight, etc.). Mijumbi et al. [49] en un artículo distinto, presentan como diferentes proyectos y productos NFV se basan en infraestructuras existentes como OpenStack, algunos de ellos utilizan TOSCA para modelar los elementos de información de NFV y la mayoría propone una solución para los bloques funcionales de MANO. En el mismo artículo también se presenta las capacidades de gestión y orquestación de cada proyecto/producto considerando cuatro categorías principales: enfoque de gestión, función de gestión (FCAPS), la orientación de la gestión (funciones, servicios o red) y las áreas relacionadas de gestión (SDN o Cloud).

Chayapathi et al. [31] realizan una distinción entre soluciones de software, propietarias y de código abierto, que desempeñan solamente funciones de VNFM, otras enfocadas en el despliegue y orquestación de Servicios de Red y otras enfocadas en proporcionar la funcionalidad de los bloques de MANO completa. Similarmente, Gil y Botero [50] realizan una descripción de algunos proyectos NFV realizados por la industria y la comunidad académica. Posteriormente, realizan una clasificación considerando si el proyecto es o no de código abierto, los componentes del marco arquitectónico de NFV implementados, la entidad que lidera el proyecto y la principal contribución.

De la revisión del estado del arte se puede decir que, si bien la mayoría de plataformas NFV presentadas comparten el objetivo común de implementar el marco arquitectónico de NFV propuesto por ETSI, las funcionalidades o capacidades reales de gestión y orquestación son proporcionadas con un cierto grado de limitación. Por ejemplo, algunas implementaciones de NFV se limitan a controlar las operaciones del ciclo de vida de VNFs sin considerar los NSs. Por otra parte, existen implementaciones que permiten controlar las operaciones del ciclo de vida de los descriptores, pero no proporcionan un conjunto completo de operaciones del ciclo de vida para las instancias. Ciertamente, este es el resultado esperado considerando que NFV es una tecnología relativamente nueva que debe evolucionar para proporcionar todas las funcionalidades de gestión y orquestación propuestas por ETSI y requeridas por los operadores de red.

Adicionalmente, se observa una tendencia en la creación de consorcios formados por operadores de red, fabricantes de equipos de red y academia para el desarrollo de plataformas de código abierto enfocadas 
en consolidar el paradigma NFV. SDX central ${ }^{23}$ en su reporte sobre NFV de 2017 [51] indica que a medida que los proyectos de código abierto evolucionan, los proveedores de tecnología también se están posicionando, tomando prestadas algunas tecnologías de código abierto y presentando su propia mejora a través de la integración. El resultado es una explosión de diferentes enfoques y tecnologías competitivas. En este reporte se destacan las plataformas de NFV: Open Source MANO, OpenBaton, Tacker, OPNFV y ONAP.

A continuación, se proporciona una breve descripción de las plataformas NFV, propietarias y de código abierto, más relevantes en la actualidad. En la sección siguiente, se incluye una descripción detallada de Tacker considerando que es un proyecto propio de OpenStack y con el objetivo de ilustrar cómo una plataforma en particular implementa el marco arquitectónico NFV de ETSI.

- Orquestador de Servicios de Red (NSO) de Cisco $^{24}$. Se proporciona un paquete de funciones principales NFVO, el cual combina el orquestador de servicios de red con la lógica de orquestación NFV, los modelos de datos ETSI NFV y el controlador de servicios elástico (ESC) encargado de la gestión del ciclo de vida de las VNFs. Se utiliza un esquema de descriptor basado en YANG que permite crear descriptores de servicio de red e incorporar VNFs de distintos proveedores utilizando JSON, XML o TOSCA/YAML. De esta manera se proporciona una plataforma versátil e independiente de proveedores utilizable con redes físicas, virtuales o híbridas que permite controlar por completo las operaciones del ciclo de vida de las instancias de VNF y NS en ejecución.

- Open Platform NFV (OPNFV) ${ }^{25}$. Es un proyecto de código abierto fundado y alojado por la fundación Linux y, compuesto por operadores de telecomunicaciones y proveedores. Su objetivo es establecer una plataforma de referencia de código abierto, adaptada al entorno del operador, para avanzar en la evolución de NFV. OPNFV proporciona NFVI y VIM mediante la integración de componentes de proyectos preliminares como OpenDaylight, ONOS, OpenStack, Ceph, KVM, Open vSwitch y Linux. Si bien no es específicamente una solución MANO, representa una solución integrada de NFV centrada en la verificación y la garantía. El lanzamiento reciente de OPNFV Danube ofrece metodologías DevOps a NFV a través del desarrollo preliminar colaborativo, la integración, la implementación y la importante automatización de pruebas de integración continua/desarrollo continuo $(\mathrm{CI} / \mathrm{CD})$.

- Open Source MANO (OSM) ${ }^{26}$. Fue presentado en febrero de 2016 con el objetivo de ofrecer una funcionalidad de código abierto MANO que cumpla plenamente con el marco de ETSI. OSM ha recibido contribuciones de Telefónica y su proyecto OpenMANO, Canonical y su VNFM genérico Juju y RIFT.io con su orquestador de NFV de código abierto. La comunidad de OSM ha definido un alcance para el proyecto que abarca tanto los aspectos de tiempo de diseño como de ejecución relacionados con la prestación de servicios para entornos de proveedores de servicios de telecomunicaciones. El lanzamiento de la versión tres de OSM se enfoca en aspectos como la usabilidad de la plataforma, mejoras en el modelamiento de VNFs y NSs, configuración de VNF extremadamente flexible y una gestión avanzada de redes, así como capacidades de seguridad mejoradas con controles de acceso avanzados, entre otros.

- T-NOVA ${ }^{27}$. Con el objetivo de promover el concepto de NFV, T-NOVA presenta un novedoso marco facilitador, que permite a los operadores no solo desplegar funciones de red virtualizadas para sus propias necesidades, sino también ofrecerlas a sus clientes, como servicios de valor agregado. Kourtis et al. [52] indican que las funcionalidades MANO de T-NOVA se basan en la plataforma de orquestación de NFV "TeNOR" y sus módulos actualmente implementados que soportan la gestión de cuatro fases críticas del ciclo de vida del servicio denominadas como descubrimiento de recursos, mapeo de servicios, despliegue de servicios y supervisión. A su vez, la funcionalidad de NFVO

\footnotetext{
${ }^{23} \mathrm{https}: / / \mathrm{www} . \mathrm{sdxcentral.com/}$

${ }^{24} \mathrm{https} / /$ www.cisco.com/c/en/us/solutions/service-provider/solutions-cloud-providers/network-services-orchestrator-

solutions.html

${ }^{25} \mathrm{https}$ ://www.opnfv.org/

${ }^{26} \mathrm{https}$ ///osm.etsi.org/

${ }^{27}$ http://www.t-nova.eu/
} 
proporcionada por TeNOR es dividida en dos submódulos principales: Orquestador de Servicio de Red (NSO) y Orquestador de Recursos Virtualizados (VRO).

- OpenBaton ${ }^{28}$. Esta plataforma permite la implementación de NSs sobre infraestructuras heterogéneas de NFV, se integra con OpenStack y proporciona un mecanismo basado en plugins para integrar otros tipos de VIM. Soporta la gestión de NS ya sea utilizando un VNFM genérico o interactuando con un VNFM específico. Utiliza diferentes mecanismos (RESTful o APIs Pub/Sub) para interactuar con los VNFMs y también se integra con componentes adicionales para la gestión en tiempo de ejecución de un NS. Por ejemplo, se proporciona escalamiento automático y gestión de fallas en función de la información de supervisión proveniente del NFVI.

- Open Network Automation Platform (ONAP) ${ }^{29}$. ONAP es un proyecto de la fundación Linux que reúne los proyectos Open ECOMP y Open-O como una plataforma integral para la orquestación y automatización en tiempo real, basada en políticas, de funciones de red físicas y virtuales que permitirán a los proveedores y desarrolladores de software, redes, TI y Cloud crear rápidamente nuevos servicios. Por una parte, Open ECOMP principalmente proporciona un marco orientado al tiempo de diseño utilizado para el diseño y la composición de servicios de red, políticas y VNFs. Por otra parte, Open-O proporciona un marco orientado al tiempo de ejecución que implementa todas las funcionalidades de orquestación.

\subsubsection{Tacker}

Tacker $^{30}$ es un proyecto oficial de OpenStack que implementa un VNFM genérico y un NFVO para desplegar y operar NSs y VNFs en una infraestructura de NFV como OpenStack. Se basa en NFVMANO de ETSI y proporciona un conjunto funcional de componentes para orquestar servicios de red de extremo a extremo utilizando VNFs.

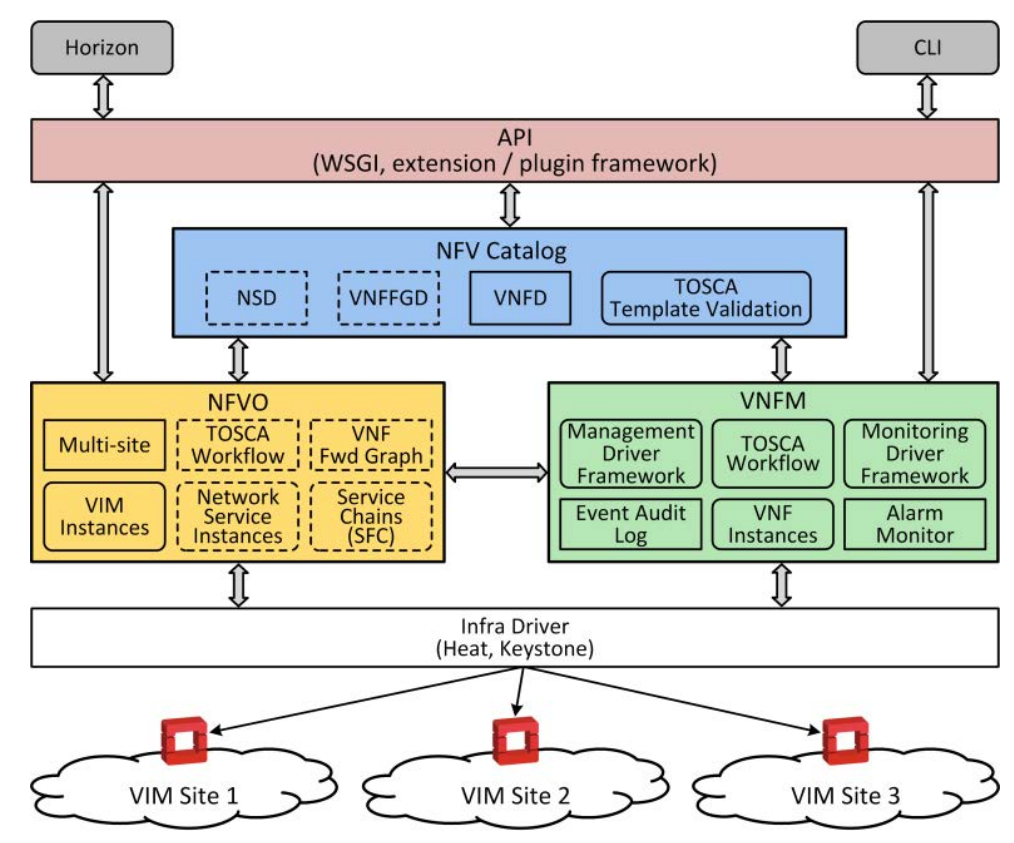

Figura 2.17 Arquitectura de alto nivel de Tacker.

\section{- Arquitectura}

La Figura 2.17 muestra la arquitectura de alto nivel de Tacker compuesta por los siguientes componentes:

\footnotetext{
${ }^{28} \mathrm{https}: / /$ openbaton.github.io/

${ }^{29} \mathrm{https}$ ://www.onap.org/

${ }^{30} \mathrm{https}: / /$ wiki.openstack.org/wiki/Tacker
} 
a. Catálogo de NFV. Representa un repositorio de descriptores para servicios de red (NSD), VNFs (VNFD) y grafos de envío de VNF (VNFFGD), los cuales son especificados mediante plantillas de servicio TOSCA y almacenados en una DB.

b. Gestor de VNF (VNFM). Encargado de gestionar los procesos relacionados al ciclo de vida de las VNFs (Create, Read, Update, Delete, CRUD) y facilitar su configuración inicial. Similarmente, se encarga del monitoreo, auto recuperación y escalado automático de VNFs desplegadas en base a políticas.

c. Orquestador de NFV (NFVO). Permite generalizar, mediante plantillas, el despliegue de NSs de extremo a extremo. Se encarga de verificar y asignar recursos sobre el VIM, asegurando una eficiente colocación de las VNFs mediante políticas.

d. API. Tacker exporta sus capacidades actuales mediante un API, el cual es accesible mediante la línea de comandos (CLI), el Dashboard (Horizon) o remotamente a través de REST. A través del API, se controla el ciclo de vida de descriptores y posteriores instancias de VNFs, NSs y VNFFGs. Los APIs proporcionados se corresponde con las interfaces NFV-MANO definidas por ETSI.

\section{- Interfaces de programación de aplicaciones (APIs)}

De acuerdo con la documentación de Tacker $^{31}$ se proporcionan APIs encargadas de controlar las operaciones CRUD de descriptores y posteriores instancias. Se distinguen tres tipos de APIs: el NFVO NS API orientado a los servicios de red (NSs), el VNFM API orientado a las funciones de red virtualizadas (VNFs) y el VNFFG API orientado a las cadenas de función de servicio (SFCs) o grafos de envío de VNF (VNFFG). La Tabla 2.1, Tabla 2.2 y Tabla 2.3 muestran las opciones que actualmente incorporan las diferentes APIs. En la documentación de Tacker se proporciona información adicional como los formatos de solicitud y respuesta de las operaciones realizadas.

\section{- Flujo de trabajo}

Las operaciones de gestión y orquestación de NFV efectuadas por Tacker, se controlan a través del API que se proporciona. El API es utilizado por diferentes entidades como los OSS/BSS o un orquestador de NFV (NFVO) distinto con el objetivo de desplegar servicios de red sobre una plataforma NFV. La Figura 2.18 muestra un ejemplo del flujo de trabajo para gestionar VNFs y VNFFGs, el cual incluye siete etapas fundamentales.

- En la primera etapa, a través del VNFM API, diferentes clientes pueden realizar operaciones CRUD sobre descriptores (VNFDs), las cuales posteriormente son reflejadas en el catálogo de NFV.

- En la segunda etapa, se procede a instanciar una VNF específica en la NFVI. Para este fin, se utiliza la opción "Create VNF" del VNFM API, especificando el Id del VNFD recuperado previamente desde el catálogo. El despliegue de la VNF está controlado por diferentes procesos que ejecuta el VNFM y el NFVO. Como los descriptores están especificados en TOSCA, se realiza un proceso de conversión al formato de plantilla de despliegue utilizado por Heat ya que éste está encargado de gestionar el ciclo de vida de las máquinas virtuales (VMs) en OpenStack.

- En la tercera etapa, se procede a configurar la VNF previamente instanciada utilizando para ello el marco de controladores de gestión (Mgmt Driver). El controlador de gestión utiliza un enfoque de selección de servicios, permitiendo especificar una configuración inicial la cual puede ser actualizada cuando la VNF se encuentra activa.

- En la cuarta etapa, Tacker inicia el proceso de monitorización de las condiciones de estado de todas las VNFs que se encuentran operativas con el objetivo de detectar fallos y ejecutar procesos de recuperación.

\footnotetext{
${ }^{31} \mathrm{https} / / /$ developer.openstack.org/api-ref/nfv-orchestration/v1/
} 
Tabla 2.1 NFVO NS API.

\begin{tabular}{|l|l|l|}
\hline \multicolumn{1}{|c|}{ URI } & Method & \multicolumn{1}{c|}{ API } \\
\hline$/ \mathrm{v} 1.0 / \mathrm{nsd}$ & GET & List $n s d s:$ lists NSD names/IDs \\
\hline$/ \mathrm{v} 1.0 / \mathrm{nsd} /<\mathrm{nsd}$ id $>$ & GET & Show $n s d:$ shows information of a specific NS \\
\hline$/ \mathrm{v} 1.0 / \mathrm{nsd}$ & POST & Create $n s d:$ creates a NSD \\
\hline$/ \mathrm{v} 1.0 / \mathrm{nsd} /<\mathrm{nsd}$ id $>$ & DELETE & Delete $n s d:$ deletes a specific NSD \\
\hline$/ \mathrm{v} 1.0 / \mathrm{ns}$ & GET & List $n s^{\prime}:$ lists instantiated NS' in NFV Orchestrator \\
\hline$/ \mathrm{v} 1.0 / \mathrm{ns} /<\mathrm{ns}$ id $>$ & GET & Show $n s:$ shows information of a specific NSD \\
\hline$/ \mathrm{v} 1.0 / \mathrm{ns}$ & POST & Create $n s:$ creates a NS based on the ID \\
\hline
\end{tabular}

Tabla 2.2 VNFM API.

\begin{tabular}{|c|c|c|}
\hline URI & Method & API \\
\hline /v1.0/vnfds & GET & List vnfds: lists VNFDs stored in the catalog \\
\hline /v1.0/vnfds/<vnfd_id $>$ & GET & Show $v n f d$ : shows information for a specified VNFD \\
\hline /v1.0/vnfds & POST & Create vnfd: creates a VNFD entry based on the vnfd template \\
\hline /v1.0/vnfds/<vnfd_id $>$ & PUT & Update vnfd: updates a given VNFD \\
\hline /v1.0/vnfds/<vnfd_id $>$ & DELETE & $\begin{array}{l}\text { Delete } v n f d \text { : Deletes a given VNFD. Only a VNFD without } \\
\text { any associated VNFs can be deleted. }\end{array}$ \\
\hline /v1.0/vnfs & GET & List $v n f s:$ lists instantiated VNFs \\
\hline /v1.0/vnfs/<vnf_id $>$ & GET & Show vnf: shows information of a given VNF \\
\hline /v1.0/vnfs & POST & Create vnf: creates a VNF based on the VNFD \\
\hline /v1.0/vnfs/<vnf_id $>$ & PUT & Update vnf: updates a VNF based on user config file or data \\
\hline /v1.0/vnfs/<vnf_id $>$ & DELETE & Delete vnf: deletes a given VNF \\
\hline$/ \mathrm{v} 1.0 /$ vnfs/ $/$ vnf_id $>/$ resources & GET & $\begin{array}{l}\text { List VNF resources: Lists resources, such as VDU/CP, of a } \\
\text { given VNF. }\end{array}$ \\
\hline /v1.0/vnfs/<vnf_id $>$ /actions & POST & Trigger VNF scaling: Triggers VNF scaling action. \\
\hline
\end{tabular}

Tabla 2.3 VNFFG API.

\begin{tabular}{|c|c|c|}
\hline URI & Method & API \\
\hline /v1.0/vnffgd & GET & List vnffgds: lists VNFFGDs stored in the catalog \\
\hline$/$ v1.0/vnffgd/<vnffgd_id $>$ & GET & Show vnffgd: shows information of a given VNFFGD \\
\hline /v1.0/vnffgd & POST & Create vnffgd: creates a VNFFGD \\
\hline /v1.0/vnffgd/<vnffgd_id $>$ & DELETE & $\begin{array}{l}\text { Delete vnffgd: deletes a given VNFFGD. Only a VNFFGD } \\
\text { without any associated VNFFGs can be deleted. }\end{array}$ \\
\hline /v1.0/vnffg & GET & List vnffgs: lists instantiated VNFFGs \\
\hline$/$ v1.0/vnffg/<vnffg_id $>$ & GET & Show vnffg: shows information of a given VNFFG \\
\hline /v1.0/vnffg & POST & Create vnffg: creates a VNFFG \\
\hline /v1.0/vnffg/<vnffg_id $>$ & DELETE & Delete vnffg: deletes a given VNFFG \\
\hline /v1.0/nfps & GET & List $n f p s$ : lists NFPs \\
\hline$/ \mathrm{v} 1.0 / \mathrm{nfps} /<\mathrm{nfp} \_\mathrm{id}>$ & GET & Show $n f p$ : shows information of a given NFP \\
\hline$/ \mathrm{v} 1.0 / \mathrm{sfcs}$ & GET & List SFCs: lists active SFCs \\
\hline$/ \mathrm{v} 1.0 / \mathrm{sfcs} /<\mathrm{sfc}$ id $>$ & GET & Show SFC: shows information of a given SFC \\
\hline /v1.0/classifiers & GET & List classifiers: lists classifiers \\
\hline /v1.0/classifiers/<sfc_id $>$ & GET & Show classifier: shows information of a given classifier \\
\hline
\end{tabular}




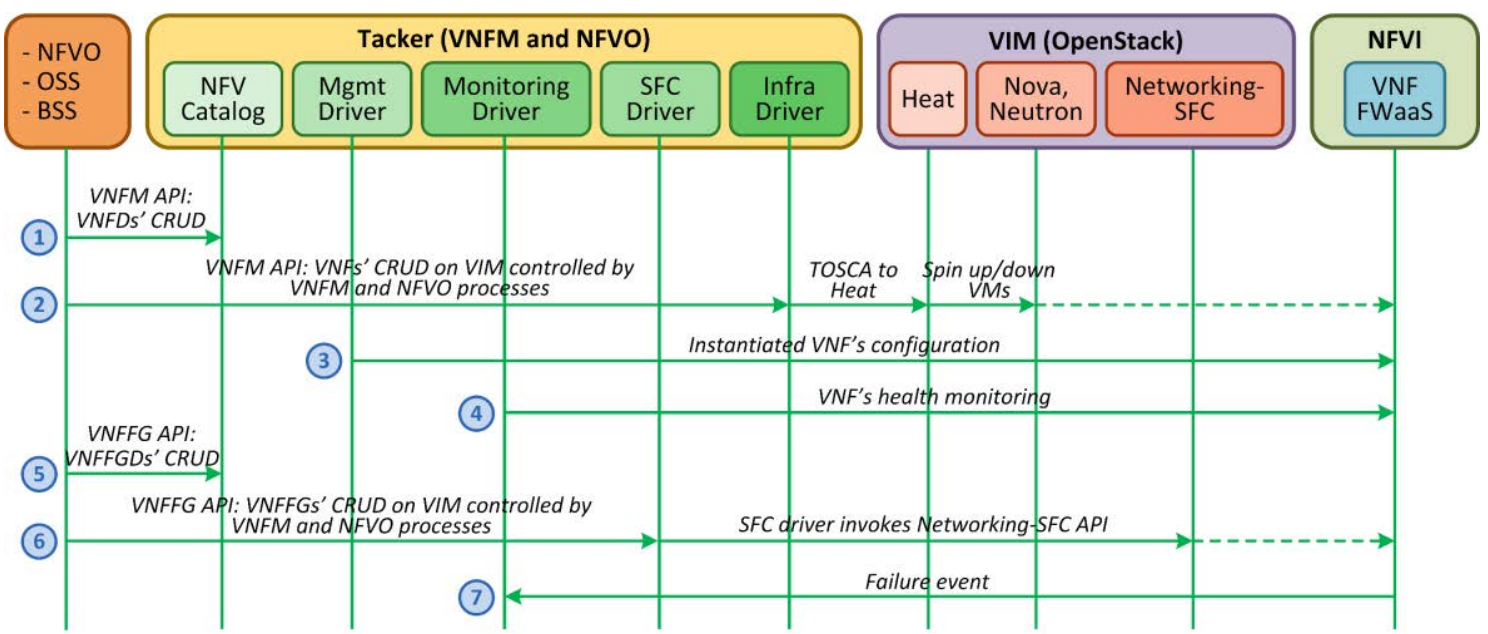

Figura 2.18 Flujo de trabajo de Tacker. Las operaciones CRUD de descriptores e instancias desplegadas son controladas mediante los APIs NFVO NS, VNFM y VNFFG.

- En la quinta etapa se procede a crear VNFFGs con diferentes VNFs que se encuentren en estado operacional. El proceso a seguir es similar al utilizado en el despliegue de VNFs. Para ello, primero se debe crear un descriptor de VNFFG (VNFFGD) para incorporarlo al catálogo de NFV. Esto se consigue mediante el VNFFG API que permite especificar las operaciones CRUD relacionadas.

- En la sexta etapa se procede a crear una instancia de VNFFG mediante la creación de la ruta de envío de red correspondiente y el clasificador requerido sobre la NFVI subyacente. Para realizar esta y otras operaciones CRUD también se utiliza el VNFFG API. Como se puede ver en la Figura 2.18, Tacker incorpora un controlador de SFC destinado a encadenar las VNFs especificadas en el descriptor utilizando para ello el Networking-SFC API que proporciona Neutron en OpenStack de forma nativa.

- En la etapa final, se considera un eventual fallo de una VNF. En la política de monitorización asociada a la VNF se especifica la acción a ser ejecutada cuando se detecta un fallo. Por ejemplo, se podría solicitar que se re-genere (re-spawning) la VM cuando no existe respuesta desde la VNF o ejecutar un proceso de escalamiento automático (auto scaling) para agregar o eliminar (scale out/in) instancias de VNF cuando la CPU de la VM alcanza un determinado umbral.

\subsection{SDN, NFV y Virtualización Aplicadas a las Redes Residenciales}

Hoy en día, la Virtualización, Virtualización de Funciones de Red (NFV) y Redes Definidas por Software (SDN) son tendencias notables en el escenario de los operadores de telecomunicaciones. En lo que respecta a las redes residenciales, se han presentado diferentes propuestas basadas en estas tecnologías que pretenden principalmente: simplificar la gestión de este entorno en respuesta a la creciente complejidad y limitada usabilidad existente, asi como mejorar la conectividad que proporciona la red residencial para que se puedan soportar servicios o aplicaciones avanzadas. La mayoría de estas propuestas tienen el objetivo común de evitar el aumento de costos (CaPex/OpEx) asociados a las modificaciones radicales del diseño de la red del ISP. Si la solución es atractiva para los ISPs en estos términos y, proporciona evidencia para respaldar su viabilidad, será más probable que tenga éxito.

En esta sección, con el objetivo de proponer una solución efectiva y viable que aborde la gestión y provisión de conectividad de red adaptada a las demandas de determinados servicios avanzados en consonancia con las tecnologías antes mencionadas, se realiza un análisis de las propuestas existentes considerando diferentes características. En primer lugar, se hace una clasificación con base en el tipo de tecnología que utilizan, ya sea virtualización, NFV o SDN. En segundo lugar, la participación de los 
usuarios residenciales en las tareas de gestión establece si la propuesta está centrada en el usuario o no. Finalmente, se determina si la propuesta analizada proporciona una solución integral que aborde todos las necesidades de los usuarios residenciales identificados en la sección 2.1.4 (Facilidad de Gestión, Visibilidad, QoS/QoE y Seguridad), o solo algunos de ellos.

\subsubsection{Virtualización y Virtualización de Funciones de Red (NFV)}

La virtualización del RGW es actualmente la principal tendencia en este campo debido a dos razones. En primer lugar, las tecnologías de virtualización han alcanzado un alto nivel de madurez, utilizándose ampliamente para aumentar la eficiencia y la flexibilidad del Centro de Datos. En segundo lugar, la virtualización permite continuar utilizando el paradigma de redes tradicional basado en capas (L1 - L7). En función de estas razones, se consideran dos enfoques para implementar la virtualización del RGW.

El primer enfoque, propone que las funciones de red específicas como el encaminamiento o NAT se ejecuten sobre diferentes instancias virtualizadas dentro del propio RGW. Así, Ibañez et al. [53] proponen la virtualización de la plataforma OSGi que se ejecuta en el RGW con el objetivo de crear múltiples instancias, cada una de las cuales proporciona un conjunto de funciones o servicios definidos y controlados por un ISP específico. Similarmente, Whiteaker et al. [54] definen una Pasarela de Alojamiento de Servicios (Service-hosting Gateway, SHG) que actúa como una plataforma de ejecución mediante la virtualización del sistema operativo donde ésta se ejecuta. Los usuarios deciden los servicios que se ejecutarán en un SHG individual siguiendo un modelo de tienda de aplicaciones y, a través de la virtualización, cada servicio se ejecutará en una máquina virtual separada.

Ciertamente, las propuestas de Ibañez et al. y Whiteaker et al. proporcionan flexibilidad para integrar nuevos servicios o funciones al RGW a través de técnicas de virtualización y permiten que el usuario residencial escoja cuales servicios agregar. Sin embargo, las propuestas aíslan al usuario de las tareas de gestión ya que los servicios son utilizados principalmente por el ISP y además no se especifica si tales servicios tienen como objetivo abordar los requerimientos del usuario residencial. Adicionalmente, debido a los recursos computacionales disponibles limitados del RGW, ambos enfoques presentan una desventaja importante que puede restringir el desempeño de determinados servicios avanzados con altas demandas de recursos computacionales. Además, si los servicios en ejecución exigen más recursos, es obligatoria una actualización de hardware del RGW. Esta opción es poco adecuada, ya que representa un incremento sustancial de CaPex y, en consecuencia, los procedimientos correspondientes con respecto a la implementación, el mantenimiento y la gestión pueden ser problemáticos.

El segundo enfoque, propone externalizar las funciones de red del RGW. Cruz et al. [55], presentan una arquitectura para virtualizar el RGW. La mayoría de las funciones del RGW se mueven a la infraestructura del operador utilizando virtualización y nubes privadas mientras se mantiene un dispositivo drásticamente simplificado en las instalaciones del usuario. Este enfoque está estrechamente relacionado con el paradigma de virtualización de funciones de red (NFV) analizado en la sección 2.3 $\mathrm{y}$, en particular, con el caso de uso de aplicabilidad propuesto orientado a la virtualización del entorno residencial [56]. En este escenario, un dispositivo L2 (capa 2) reemplaza al RGW estándar y las funciones restantes de nivel superior, como el encaminamiento o NAT, se proporcionan como Funciones de Red Virtualizadas (VNFs) en la infraestructura de NFV (NFVI) del ISP.

Broadband Forum con base en el enfoque de ETSI para virtualizar el RGW propone la arquitectura NERG (Network Enhanced Residential Gateway) [57]. En esta arquitectura se define el gateway virtual (vG) como un componente lógico encargado de contener las funciones de red que son externalizadas del RGW estándar. Se plantea que un MS-BNG (Multi-Service Broadband Network Gateway) o una NFVI asociada se encargue de alojar a los diferentes vGs y sus correspondientes funciones, ya sean VNFs o PNFs. NERG también plantea el caso en el que un determinado tráfico en el vG pueda necesitar utilizar un diferente grupo de servicios o cadena de servicio para lo cual se debería implementar un direccionamiento selectivo y clasificación de tráfico. Broadband Forum desarrolla un poco más esta idea en TR-345 [58] donde se propone implementar una NFVI para aumentar o reemplazar los servicios proporcionados por el BNG con VNFs. En este escenario, una función de clasificación localizada en un MS-BNG físico es responsable de direccionar el tráfico del suscriptor a un grafo de servicio o SFC 
desplegada en el NFVI. A su vez, la asignación se puede aplicar a todo el tráfico de suscriptor o flujos específicos.

En un informe elaborado conjuntamente entre OPNFV y OpenDayLight [59] se indica que la virtualización del CPE (Customer Premises Equipment) se ha convertido en el caso de uso más popular de aplicación de NFV y SDN, destacando que su implementación satisfactoria requiere el uso de SFCs que permitan acceder a otras funciones de red. El informe argumenta que, aunque el CPE virtual (vCPE) es un caso de uso que se puede aplicar tanto en entornos empresariales como residenciales, los requisitos y enfoques para los dos casos son bastante diferentes. Por ejemplo, en el caso de un vCPE empresarial, se podría requerir que una funcionalidad específica se ejecute localmente en la sucursal mientras que, en otro caso con el objetivo de reducir el costo y la complejidad de la sucursal, algunas de las funciones de su CPE podrían implementarse en el centro de datos de la empresa.

En el informe de 2017 sobre Virtual Edge y SD-WAN [60] realizado por SDX central se indica que la tecnología vCPE empresarial puede ejecutar las instancias virtualizadas en las instalaciones del cliente (a veces en equipos de menor costo), en oficinas centrales (CO) o puntos de presencia (PoP) o en centros de datos. Varias arquitecturas vCPE empresariales proporcionan enfoques híbridos que permiten ejecutar diferentes funciones en diferentes ubicaciones para optimizar la latencia, la gestionabilidad y el costo.

La iniciativa CORD ${ }^{32}$ (Central Office Re-architected as a Data Center) [61] aprovecha las tecnologías SDN, NFV y Cloud para proponer un nuevo diseño que transforme la central de telecomunicaciones (CO) en un centro de datos. El objetivo es reemplazar el hardware propietario y cerrado con software que se ejecuta en dispositivos de acceso, conmutadores y servidores genéricos o estándar para mejorar la provisión de servicios de telecomunicaciones. Se ofrecen soluciones orientadas a entornos residenciales (R-CORD), empresariales (E-CORD) y móviles (M-CORD). La propuesta residencial de CORD incluye una Red de Acceso Óptica Definida por Software (SDOAN) compuesta por un OLT (Optical Line Terminal) habilitado para OpenFlow y su complemento de software designado como OLT virtual (vOLT). Al igual que ETSI y Broadband Forum, R-CORD también virtualiza el RGW o CPE, colocando un dispositivo "bare-metal switch" en casa del usuario y desplegando un Gateway de Subscriptor virtual (vSG) ${ }^{33}$ en la Nube de la oficina central que contiene un conjunto de funciones seleccionadas por el usuario. Todos los componentes residenciales de CORD están orquestados por una plataforma de administración centralizada.

De la revisión realizada se observa un claro consenso en cuanto a la propuesta de virtualización del RGW (vRGW) basada en el paradigma NFV, no obstante, faltan aspectos por considerar para consolidar el enfoque. Por ejemplo, Broadband Forum al centrarse en mostrar los beneficios de NERG y especificar algunos requisitos para conseguirlos, omite la descripción detallada sobre cómo agregar nuevas funciones de red al vG. Si bien el TR-345 tiene la intención de soportar la arquitectura NERG, el direccionamiento de tráfico con base en una 5-tupla o en la aplicación (L7) que realiza la función de clasificación del MS-BNG, no representa un mecanismo preciso que permita identificar flujos de tráfico específicos de la red residencial y, por lo tanto, no se proporcionaría la capacidad plena de satisfacer los requerimientos de un tipo de tráfico determinado. CORD va más allá de las propuestas de Broadband Forum y presenta una plataforma unificada con base en los principios del "Cloud Networking". Sin embargo, R-CORD en su propuesta, no considera la creación de SFCs personalizadas por flujo de tráfico para una red residencial dada. Por otra parte, CORD no termina de definir el tipo de dispositivo a colocar en casa del usuario. Si bien en la descripción del vSG se propone reducir el RGW o CPE a un "baremetal switch", en la descripción de la OLT virtual (vOLT) ${ }^{34}$ se indica que el sistema extremo a extremo propuesto incluye un CPE controlado por OpenFlow. No obstante, no existe una aplicación de ONOS que se encargue de controlar dicho CPE. La aplicación vOLT solamente se encarga de controlar la OLT compatible con OpenFlow.

Si bien es cierto que NFV aprovecha la capacidad de las tecnologías en la Nube para proporcionar dinámicamente diferentes tipos de servicios con determinados requisitos computacionales, este

\footnotetext{
${ }^{32}$ https://opencord.org/

${ }^{33} \mathrm{https}$ ://wiki.opencord.org/pages/viewpage.action?pageId=1278090

${ }^{34} \mathrm{https}$ ://wiki.opencord.org/pages/viewpage. action?pageId=1278086
} 
paradigma se centra en proporcionar una réplica virtualizada de un dispositivo de red implementado con hardware específico y, por lo tanto, la propuesta de $\mathrm{vRGW}$ al colocar un dispositivo L2 restringe las capacidades de direccionamiento requeridas para controlar con precisión flujos de tráfico. Por ejemplo, resulta más eficaz filtrar tráfico no deseado localmente antes que realizarlo en el vRGW o podría presentarse la necesidad de asignar una prioridad a un determinado tipo de tráfico (Differentiated Services Code Point, DSCP) antes de que ingrese al vRGW. Por otra parte, aunque el enfoque de vRGW en principio permite agregar diferentes VNFs las cuales podrían estar destinadas a abordar temas relacionados a la Facilidad de Gestión, Visibilidad, QoS/QoE o Seguridad, no se garantiza una participación activa y total del usuario residencial en las tareas de gestión de sus propias redes. Así, se podría decir que la virtualización del RGW aborda, en parte, los problemas asociados a las redes residenciales como son la limitada usabilidad, alto grado de heterogeneidad del entorno y limitada flexibilidad para mejorar el servicio de conectividad de red provisto.

\subsubsection{Redes Definidas por Software (SDN)}

La aplicabilidad de los principios de SDN en entornos residenciales representa otra tendencia importante. Esta sección analiza las diferentes propuestas basadas en SDN considerando adicionalmente las diferentes configuraciones posibles relacionadas con la ubicación de los planos de control y datos, ya que los mismos se encuentran desacoplados y, la estrategia definida para abordar los requisitos relacionados a la Facilidad de Gestión, Visibilidad, QoS/QoE y Seguridad.

Yiakoumis et al. [62] y Gharakheili et al. [63] despliegan la capa de infraestructura SDN en el borde del ISP. Ambas propuestas permiten a los usuarios residenciales expresar sus propósitos o solicitudes a través de un "agente". Estos propósitos se traducen posteriormente a una semántica de red de bajo nivel y se entregan a un "agente de ISP" para modificar la configuración del nodo de borde. Ambos enfoques implementan las capas restantes en las instalaciones del ISP y pueden considerarse centradas en el usuario. Si bien las propuestas se enfocan en los requisitos de QoS y facilidad de gestión, Yiakoumis et al. no definen una estrategia que les permita abordar el resto de requisitos como lo hacen Gharakheili et al. al definir un enfoque basado en APIs. En este enfoque, desde un portal web, se acceden a dos APIs principales para modificar la configuración del dispositivo de borde. El enfoque es acertado, sin embargo, falta definir un marco o arquitectura que generalice la forma en la cual se ofrecen servicios desde la capa de control y el acceso a los mismos de una manera estándar, independientemente de la tecnología de controlador SDN subyacente utilizada.

Pediaditakis et al. [64] y Mortier et al. [65] proponen un RGW basado en Linux compuesto por un Open vSwitch $\left(\mathrm{OVS}^{35}\right)$ controlado por OpenFlow y un controlador NOX. En esta configuración, tanto el plano de datos como el de control están co-ubicados en el mismo dispositivo, proporcionando un RGW basado en SDN "todo en uno". Pediaditakis et al. proporcionan una aplicación para iPad encargada de guiar al usuario en la definición de políticas para gestionar el RGW. Un motor de políticas recibe la representación textual de la política desde la aplicación y la traduce a una descripción de tarea la cual se entrega al entorno de tiempo de ejecución para modificar la configuración del RGW. El entorno de tiempo de ejecución incorpora un servicio de configuración que permite representar la configuración general de la red como una colección de descripciones de tareas independientes de la plataforma y un gestor de monitoreo para almacenar información de uso y estado de la red. La propuesta permite abordar los requisitos desde la perspectiva del usuario, así como lo incluye activamente en las tareas de gestión.

Ciertamente, las políticas que se pueden crear dependen de las tareas que se proporcionan en el servicio de configuración. Si bien la arquitectura propuesta considera que se pueden agregar más tareas, es complejo que una única aplicación haga uso de todas ellas de una manera eficiente para ofrecer una amplia variedad de funcionalidades de gestión considerando los requisitos de usuario. Mortier et al. proponen que el controlador NOX exporte una interfaz de servicios web para controlar módulos que implementan un servidor DHCP personalizado, controlan el envío y la asociación inalámbrica a través de filtrado e interceptan las búsquedas de DNS con el objetivo de proporcionar un control por flujo más preciso. Los autores indican que los servicios proporcionados por el API permiten crear interfaces para la gestión de la red orientada a usuarios no expertos, mostrando un ejemplo de un panel de invitados que

\footnotetext{
${ }^{35} \mathrm{http}$ ://openvswitch.org/
} 
proporciona un punto de control central y una vista de la red. La arquitectura propuesta no considera extender las capacidades de gestión del API y dadas las reducidas opciones que se incorporan, no se garantiza que los requisitos de usuario se cumplan satisfactoriamente. Por otra parte, al implementar el plano de datos y control en el mismo dispositivo, ambos enfoques proporcionan una arquitectura cerrada que restringe el desempeño de los servicios que se ejecutan debido a los limitados recursos computacionales del hardware.

Otra configuración es presentada por Boussard et al. [66] y Gharakheili et al. [67]. En las instalaciones del usuario, la capa de infraestructura se implementa utilizando un RGW basado en SDN y las capas restantes se implementan en la infraestructura del ISP para proporcionar un entorno de gestión "basado en la Nube". Esta configuración aprovecha las capacidades de la infraestructura del ISP para superar las limitaciones de un RGW basado en SDN "todo en uno", como la disponibilidad limitada de recursos computacionales, los complejos procesos de actualización de software y hardware y la gestión remota. Boussard et al. proponen la creación de LANs definidas por software (SD-LANs) que representan redes virtuales/personales que incluyen diferentes dispositivos conectados y posibles servicios adicionales y funcionalidades del ISP o de terceros. Un controlador, denominado como "Majord'Home" abstrae los dispositivos u objetos conectados (CO) en la red residencial en objetos virtuales (VO) con el fin de proporcionar un control programable de ellos a través de una API común. El usuario final puede interactuar con Majord'Home a través de un cliente Android el cual proporciona una interfaz de usuario simple que permite la consulta de los diferentes VO pertenecientes a un controlador local o remoto para la creación de una SD-LAN.

Gharakheili et al. introducen el concepto de Proveedor de Gestión de Servicios (SMP) encargado de ejercer el control de configuración sobre el RGW en representación del usuario. SMP proporciona interfaces de personalización (portales web/aplicaciones) a los usuarios, traduciéndolas en operaciones de nivel de red invocadas mediante APIs. El API propuesto define tres categorías de interacciones: aplicaciones que emiten solicitudes para modificar el estado de la red, consultas para sondear el estado de la red y sugerencias para ayudar a mejorar el rendimiento. El SMP puede construir potentes servicios de valor agregado al componer estas APIs de red simples expuestas por el controlador. Como se sigue un enfoque "over-the-top", diferentes partes interesadas (proveedor de ISP o RGW) pueden implementar las capacidades de SMP en diferentes ubicaciones (Nube privada o Nube de terceros). Si bien ambas propuestas optan por incluir al usuario en las tareas de gestión y abordan la mayoría de sus requisitos, la apertura está restringida en ambas propuestas debido a que los marcos o arquitecturas no fueron concebidos como entornos de desarrollo.

La especificación de APIs estándar es necesaria para desarrollar y agregar fácilmente nuevas aplicaciones de gestión a cargo de mejorar diferentes aspectos de las redes residenciales, así como definir un conjunto básico de servicios a nivel del controlador que permitan componer servicios con un nivel mayor de abstracción. Del mismo modo, un enfoque independiente del controlador SDN podría adoptarse para suprimir la dependencia de una tecnología específica. Aunque la ubicación del plano de control en las instalaciones del ISP es más adecuada en comparación con un RGW basado en SDN "todo en uno", se debe explotar de mejor manera la flexibilidad y escalabilidad inherentes de las tecnologías en la Nube y NFV. Para ello, se requiere una arquitectura que defina la forma en la cual las capas lógicas de SDN serán implementadas en la infraestructura de computación en la Nube del ISP con el fin de cumplir con los requisitos de la solución basada en SDN.

A diferencia de permitir a los usuarios gestionar sus propias redes, se propone un enfoque para delegar la gestión de la red residencial a expertos externos o sistemas autónomos. Adicionalmente, existen propuestas que se especializan en abordar un determinado requisito del usuario. Por ejemplo, Kim y Feamster [68] y Calvert et al. [69] contribuyen a mejorar el requisito de Visibilidad utilizando un RGW basado en SDN "todo en uno". Kim y Feamster señalan que las mediciones de red pueden usarse para determinar si el rendimiento de la red residencial cumple con las expectativas del usuario. Calvert et al. proponen un sistema Registrador de Datos de Red Residencial que recopila datos de la red y proporciona a terceros esta información para crear nuevas aplicaciones a cargo de ayudar en la solución de problemas.

Con respecto a las mejoras en Seguridad, Feamster [70] propone externalizar la gestión de la seguridad. Se utiliza un RGW basado en SDN para recopilar estadísticas sobre la actividad de la red. Las 
estadísticas se envían a un controlador externo a cargo de realizar una inferencia distribuida para determinar el tráfico no deseado como botnets o malware y para filtrar el spam. Considerando que los requisitos de los usuarios son altamente heterogéneos, un enfoque basado en la externalización de la gestión debe enfrentar el desafío de cumplir con cada uno de ellos. Además, la adición de servicios de terceros representa un pago adicional que será cubierto, usualmente, por los usuarios finales.

Luo et al. [71] proponen un mecanismo para mitigar ataques de múltiples fases en Redes Residenciales Definidas por Software (SDHN). La detección de un evento en la red activa un proceso de evaluación de la seguridad basado en evidencia que utiliza un modelo de grafo de ataque e inferencia posterior. Con base en el resultado de la evaluación, el proceso de selección de contramedidas determina un plan de mitigación de ataque que indica al controlador SDN cómo instalar instancias de VNF en los nodos de red seleccionados. Aunque resulta de gran utilidad que los dispositivos de usuario final sean compatibles con las tecnologías SDN y NFV de acuerdo con el enfoque propuesto, los autores proporcionan una arquitectura de alto nivel, omitiendo detalles importantes que permitan comprender de mejor manera como se proporcionan todas las funcionalidades que se indican en la propuesta.

Con respecto a las mejoras en $Q o S / Q o E$, Jang et al. [72] proponen un marco de asignación de ancho de banda consciente de la QoS para el hogar inteligente basado en SDN considerando dispositivos M2M (machine to machine) y dispositivos no M2M. Para este fin, se propone modificar el identificador de clase de QoS (QCI) propuesto por el 3GPP LTE para adaptarlo a los servicios del hogar inteligente y se propone un algoritmo de planificación que considera la equidad, el retraso y la prioridad. Un RGW habilitado para OpenFlow es desplegado en las instalaciones del usuario mientras que el ISP mantiene un "SDN Smart Home Cloud" destinado a crear una red virtual de hogar dedicada (Home VN) por cada hogar inteligente. Si bien se considera la infraestructura en la Nube del ISP, la propuesta es agnóstica del paradigma NFV. Además, un único controlador para varios hogares inteligentes no representa una solución adecuada considerando que un eventual fallo en el controlador SDN significaría que todos los hogares inteligentes perderían el servicio de asignación de ancho de banda.

Por otra parte, Abuteir et al. [73] proponen NAVS (Network Assisted Video Streaming) para realizar un conformado de tráfico dinámico para diferentes flujos de video en función de la política definida (por el usuario o pre-definidas por el sistema), el número de flujos de video, los bitrates de video disponibles y el ancho de banda del acceso a Internet. Para este fin, NAVS integra diferentes módulos para definir políticas, monitorizar el tráfico, analizar el MPD (Media Presentation Description), optimizar la QoE y conformar el tráfico. Los autores afirman que la asignación de ancho de banda para cada flujo de video se realiza en tiempo real. No obstante, no especifican la ubicación del controlador ni la tecnología de controlador utilizada lo cual afecta directamente a la capacidad de reacción de NAVS. Adicionalmente, los autores deberían considerar que el eventual uso de control "en banda" en SDN también podría afectar la capacidad de reacción, sobre todo en casos de congestión.

Si bien un RGW basado en SDN proporciona mejores condiciones en comparación con un RGW tradicional para optimizar los procesos de gestión y enriquecer el servicio de conectividad de red provisto, este enfoque se podría beneficiar de propuestas adicionales relacionadas a la red de acceso y al segmento inalámbrico de la red residencial con el propósito de proporcionar una solución integral. Por ejemplo, Rükert et al. [74] proponen que tanto el RGW, el nodo de acceso, los conmutadores de la red de agregación y el BRAS (Broadband Remote Access Server) sean compatibles con SDN. Este enfoque puede considerarse un caso ideal y, como argumentan los autores, la configuración de los flujos de tráfico puede ser más dinámica, optimizando el rendimiento de la red, especialmente para los servicios que consumen un gran ancho de banda.

En una aproximación similar, Gharakheili et al. [75] proponen que el nodo de acceso se conecte a un conmutador Ethernet SDN que a su vez se conecta a la red backhaul del ISP para proporcionar acceso a Internet. El conmutador SDN está controlado por un controlador SDN que se encuentra dentro de la red del ISP y expone APIs que son utilizadas tanto por el usuario final como por los proveedores de servicios de contenido (CSP) para gestionar de mejor manera el ancho de banda limitado del acceso a Internet. Con respecto a una Red de Acceso Definida por Software (SDAN), Kerpez et al. [76] indican que esta propuesta tiene como objetivo abstraer toda la red de acceso por medio de una interfaz unificada y 
común para gestión y control que permite la creación ágil de nuevos servicios adaptados a las necesidades del usuario.

Considerando que los medios de transmisión ópticos se están convirtiendo en la opción preferida para las redes de acceso, se requiere que el nodo de acceso u OLT (Optical Line Terminal) sea compatible con los principios de SDN. En la actualidad, OLTs basados en SDN que implementen nativamente OpenFlow no están usualmente disponibles. Para solventar este inconveniente, Thyagaturu et al. [77] indican que se utiliza una capa de abstracción de hardware que permita convertir OLTs tradicionales en dispositivos controlados por OpenFlow. Diferentes propuestas están alineadas con este enfoque. Por ejemplo, Clegg et al. [78] presentan una GPON (Gigabit-capable Passive Optical Network) habilitada para OpenFlow. La capa de abstracción de hardware presenta al controlador externo OpenFlow un único conmutador distribuido con varios puertos, uno para el OLT y los puertos restantes para cada ONT (Optical Network Terminal). Lee et al. [79] presentan una propuesta similar en la cual un agente OpenFlow embebido reside dentro de la OLT. El agente interactúa con el controlador externo OpenFlow y el módulo GPON OMCI (ONT Management and Control Interface), transformando todo el sistema GPON en un conmutador virtual.

Gallo et al. [80] proponen aplicar los principios de SDN para abordar las complejidades de las redes residenciales inalámbricas. Los autores introducen la arquitectura "SDN@home" en la cual el plano de control de diversas redes domésticas inalámbricas (por ejemplo, entretenimiento, automatización o cuidado de la salud) está separado del plano de datos y, el RGW con soporte para múltiples tecnologías, actúa como un controlador central. Este enfoque permite que dispositivos inalámbricos de propósito general sean programados al instante mediante la instalación de programas de radio en función de diferentes escenarios de aplicación y condiciones cambiantes que afectan a la red residencial.

\subsection{Conclusiones}

De la revisión del estado del arte realizada en este capítulo, se concluye que el modelo actual de gestión y provisión de servicios para redes residenciales es poco flexible, no responde a las necesidades y preferencias cambiantes y heterogéneas de los usuarios y limita la creación de nuevos modelos de negocio para el ISP. En este contexto, un nuevo paradigma de gestión para la red residencial con base en el enfoque centrado en el usuario representa una solución acertada no solamente para mejorar la usabilidad y abordar el tema de la alta heterogeneidad del entorno, sino también para enriquecer las funcionalidades que proporciona la red residencial y ampliar el catálogo de servicios del ISP. Diferentes propuestas adoptan un enfoque similar y plantean el uso de las prominentes características que ofrecen las tecnologías NFV y SDN para conseguirlo.

De las propuestas analizadas se reconoce una tendencia hacia la virtualización del RGW mayormente soportado por el paradigma NFV, siendo la arquitectura NERG de Broadband Forum y el proyecto CORD los ejemplos más sobresalientes. Por otra parte, existen propuestas que abogan por la implementación de un RGW basado en SDN para aprovechar su programabilidad precisa en la creación de mejores modelos de gestión en los que el usuario residencial puede desempeñar el papel de gestor de red. Sin embargo, el estado del arte muestra la falta de una arquitectura de gestión prolijamente definida que satisfactoriamente aborde todas las necesidades desde la perspectiva del usuario residencial. Aunque la mayoría de las propuestas ofrecen resultados con respecto a la Facilidad de Gestión o QoS, sus arquitecturas no se explican en detalle $\mathrm{y}$, como consecuencia, no es posible determinar cómo las propuestas cumplen con los requisitos de otros aspectos de mejora como Visibilidad o Seguridad.

Si bien un enfoque basado en la Nube permite que el plano de control de un RGW basado en SDN sea desplegado en las instalaciones del ISP, el enfoque podría utilizar los principios de NFV con el objetivo de maximizar el uso del control preciso del tráfico que proporciona una red residencial basada en SDN. No obstante, las propuestas analizadas no consideran la opción de combinar los paradigmas SDN y NFV en una única solución integral de gestión. Así, una solución híbrida representa un enfoque conveniente que permite explotar los beneficios de NFV para crear SFCs personalizadas encargadas de procesar un determinado tipo de tráfico $\mathrm{y}$, de SDN, para dirigir el tráfico que requiere un procesamiento en particular a la SFC adecuada. De esta manera, considerando que la red residencial proporciona servicios de 
conectividad como se explicó en la sección 2.1.2, el enfoque NFV-SDN permitiría ampliar y mejorar estos servicios en comparación con el RGW tradicional. Sin embargo, para proporcionar una solución de gestión y despliegue de esta naturaleza, es importante considerar los siguientes requisitos:

- El rol activo de los usuarios en las tareas de gestión requiere el diseño de un marco o arquitectura de gestión específico y definido cuidadosamente para redes residenciales el cual organice y estandarice cómo las diferentes capas de software o componentes funcionales controlan el RGW basado en SDN e interaccionan con el componente de Gestión y Orquestación (MANO) de NFV para desplegar VNFs dinámicamente.

- Las tareas de gestión se pueden distribuir en diferentes herramientas o aplicaciones considerando el alto grado de heterogeneidad que presenta el entorno residencial y lo complejo que resulta el desarrollo de una única herramienta de gestión que satisfaga las diferentes necesidades de los usuarios. Además, este enfoque permitiría desarrollar aplicaciones especializadas en mejorar un determinado aspecto de la red residencial como la Facilidad de Gestión, Visibilidad, QoS/QoE o Seguridad.

Por otra parte, también es importante implementar un enfoque interdisciplinario el cual permita utilizar, por ejemplo, técnicas de interacción hombre-computadora (HCI) para mantener uniformidad en el desarrollo de interfaces gráficas y procedimientos de gestión adaptados a las habilidades y necesidades de los usuarios.

- La arquitectura de gestión requiere implementar el principio de apertura para alentar a la comunidad de desarrolladores a participar en la creación de aplicaciones de gestión para entornos residenciales. Para cumplir con este objetivo, los detalles de configuración de red de bajo nivel deben ocultarse mediante la provisión de APIs con un nivel alto de abstracción. Así, en función de las necesidades del usuario, los desarrolladores son capaces de implementar un enfoque de composición de servicios para crear nuevas aplicaciones con funcionalidades avanzadas. Por otra parte, debido a la heterogeneidad de los controladores SDN, la interoperabilidad/portabilidad de las aplicaciones SDN no se puede implementar y los desarrolladores están vinculados a una tecnología de controlador específica lo cual representa una desventaja significativa que pone en peligro el principio de apertura de SDN. Para evitar este inconveniente, la solución de gestión debería considerar la implementación de un enfoque independiente del controlador SDN.

- Un enfoque de gestión basado en la Nube implica implementar el plano de control SDN y las diferentes VNFs en la infraestructura de NFV (NFVI) desplegada en las instalaciones del ISP. En este sentido, se requiere un diseño específico del espacio virtual asignado a cada red residencial dentro de la NFVI considerando las características específicas de los entornos residenciales, los requisitos de la solución basada en SDN y la adición de diferentes VNFs para mejorar el servicio de conectividad de red que se proporciona. Adicionalmente es importante definir una ubicación adecuada para el espacio virtual de la red residencial con el objetivo de no afectar la capacidad de reacción del controlador SDN ni incrementar significativamente la latencia de los servicios que se consumen debido a las tecnologías de virtualización utilizadas. Así, un enfoque híbrido como el que se utiliza en varias arquitecturas vCPE empresariales podría ser considerado.

- El control preciso del tráfico que proporciona un RGW basado en SDN conjuntamente con la posibilidad de crear SFCs específicas destinadas a procesar un determinado tipo de tráfico mejoran notablemente el servicio de conectividad que proporciona la red residencial. No obstante, como se menciona en el estado del arte, es importante considerar las ventajas que ofrecen las Redes de Acceso Definidas por Software (SDAN) en la creación ágil de nuevos servicios adaptados a las necesidades del usuario. De esta manera, la solución de gestión y despliegue podría incluir los principios de SDAN para complementar el servicio de conectividad que proporciona una red residencial basada en SDN y NFV.

De esta manera, en los siguientes capítulos se procederá a abordar los diferentes requisitos enumerados previamente mediante la propuesta de una nueva arquitectura de gestión para la red residencial. Para este fin, se hará uso extensivo de los paradigmas SDN y NFV como tecnologías habilitantes conjuntamente con el enfoque centrado en el usuario. 


\section{Capítulo 3}

\section{Arquitectura de Gestión Centrada en el Usuario para Redes Residenciales}

De acuerdo con lo analizado en la sección 2.1.3, gestionar una red residencial significa configurar las funcionalidades proporcionadas por el $\mathrm{RGW}$, lo cual generalmente requiere un cierto nivel de experiencia técnica de la que carecen la mayoría de los usuarios residenciales. Los proveedores de servicios de Internet (ISPs) a veces abordan este problema de usabilidad gestionando el RGW desde una ubicación central y ofreciendo la opción de configurar funciones simples como la contraseña de la WLAN a través de una aplicación basada en la web. En este capítulo, se propone una nueva arquitectura de gestión que, en primer lugar, permite utilizar el enfoque centrado en el usuario para abordar los problemas de la red residencial identificados en el capítulo del estado del arte como son la limitada usabilidad de la red, la alta heterogeneidad existente y la limitada flexibilidad para agregar nuevas funciones que permitan enriquecer el servicio de conectividad de red que se proporciona. Para conseguir la centralidad en el usuario, se propone crear un entorno de gestión basado en aplicaciones las cuales están adaptadas a las habilidades de un usuario promedio. Así, el usuario es capaz de gestionar su propia red y personalizarla según sus necesidades. En segundo lugar, la arquitectura facilita el desarrollo de las aplicaciones encargadas de gestionar la red residencial mediante la provisión de diferentes servicios encargados de simplificar el proceso de configuración y monitoreo de red de bajo nivel. La arquitectura propuesta aprovecha el control preciso del tráfico de red y la conveniencia para comunicar eventos de red que proporciona SDN, así como el enfoque de virtualización del RGW (vRGW) para externalizar funciones de red tradicionales desde el RGW a una infraestructura en la Nube administrada por el ISP.

\subsection{Introducción}

De la revisión del estado del arte realizada en la sección 2.1 se concluye que el modelo de red utilizado para implementar y gestionar una red residencial no ha evolucionado sustancialmente desde su concepción. A pesar de que la provisión de ancho de banda ha aumentado, llegando a cientos de Mbps hoy en día, la heterogeneidad de las redes residenciales y los requisitos divergentes de los diferentes servicios multimedia que se ejecutan en las redes residenciales aún son temas por resolver. Proporcionar a los usuarios residenciales la capacidad de personalizar sus redes permitiría mejorar la usabilidad o capacidad de gestión de la red. Sin embargo, esto representa un verdadero desafío porque las redes residenciales han adoptado un modelo de gestión al estilo de Internet el cual requiere un cierto nivel de conocimientos de redes y, por lo tanto, no es adecuado para usuarios no expertos en tecnología. Para aliviar esta situación, se han presentado varias propuestas para proporcionar a los usuarios residenciales una participación activa en las tareas de gestión de la red residencial, tal y como se mostró en la sección 2.4.2. Sin embargo, estas propuestas, no proporcionan un modelo efectivo para involucrar realmente a los usuarios residenciales en la gestión de sus propias redes. 
En este capítulo se presenta una propuesta para abordar la gestión de la red residencial considerando un enfoque centrado en el usuario que aprovecha la experiencia y la familiaridad con el uso de aplicaciones móviles que poseen la mayoría de usuarios. De esta manera, se presenta la aplicación para la Gestión de la Red Residencial, denominada como "aplicación RENEMA", a través de la cual el usuario es capaz de ejercer las tareas de gestión de su propia red. Se aborda de forma integral el tema de la alta heterogeneidad que presenta el entorno residencial al distribuir las tareas de gestión entre diferentes aplicaciones, cada una de ellas especializada en tratar uno de los cuatro aspectos de mejora definidos en la sección 2.1.4 (Facilidad de Gestión, Visibilidad, QoS/QoE y Seguridad). Por otra parte, la arquitectura tiene como objetivo simplificar el desarrollo de nuevas aplicaciones al proporcionar diferentes Servicios de Red Residencial (RENESE) a cargo de ocultar los detalles de configuración y monitoreo de red de bajo nivel.

El paradigma SDN se usa para aprovechar dos aspectos. En primer lugar, la programabilidad precisa que presenta el plano de datos. En segundo lugar, la forma natural para comunicar eventos de red que se proporciona. Adicionalmente, considerando el criterio de Rothenberg et al. [81] al decir que la programabilidad de red está respaldada por la industria centrada en el software, este enfoque se beneficiará de las tecnologías ya existentes que facilitan la creación de sofisticadas herramientas de gestión que pueden implementarse y actualizarse fácilmente. Sin embargo, la arquitectura de referencia SDN en sí misma [22] no proporciona un enfoque centrado en el usuario. En este capítulo, se definen varios principios para guiar el diseño de una nueva arquitectura que proporciona las bases para impulsar la evolución de las redes residenciales hacia este nuevo paradigma de gestión y provisión de servicios. Como resultado, se agregan dos capas sobre la parte superior de la arquitectura de referencia SDN las cuales se encargan de proporcionar a los usuarios residenciales una arquitectura de gestión SDN centrada en el usuario.

El enfoque SDN implica reemplazar el RGW tradicional por uno compatible con esta tecnología y, en consecuencia, también implica seleccionar una ubicación física para implementar las capas de software restantes, así como externalizar las funciones de red tradicionales como encaminamiento o NAT. Teniendo en cuenta los recursos computacionales limitados de los RGWs tradicionales y el advenimiento del paradigma NFV, se prevé un entorno de gestión basado en la Nube, similar al enfoque de vRGW. En este escenario, se puede implementar un Dominio de Gestión y Redes virtualizado (vMANDO) para cada red residencial en la infraestructura en la Nube del ISP. Cada vMANDO contendrá los diferentes componentes de software de la arquitectura de gestión y las funciones de red virtualizadas (VNF) que son agregadas para enriquecer el servicio de conectividad que proporciona la red residencial.

Este nuevo paradigma mejora significativamente la flexibilidad y puede ser considerado por las Organizaciones de Desarrollo de Estándares (SDOs) para definir nuevos modelos de red para redes residenciales, así como también nuevos estándares para SDN y NFV. Por otra parte, vMANDO representa un medio para alcanzar un alto grado de automatización en los procesos de despliegue, gestión y mantenimiento de redes residenciales lo cual resulta de especial interés para los ISPs.

\subsection{Diseño de la Arquitectura de Gestión Centrada en el Usuario}

\subsubsection{Aplicación para la Gestión de la Red Residencial}

La usabilidad [82] de un sistema es el objetivo principal que la centralidad en el usuario trata de conseguir. La provisión de un sistema de gestión a medida permitiría a los usuarios residenciales tomar el control de sus redes y, por lo tanto, incrementar el nivel de usabilidad. Sin embargo, para conseguir esta centralidad en el usuario, se requiere un mecanismo efectivo para involucrar a los usuarios en las tareas de gestión. Siguiendo la propuesta de Yiakoumis et al. [62], un "agente de usuario" permite capturar los propósitos o solicitudes del usuario para traducirlas posteriormente a la semántica de red. Este enfoque se complementa con el argumento de que las habilidades técnicas comunes de los usuarios residenciales deben considerarse en la definición de dicho "agente de usuario".

Considerando la familiaridad de la mayoría de los usuarios con la utilización de aplicaciones de Internet, ya sea en computadores o dispositivos móviles, se propone que una aplicación para la Gestión de la Red 
Residencial, denominada como aplicación RENEMA (RENEMA app), cumpla con las funciones de "agente de usuario". Esta aplicación tiene como objetivo integrar exitosamente al usuario en el sistema de gestión de la red residencial como se muestra en la Figura 3.1. El diseño cuidadoso de un conjunto de aplicaciones permitiría abordar el tema de la alta heterogeneidad del entorno residencial y mejorar cualquiera de los aspectos relacionados con las redes residenciales presentados en la sección 2.1.4 ya que el usuario escoge que aplicación utilizará con base en sus necesidades. Por ejemplo, se puede desarrollar una aplicación de control parental para gestionar fácilmente el acceso a Internet de los usuarios y sus dispositivos con base en horarios o incluso contenido web. Además, esta aplicación podría ayudar a solicitar niveles de QoS específicos cuando se va a reproducir contenido multimedia.

Para alcanzar sus objetivos, las aplicaciones de gestión imponen algunos requisitos en el RGW, tales como:

- La capacidad para especificar de forma precisa el recorrido de paquetes a través de la red con el objetivo de ejercer un control de grano fino sobre el tráfico del usuario. Aunque algunos protocolos como el Protocolo de Configuración de Red (NETCONF) [9] y el Protocolo de Gestión WAN CPE (CWMP/TR-069) [10] brindan opciones para configurar y monitorear el RGW, ninguno de ellos está orientado a la configuración del plano de datos.

- Un mecanismo de retroalimentación para informar sobre el estado de la red. Para un mejor control, las aplicaciones deben estar informadas sobre cualquier cambio en el estado de la red mediante eventos de red. Una vez que las aplicaciones procesan estos eventos, el comportamiento de la red puede modificarse a un estado específico con base en las decisiones del usuario.

- Un conjunto más completo de opciones de configuración relacionadas con QoS y monitoreo. Debido al alto nivel de personalización que ofrece un enfoque centrado en el usuario, se debe proporcionar un mecanismo detallado para la definición de políticas para controlar los recursos de red como el ancho de banda. De manera similar, las tareas de monitoreo deben proporcionar estadísticas con diferentes niveles de agregación para enriquecer la visibilidad de la red.

- Un alto nivel de capacidad de respuesta o de reacción de la red. Considerando que las aplicaciones son interactivas por definición, cualquier modificación llevada a cabo por el usuario debe aplicarse inmediatamente para garantizar una experiencia de usuario impecable.

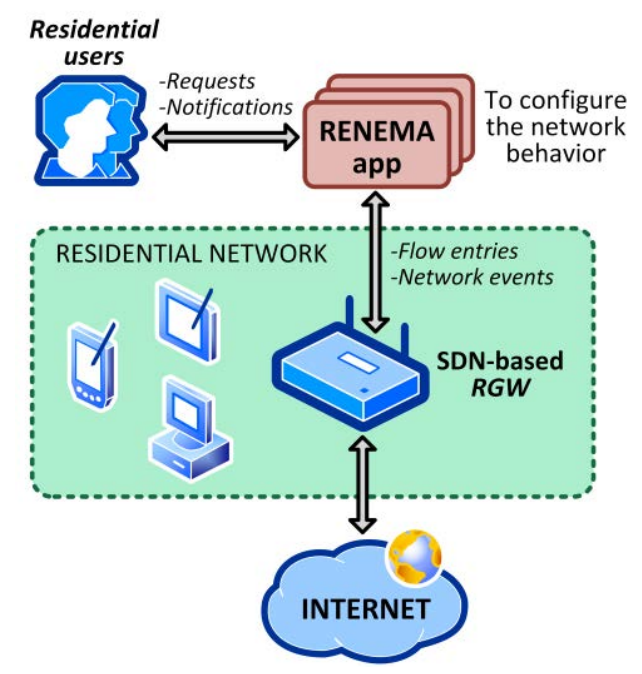

Figura 3.1 Enfoque de alto nivel centrado en el usuario. Las aplicaciones RENEMA brindan a los usuarios residenciales la capacidad de configurar diferentes parámetros de la red.

\subsubsection{Principios de Diseño de la Arquitectura}

El paradigma de redes tradicional no ofrece la flexibilidad y la programabilidad necesarias para cumplir con los requisitos de red planteados por las aplicaciones RENEMA. Por el contrario, las tecnologías SDN son idóneas para este propósito. La separación del plano de control del plano de datos propuesto 
por el paradigma SDN [21] presenta una oportunidad para implementar el control preciso del tráfico necesario en las redes residenciales. La programabilidad del plano de datos permite a las aplicaciones RENEMA modificar el comportamiento de la red de forma proactiva, en respuesta a una solicitud del usuario o, de forma reactiva, en respuesta a un evento de red. Del mismo modo, SDN proporciona un alto grado de capacidad de respuesta y un amplio conjunto de opciones para configurar políticas de QoS complejas y para monitorizar la red exhaustivamente.

Sin embargo, la arquitectura de referencia SDN es demasiado genérica [22]. La arquitectura describe a un alto nivel de abstracción los componentes arquitectónicos, puntos de referencia e interfaces. Los bloques funcionales internos del controlador SDN también se describen, aunque la interacción entre ellos no se especifica. Además, en lo que se refiere a la interfaz Northbound, la arquitectura no define protocolos, procedimientos, formatos de mensajes y semántica para intercambiar información de gestión. Aunque la arquitectura de referencia SDN proporciona pautas importantes, se requiere una definición y especificación adicionales para diseñar una arquitectura de gestión centrada en el usuario que se pueda aplicar al entorno de la red residencial. Con el fin de diseñar dicha arquitectura, a continuación se procede a definir un conjunto de principios con base en las conclusiones obtenidas de la revisión del estado del arte y considerando otras tecnologías complementarias como la computación en la Nube o nuevos conceptos como "Future Networks" presentado por la recomendación ITU-T Y.3001 [83]:

a. Red usable o gestionable. En la actualidad, las redes residenciales son difíciles de usar o gestionar debido a la falta de herramientas adecuadas. La provisión de un entorno de gestión eficaz, fácil de usar y simple permitiría a los usuarios gestionar sus propias redes sin problemas $\mathrm{y}$, por lo tanto, mejorar la usabilidad o capacidad de gestión.

b. Procedimientos de gestión comunes. Considerando que las actividades de gestión pueden llevarse a cabo de varias maneras, el objetivo es definir procedimientos comunes de gestión que se adapten a las destrezas técnicas de un usuario promedio. De forma similar, es importante definir guías para desarrollar interfaces gráficas para aplicaciones a fin de mantener un estilo común y una apariencia y sensación consistentes.

c. Independencia. Se definen tres niveles diferentes de independencia para la arquitectura. En primer lugar, las actividades de gestión deben llevarse a cabo desde diferentes dispositivos electrónicos (por ejemplo, smartphones o laptops), así como desde diferentes lugares (por ejemplo, casa o trabajo). En segundo lugar, considerando la amplia comunidad de desarrolladores, la arquitectura no puede estar atada a un lenguaje de programación específico o una plataforma de desarrollo. En tercer lugar, los diferentes componentes de la arquitectura podrían implementarse en diferentes lugares físicos en función de la infraestructura computacional disponible.

d. Arquitectura abierta. Se pueden desarrollar diferentes aplicaciones que permitan, en primer lugar, abordar el tema de la alta heterogeneidad del entorno residencial $\mathrm{y}$, en segundo lugar, mejorar determinados aspectos de la red residencial como la Facilidad de Gestión, Visibilidad, QoS/QoE o Seguridad. Por lo tanto, un enfoque de arquitectura abierta permitiría agregar nuevas aplicaciones RENEMA para proporcionar una amplia variedad de herramientas de gestión, así como para actualizarlas. Del mismo modo, debido a la naturaleza abierta de la arquitectura (no depende de ningún proveedor específico), cualquier desarrollador podría contribuir con el entorno de gestión.

e. Servicios de Red Residencial. Una tarea de gestión realizada por el usuario representa una interacción entre la aplicación RENEMA y el RGW basado en SDN. Para evitar que los desarrolladores realicen tareas de configuración o monitorización de red de bajo nivel, la arquitectura necesita proporcionar varios Servicios de Red Residencial (RENESEs). Además, se requiere un modelo de interacción eficaz y flexible entre servicios y aplicaciones para garantizar la adopción de la arquitectura en la comunidad de desarrolladores.

f. Integración de Computación en la Nube y NFV. Considerando el alto nivel de madurez alcanzado por las tecnologías en la Nube en la provisión de servicios más eficientes, flexibles y escalables, la arquitectura debería integrar estas valiosas características con el objetivo de proporcionar un enfoque de gestión basado en la Nube. Los recursos de la Nube del ISP no solo 
proporcionan las ventajas de un entorno virtualizado para desplegar los componentes de software de la arquitectura, sino que también ayudan a superar las restricciones que impone un dispositivo con recursos limitados como el que se despliega en las instalaciones del usuario. Por otra parte, desde la perspectiva del paradigma NFV, la infraestructura de computación en la Nube del ISP representa una NFVI que, conjuntamente con los componentes de Gestión y Orquestación, permitirían desplegar nuevos servicios con funcionalidades avanzadas empaquetados como Funciones de Red Virtualizadas destinadas a mejorar el servicio de conectividad que proporciona la red residencial.

Siguiendo estos principios, la nueva arquitectura concebida se representa en la Figura 3.2, en la cual, la aplicación RENEMA reside en la parte superior de la arquitectura base SDN para proporcionar un nuevo entorno de gestión centrado en el usuario para entornos residenciales. Las siguientes subsecciones describen cada capa, la definición y especificación de bloques funcionales complementarios, así como sus modelos de interacción correspondientes en detalle.

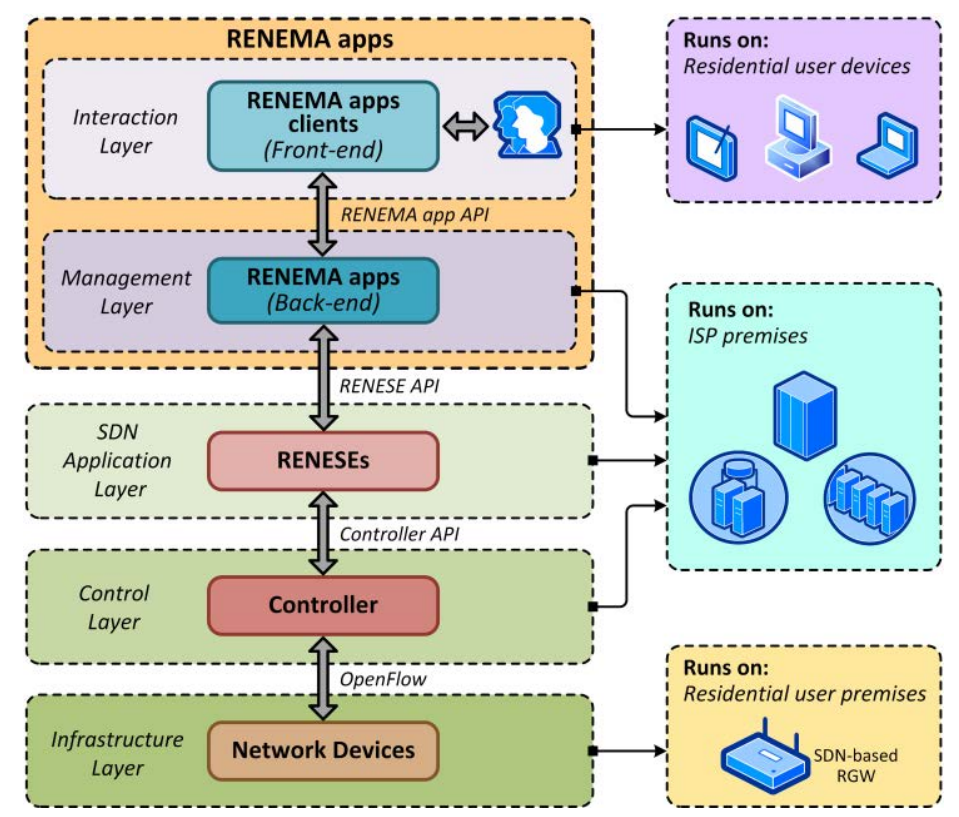

Figura 3.2 Arquitectura de gestión de referencia centrada en el usuario. La centralidad en el usuario se consigue al colocar las capas de Interacción y de Gestión en la parte superior de la arquitectura base SDN. Estas capas adicionales forman juntas una aplicación RENEMA. Nótese cómo diferentes capas se ejecutan en diferentes ubicaciones.

\subsubsection{La Capa de Interacción}

La funcionalidad de las aplicaciones RENEMA se divide en dos partes: el componente front-end que se ejecuta en la capa de Interacción y el componente back-end que se ejecuta en la capa de Gestión. Estas dos capas permiten que se utilice el enfoque centrado en el usuario para gestionar la red residencial. La capa de Interacción se puede implementar en diferentes dispositivos como smartphones, tablets o PCs y básicamente contiene la interfaz de usuario de la aplicación, denominado como cliente de la aplicación RENEMA (RENEMA app client) como se observa en la Figura 3.3. A través de este cliente, el usuario interactúa con el sistema para gestionar la red residencial y recibir notificaciones sobre el estado de la red. La capa de Interacción se comunica con la capa de Gestión por medio de un API basada en la tecnología REST, denominado como API de la aplicación RENEMA (RENEMA app API). REST representa la opción preferida para este tipo de interfaces debido a su simplicidad y desacoplamiento entre clientes y servidores.

Con el fin de proporcionar la centralidad en el usuario, el desarrollo de aplicaciones RENEMA debe estar alineado con los principios $a$ y $b$ definidos previamente. Así, es de máxima importancia considerar técnicas de interacción hombre-computadora (HCI) que permitan definir estándares para interfaces 
gráficas y procedimientos de gestión que cumplan con los requisitos de un usuario residencial promedio. De esta manera el usuario no será abrumado con aplicaciones de gestión inconsistentes y heterogéneas. Finalmente, la división de aplicaciones de gestión en dos capas unidas por un API REST, junto con la posibilidad de desplegar la capa de Interacción sobre diferentes dispositivos y sistemas operativos, hace que la arquitectura cumpla con el principio $c$, evitando que los usuarios residenciales estén atados a un RGW específico o fabricante para gestionar sus redes.

\subsubsection{La Capa de Gestión}

Esta capa representa la funcionalidad de back-end de la aplicación RENEMA, la cual se encarga de configurar el comportamiento de la red residencial (RGW basado en SDN). Según el principio $f$, esta capa de Gestión se despliega en las instalaciones del ISP con el objetivo de aprovechar los recursos de su infraestructura en la Nube (NFVI). Además, considerando el alto grado de heterogeneidad de este tipo de entorno, resulta conveniente distribuir la tarea de gestión de red entre diferentes aplicaciones, cada una proporcionando una funcionalidad específica a cargo de mejorar un aspecto determinado de la red residencial (sección 2.1.4). De esta manera, el usuario con base en sus habilidades y necesidades específicas es capaz de personalizar su sistema de gestión. Las aplicaciones RENEMA están disponibles para usuarios residenciales siguiendo el conocido modelo de tienda de aplicaciones que se utiliza principalmente en dispositivos móviles. Las nuevas aplicaciones se pueden agregar o actualizar fácilmente, proporcionando un entorno de gestión integral para redes residenciales como se establece en el principio $d$.

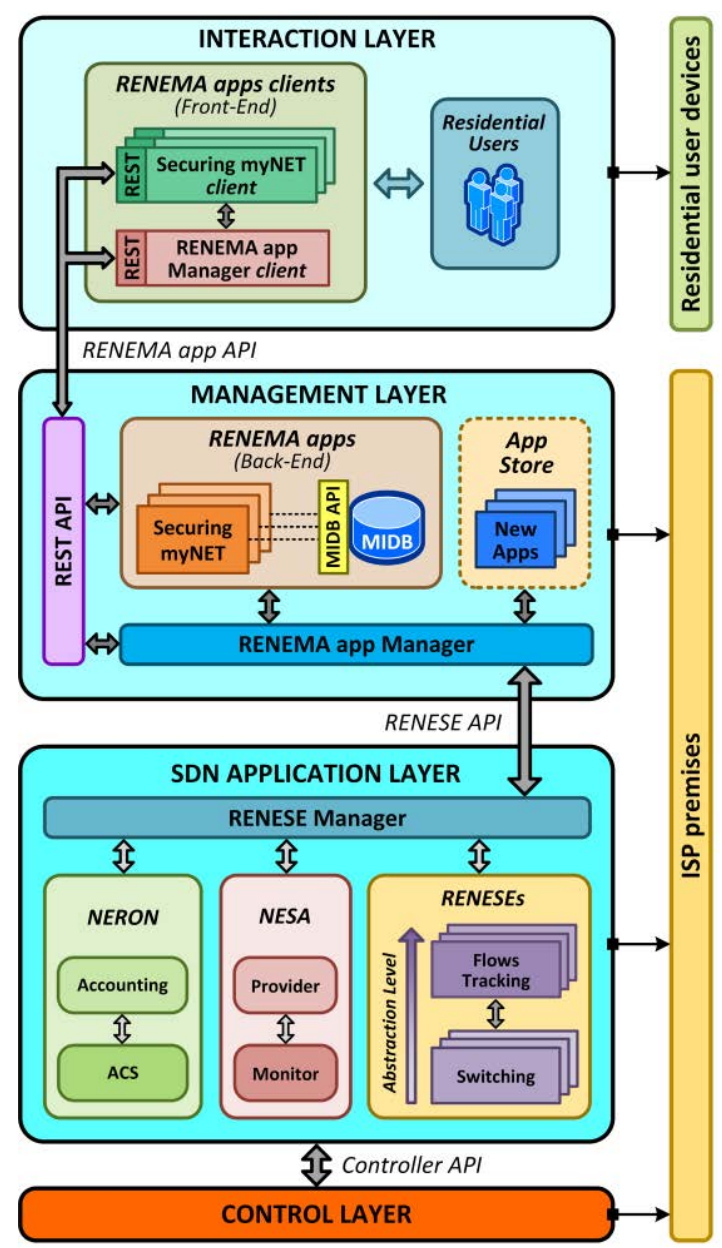

Figura 3.3 Bloques funcionales internos organizados en diferentes capas. Nótese cómo la comunicación entre capas se realiza a través de APIs específicas. La capa de Interacción se ejecuta en los dispositivos preferidos del usuario residencial mientras que el resto de capas de software se ejecutan en las instalaciones del ISP. 
Como se observa en la Figura 3.3, cada aplicación proporciona su propia API REST, la cual incluye la especificación de URIs (Uniform Resource Identifiers), métodos y argumentos de acuerdo con el conjunto de funciones de gestión que se proporcionará. En la sección 3.4.3, se incluye información detallada sobre el funcionamiento de las aplicaciones RENEMA mientras que, en el capítulo 5, se presenta un caso práctico de uso en el cual se describe con más detalle la estructura del API. Por otra parte, las funciones proporcionadas por las aplicaciones se basan en el uso de los Servicios de Red Residencial denominados como RENESEs. La capa de Aplicación SDN está a cargo de proporcionar diferentes RENESEs o servicios de forma general, con el objetivo de ocultar los detalles de configuración y monitoreo de red de bajo nivel a los desarrolladores de aplicaciones.

La existencia de múltiples aplicaciones en la capa de Gestión plantea la necesidad de definir el Gestor de aplicación RENEMA (RENEMA app Manager) a cargo de realizar las siguientes tareas:

- La provisión de un entorno apropiado para desplegar y ejecutar aplicaciones RENEMA. En este contexto, se abordan ciertos procesos como la instalación/desinstalación, la comunicación con la tienda de aplicaciones, la asignación de recursos, la monitorización, el mantenimiento, entre otros.

- La asignación de un identificador (Id) para cada aplicación el cual se utiliza para coordinar el intercambio de información de gestión entre las capas de Gestión y Aplicación SDN.

Como se observa en la Figura 3.3, el Gestor de aplicación RENEMA es, en sí mismo, otra aplicación, con su propio cliente ejecutándose en la capa Interacción para permitir a los usuarios residenciales gestionar y personalizar todo el entorno de gestión y coordinar las diversas aplicaciones. Finalmente, se agrega una Base de Datos de Información de Gestión (MIDB) en la capa de Gestión para proporcionar un almacenamiento común para las aplicaciones, lo que les permite almacenar y compartir información. El MIDB incluye un API para simplificar los procesos relacionados con la administración de la base de datos.

\subsubsection{La Capa de Aplicación SDN}

Para facilitar la implementación del enfoque centrado en el usuario y cumplir con los requisitos de las aplicaciones RENEMA, la capa de Aplicación SDN pone a disposición varios Servicios de Red Residencial (RENESEs), cada uno proporcionando un conjunto de funciones específicas o API con un determinado nivel de abstracción que oculta la complejidad de la configuración y monitorización de la red a la vez que permite componer servicios, simplificando el trabajo a los desarrolladores de acuerdo con el principio e. Siguiendo el mismo principio, se define un protocolo de nivel de aplicación, designado como protocolo RENESE, para especificar cómo los servicios que se ejecutan en esta capa interactúan entre sí y con las aplicaciones que se ejecutan en la capa de Gestión.

El conjunto de funciones que proporciona cada servicio se exporta utilizando el protocolo RENESE como se muestra en la Figura 3.3. Considerando que los usuarios residenciales tienen varios requisitos y las interfaces Northbound [84] no están estandarizadas, la arquitectura propuesta tiene como objetivo definir los servicios requeridos en esta capa para crear diferentes aplicaciones a cargo de mejorar la usabilidad de la red residencial y abordar el tema de la heterogeneidad presente. Además, la definición de un protocolo de nivel de aplicación permite especificar cómo se pueden ofrecer servicios a entidades dentro de la misma capa o a entidades externas que residen en capas superiores.

El protocolo RENESE define un único mensaje para intercambiar información de gestión como se muestra en la Figura 3.4. El mensaje contiene cuatro campos: dos para identificar al remitente (SenderId) y al receptor (ReceiverId) del mensaje y dos para indicar el servicio solicitado (Service) y sus respectivos argumentos (Arguments). El campo servicio representa el conjunto de funciones o API de cada RENESE. Se lo ha denominado como "servicio" para enfatizar que la capa de Aplicación SDN, de forma general, proporciona "servicios". Cualquier tipo de comunicación en la capa de aplicación SDN o entre ésta y la capa de Gestión utiliza solamente este mensaje. La información transportada por los campos servicio y argumentos depende del conjunto de funciones proporcionado por cada RENESE y, por lo tanto, el protocolo es agnóstico de la semántica del servicio ofertado. La Figura 3.4 muestra un ejemplo del mensaje definido para el servicio de Seguimiento de Dispositivos (Device Tracking) utilizado en el caso de uso presentado en el capítulo de Validación de las Propuestas. 
Un mensaje RENESE se utiliza para cuatro propósitos diferentes: (i) descubrimiento de RENESE, (ii) suscribirse a un RENESE, (iii) solicitar una función específica del RENESE y (iv) enviar notificaciones a las aplicaciones. Antes de utilizar un servicio determinado, se debe llevar a cabo un proceso de suscripción entre la aplicación y el RENESE o entre dos RENESEs. Después de este proceso, la aplicación o RENESE pueden usar el servicio y recibir notificaciones desde el mismo. La Figura 3.4 muestra un ejemplo de modelo de interacción en el que la aplicación Estado de la Red (Network Status) se suscribe al servicio de Seguimiento de Dispositivos por medio del método síncrono de solicitud/respuesta y recibe notificaciones por medio del método asíncrono.

Considerando que más de una aplicación puede usar un servicio específico de esta capa y que todas las aplicaciones pueden realizar una acción sobre el mismo dispositivo de usuario, se requiere un método adecuado para evitar eventuales conflictos. Cuando el proceso de suscripción tiene lugar entre la aplicación y el servicio, la aplicación indica una prioridad en función del tipo de funcionalidad que se proporcionará. Posteriormente, el servicio utiliza esta prioridad para tomar decisiones sobre cómo tratar un determinado flujo de tráfico en el RGW basado en SDN.

Dentro de esta capa, el Gestor de RENESE (RENESE Manager) está a cargo de coordinar el intercambio de información de gestión que sigue un modelo "many-to-many" entre aplicaciones y servicios. Para coordinar este intercambio, el Gestor asigna un Identificador (Id) a cada RENESE y proporciona un servicio de descubrimiento que permite a las aplicaciones identificar el servicio requerido para suscribirse al mismo. Por otra parte, el Gestor también debe realizar un seguimiento sobre el estado de cada RENESE con el objetivo de comunicar eventuales fallos a las aplicaciones suscritas y ejecutar procesos de recuperación. En la sección 3.4.4 se proporciona información detallada sobre el funcionamiento de los servicios.

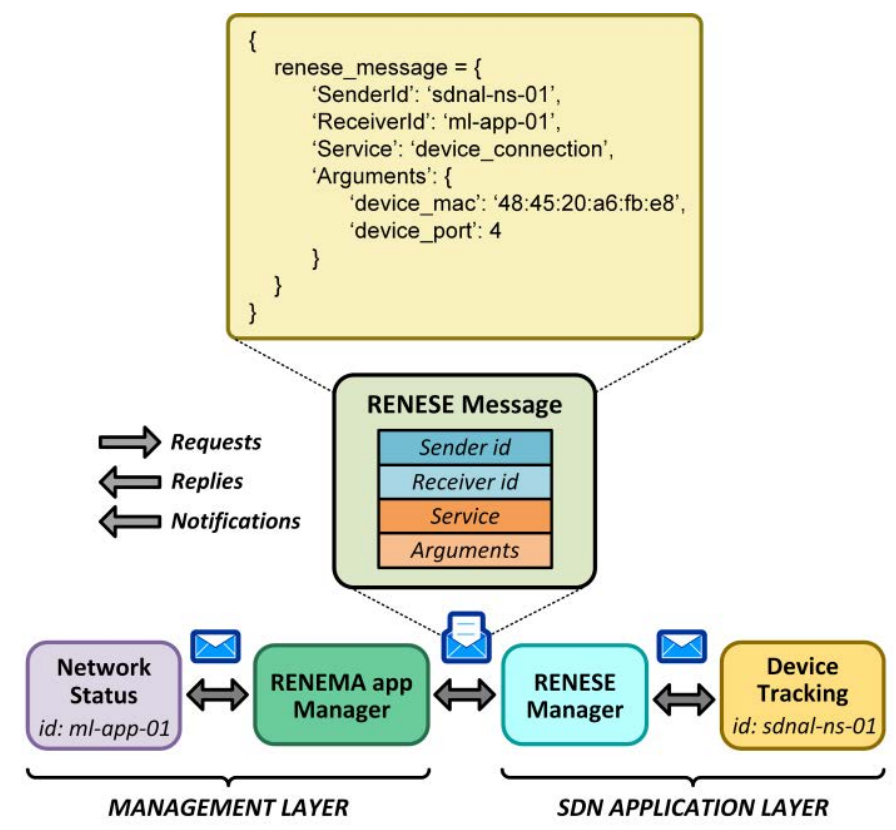

Figura 3.4 Modelo de interacción entre aplicaciones RENEMA y RENESEs. Ambos gestores ayudan en el intercambio de información de gestión utilizando para ello el protocolo RENESE. En la parte superior de la figura, se proporciona un ejemplo de un mensaje que utiliza un formato JSON ${ }^{1}$. El servicio de Seguimiento de Dispositivos notifica una "conexión de dispositivo" a las aplicaciones suscritas.

Siguiendo con la definición de funcionalidades que se proporcionan en esta capa, se presentan dos bloques funcionales adicionales: las Estadísticas de Red (NESA) que suministra información de estado y estadísticas sobre los recursos de red y, el Control de Recursos de Red (NERON) que se encarga de asignar los recursos de la red en función de la información proporcionada por NESA y los requisitos de

\footnotetext{
${ }^{1}$ JSON, acrónimo de JavaScript Object Notation, es un formato de texto ligero para el intercambio de datos.
} 
los usuarios residenciales (por ejemplo solicitar que el tráfico de videoconferencia tenga un porcentaje mayor de ancho de banda con respecto a los demás tipos de tráfico).

- NESA. Es posible que las aplicaciones necesiten determinadas métricas sobre el uso de los recursos de red como el caudal de tráfico, retardo, tasa de error o tipos de tráfico para proporcionar una funcionalidad específica. Esta información se origina desde diversos dispositivos de red o enlaces involucrados con diferentes niveles de agregación. El marco propuesto por Chowdhury et al. [85] aborda este tema y proporciona un enfoque interesante que muestra las posibilidades de SDN y, en particular, Openflow para recopilar estadísticas de red. El diseño e implementación de un nuevo marco de monitoreo específico no forma parte de los objetivos del diseño de la arquitectura centrada en el usuario, por el contrario, la propuesta es integrar un marco de monitoreo de terceros, como el mencionado anteriormente, con el objetivo de proporcionar las funcionalidades de NESA. No obstante, el marco adoptado deberá incluir, al menos, las siguientes funciones: una función de Monitoreo (Monitor) para sondear y recopilar métricas del RGW basado en SDN y, una función de Proveedor (Provider) que se encargaría de generar y entregar estadísticas usando un modelo bajo demanda.

- NERON. El ancho de banda de la conexión a Internet es un recurso limitado en la red residencial y debe gestionarse de forma apropiada. La provisión de QoS es un tema que ha sido estudiado exhaustivamente debido a su complejidad y, en consecuencia, se han presentado varios enfoques. La recomendación ITU-T Y.2111 [86] introduce las Funciones de Control de Admisión y Recursos (RACF) las cuales se encargan de gestionar los recursos de transporte en tiempo real en función de políticas y activadas por aplicaciones. Adicionalmente, el advenimiento del paradigma SDN ha permitido que nuevos enfoques, como los que proponen Bueno et al. [87] y Bari et al. [88], permitan que la provisión de QoS sea más flexible y efectiva. Considerando estos enfoques, este bloque funcional debería incluir, al menos, las siguientes funciones:

a. Las funciones de Control de Admisión y Planificación (ACS) están a cargo de tomar decisiones sobre la admisión de solicitudes de asignación de recursos con base en el estado actual de los recursos de la red. Cuando una aplicación solicita la provisión de QoS para un servicio, el ACS verifica la viabilidad de asignar recursos a la solicitud de QoS entrante. La provisión de QoS se traduce en la asignación de una cola específica en el puerto físico del RGW basado en SDN. De este modo, el ACS informa a la entidad solicitante acerca de la cola asignada y también configura el puerto físico para reflejar la nueva asignación realizada. Aunque OpenFlow no realiza la configuración de colas, se pueden utilizar diferentes protocolos, como OF-CONFIG [89], siempre que sean compatibles con el RGW basado en SDN.

b. La función de Contabilidad (Accounting) también es necesaria con el objetivo de medir, procesar y comunicar información sobre el consumo de recursos de red (ancho de banda). Para recopilar dicha información, esta función usa la función Proveedor de NESA. De este modo, se mantiene una visión clara y precisa de los recursos de la red. Las decisiones del ACS se basan en la información proporcionada por esta función y, por lo tanto, debe implementarse un alto nivel de precisión.

\subsection{Diseño del Dominio de Gestión y Redes Virtualizado}

\subsubsection{Contexto y Definición}

En la revisión del estado del arte se concluyó que una solución de gestión para redes residenciales híbrida compuesta por SDN y NFV representa un enfoque acertado. Si bien es cierto que un RGW basado en SDN ofrece varias ventajas en cuanto al control preciso de flujos de tráfico en comparación con el RGW tradicional o un dispositivo L2 simple utilizado en la propuesta de virtualización del RGW (vRGW), algunas funciones de red como el encaminamiento y NAT a cargo de proporcionar acceso a Internet a la red residencial, aún deben estar presentes. En este contexto, el enfoque de vRGW permite complementar la arquitectura de gestión centrada en el usuario propuesta en este capítulo. El paradigma NFV juega un papel importante en la externalización de las funciones de red desde el RGW basado en 
SDN a la infraestructura en la Nube del ISP como funciones de red virtualizadas (VNF) para garantizar la compatibilidad con el modelo de red tradicional.

Adicionalmente, con base en el principio $f$ previamente definido, un enfoque de gestión basado en la Nube, haría uso de NFV para desplegar VNFs especializadas y SFCs personalizadas por cada flujo de tráfico definido en el RGW basado en SDN para mejorar la provisión del servicio de conectividad de red con soporte de QoS. Por otra parte, la revisión del estado del arte permitió concluir que un enfoque de gestión basado en la Nube requiere un diseño específico del espacio virtual encargado de alojar a los componentes SDN y NFV, así como definir una ubicación adecuada para este espacio virtual que no afecte la capacidad de reacción del controlador SDN ni incremente la latencia de los servicios que se ejecutan en la red residencial debido a las tecnologías de virtualización utilizadas. Dillon y Winters [90] presentan un enfoque similar en el cual los paradigmas SDN y NFV se combinan para complementarse. Sin embargo, los autores se centran en mostrar el potencial práctico del enfoque, sin abordar el diseño detallado del plano de control SDN y del entorno virtual en la infraestructura del ISP que alojará los componentes SDN y NFV.

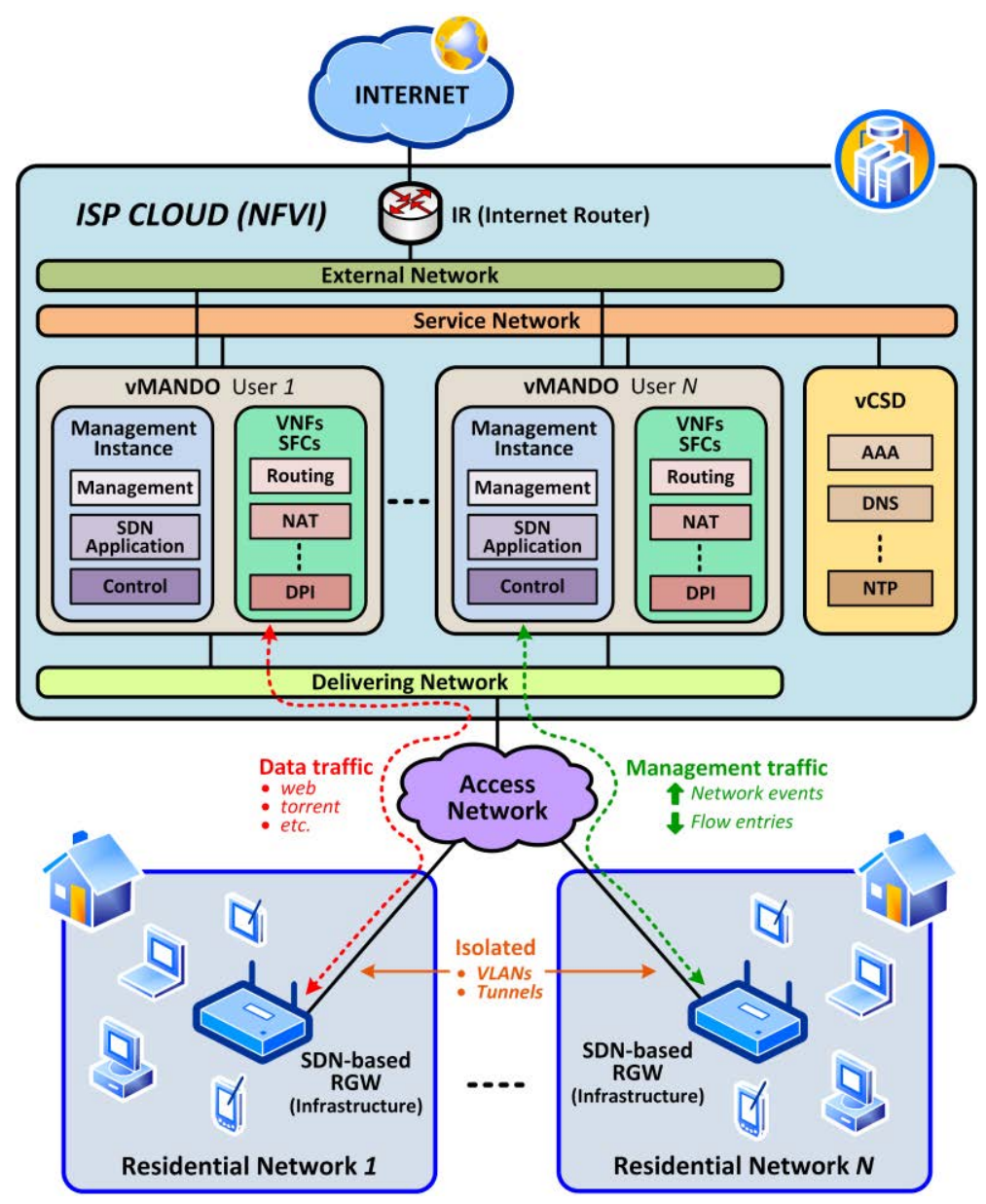

Figura 3.5 Estructura de vMANDO y su disposición en las instalaciones del ISP. Se crea un vMANDO por red residencial para alojar a la Arquitectura de Gestión SDN centrada en el usuario y diferentes VNFs.

Con esto en mente, se define el Dominio de Gestión y Redes virtualizado (vMANDO) el cual representa el espacio virtual asignado a la red residencial encargado de alojar a los componentes SDN y NFV a la vez que proporciona un entorno de ejecución para los mismos. Como se muestra en la Figura 3.5, el componente SDN está representado por la Instancia de Gestión (Management Instance) que incluye las capas de software de la arquitectura designadas como Gestión, Aplicación SDN y Control. En la red residencial, la capa Infraestructura está representada por un RGW basado en SDN y los diferentes clientes de aplicaciones RENEMA, que componen la capa Interacción, están instalados en diferentes dispositivos electrónicos como smartphones, tablets o PCs. Por otra parte, el componente NFV está 
representado por diferentes VNFs como encaminamiento, NAT o DPI (Deep Packet Inspection), así como las posibles SFCs que pueden configurarse con las mismas. Es importante mencionar que desde el punto de vista del paradigma NFV, la Instancia de Gestión representa otra VNF más, es decir, una VNF con la funcionalidad de gestionar el RGW basado en SDN.

Adicionalmente, también es importante mencionar que al implementar la función de encaminamiento en vMANDO, el dominio lógico de la red residencial se extiende hasta las instalaciones del ISP. Como lo indican Cruz et al. [55], este es el resultado de implementar el enfoque de vRGW. Si bien la red residencial está compuesta principalmente por el RGW como se indica en la arquitectura presentada en la sección 2.1.2, al desplazar la función de Gateway más allá del dominio físico, ciertamente se extiende el dominio lógico. De esta manera, resulta conveniente destacar que una red residencial basada en SDN y NFV está compuesta por un dominio físico y lógico y, por lo tanto, las funcionalidades de conectividad que ésta proporciona se distribuyen en ambos dominios.

La arquitectura de gestión centrada en el usuario y el concepto de vMANDO ciertamente proporcionan un nuevo paradigma de gestión, despliegue, mantenimiento y provisión de servicios para la red residencial caracterizado por proporcionar las siguientes ventajas:

- Al utilizar SDN y NFV, se proporciona un entorno ágil y flexible, caracterizado por el alto grado de automatización que se puede alcanzar en los procesos operativos asociados a la red residencial. De esta manera, el ISP es capaz de mejorar su nivel de competitividad a través de la innovación.

- La arquitectura de gestión se puede implementar y actualizar fácilmente ya que no está integrada en el RGW basado en SDN. Además, el uso de una interfaz Southbound estandarizada como OpenFlow permite que el RGW basado en SDN sea independiente del fabricante.

- Los recursos computacionales (procesamiento, almacenamiento o memoria) requeridos por los back-ends de las aplicaciones RENEMA no están limitados por el hardware del RGW basado en SDN, gracias al uso de tecnologías en la Nube.

- La propuesta de vMANDO es totalmente compatible con diferentes plataformas en la Nube y plataformas NFV. Por ejemplo, en el capítulo 5, se utilizó OpenStack para emular la Nube del ISP y desplegar diferentes vMANDOs con base en los requisitos de un caso de uso práctico. Adicionalmente, se realizó el ejercicio de implementar vMANDO como servicio de red (NS) y se obtuvieron los descriptores correspondientes utilizando TOSCA.

El paradigma NFV permite agregar varias VNFs de distinta complejidad a cada vMANDO utilizando un modelo bajo demanda. De esta manera, el usuario sería capaz de personalizar el componente NFV de vMANDO para enriquecer las funcionalidades que la red residencial puede proporcionar. Este enfoque se desarrolla en detalle en el siguiente capítulo al considerar el uso de SFCs para implementar un tratamiento de tráfico diferenciado con soporte de QoS en la red residencial.

\subsubsection{Redes de Soporte para vMANDO}

Ciertamente, la definición de vMANDO permite complementar la arquitectura de gestión propuesta al proporcionar un entorno virtual personalizable a cada red residencial. No obstante, con el objetivo de cubrir todas las funcionalidades actuales y futuras de la red residencial, cada vMANDO debe estar conectado a tres redes diferentes como se detalla a continuación y se muestra en la Figura 3.5:

- Red de Entrega (Delivering Network). Considerando que se utiliza un control "en banda", el tráfico de la red residencial que viaja a través de la red de acceso se compone de dos tipos de tráfico: gestión y datos. Dada la importancia del tráfico de gestión, éste debe aislarse del tráfico de datos. Mecanismos como VLANs o túneles se pueden usar para cumplir con este requisito y dirigir el tráfico a los componentes SDN y NFV correspondientes alojados en un vMANDO específico. Por ejemplo, se pueden definir dos VLANs (tradicionales o basadas en VXLAN) por red residencial. El tráfico etiquetado procedente de diferentes redes residenciales se concentraría en la Red de Entrega para posteriormente ser entregado a los componentes correspondientes (SDN o NFV) de un vMANDO dado. 
- Red de Servicios (Service Network). La compatibilidad con el paradigma de redes tradicional debe estar garantizado. Para este propósito, los servicios comunes utilizados en redes residenciales como DHCP, DNS, AAA (Authentication, authorization, and Accounting) o NTP (Network Time Protocol) se implementan en el Dominio de Servicios Comunes virtualizado (vCSD). Considerando que estos servicios son comunes para todas las redes residenciales, la Red de Servicios se encarga de proporcionar conectividad entre diferentes vMANDOs y el vCSD.

- Red Externa (External Network). El RGW tradicional se encarga de proporcionar acceso a Internet mediante el encaminamiento que realiza entre la red residencial y la red del ISP. Dado que esta función de red se ha re-ubicado en la Nube del ISP como una VNF, el vMANDO que aloja dicha VNF debe contar con conectividad a Internet. Además, la Red Externa permite conectar clientes de aplicaciones RENEMA que se ejecutan en dispositivos de usuario con sus respectivos back-ends en la Nube del ISP para gestionar la red residencial.

Debido a problemas de seguridad y complejidad, vMANDO proporciona un entorno virtual aislado. Con respecto a la seguridad, el tráfico de la red residencial (gestión + datos) debe aislarse a través de la provisión de componentes dedicados SDN y NFV. Así, en caso de que los componentes SDN o NFV se vean comprometidos, el fallo ocasionado puede ser delimitado, evitando su propagación y el colapso de todo el sistema. En cuanto a la complejidad, el diseño de una arquitectura de gestión centralizada a cargo de la gestión de múltiples redes residenciales resulta más complejo. Proporcionar a la red residencial de un entorno de gestión independiente resulta tan fácil como crear instancias de una nueva entidad de gestión dentro de un nuevo vMANDO, lo cual garantiza la escalabilidad. El componente NFV también podría estar centralizado para proporcionar encaminamiento, NAT y otras VNFs para todas las redes residenciales. Sin embargo, este enfoque puede crear un "único punto de falla". Por lo tanto, la provisión de vMANDOs independientes por red residencial representa una solución adecuada.

\subsection{Detalles Operacionales}

En las secciones previas, se presentó la propuesta de una arquitectura de gestión que permite implementar un enfoque centrado en el usuario y facilita su implementación para mejorar la usabilidad de las redes residenciales, hacer frente a la heterogeneidad existente y mejorar el servicio de conectividad de red provisto. En el proceso de definición de la arquitectura de gestión se ha proporcionado una descripción conceptual de las diferentes capas y sus correspondientes bloques funcionales. En esta sección se proporcionan detalles específicos sobre la operación o funcionamiento de la arquitectura que permitirán comprender de mejor manera las propuestas presentadas en esta tesis doctoral.

\subsubsection{Estados Operacionales}

Como se indicó anteriormente, diferentes aplicaciones RENEMA y RENESEs pueden ser agregados al entorno de gestión siguiendo un modelo bajo demanda. Dentro del entorno de gestión, una aplicación o RENESE pueden encontrarse en diferentes estados operacionales como se muestra en la Figura 3.6. Si el proceso de Inicio (Boot) resulta satisfactorio, se alcanza un estado Activo (Active) el cual representa el estado habitual en el que deberían encontrarse las entidades. El estado Activo permite que tanto aplicaciones como RENESEs desempeñen sus funciones de forma normal. En el caso de las aplicaciones, éstas permiten gestionar la red mediante el uso de las funciones que incorporan. En el caso de los RENESEs, éstos proporcionan servicios de configuración y monitoreo a las aplicaciones u otros RENESEs.

Del estado Activo se puede pasar a tres diferentes estados. Un fallo en la ejecución individual provoca una transición al estado de Error (Error). Un fallo en la aplicación impide realizar las tareas de gestión sin afectar al servicio proporcionado en la capa inferior. Por el contrario, un fallo del servicio o RENESE limitaría las funcionalidades de la aplicación, sin provocarle una transición al estado de Error, pero si al estado Detenido (Stopped). Un servicio también podría cambiar al estado Detenido si la capa de control deja de proporcionar determinadas funciones básicas. De forma general, se realiza una transición al 
estado Detenido cuando la entidad de la capa inferior deja de proporcionar las funcionalidades que debería.

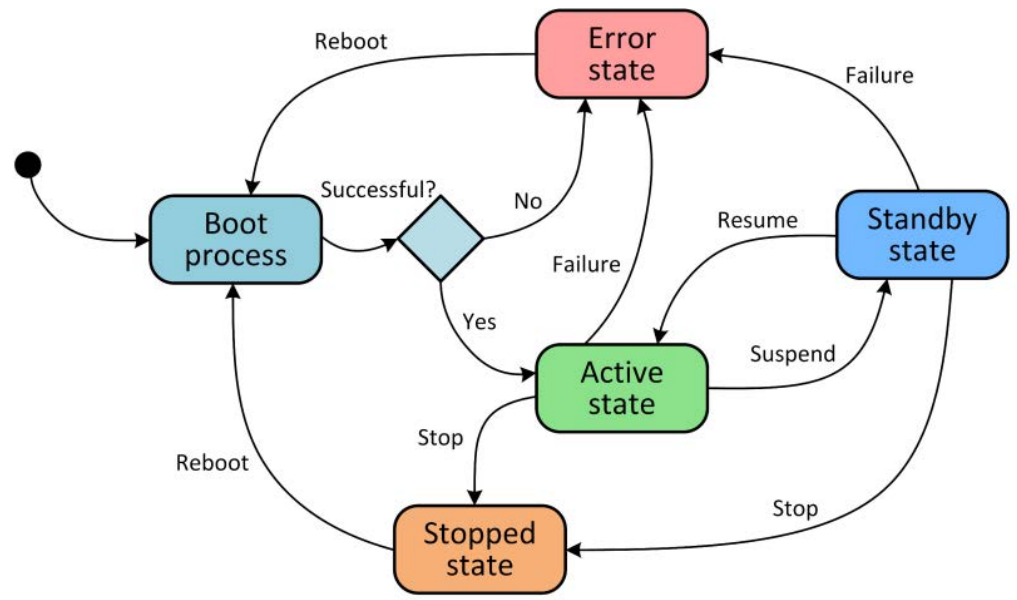

Figura 3.6 Estados operacionales de aplicaciones RENEMA y RENESEs.

Es responsabilidad de los Gestores, tanto a nivel de la capa de gestión como de la capa de aplicación SDN, informar sobre posibles fallos o detenciones detectados. Dependiendo de la implementación, en un caso, la aplicación o servicio podría ejecutar un proceso de Re-inicio (Reboot) por sí misma para salir del estado Detenido o de Error. En otro caso, el Gestor correspondiente podría ser el encargado de ejecutar tal proceso de Re-inicio.

Finalmente, también se considera la posibilidad de que tanto aplicaciones como RENESEs puedan cambiar al estado de Reposo (Standby) cuando no se han detectado actividades de gestión durante un largo período de tiempo. Este estado resulta útil para aplicaciones o servicios que demandan un alto consumo de recursos computacionales como es el caso del DPI (Deep Packet Inspection). Por otra parte, también permite que el ISP optimice el consumo de energía de las instancias virtualizadas considerando que su infraestructura alojará a varios cientos de ellas.

\subsubsection{Proceso de Inicio}

El proceso de Inicio permite que las aplicaciones y servicios alcancen el estado Activo. De forma general, en este proceso se distinguen tres etapas fundamentales. En primer lugar, se solicita la asignación de un Identificador (Id) al gestor de la capa correspondiente, si no existe y, se realiza un registro de la entidad solicitante. En segundo lugar, se realiza un proceso de descubrimiento de servicios requeridos, con base en el conjunto de funciones a proporcionar y, la posterior suscripción a los mismos. En tercer lugar, se establece una configuración de Inicio para la entidad. El proceso de Inicio determina el funcionamiento de la arquitectura siendo éste similar para aplicaciones y servicios. Además, este proceso permite definir el rol de consumidor o proveedor que tendrá un determinado servicio considerando que éstos pueden interactuar entre sí.

La Figura 3.7 muestra el proceso de Inicio para las aplicaciones RENEMA. En este proceso de distingue el soporte del correspondiente Gestor en la asignación de un Identificador para la aplicación. Como ejemplo, en el proceso de validación de la propuesta se ha utilizado un esquema para el Id compuesto por el nombre de la capa y el número de la aplicación. Una vez que el Id ha sido asignado, el Gestor registra la aplicación para proporcionarle los servicios de envío y entrega de mensajes. Para la etapa de descubrimiento, cada aplicación envía un mensaje con el objetivo de obtener el Id de un servicio en particular. En la Figura 3.7 se muestra un ejemplo de un mensaje de descubrimiento (service_discovery_request) en el cual se solicita el "Id" del servicio de Conmutación (Switching). El Gestor de aplicaciones RENEMA recibe el mensaje de descubrimiento creado por la aplicación para enviarlo a la capa inferior donde será recibido por el gestor de RENESEs quien a su vez retornará el Id solicitado. Posterior al descubrimiento, si la aplicación se ha iniciado por primera vez, puede que se 
solicite especificar determinados parámetros de funcionamiento si se los requiere, los cuales pueden ser modificados posteriormente y serán cargados cada vez que la aplicación inicie. Luego de realizar todo el proceso, la aplicación alcanza el estado Activo.

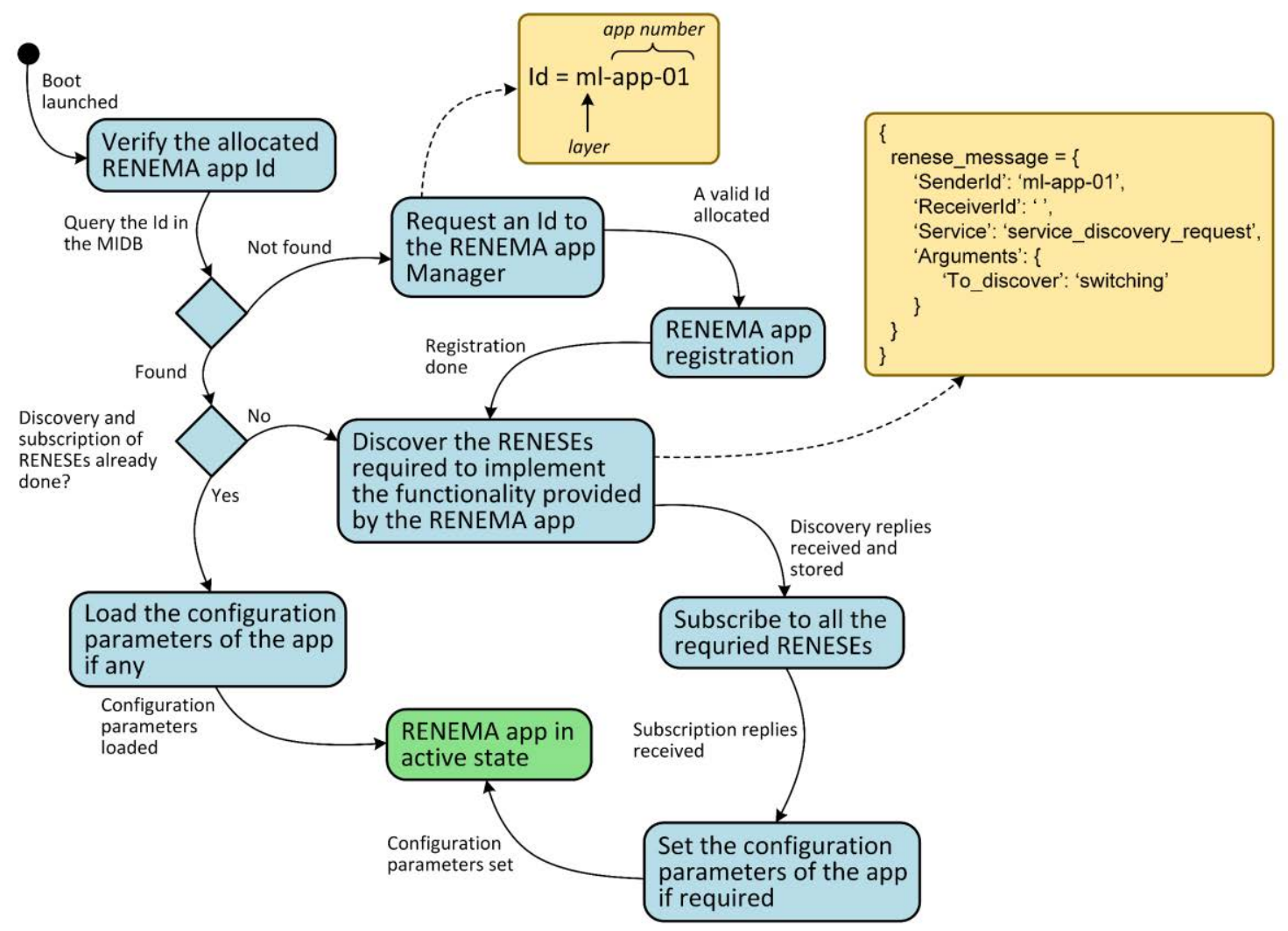

Figura 3.7 Diagrama de estados del proceso de inicio de una aplicación RENEMA.

La Figura 3.8 muestra el proceso de Inicio para un RENESE. Considerando que el proceso es similar al de una aplicación, se proporcionará una explicación de las rutinas adicionales que se necesitan. En el proceso de registro, por una parte, el Gestor registra el Id de la entidad para proporcionarle los servicios de envio, entrega y re-envío de mensajes. El proceso de re-envió se lo utiliza para intercambiar mensajes dentro de la misma capa de Aplicación SDN. Por otra parte, el Gestor registra el servicio proporcionado y el $I d$ de cada RENESE con el objetivo de formar una tabla de correspondencia la cual se utiliza en el proceso de descubrimiento de servicios. Por ejemplo, en la Figura 3.8 se observa que el servicio de Conmutación es proporcionado por la entidad "sdnal-ns-02".

Como se mencionó en la sección 3.2.5, la arquitectura también permite la composición de servicios dentro de la capa de aplicación SDN, de tal manera que un RENESE es capaz de suscribirse a otro para utilizar sus funciones. De esta manera, se realiza un proceso de descubrimiento si es necesario. Por ejemplo, como se verá en el capítulo 5, NERON (sdnal-ns-04) necesita obtener las estadísticas proporcionadas por NESA (sdnal-ns-01), por lo tanto, emite una solicitud de descubrimiento para obtener su Id y, posteriormente, suscribirse al servicio. En la Figura 3.8 se muestra la respuesta de descubrimiento que re-envía el Gestor a NERON. Una vez que ha finalizado el proceso de suscripción, a continuación, se obtienen los parámetros de configuración de la red residencial como la dirección MAC de la función de gateway, VLANs por defecto asignadas, puerto WAN, entre otros; los cuales son requeridos para el funcionamiento de determinados servicios. Esta configuración es realizada por el ISP y proporcionada al momento de desplegar e instalar la red residencial. Finalmente, el RENESE alcanza el estado Activo y es capaz de procesar solicitudes de suscripción o de función específica y enviar notificaciones en respuesta a eventos de red detectados.

Con respecto a los suscriptores del servicio, cada RENESE dispone de un registro local donde almacena el Id de la entidad a la cual está proporcionando un servicio. Cuando se recibe una solicitud de 
suscripción, el RENESE almacena el Id de la entidad solicitante. De esta manera, se aceptan solicitudes de servicio o envían notificaciones solamente a entidades suscritas. Cuando un servicio se inicia nuevamente, identifica sus suscriptores como paso previo antes de alcanzar el estado Activo.

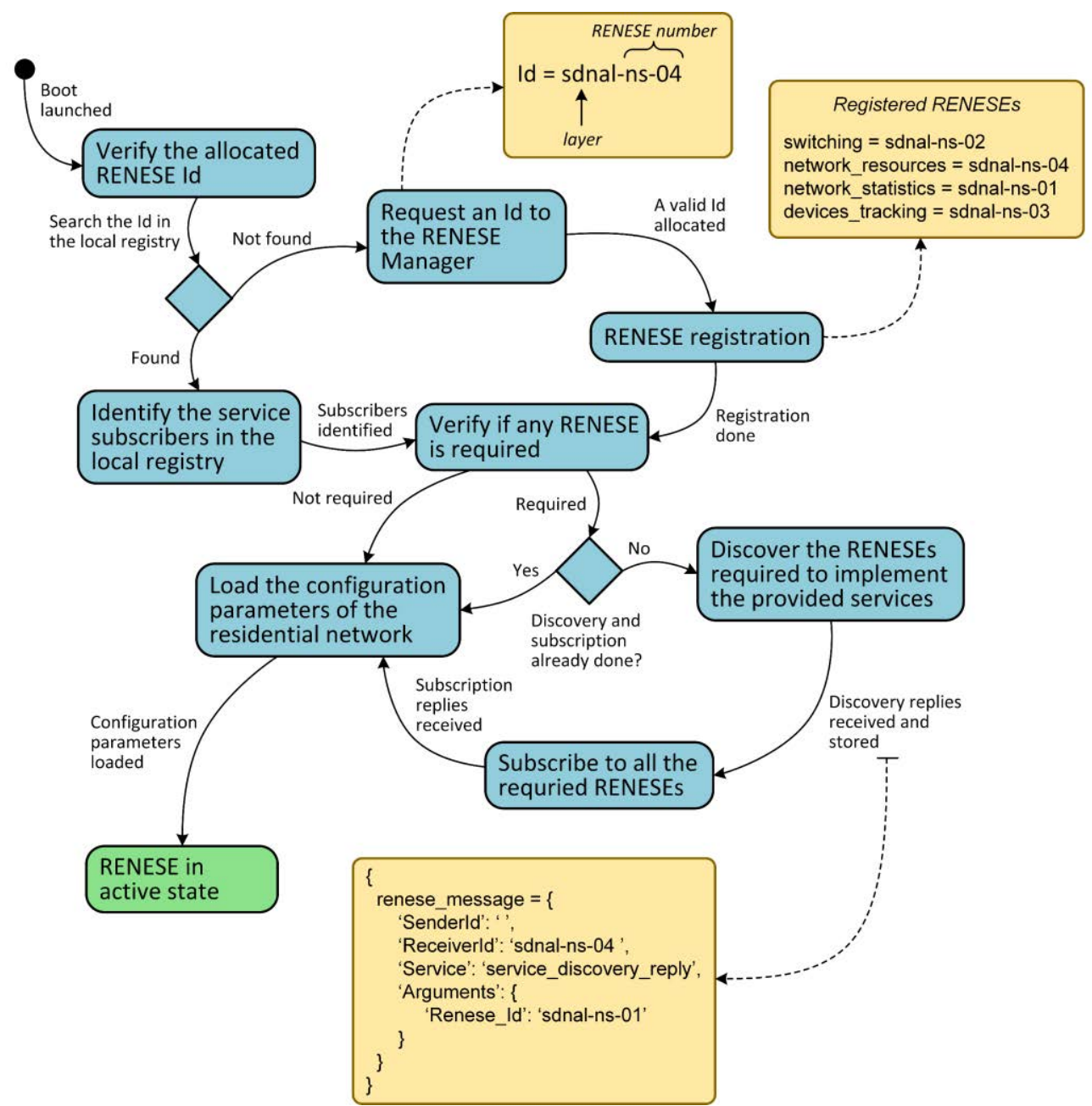

Figura 3.8 Diagrama de estados del proceso de Inicio de un Servicio de Red Residencial (RENESE).

\subsubsection{Funcionamiento de aplicaciones RENEMA}

En el estado Activo, de forma general, una aplicación debe procesar solicitudes realizadas a su API REST desde su respectivo cliente y mensajes enviados desde la capa de Aplicación SDN por un determinado RENESE. El conjunto de funciones que incorpora una aplicación determina el procesamiento que recibirán solicitudes y mensajes. En la Figura 3.9 se muestran los diferentes estados en los cuales se encuentra una aplicación para procesar solicitudes realizadas al API REST. La selección de una determinada función de la aplicación realizada en el cliente (capa de Interacción) origina una solicitud al API REST (capa de Gestión).

Las operaciones a realizar en respuesta a la llegada de una solicitud dependen del tipo de función seleccionada en el cliente. Las funciones de Tipo A pueden requerir que se realicen operaciones con la información que se recupera de la MIDB. Las operaciones a realizar son específicas de cada aplicación $\mathrm{y}$, por lo general, pueden requerir realizar cálculos adicionales con la información recuperada. De los cálculos realizados se obtienen resultados los cuales deben ser retornados con un formato apropiado al cliente de la aplicación.

Por otra parte, existen funciones de Tipo B que, aparte de poder requerir que se realicen operaciones con la información recuperada desde la MIDB, requieren que se realicen operaciones con base en el uso de funciones específicas de un RENESE al cual se encuentra suscrita la aplicación. Como se muestra en 
la Figura 3.9, dentro de las operaciones de las funciones de Tipo B, se requiere crear y posteriormente enviar un mensaje RENESE. Adicionalmente, el envío de tal mensaje puede requerir o no la creación y posterior envío de una respuesta al cliente de la aplicación.

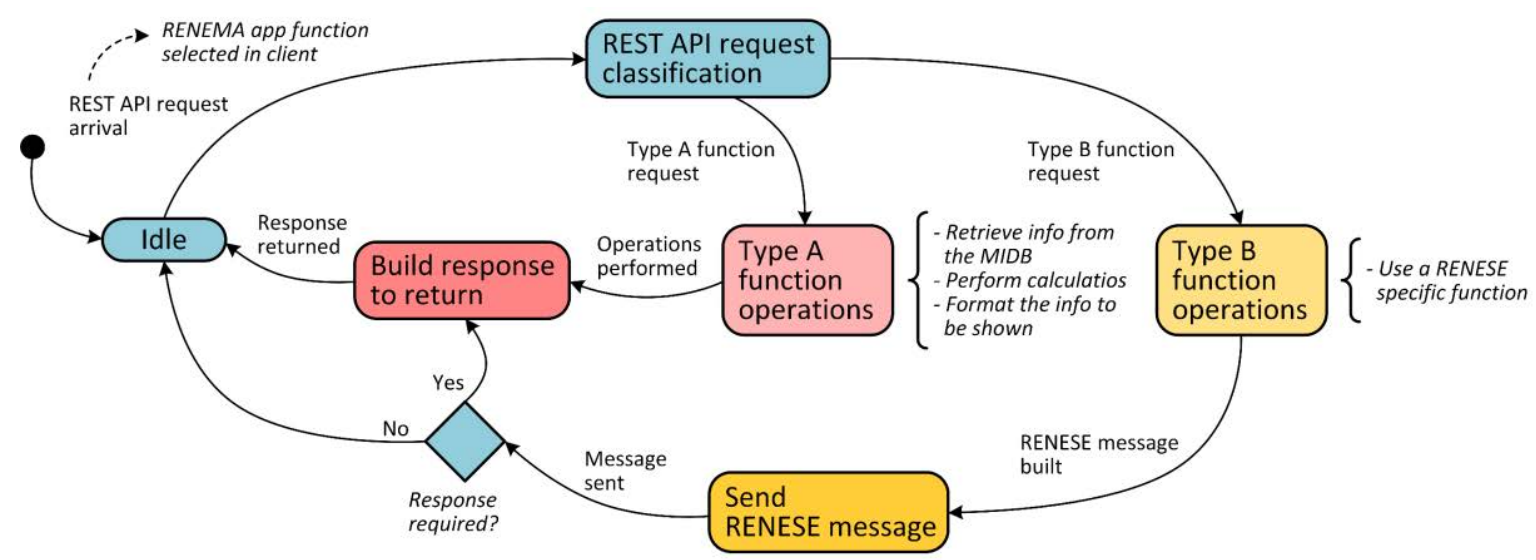

Figura 3.9 Diagrama de estados del funcionamiento de una aplicación de acuerdo con el procesamiento de solicitudes realizadas al API REST.

En lo que respecta al procesamiento de mensajes RENESE, la aplicación los clasifica en Respuestas y Notificaciones como se muestra en la Figura 3.10. En el procesamiento de Respuestas recibidas se distinguen tres tipos: respuesta al descubrimiento, respuesta a la suscripción y respuesta de una función especifica. En el procesamiento de Notificaciones, se distinguen dos tipos: A y B. Las notificaciones de Tipo A pueden requerir que se realicen operaciones con la información contenida en la notificación. Los resultados obtenidos de las operaciones realizadas serán almacenados en la MIDB. Una vez que las operaciones han sido realizadas, se puede requerir también enviar una notificación para informar al usuario a cerca del procesamiento realizado. Para este fin, se utiliza una notificación "push" considerando que es necesario enviarla desde la capa de gestión al cliente de la aplicación. Por ejemplo, la aplicación puede estar suscrita a un servicio que notifica la conexión o desconexión de dispositivos en la red residencial. Al recibir esta notificación, la aplicación puede no requerir el uso de una función específica del mismo u otro servicio. No obstante, la aplicación si requiere enviar una notificación "push" a su respectivo cliente.

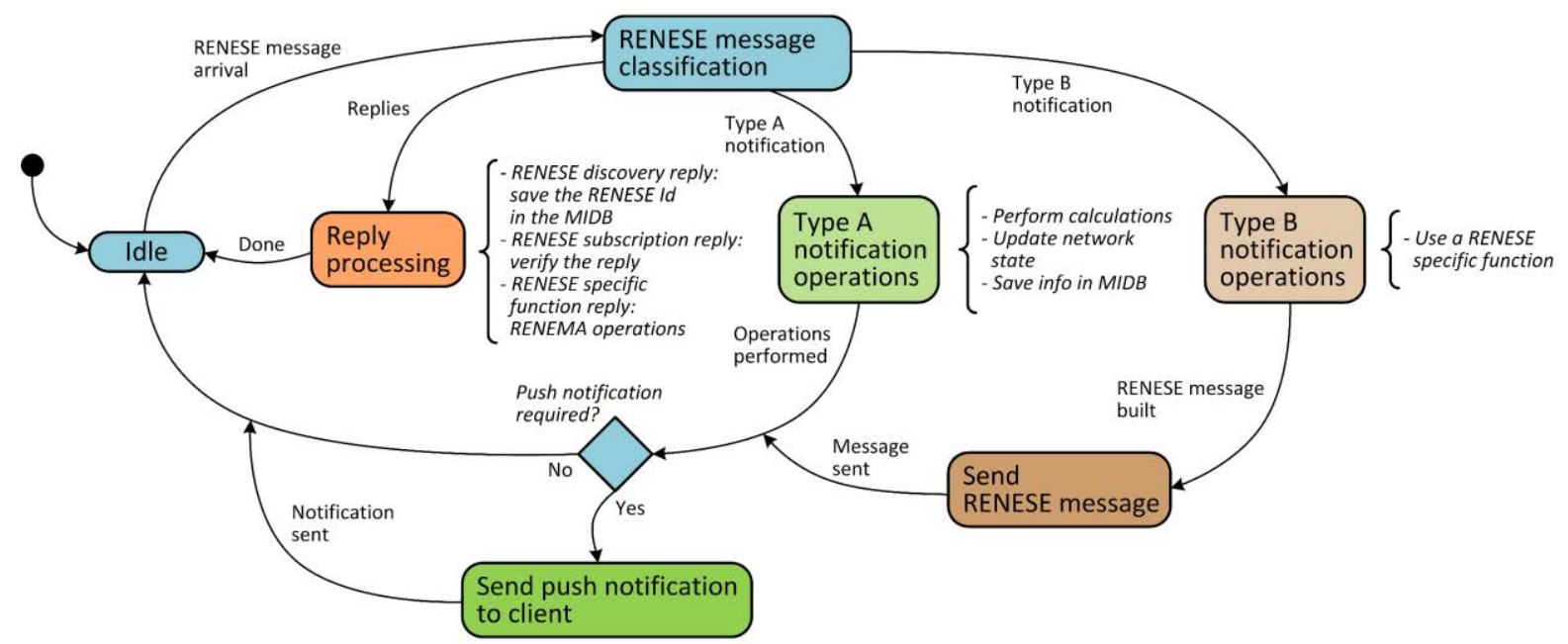

Figura 3.10 Diagrama de estados del funcionamiento de una aplicación de acuerdo con el procesamiento de mensajes RENESE. 
En el caso de las notificaciones de Tipo B, las operaciones a ser realizadas adicionalmente requieren utilizar una función específica de un RENESE al cual se encuentre suscrita la aplicación. Al igual que en el caso de las notificaciones de Tipo A, una vez que las operaciones realizadas con las notificaciones del Tipo B finalizan, también se puede requerir enviar una notificación "push" al cliente de la aplicación. Por ejemplo, puede existir otra aplicación que también esté suscrita al servicio de conexión/desconexión de dispositivos mencionado anteriormente. Así, al recibir una notificación, la aplicación puede requerir utilizar una función específica de otro servicio la cual proporciona conectividad de red al dispositivo de usuario recientemente conectado.

\subsubsection{Funcionamiento de RENESEs}

Los Servicios de Red Residencial, al alcanzar el estado Activo, deben procesar mensajes enviados desde aplicaciones y otros servicios, así como eventos de red detectados y notificados desde la Capa de Control. De forma general, el funcionamiento de un RENESE está determinado por el procesamiento que recibirán mensajes y eventos de red con base en el conjunto de funciones proporcionadas por cada servicio o RENESE. En la Figura 3.11 se muestran los diferentes estados en los que un servicio puede encontrarse para procesar un evento de red. Se destacan las posibles operaciones a realizar con los eventos de red recibidos. Una vez que las operaciones del servicio finalizan, se puede requerir o no el envío de una notificación a la aplicación u otro servicio con el fin de informar acerca del procesamiento realizado. Por ejemplo, un servicio determinado puede modificar el plano de datos para permitir la conectividad a Internet de un dispositivo de usuario y, por lo tanto, es necesario notificar a sus suscriptores sobre la operación realizada.

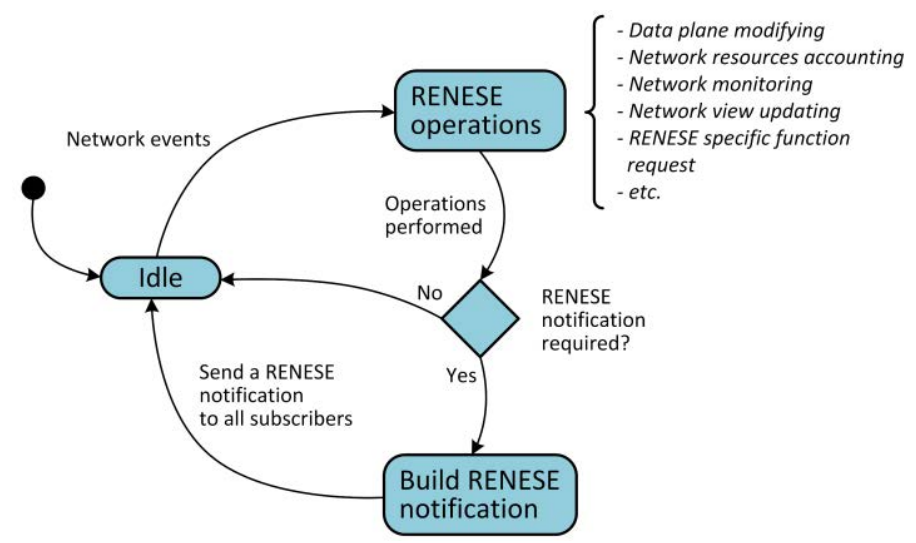

Figura 3.11 Diagrama de estados del funcionamiento de un RENESE de acuerdo con el procesamiento de eventos de red.

Con respecto al procesamiento de mensajes, cada RENESE realiza una distinción entre solicitud de suscripción y solicitud de función específica del servicio como se muestra en la Figura 3.12. Como se observó en la sección 3.4.2, el proceso de suscripción requiere agregar el Id de la entidad solicitante al registro de suscriptores local del servicio y posteriormente enviar una respuesta para confirmar tal suscripción. Por otra parte, las solicitudes de función específica requieren que el servicio realice determinadas operaciones en respuesta a su llegada. Una vez que las operaciones han sido realizadas, se puede o no requerir la modificación del plano de datos lo cual se traduce en la definición de entradas de flujo que deben ser enviadas a la capa de control para que finalmente sean instaladas en el RGW basado en SDN. Por ejemplo, pueden recibirse estadísticas de red a través de notificaciones y el RENESE suscrito a ellas puede no requerir realizar una modificación del plano de datos. Adicionalmente, se puede requerir que el servicio envíe una respuesta a la solicitud de función específica recibida. Por ejemplo, la función ACS de NERON puede recibir una solicitud de asignación de recursos y enviar una respuesta que especifica los recursos asignados sin necesidad de modificar el plano de datos.

Es importante mencionar que existen servicios que no precisan recibir eventos de red o mensajes para realizar una determinada operación. Por ejemplo, en la implementación práctica de la arquitectura 
realizada para validar las propuestas presentadas, NESA incorpora una función para recopilar estadísticas de red periódicamente que inicia conjuntamente con el servicio. Otras funciones que incorpora NESA, por el contrario, operan con base en los mensajes recibidos.

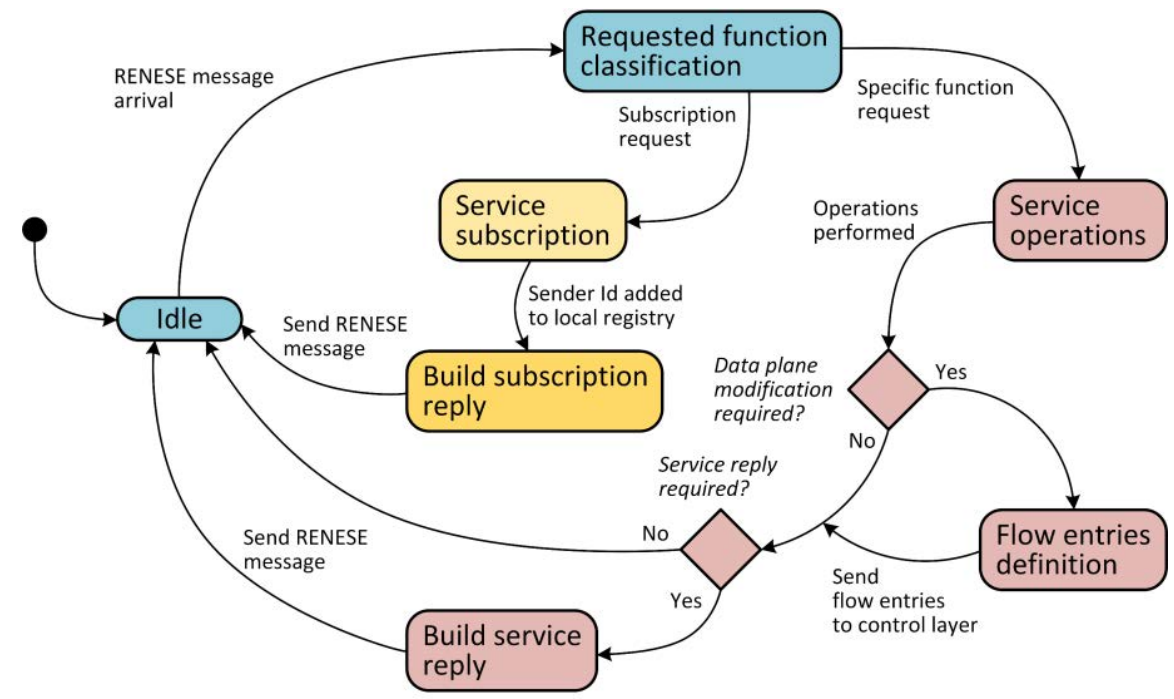

Figura 3.12 Diagrama de estados del funcionamiento de un RENESE de acuerdo con el procesamiento de mensajes.

\subsubsection{Participación de Gestores en el Intercambio de Información de Gestión}

Para comprender de mejor manera la participación de los Gestores en la coordinación del intercambio de información de gestión entre las capas de Gestión y Aplicación SDN o en la capa de Aplicación SDN solamente, la Figura 3.13 muestra tres diferentes casos de uso.

- En un primer caso, NERON inicia por primera vez y solicita al Gestor correspondiente la asignación de un Id (Id allocation request). Con el Id asignado, NERON se registra (Registration) en el Gestor y posteriormente requiere suscribirse a NESA. Suponiendo que NESA ya se ha registrado en el Gestor correspondiente, NERON envía una solicitud de descubrimiento (RENESE discovery request) para obtener el Id necesario. El Gestor recibe la solicitud, verifica su registro y envía una respuesta con el Id solicitado a NERON (RENESE discovery reply). Con el Id del servicio solicitado, se inicia el proceso de suscripción (Subscription request). NERON utiliza el servicio de re-envío proporcionado por el Gestor para enviar el mensaje a NESA (Forward message). El Gestor recibe el mensaje, verifica el destinatario y lo entrega. Una vez que se registra el Id del solicitante (Add subscriber), NESA utiliza el servicio de re-envío para enviar una respuesta a NERON (Subscription reply).

- En un segundo caso, una aplicación inicia por primera vez y solicita al Gestor la asignación de un Id. Una vez que la aplicación se ha registrado, se inicia el proceso de descubrimiento del RENESE A. Considerando que este servicio se encuentra en estado Activo, la aplicación utiliza el servicio de envío de mensajes proporcionado por el Gestor para enviar una solicitud de descubrimiento (Send message). La solicitud es recibida por el Gestor de RENESEs y envía una respuesta con el Id solicitado (RENESE discovery reply). En la capa de Gestión, la respuesta es recibida por el Gestor y posteriormente entregada a la aplicación (Deliver message). En una etapa final, la aplicación se suscribe al RENESE A, enviando una solicitud a través del Gestor. A su vez, el RENESE envía una respuesta a través de su correspondiente Gestor.

- En un tercer caso, suponiendo que la aplicación se encuentra en estado Activo, al recibir una solicitud desde el cliente (REST API request), se requiere utilizar una función específica del RENESE A para modificar el plano de datos. Para este fin, la aplicación crea un mensaje solicitando una función específica y la envía a la capa de Aplicación SDN utilizando el servicio 
de envío del Gestor (RENESE specific function request). En la capa de Aplicación SDN, el Gestor recibe el mensaje y se lo entrega al RENESE A para que lo procese y modifique el plano de datos.

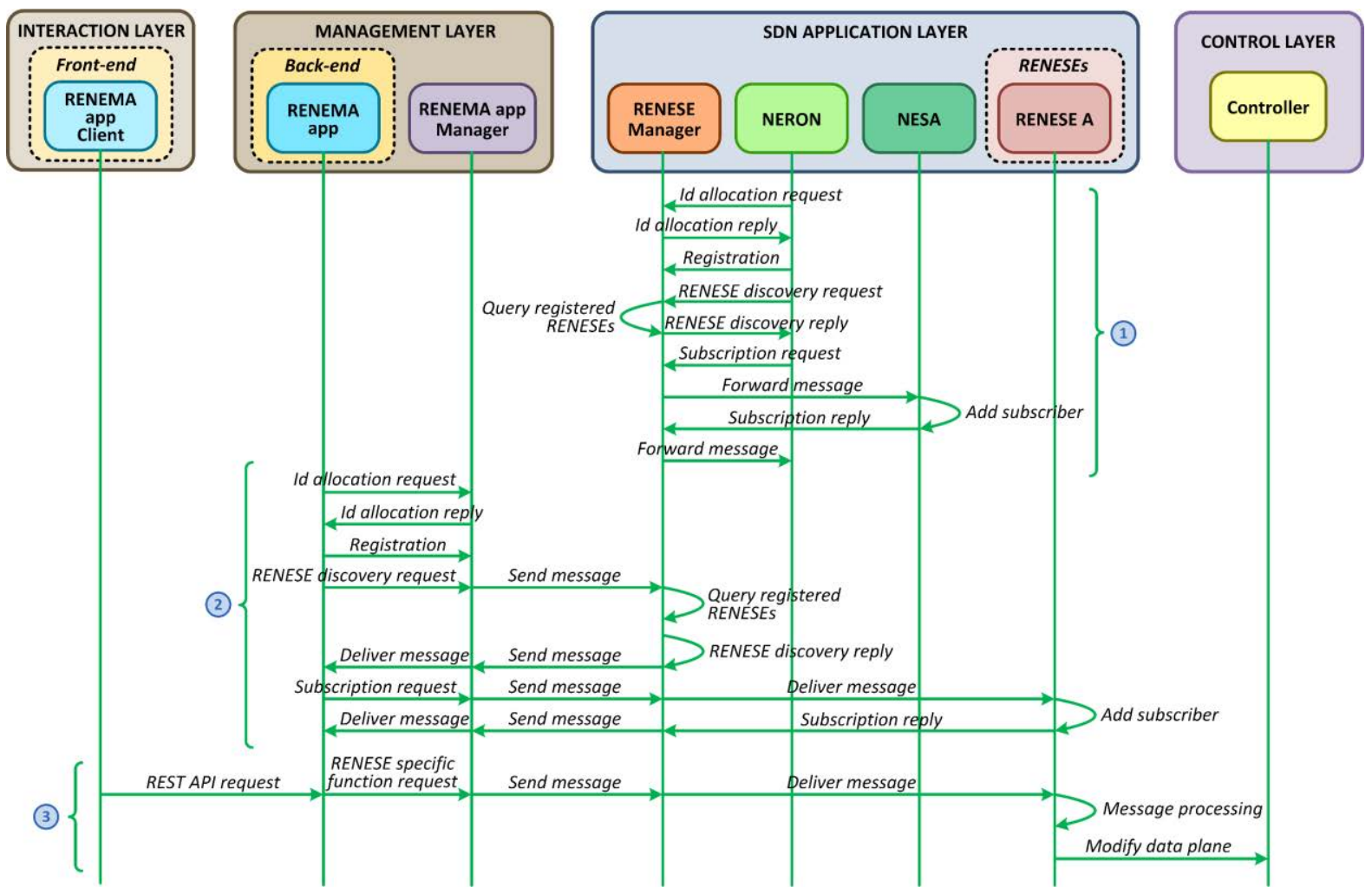

Figura 3.13 Participación de los Gestores de las capas de Gestión y Aplicación SDN en la coordinación del intercambio de información de gestión.

\subsection{Análisis de las Propuestas}

La propuesta de una arquitectura de gestión integral que combina los paradigmas SDN y NFV representa una mejora significativa en comparación con el modelo actual de las redes residenciales. Por una parte, se aborda el problema de la usabilidad de la red y la alta heterogeneidad del entorno al proporcionar diferentes aplicaciones adaptadas a las destrezas técnicas de un usuario promedio y encargadas de mejorar un determinado aspecto de la red residencial. Por otra parte, el concepto de vMANDO aborda el problema de diseño de un enfoque de gestión basado en la Nube conjuntamente con el paradigma NFV para aprovechar las ventajas con respecto a la escalabilidad, agilidad y flexibilidad que brindan estas tecnologías en la provisión de nuevas funcionalidades de red para enriquecer el servicio de conectividad que proporciona la red residencial. Adicionalmente, es importante destacar la posibilidad de agrupar todos los componentes SDN y NFV que soportan la red residencial en vMANDO lo cual permite automatizar los procesos de despliegue, gestión y mantenimiento con fines de optimización. Esta sección presenta un análisis de las propuestas considerando los siguientes temas de interés:

- Un nuevo paradigma para la red residencial. Como lo afirman Edwards et al. [3], no existe un modelo de red específico para las redes residenciales. El enfoque centrado en el usuario no solo aborda este problema, sino que también pretende proporcionar un nuevo paradigma de gestión y provisión de servicios a cargo de abordar los requisitos actuales y futuros en consonancia con las tecnologías SDN y NFV que sustentan el modelo de red moderno. En este contexto, la arquitectura de gestión propuesta representa un medio para conseguir la centralidad en el usuario.

- Una arquitectura abierta. Debido a la arquitectura cerrada de los RGWs tradicionales, la adición de nuevas funcionalidades requiere una actualización de firmware y el rendimiento de las funcionalidades avanzadas puede verse afectado por los recursos computacionales limitados del 
hardware. Un enfoque basado en la Nube y en el paradigma NFV permite proporcionar una arquitectura de gestión abierta para personalizar fácilmente el entorno de gestión con diferentes aplicaciones RENEMA y mejorar las funcionalidades de la red residencial mediante la adición de VNFs especializadas. En este contexto, aplicaciones y VNFs serían creadas por diferentes desarrolladores y funcionarían con una amplia variedad de RGWs basados en SDN y plataformas de NFV, evitando el efecto de bloqueo del fabricante y enriqueciendo el catálogo de servicios disponibles para la red residencial.

- Diferentes servicios para diferentes aplicaciones. Como se menciona en la arquitectura de referencia [22], la capa de aplicación SDN se encarga de especificar el comportamiento de los elementos de red mediante diferentes aplicaciones SDN. La arquitectura propuesta ha desarrollado aún más este principio mediante el concepto de Servicios de Red Residencial. De esta manera, al igual que un enfoque de kit de herramientas, varios servicios con funcionalidades específicas son utilizados a través del protocolo RENESE con el objetivo de crear una aplicación para gestionar la red. Por otra parte, además de los diversos servicios que se pueden proporcionar en la capa de aplicación SDN, NERON y NESA desempeñan un papel fundamental ya que sus funcionalidades permiten superar las limitaciones relativas a la provisión de QoS y monitoreo de red que las redes residenciales presentan actualmente.

- Compatibilidad con diferentes plataformas de NFV. La propuesta de vMANDO describe cómo la Nube del ISP podría contener a los componentes SDN y NFV utilizando dominios virtualizados como entornos de ejecución. La propuesta presentada ha utilizado el paradigma de NFV como complemento y, por lo tanto, está alineado con el marco arquitectónico de NFV propuesto por ETSI [39] y los procesos de Gestión y Orquestación (MANO) de NFV correspondientes [42]. En consecuencia, considerando que la Nube del ISP representa una NFVI, diferentes proyectos y soluciones propietarias enfocados en abordar los desafíos con respecto a NFV-MANO, como los que se analizan en la sección 2.3.6, se pueden integrar en la propuesta teniendo en cuenta los requisitos específicos del enfoque de vMANDO.

- Mejor utilización de la infraestructura del ISP. Tanto el dominio del usuario como el del ISP se ven afectados por la propuesta de vMANDO. Sin embargo, las modificaciones que se realizarán no incrementan el costo por residencia, ya que un dispositivo simple habilitado para OpenFlow reemplaza al tradicional RGW (un dispositivo de red más complejo y también más costoso). En cuanto a los ISPs, la adopción de Nubes privadas para optimizar sus entornos de IT (Tecnologías de la Información) ha experimentado un crecimiento significativo en los últimos años. Este hecho respalda la propuesta presentada considerando que las infraestructuras en la Nube actualmente desplegadas, las cuales se encargan de proporcionar funcionalidades de configuración remota del RGW, pueden ser utilizadas de mejor manera. Por ejemplo, el Portal Alejandra ${ }^{2}$ utilizado por Movistar en España a cargo de proporcionar tales servicios de configuración remota podría actualizarse para incorporar un sistema de gestión integral que incluya herramientas avanzadas orientadas al usuario para controlar la red residencial. Además, la infraestructura en la Nube se puede ampliar para cubrir los nuevos requisitos. La inversión estaría justificada por los nuevos y mejores servicios que se proporcionarán.

- Orquestación conjunta de SDN y NFV. Los componentes SDN y NFV de la arquitectura de gestión deben trabajar de forma colaborativa para cumplir con éxito los requisitos de conectividad y procesamiento de tráfico que imponen determinadas aplicaciones que se ejecutan en la red residencial. Por ejemplo, el usuario podría solicitar que el tráfico P2P (peer-to-peer), como el de BitTorrent $^{3}$, sea evaluado por una función de DPI o agregar un filtro de contenido al tráfico web que se accede desde los dispositivos de los niños en casa. En estos casos, una aplicación RENEMA debería encargarse de estos requerimientos y necesitaría realizar configuraciones tanto a nivel NFV como SDN. Por ejemplo, la aplicación permitiría agregar VNFs adicionales y crear SFCs específicas al mismo tiempo que indicaría al RGW basado en SDN como dirigir el tráfico a una SFC determinada. En este contexto, es necesario formalizar mecanismos adicionales para orquestar

\footnotetext{
${ }^{2}$ http://www.movistar.es/particulares/internet/adsl-fibra-optica/clientes/configuracion-routers-portal-alejandra/

${ }^{3}$ BitTorrent es un protocolo utilizado para el intercambio de archivos P2P en Internet
} 
conjuntamente los componentes SDN y NFV, así como nuevas interfaces para intercambiar información de gestión tal y como lo propone ONF [91] y Matias et al. [92]. Este requerimiento se aborda en el siguiente capítulo al proponer que desde la arquitectura de gestión centrada en el usuario se acceda a las interfaces MANO de la plataforma de NFV con el objetivo de orquestar el despliegue de VNFs y SFCs dentro de cada vMANDO.

- Un diseño SDN/NFV apropiado. Un enfoque basado en la Nube y en NFV, sin duda proporciona ventajas con respecto a la asignación dinámica de vMANDOs y sus correspondientes recursos computacionales y de redes. Sin embargo, se deben considerar los desafíos a superar cuando se aplican tecnologías de virtualización al entorno de los operadores de telecomunicaciones. Como lo indican Kim et al. [93], se deben analizar en detalle diferentes parámetros operacionales como el rendimiento o la confiabilidad para proporcionar patrones de diseño de arquitectura adecuados antes de implementar SDN y NFV. De forma similar, como lo mencionan Mijumbi et al. [36] y Han et al. [94], el concepto de NFV debe cumplir con las principales consideraciones de diseño como seguridad, disponibilidad, escalabilidad y estabilidad, para que se considere una solución aceptable para los operadores de telecomunicaciones. Si bien es cierto que NFV aprovecha algunas ventajas de la computación en la Nube como la escalabilidad o la rápida implementación de servicios, los entornos en la Nube deben evolucionar para obtener un comportamiento de "clase operador" que se ajuste a los requisitos que imponen las redes de la actualidad. El concepto EPA (Enhanced Platform Awareness) [95] representa un avance importante en la provisión de un entorno de Nube a la medida de los operadores de telecomunicaciones. La posibilidad de especificar requisitos de hardware para aplicaciones o funciones de red que se ejecutan en máquinas virtuales permite una adaptación precisa de los requisitos de carga de trabajo a las capacidades mejoradas de la plataforma, mejorando significativamente el rendimiento y aproximándolo a valores no virtualizados.

Por otra parte, los aspectos relacionados con las plataformas de virtualización, arquitectura de red o disponibilidad de recursos virtualizados deben evaluarse en detalle para no afectar el rendimiento cuando SDN y NFV se implementan conjuntamente en los centros de datos en la Nube según lo indica el Grupo de Investigación en SDN (SDNRG) [96]. Enfoques como estos intentan proporcionar al concepto de NFV la confiabilidad, estabilidad y robustez requeridas, así como lograr un equilibrio entre rendimiento y costo.

- Capacidad de la Nube del ISP y latencia del servicio. Considerando el despliegue masivo de vMANDOs, la Nube centralizada del ISP podría no ser lo suficientemente eficiente como para satisfacer todas las demandas de los clientes y garantizar un nivel adecuado de calidad de servicio. Por otra parte, también es importante considerar que las VNFs que se despliegan en cada vMANDO y las diferentes SFCs configuradas pueden incrementar significativamente la latencia del servicio, debido al tiempo de procesamiento adicional que introducen las capas de virtualización. Este incremento en la latencia no solamente afecta a los servicios que se consumen en casa del usuario sino también podría afectar a la capacidad de respuesta de las aplicaciones RENEMA que se ejecutan en vMANDO. De esta manera, se han propuesto diferentes enfoques para abordar estos temas. Por ejemplo, Qu et al. [97] proponen un modelo para la planificación de VNFs que considera tanto los retardos en el procesamiento de VNF que se ejecuta en una determinada máquina virtual como los retardos en los enlaces virtuales de la SFC que realiza la transmisión de tráfico con el objetivo de mejorar el rendimiento general de la planificación y cumplir con los requisitos de retardo estrictos de determinados servicios. Por otra parte, Krishnaswamy et al. [98] proponen una jerarquía de centros de datos distribuidos para ubicar de manera flexible VNFs en diferentes partes de la jerarquía en función de requisitos de rendimiento como latencia, costo o disponibilidad de recursos.

Similarmente, el concepto de Nube de Borde (Edge Cloud) mencionado por Chang et al. [99] o Computación en el Borde (Edge Computing) mencionado por Shi y Dustdar [100] representan una alternativa novedosa a los centros de datos centralizados ubicados en la red central del ISP. Una Nube de Borde proporciona tiempos de respuesta más cortos, reduce el ancho de banda del tráfico de la red troncal y el consumo de energía, así como proporciona una etapa inicial para el procesamiento del tráfico. CORD [61] representa un ejemplo de uso de una Nube de Borde e inclusive puede también ser considerado como un ejemplo de distribución jerárquica de centros de datos. Siguiendo este enfoque, los recursos en la Nube del ISP se pueden distribuir colocando 
conjuntamente una plataforma en la Nube con el nodo de acceso en diferentes oficinas centrales. Así, cada Nube de Borde estaría a cargo de alojar un determinado número de vMANDOs con base en la disponibilidad de recursos computacionales y los requisitos de los servicios sensibles a la latencia.

- Escalabilidad de SDN. Con el objetivo de garantizar un rendimiento óptimo de la arquitectura de gestión, es importante considerar los problemas de escalabilidad asociados a SDN y sus soluciones correspondientes. La literatura indica que existen tres tipos de problema. El primero está relacionado con el retardo de la instalación de una entrada de flujo en el dispositivo SDN. Un aumento excesivo de este retardo podría crear un cuello de botella que limita la capacidad de respuesta en tiempo real del plano de control. En el capítulo 5, se analiza en detalle este tipo de retardo, se realizan pruebas específicas para medir el impacto de la carga de tráfico y se proponen mecanismos para limitar su incremento y mantener un valor constante.

El segundo problema de escalabilidad está relacionado con la capacidad del plano de control para procesar una gran cantidad de flujos por segundo. Kreutz et al. [32] indican que las diferentes soluciones del plano de control abarcan arquitecturas distribuidas y multinúcleo para abordar este problema y aumentar la escalabilidad de SDN. Así, algunas soluciones son capaces de proporcionar una capacidad de procesamiento de 20 millones de flujos/s en determinadas condiciones de trabajo.

El tercer problema de escalabilidad está relacionado con la capacidad del plano de datos para almacenar una gran cantidad de entradas de flujo. Rygielski at al. [101] mencionan que este problema se aborda utilizando una TCAM (Ternary Content Addressable Memory) para implementar tablas de flujo en hardware que permitan ofrecer una velocidad de conmutación de línea cercana a la que proporciona un conmutador en hardware. Sin embargo, una TCAM tiene una capacidad y prestaciones muy limitadas debido a que esta memoria es costosa y tiene un alto consumo de energía. Para abordar esta limitación, se propone reducir el número de entradas de flujo en la TCAM. Así, Kreutz et al. [32] indican que algunos autores proponen técnicas de compresión mientras que Zhang et al. [102] proponen utilizar métodos para establecer de forma adaptativa un tiempo de espera adecuado para cada entrada de flujo.

Adicionalmente, pruebas de rendimiento especializadas como las que presentan Kuźniar et al. [103] pueden ayudar a los desarrolladores de aplicaciones de controlador y arquitectos de red a mejorar sus diseños sin requerir configuraciones experimentales costosas y exigentes.

- Estandarización. Diferentes grupos de trabajo han sido creados por las Organizaciones de Desarrollo de Estándares (SDOs) y foros de la industria con el fin de especificar los componentes funcionales de las arquitecturas SDN y NFV. Las propuestas presentadas en este capítulo no solo representan un esfuerzo por contribuir a la estandarización de las futuras redes residenciales, sino que también pueden integrarse como elementos funcionales en las propuestas de los diferentes grupos de trabajo. A continuación, se consideran dos posibles casos de aplicación:

En primer lugar, la arquitectura propuesta garantiza que tanto las aplicaciones RENEMA como los RENESEs puedan evolucionar de forma independiente. Con el objetivo de obtener un resultado similar, la propuesta de Arquitectura Cooperativa en Capas para SDN [104] del grupo de Investigación en SDN (SDNRG) separa las funciones relacionadas con el transporte de las funciones relacionadas con el servicio. Sin embargo, la propuesta de SNDRG no especifica cómo proporcionar servicios a aplicaciones externas. En este contexto, el protocolo RENESE podría considerarse una opción adecuada ya que describe cómo las aplicaciones SDN acceden a los servicios del controlador y cómo las aplicaciones SDN pueden proporcionar servicios a entidades externas o capas superiores. En segundo lugar, la propuesta del grupo de trabajo I2NSF (Interface to Network Security Functions) del IETF [105] también define un conjunto de interfaces de software y modelos de datos para controlar y monitorear aspectos de NSFs físicos y virtuales, permitiendo a los clientes especificar conjuntos de reglas. Sin embargo, la propuesta de I2NSF podría mejorarse considerando el enfoque basado en aplicaciones que propone la arquitectura centrada en el usuario. De esta forma, las funciones de seguridad de la red pueden implementarse como aplicaciones de código abierto para ser independientes de las funciones disponibles de fabricantes específicos. 


\subsection{Conclusiones}

Gestionar una red residencial hoy en día requiere un cierto nivel de experiencia técnica que resulta demasiado exigente para los usuarios residenciales, lo cual afecta negativamente a la usabilidad de la red residencial. En este capítulo, se ha propuesto un nuevo modelo de gestión para la red residencial considerando un enfoque centrado en el usuario con el objetivo de mejorar la usabilidad o capacidad de gestión de la red, abordar el tema de la alta heterogeneidad de este tipo de entornos y enriquecer el servicio de conectividad de red que se proporciona. El principal problema a abordar con este enfoque es determinar una estrategia adecuada que permita integrar a los usuarios en las tareas de gestión de sus propias redes. De esta manera, se ha definido la aplicación para la Gestión de la Red Residencial (aplicación RENEMA) la cual está diseñada pensando en los requisitos y las habilidades de los usuarios residenciales. Por otra parte, un enfoque de gestión basado en aplicaciones impone determinados requisitos al gateway residencial (RGW) como la capacidad de controlar de forma precisa el recorrido del tráfico o la facilidad para comunicar eventos de red con el fin de mejorar un determinado aspecto de la red residencial. En este contexto, surge la necesidad de definir una arquitectura que satisfaga los requerimientos de las aplicaciones a la vez que facilite su desarrollo y permita proporcionar un modelo específico para la red residencial en consonancia con las tecnologías SDN, NFV y la Nube.

La arquitectura de gestión centrada en el usuario propuesta ha requerido una mayor definición y especificación de la arquitectura de referencia SDN, así como la definición de un conjunto de principios para proporcionar un entorno de gestión integral, flexible y capaz de evolucionar en el tiempo que puede contribuir en el proceso de estandarización de un nuevo modelo de red para las redes residenciales. La arquitectura propuesta pone a disposición un conjunto de Servicios de Red Residencial (RENESEs) para ocultar los detalles de configuración y monitorización de red de bajo nivel con el objetivo de facilitar y agilizar el desarrollo de aplicaciones RENEMA, así como fomentar su desarrollo y reducir la dependencia de los fabricantes.

El concepto de Dominio de Gestión y Redes virtualizado (vMANDO) al integrar los principios de NFV permite que el usuario también sea capaz de incluir nuevas Funciones de Red Virtualizadas (VNFs) para configurar diferentes Cadenas de Función de Servicio (SFCs) destinadas a ampliar las funcionalidades que la red residencial puede ofrecer. Por otra parte, vMANDO resulta de especial interés para el ISP ya que al integrar los componentes SDN y NFV en una única entidad virtualizada, se dispone de un entorno ágil y flexible para orquestar servicios asociados a la red residencial.

Ciertamente, la arquitectura propuesta proporciona ventajas en comparación con el modelo actual de las redes residenciales. No obstante, con el objetivo de proporcionar una solución factible, eficiente y fiable es importante considerar que SDN y NFV, a pesar de sus características valiosas, son tecnologías relativamente nuevas que se encuentran en constante evolución y, por lo tanto, presentan determinadas limitaciones. Así, es importante mantener un conocimiento actual de las soluciones que se proponen desde la industria y la academia destinadas a superar estas limitaciones con el objetivo de sustentar las propuestas presentadas en este capítulo. 


\section{Capítulo 4}

\section{Tratamiento de Tráfico Diferenciado basado en SDN y NFV}

En la actualidad, lograr que los servicios o aplicaciones multimedia que se ejecutan en casa del usuario y que utilizan los servicios de conectividad que proporciona la red residencial como el video bajo demanda, los juegos on-line, el teletrabajo, entre otros, obtengan un desempeño satisfactorio no representa una tarea trivial en absoluto. La estrategia de incrementar el ancho de banda de la conexión a Internet o priorizar un tráfico frente a otro, no logran proporcionar una solución definitiva al problema del desempeño pobre de las aplicaciones y, sobre todo, no garantizan que en un futuro cercano seguirán teniendo algún grado de efectividad. En este contexto, la arquitectura de gestión centrada en el usuario propuesta en el capítulo anterior ha demostrado que puede mejorar las funcionalidades de conectividad que proporciona la red residencial aprovechando los paradigmas SDN y NFV. No obstante, para explotar todo el potencial de que ofrecen ambos paradigmas es necesario implementar un modelo de trabajo colaborativo que permita complementar las aportaciones individuales de cada tecnología. De esta manera, en este capítulo se propone que la red residencial sea capaz de aplicar un tratamiento de tráfico diferenciado con el objetivo de proporcionar las condiciones necesarias para que las aplicaciones que hacen uso de sus servicios de red se desempeñen de forma adecuada. Adicionalmente, se explora la posibilidad de aprovechar los beneficios de una Red de Acceso Óptica Definida por Software (SDOAN) para alcanzar una flexibilidad multinivel que permita proporcionar la Red Residencial como Servicio (RNaaS).

\subsection{Introducción}

El análisis de la arquitectura de la red residencial realizado en la sección 2.1.2, permitió reconocer que la red residencial proporciona servicios de conectividad con soporte de QoS a servicios o aplicaciones de más alto nivel, así como desempeñar la función de extender la red de acceso. En este contexto, la arquitectura de gestión centrada en el usuario propuesta permite enriquecer o ampliar el catálogo de servicios de conectividad que ofrece la red residencial al aprovechar el control de grano fino del tráfico que proporciona un RGW basado en SDN conjuntamente con la posibilidad de incluir nuevas funcionalidades mediante el uso de NFV. No obstante, los componentes SDN y NFV de vMANDO deben trabajar de forma colaborativa para cumplir con los requisitos de conectividad y procesamiento de tráfico que imponen determinadas aplicaciones que se ejecutan en la red residencial, tal y como se explicó en el análisis de las propuestas realizado en la sección 3.5. Un trabajo colaborativo implica que, por una parte, el componente NFV se encarga de desplegar VNFs especializadas y la creación inherente de SFCs permite proporcionar un tratamiento específico a un determinado tipo de tráfico que se origina en la red residencial. Por otra parte, el componente SDN se encarga de clasificar e identificar el tráfico de la red residencial que requiere un tratamiento en particular con el objetivo de entregarlo a la SFC adecuada en vMANDO. 
La arquitectura de gestión propuesta en el capítulo anterior describe la forma en la cual se puede mejorar la usabilidad de la red, hacer frente a la heterogeneidad del entorno y enriquecer el servicio de conectividad de red provisto al permitir y facilitar la implementación del enfoque centrado en el usuario. Para este fin, se definió en detalle un modelo operacional para las distintas capas y sus correspondientes bloques funcionales. Además, se asumió que en el componente NFV de vMANDO deben desplegarse determinadas VNFs encargadas de proporcionar conectividad a Internet y otros servicios a las aplicaciones multimedia que se ejecutan en la red residencial. Sin embargo, para conseguir el modo de trabajo colaborativo descrito previamente se requiere que desde la arquitectura de gestión se coordinen los procesos de despliegue del componente NFV, así como la definición de flujos de tráfico en el componente SDN de forma coherente, es decir, cualquier modificación en el componente NFV debe reflejarse en el componente SDN y viceversa. De esta manera, es necesario que la arquitectura de gestión propuesta también permita que el usuario participe activamente en los procesos de despliegue del componente NFV.

En este contexto, este capítulo aborda el requerimiento de definir un modelo de interacción entre la Arquitectura de Gestión Centrada en el Usuario y la plataforma de NFV que permita orquestar conjuntamente los componentes SDN y NFV para que la red residencial sea capaz de proporcionar un tratamiento de tráfico diferenciado con soporte de QoS. En el modelo de interacción propuesto, una aplicación RENEMA debe acceder a las interfaces NFV-MANO para orquestar el despliegue de VNFs y la creación de SFCs. Además, la aplicación también debe coordinar la creación de flujos de tráfico en el RGW basado en SDN para dirigirlos a la SFC correspondiente encargada de procesarlos. De esta manera, el modelo de interacción descrito requiere la definición del conjunto de funciones que proporcionará la aplicación a través de su API correspondiente con base en el concepto de tratamiento de tráfico diferenciado y las operaciones del ciclo de vida de los distintos elementos de información que proporcionan las interfaces MANO.

Por otra parte, considerando la conclusión obtenida en el capítulo del estado del arte con respecto a los beneficios que proporciona una Red de Acceso Definida por Software (SDAN) en la creación ágil de servicios adaptados a los requerimientos del usuario, en este capítulo también se explora la posible interacción y cooperación entre la arquitectura de gestión y el sistema de control de la SDAN para complementar los servicios de conectividad con soporte de QoS que proporciona una red residencial basada en SDN y NFV. Este enfoque permitiría que el usuario, a través de una aplicación RENEMA, defina y posteriormente modifique las características de la conexión a Internet proporcionada por la red de acceso tales como el ancho de banda mínimo garantizado o la tasa máxima de pérdida de paquetes. Además, un modelo colaborativo entre los sistemas de control de la red residencial y de la red de acceso permitiría implementar mecanismos orientados a optimizar la asignación de recursos en la red de acceso.

Ciertamente, al facilitar que la arquitectura de gestión adicionalmente interactúe con NFV-MANO y el sistema de control de la SDAN se permite que el usuario controle desde una ubicación central todos los componentes y, por lo tanto, los recursos involucrados en la provisión del servicio de red residencial: el $R G W$ basado en SDN, la SDAN y diferentes SFCs. Por otra parte, este enfoque al proporcionar una flexibilidad multinivel también permite que el ISP sea capaz de alcanzar un alto grado de automatización en la puesta en marcha de todos los componentes de la red residencial. De esta manera, al integrar todos los sistemas de control en la infraestructura de computación en la Nube del ISP, la Red Residencial podría ser proporcionada como Servicio (RNaaS).

\subsection{Orquestación Conjunta de SDN y NFV}

Con el objetivo de proporcionar un tratamiento de tráfico diferenciado con soporte de QoS, es necesario que la arquitectura de gestión centrada en el usuario permita orquestar los componentes SDN y NFV para hacerlos trabajar de forma conjunta de tal forma que sus aportaciones individuales sean complementadas entre sí.

En este contexto, la Figura 4.1 (a) muestra el enfoque propuesto en el cual se distinguen dos componentes fundamentales. Por una parte, todas las entidades lógicas que determinan el comportamiento de los elementos físicos y virtuales que forman parte de la red residencial son agrupados 
en el componente de Gestión y Control. Por otra parte, los elementos físicos y virtuales que se encargan de implementar el tratamiento de tráfico diferenciado con base en las indicaciones que proporciona el componente de Gestión y Control representan los recursos de red y computación. Como se puede observar en la Figura 4.1, el Sistema de Gestión permite que el usuario a través de una aplicación RENEMA sea capaz no sólo de controlar el RGW basado en SDN sino también de controlar los recursos de la Red de Acceso Óptica Definida por Software (SDOAN) y crear SFCs personalizadas para que la red residencial sea capaz de proporcionar un tratamiento de tráfico diferenciado. Adicionalmente, es importante recordar que vMANDO concentra dos tipos de tráfico: de gestión y datos como se muestra en la Figura 4.1 (b). De esta manera, el componente de redes (Networking Component) es el encargado de aplicar un tratamiento de tráfico diferenciado en el tráfico de datos.

A continuación, se presenta una descripción más detallada de los componentes antes mencionados.

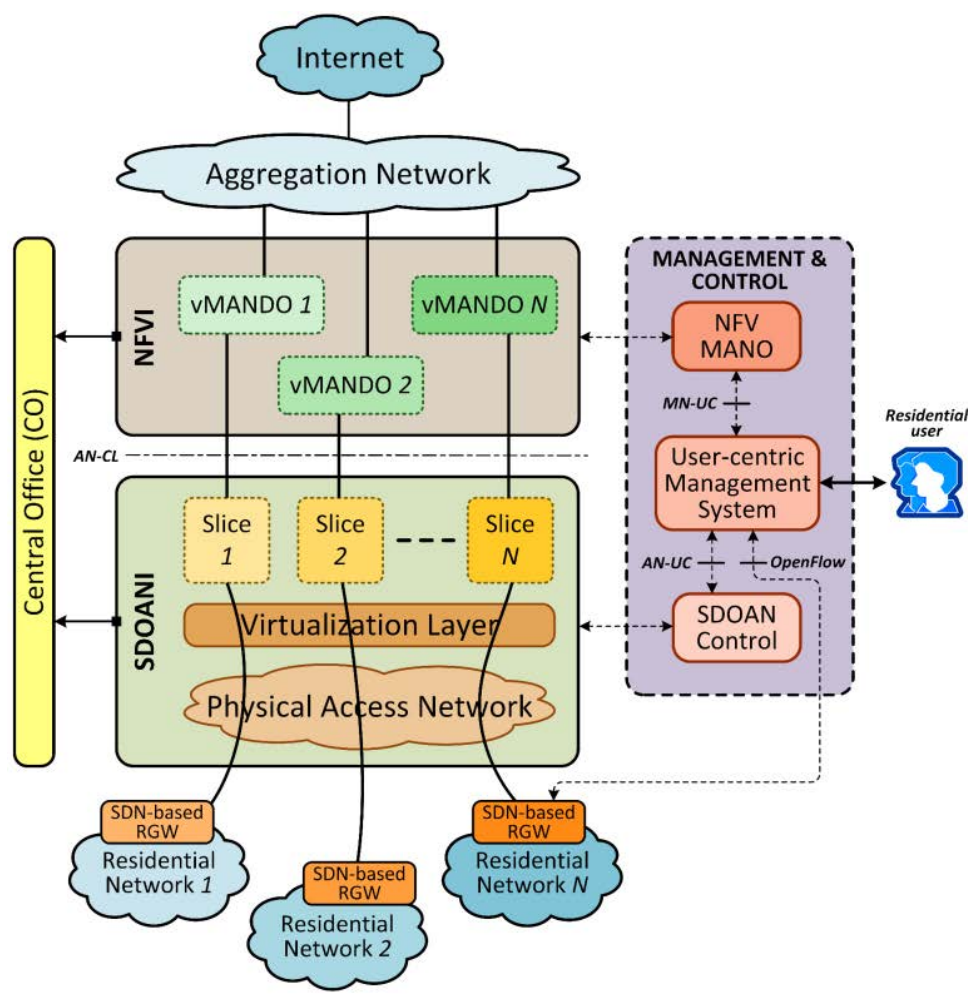

(a)

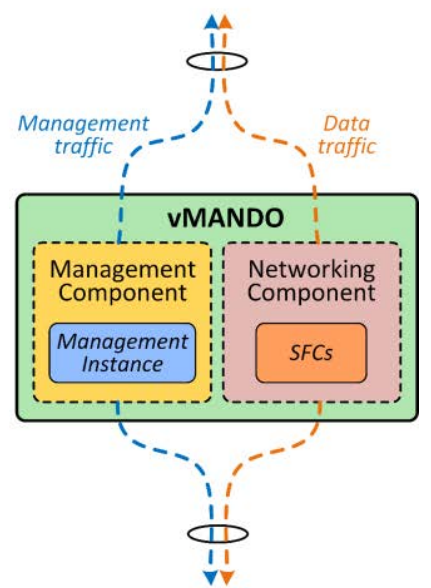

(b)

Figura 4.1 Orquestación conjunta de SDN y NFV: (a) El sistema de gestión interactúa con el sistema de control de la Red de Acceso Óptica Definida por Software y con la Gestión y Orquestación de NFV para coordinar el funcionamiento de los componentes SDN y NFV. (b) Componentes de Gestión y Red que forman parte de vMANDO.

\subsubsection{Gestión y Control}

Este componente está formado por NFV-MANO, el Sistema de Gestión Centrado en el Usuario (Usercentric Management System) y el Sistema de Control de la SDOAN (SDOAN Control). Este componente tiene por objetivo definir las características funcionales o de comportamiento de los elementos físicos y virtuales que forman parte de la red residencial con base en los requerimientos del usuario. Para conseguir esta funcionalidad, tanto NFV-MANO como el sistema de control de la SDOAN proporcionan un conjunto de Interfaces de Programación de Aplicaciones (APIs). Para el caso de NFV, como se explicó en la sección 2.3.5, las interfaces MANO permiten desplegar las distintas VNFs, SFCs y redes virtuales requeridas en la composición de la estructura interna de cada vMANDO. Para el caso de la SDOAN, las interfaces que proporciona el sistema de control permiten definir las características de la red de acceso tales como los niveles de ancho de banda mínimo garantizado tanto en los canales ascendente y descendente, latencia, jitter, tasa de perdida de paquetes, priorización del tráfico y 
clasificación. De esta manera, las opciones de configuración de cada recurso de red están disponibles a través de diferentes aplicaciones RENEMA que el sistema de gestión incorpora para que el usuario las utilice.

Se han definido puntos de referencia para que el Sistema de Gestión interactúe con los demás bloques de control. Así, el punto de referencia AN-UC (Access Network - User-Centric Management System) está destinado para la comunicación con el Sistema de Control de la SDOAN; mientras que el punto de referencia $M N-U C$ (MANO - User-Centric Management System) está destinado para la comunicación con el sistema NFV-MANO.

\subsubsection{Recursos de Red y Computación}

El RGW basado en SDN, la infraestructura de la SDOAN (SDOANI) y la infraestructura de NFV (NFVI) representan los recursos de red y computación a ser controlados por el componente de Gestión y Control. El RGW basado en SDN permite definir flujos de tráfico específicos a los cuáles un determinado conjunto de VNFs les aplicarán un tratamiento diferenciado en la NFVI. El Sistema de Gestión Centrado en el Usuario define los flujos de tráfico mediante OpenFlow.

Considerando que el nodo de acceso, en este caso la OLT, pudiese incorporar capacidades de virtualización, se estaría proporcionando una infraestructura de SDOAN (SDOANI), similar al concepto de NFVI. De esta manera, la capa de virtualización, implementada sobre la red de acceso física subyacente, tiene por objetivo proporcionar una porción virtual personalizable de la red de acceso por red residencial. Una vez que la porción virtual es instanciada, sus parámetros funcionales son gestionados a través del API que el sistema de control de la SDOAN exporta en el punto de referencia $A N-U C$.

En el análisis de las propuestas realizado en la sección 3.5 se explicó la necesidad de considerar los conceptos de Nube de Borde (Edge Cloud) para evitar una sobrecarga en el centro de datos principal del ISP, así como reducir la latencia en los servicios que se ejecutan en la red residencial. La iniciativa CORD es un ejemplo de Nube de Borde que ha transformado la Oficina Central (CO) tradicional en un centro de datos. Siguiendo este enfoque, se coloca una NFVI conjuntamente con la SDOANI en la Oficina Central. En esta infraestructura Cloud se despliega un vMANDO y sus respectivos componentes por red residencial. Como se muestra en la Figura 4.1, cada porción virtual de red de acceso está conectada a un determinado vMANDO a través del punto de referencia $A N-C L$ (Access Network CLoud). Considerando la ubicación física y lógica de los diferentes componentes de SDOANI y NFVI, el punto de referencia $A N-C L$ puede requerir la implementación de redes físicas y/o virtuales para conseguir la funcionalidad esperada.

Es importante mencionar que la porción virtual personalizable de la red de acceso también podría ser proporcionada por la plataforma de NFV de forma nativa. En este caso, la arquitectura de referencia de NFV analizada en la sección 2.3.1 y mostrada en la Figura 2.11, debería incluir en la parte de Hardware de red (Network Hardware) el plano de datos del nodo de acceso u OLT. A su vez, el respectivo plano de control que proporciona las interfaces de control debería ser incluido como un bloque funcional dentro de MANO. En consecuencia, el Sistema de Gestión debería interactuar solamente con la plataforma de NFV a través de único punto de referencia.

Se podría decir que la propuesta de dotar de capacidades de virtualización al nodo de acceso, como se muestra en la Figura 4.1, está alineada con la propuesta de permitir que OLTs tradicionales sean compatibles con los principios de SDN mencionada en la sección 2.4.2. De esta manera, se proporciona un mecanismo transitorio para mejorar la flexibilidad y eficiencia de los nodos de acceso heredados y continuar su uso hasta que finalmente NFV y SDN alcancen un nivel de madurez que permita crear plataformas NFV que nativamente proporcionen porciones virtuales de red de acceso como se mencionó anteriormente. 


\subsection{Tratamiento de Tráfico Diferenciado}

El tratamiento de tráfico diferenciado consiste en aplicar una SFC específica a un determinado tipo de tráfico que pertenece a una aplicación que se ejecuta en la red residencial. Este enfoque proporciona flexibilidad para aplicar diferentes estrategias de procesamiento de tráfico, así como conseguir una Calidad de Servicio (QoS) en particular. Para conseguir esta funcionalidad, se propone que el usuario sea capaz de especificar las aplicaciones, como el video bajo demanda o las descargas P2P, que tendrán un tratamiento específico a través de la definición de flujos de tráfico (traffic flows). Existe la opción de agrupar flujos de tráfico que comparten características similares mediante la definición de contenedores de tráfico (traffic containers). Un contenedor de tráfico representa un contenedor lógico para acomodar uno o varios flujos de tráfico. Considerando que la red residencial incorpora un RGW basado en SDN, la definición de flujos de tráfico puede ser realizada de múltiples maneras. Por ejemplo, en base a los puertos físicos del RGW, direcciones MAC o IP de los dispositivos de usuario o puertos TCP/UDP de las aplicaciones utilizadas.

La Figura 4.2 muestra un ejemplo de definición de diferentes flujos de tráfico, identificados de $A$ a $F$. Los flujos de tráfico con similares características son agrupados mediante la definición de contenedores de tráfico, identificados de 1 a $n$. De igual manera, distintas SFCs son creadas e identificadas de 1 a $n$, las cuales están encargadas de recibir el tráfico de cada contenedor y aplicar un tratamiento en particular.

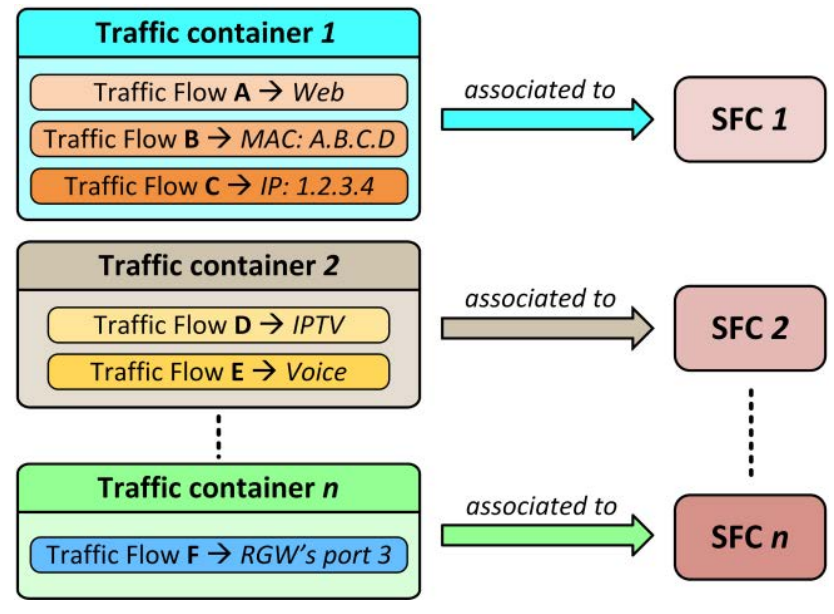

Figura 4.2 Tratamiento de tráfico diferenciado. Se definen diferentes flujos de tráfico a ser agrupados en contenedores de tráfico. Posteriormente, cada contenedor es asociado a una SFC específica de acuerdo con la estrategia de procesamiento de tráfico o el nivel de QoS solicitado.

De acuerdo con la Figura 4.2, se necesita especificar un Id para identificar un contenedor de tráfico específico y su correspondiente SFC que es desplegada en vMANDO. Adicionalmente, se utiliza también este Id para definir un canal virtual encargado de transportar y dirigir correctamente los flujos de tráfico pertenecientes a un contenedor de tráfico dado desde la red residencial a la SFC. Se pueden utilizar diferentes opciones para diferenciar los contenedores de tráfico, por ejemplo: VLANs, VXLAN o túneles. Con el objetivo de explicar de mejor manera la propuesta, se adopta un enfoque basado en VLANs, como el que mencionan Kim et al. [106], para crear los contenedores.

De esta manera, se pretende etiquetar doblemente el tráfico de la red residencial utilizando el estándar 802.1ad (Q-in-Q). Se utiliza la etiqueta externa $S$ para identificar a la red residencial y se utiliza la etiqueta interna $C$ para identificar el contenedor de tráfico. Por ejemplo, el usuario podría definir varios contenedores para asociar cada uno a un flujo de tráfico específico siguiendo una relación 1:1 como se muestra en la Figura 4.3. Es importante mencionar que CORD también adopta el mecanismo Q-in-Q, sin embargo, debido a que no se considera el enfoque de múltiples SFCs por red residencial, su implementación es distinta. De esta manera, se utiliza la etiqueta $S$ para identificar la máquina virtual donde reside el Gateway del Suscriptor virtual (vSG), mientras que la etiqueta $C$ se utiliza para identificar al vSG. 


\subsubsection{Estructura Interna de vMANDO para Soportar el Tratamiento de Tráfico Diferenciado}

El tratamiento de tráfico diferenciado a realizarse en vMANDO requiere de una estructura específica compuesta por varias VNFs y redes virtuales. La Figura 4.4 muestra la estructura interna de vMANDO propuesta para tal fin. Es importante recordar que vMANDO está compuesto por el componente de Gestión, representado por la Instancia de Gestión Centrada en el Usuario y, por el componente de Redes, representado por el conjunto de VNFs y las respectivas redes virtuales que las conectan para formar una determinada SFC. Cada vMANDO debe ser conectado a las redes complementarias de Entrega (Delivering), Servicios (Services) y Externa (External) para obtener la funcionalidad requerida como se explicó en la sección 3.3.2. Como se observa, todos los componentes y las redes complementarias son virtuales y su despliegue y gestión están a cargo de NFV-MANO. Como se mencionó anteriormente, el objetivo consiste en que el usuario sea capaz de crear SFCs a través de una aplicación RENEMA, utilizando para ello las interfaces proporcionadas por MANO.
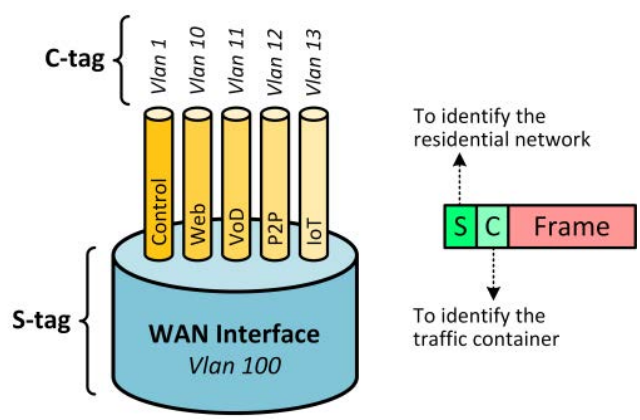

Figura 4.3 Por medio del uso de etiquetas VLAN, se divide la interfaz WAN de la red residencial en varios canales virtuales que permiten transportar y dirigir los flujos de tráfico a su correspondiente SFC.

Considerando que el tráfico de la red residencial está doblemente etiquetado, la Red de Entrega (Delivering Network) direcciona el tráfico de una red residencial dada a su correspondiente vMANDO mediante el uso de la etiqueta $S$ como se muestra en la Figura 4.4. Una vez allí, el tráfico de la red residencial requiere dos niveles de clasificación debido a que se implementa un control en banda. La Red de Clasificación (Classification Network) representa el primer nivel y se encarga de clasificar el tráfico de control y el tráfico de datos. La VNF de Clasificación \& Conformación (Classification \& Shaping) representa el segundo nivel y está destinada a colocar los flujos de tráfico que pertenecen a un contenedor de tráfico en el canal virtual correspondiente. Es importante aclarar que la VNF de Clasificación y Conformación no representa a la función de clasificación de servicio definida por el IETF en la RFC 7665 [41] la cual se utiliza para clasificar el tráfico y dirigirlo por el conjunto de VNFs que forman parte de una determinada SFC. Como se explicará en el capítulo 5, la función de clasificación de servicio de la SFC utiliza una política de clasificación la cual se especifica en el descriptor de VNFFG correspondiente.

Las funciones de clasificación y direccionamiento, que realizan las redes de Clasificación y Entrega respectivamente, podrían ser implementadas en una sola red. No obstante, el mecanismo de identificación utilizado tendría un significado global, es decir, el espacio de identificación total disponible para los contenedores de tráfico debe ser compartido por todas las redes residenciales dentro del dominio del ISP. En el caso de utilizar VLANs, los 12 bits del campo VLAN ID para la etiqueta $C$ representa un valor limitado de Ids disponibles para ser utilizado por todos los clientes del ISP. Por otra parte, un enfoque de red única requiere un mecanismo efectivo que implemente las funciones de clasificación y direccionamiento de los flujos de tráfico pertenecientes a los diferentes contenedores definidos por red residencial, lo cual podría resultar en una tarea compleja debido a problemas de seguridad y privacidad.

Con respecto al despliegue de vMANDO, las VNFs que lo componen se clasifican en dos tipos. En primer lugar, están las VNFs fijas necesarias para proporcionar un servicio básico o por defecto y que son obligatoriamente desplegadas por red residencial. Este tipo está representado por las VNFs 
Encaminamiento + NAT (Routing + NAT) y Clasificación \& Conformación. La primera se corresponde con las funciones básicas proporcionadas por un RGW tradicional. La segunda emplea las funciones de limitación de velocidad y clasificación de tráfico para crear el número requerido de terminaciones de canal virtual. Este proceso es necesario debido a que se divide la interfaz WAN del RGW basado en SDN en varios canales virtuales destinados a transportar y dirigir el tráfico de un contenedor a su correspondiente SFC. Como se verá más adelante en la sección de APIs, la creación de un contenedor de tráfico requiere la especificación de un ancho de banda, el cuál es utilizado para crear el canal virtual correspondiente.

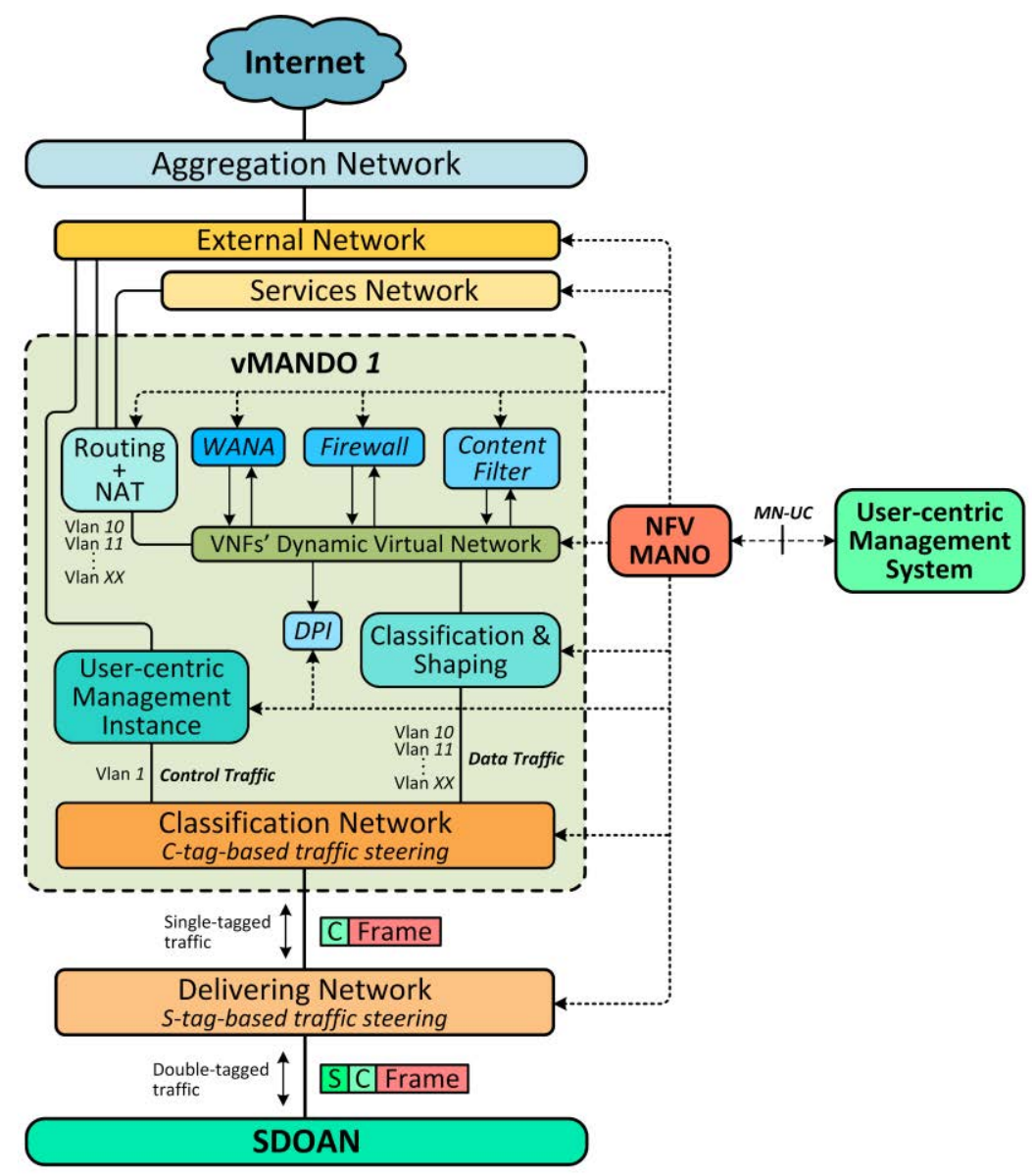

Figura 4.4 Estructura interna de vMANDO. La Gestión y Orquestación (MANO) de NFV está a cargo de instanciar diferentes VNFs para crear una SFC específica de acuerdo con las especificaciones recibidas desde el Sistema de Gestión Centrado en el Usuario.

En segundo lugar, están las VNFs adicionales que son agregadas bajo demanda con el propósito de crear SFCs específicas, complementando de esta manera el conjunto de VNFs que componen cada vMANDO. Este enfoque proporciona una gran flexibilidad para aplicar diferentes estrategias de tratamiento basado en SFCs a un contenedor de tráfico dado, enriqueciendo el servicio de conectividad de red con soporte de QoS provisto por la SDOAN y la red residencial basada en SDN. Por ejemplo, el usuario podría necesitar aplicar un tratamiento diferenciado para el tráfico de Torrent y Web para lo cual define, en primer lugar, los flujos de tráfico y, en segundo lugar, los contenedores asociados como se muestra en la Figura 4.5. Posteriormente, cada contenedor de tráfico será asociado a una SFC específica encargada de aplicar un tratamiento en particular. Dichas SFCs están compuestas por las VNFs fijas y las VNFs que el usuario agrega con base en el tratamiento o funcionalidad que desea implementar. En el caso de la Figura 4.5, se asigna un acelerador WAN (WANA) al contenedor 1 (Torrent) para definir la SFC $A$, mientras que se asigna un filtro de contenidos (Content Filter) y un cortafuegos (Firewall) al contenedor 2 (Web) para definir la SFC $B$. Mediante este enfoque, el usuario puede agregar complejas VNFs como 
un IDS (Intrusion Detection System) con el objetivo de reconocer patrones de tráfico sospechosos y proteger los datos sensibles de los usuarios de la red residencial.

En esta nueva configuración de red propuesta, el $R G W$ basado en $S D N$, la $S D O A N$ y la VNF Clasificación \& Conformación forman un mecanismo de tres niveles que permite definir políticas de conformado de tráfico para el conjunto de canales virtuales creados entre la red residencial y la red de agregación. Cada nivel permite la definición de colas tanto para el tráfico ascendente como descendente. Estas políticas están destinadas a mejorar el nivel de QoS del tráfico de las diferentes aplicaciones que se ejecutan en la red residencial, así como de los servicios específicos proporcionados por el ISP tales como VoD (Video On demand), IPTV, voz, etc.

Por ejemplo, si el usuario residencial desea observar un espectáculo determinado de TV con la máxima calidad posible, podría especificar un nivel de QoS apropiado a través del sistema de gestión. Para cumplir con este requisito, se deben modificar las políticas de conformado de tráfico en los tres niveles. Si la calidad resultante no es la esperada, la SDOAN también podría implementar un enfoque de contratación de ancho de banda bajo demanda $(\mathrm{BoD})$. De esta manera, el usuario especifica el valor de ancho de banda adicional y, dependiendo de la periodicidad del evento, podría también especificar un horario. Todo este proceso se realiza mediante una aplicación RENEMA que accede al API proporcionado por el sistema de control de la SDOAN.

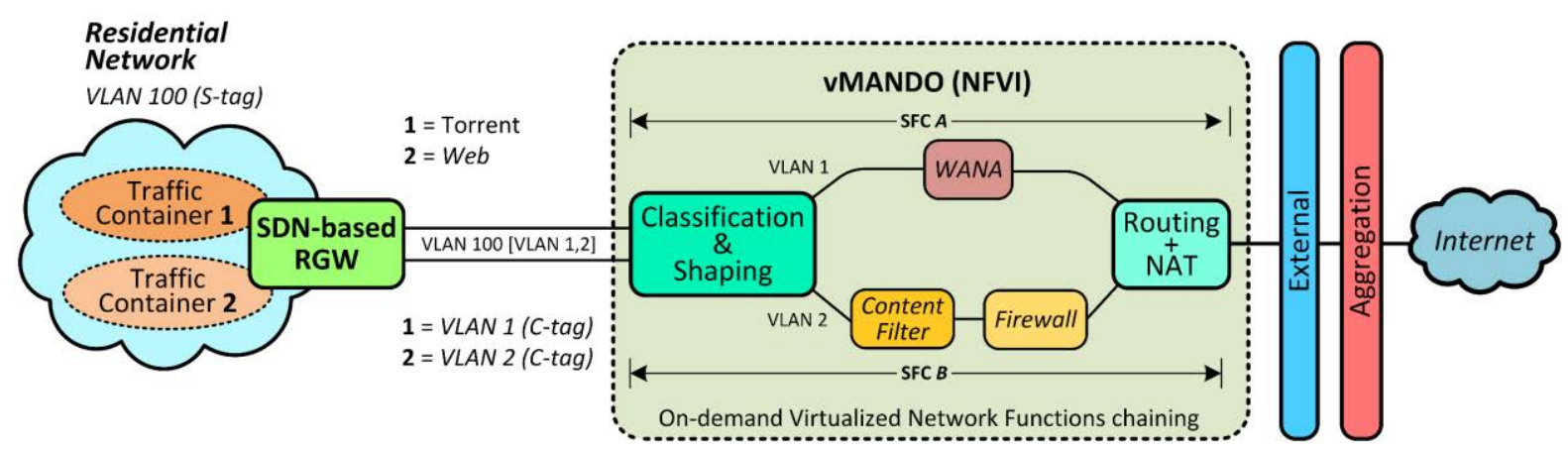

Figura 4.5 Ejemplo de tratamiento de tráfico diferenciado con calidad de servicio (QoS). Las SFCs $A$ y $B$ son asignadas a los contenedores de tráfico 1 y 2 respectivamente.

\subsection{Aplicación RENEMA para el Tratamiento de Tráfico Diferenciado}

En el contexto de la Arquitectura de Gestión Centrada en el Usuario propuesta, una aplicación RENEMA debe proporcionar las funcionalidades de tratamiento de tráfico diferenciado mencionadas en las secciones anteriores. De forma general, la aplicación permite crear flujos de tráfico y contenedores de tráfico para posteriormente asignar un flujo de tráfico a un contenedor en particular. Por otra parte, el proceso de creación de una SFC involucra la especificación de las VNFs que formarán parte de ella y la posterior asociación al contenedor de tráfico previamente creado.

Para conseguir este modo de operación, se define la aplicación de Tratamiento de Tráfico (Traffic Treatment), la cual incorpora dos bloques funcionales. Un primer bloque se encarga de controlar las operaciones CRUD (Create, Read, Update and Delete) de contenedores y flujos de tráfico denominado como Traffic Container Handler. Un segundo bloque se encarga de controlar las operaciones CRUD de SFCs y VNFs constituyentes denominado como SFC Handler. La Figura 4.6 muestra en detalle los bloques funcionales y la interacción con NFV-MANO y la Arquitectura de Gestión.

\subsubsection{Traffic Container Handler}

Como se analizó en la sección 4.3, el tratamiento de tráfico diferenciado requiere la definición de flujos de tráfico. Si existen flujos que comparten características similares pueden ser agrupados en un contenedor de tráfico. Con base en el mecanismo utilizado para identificar dicho contenedor, éste bloque funcional tiene como objetivo coordinar la creación del contenedor en el RGW basado en SDN y del 
canal virtual que conectará el contenedor con su correspondiente SFC en la NFVI. Posterior a la creación de flujos de tráfico y su respectivo contenedor, este bloque funcional también está destinado a controlar el resto de operaciones CRUD asociadas. De esta manera, se puede actualizar, eliminar o consultar los contenedores y los flujos de tráfico creados.

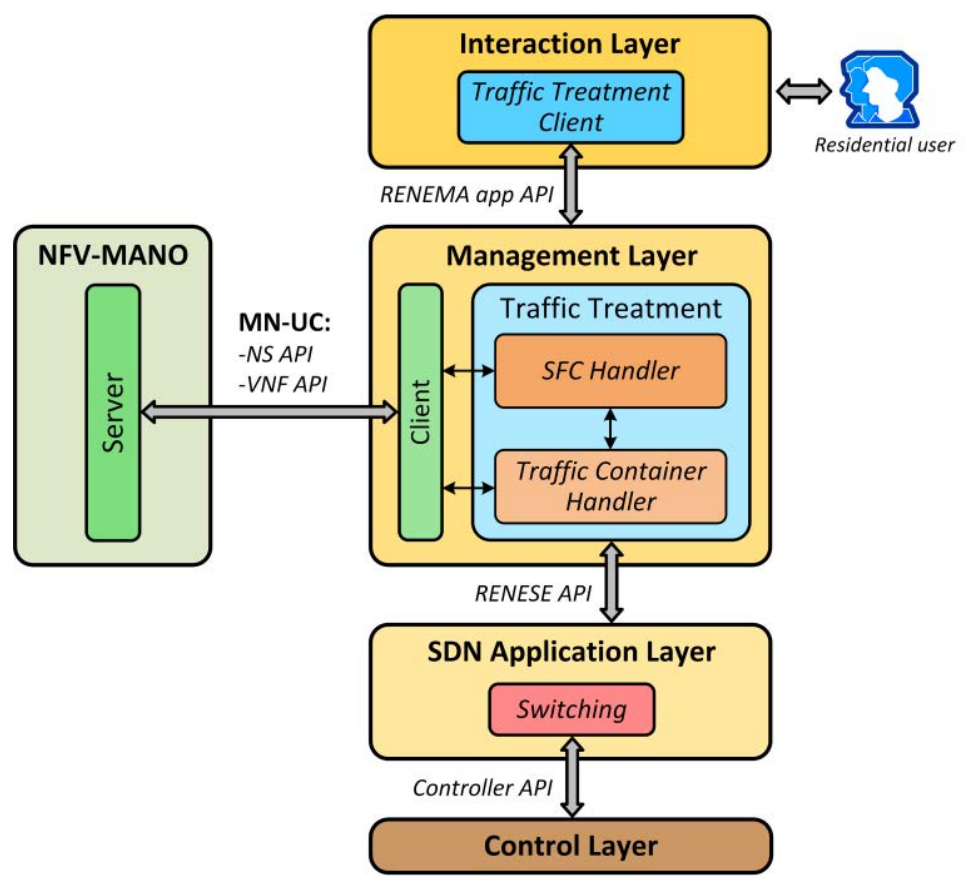

Figura 4.6 Aplicación de Tratamiento de Tráfico. Los bloques funcionales de la aplicación utilizan las APIs proporcionadas por NFV-MANO para controlar los procesos CRUD de SFCs en la NFVI y el protocolo RENESE para controlar los procesos CRUD de contenedores de tráfico en el RGW basado en SDN.

Con respecto a la creación de contenedores de tráfico, es importante mencionar que, al momento de desplegar una nueva red residencial, ésta es provista con dos contenedores por defecto, uno asociado al tráfico de control interno (flujo por defecto) y otro asociado al tráfico de datos (flujo por defecto) manteniendo una relación 1:1. De esta manera, la aplicación tiene por objetivo guiar al usuario en la creación de nuevos flujos de tráfico y contenedores y la respectiva asignación de flujos a contenedores. Además, la aplicación permite personalizar la configuración del contenedor creado por defecto para el tráfico de datos. Por ejemplo, la aplicación indicaría al usuario la posibilidad de mejorar la calidad de servicio o calidad de experiencia mediante la clasificación del tráfico presente en la red y la posterior aplicación de servicios de tratamiento de tráfico especializados sobre la clasificación realizada.

Considerando el uso de VLANs como mecanismo de identificación, el Traffic Container Handler debe instruir sobre cómo etiquetar el tráfico al servicio de Conmutación que se ejecuta en la capa de Aplicación SDN con base en los contenedores de tráfico previamente creados, utilizando para ello el protocolo RENESE. El etiquetado que realizaría el servicio de Conmutación puede ser simple o doble, y dependerá de las políticas del ISP. Por ejemplo, en un caso, el ISP puede especificar que la etiqueta externa $S$ sea colocada por la ONT y la etiqueta interna $C$ por el servicio de Conmutación. En otro caso, el propio servicio de Conmutación puede colocar las dos etiquetas.

Adicionalmente, la creación de un nuevo contenedor de tráfico en la red residencial involucra incluir el Id de VLAN en la Red de Clasificación para actualizar el proceso de clasificación y direccionamiento de tráfico, así como la creación de la terminación del canal virtual en la VNF de Clasificación \& Conformación. La interacción entre el Traffic Container Handler y la plataforma de NFV se realiza mediante el punto de referencia $M N-U C$, específicamente se utilizan las interfaces NS y VNF proporcionadas por MANO como se muestra en la Figura 4.6. 


\subsubsection{SFC Handler}

Éste bloque funcional está encargado de coordinar la creación de una SFC específica y aplicarla a un determinado contenedor de tráfico. Para este fin, como se explicó en la sección 2.3.4, se debe crear el correspondiente descriptor denominado como VNFFGD. El proceso de creación de este descriptor involucra obtener determinados parámetros funcionales tanto de descriptores de VNF como de contenedores de tráfico.

En VNFFGD, se especifican las VNFs que formarán parte de la SFC. Estas VNFs representan nuevas funcionalidades a integrar en la red residencial y son previamente seleccionadas por el usuario desde la aplicación de Tratamiento de Tráfico. Por otra parte, es necesario vincular un contenedor de tráfico a una determinada SFC y especificar una política de clasificación en el VNFFGD que le permita a la función de clasificación de servicio de la SFC determinar la ruta específica que debe seguir el tráfico a través del conjunto de VNFs desplegadas en vMANDO. En el caso de utilizar VLANs, el valor de la etiqueta $C$ que identifica al contenedor de tráfico, debería ser utilizado como política de clasificación en el VNFFGD y, por lo tanto, también permitiría establecer el vínculo entre el contenedor de tráfico y la SFC.

Una vez que se obtiene un VNFFGD válido, el SFC Handler debe incorporarlo al catálogo de Servicios de Red de la plataforma de NFV para posteriormente crear la instancia correspondiente. Cuando la instancia de SFC está activa, éste bloque funcional también se encarga de las operaciones CRUD respectivas. Así, es posible actualizar, eliminar o consultar las SFCs definidas en el Sistema de Gestión integral de la red residencial. Al igual que el Traffic Container Handler, las interfaces NFV-MANO en el punto de referencia $M N-U C$ son utilizadas para proporcionar las funcionalidades descritas como se observa en la Figura 4.6.

Con respecto a la creación de SFCs, es importante considerar los diferentes tipos de tráfico presentes en vMANDO ya que se necesita crear una SFC específica para cada uno de ellos. Además del tráfico de control interno y del de datos de usuario, existe el tráfico destinado a los servicios comunes y el tráfico de control externo. Al momento de desplegar una nueva red residencial, se crean SFCs por defecto asociadas a los tipos de tráfico mencionados previamente. De esta manera, la aplicación de Tratamiento de Tráfico actúa sobre el tráfico de datos, permitiendo la creación de nuevas SFCs que serán aplicadas a los flujos de tráfico pertenecientes a un contenedor dado o permitiendo la modificación de la SFC asignada por defecto. A continuación, se definen los siguientes tipos de SFCs a ser especificadas por diferentes VNFFGDs:

a. SFC por defecto para el tráfico de datos. En ausencia de SFCs o VNFFGs definidas por el usuario residencial, la SFC por defecto permite dirigir el tráfico de datos de la red hacia Internet. El descriptor correspondiente debe incluir solamente las VNFs fijas que forman parte de la SFC. Se utilizan las capacidades de envío proporcionadas por cada VNF para dirigir el tráfico por las distintas VNFs que forman parte de la SFC.

b. SFC para un determinado tratamiento de tráfico. Una vez que se ha especificado un nuevo esquema de tratamiento a ser aplicado a un contenedor de tráfico, se crea la correspondiente SFC que establece la ruta que debe seguir el tráfico a través de las VNFs involucradas.

c. SFC para el tráfico de control interno. Considerando que se utiliza un control en banda, se define una SFC para direccionar el tráfico de control entre la VNF del Sistema de Gestión y el RGW basado en SDN.

d. SFC para el tráfico de control externo. Teniendo en cuenta que los clientes de las aplicaciones RENEMA deben conectarse con su respectivo back-end desplegado en vMANDO, se requiere de una SFC para dirigir el tráfico de control externo (Internet) hacia la VNF del Sistema de Gestión.

e. SFC para el tráfico destinado a la red de servicios. En la definición de vMANDO se incluye la Red de Servicios con el objetivo de proporcionar conectividad entre la red residencial y servicios de red comunes como DHCP o DNS. De esta manera, se requiere una SFC para dirigir el tráfico de la red residencial que necesita alcanzar este tipo de servicios comunes. 


\subsubsection{Cliente de NFV-MANO}

Como se ha mencionado anteriormente, la aplicación de Tratamiento de Tráfico debe interactuar con las interfaces proporcionadas por NFV-MANO en el punto de referencia $M N-U C$ para coordinar la creación de SFCs y asignarlas a determinados contenedores de tráfico. Estas interfaces o APIs son implementadas por una plataforma de NFV siguiendo las recomendaciones de ETSI. Considerando que cada plataforma define la estructura, método de acceso, autenticación, autorización y funciones o servicios a ser proporcionados por las interfaces, es conveniente incorporar un cliente que se adapte a dichas características específicas. Por ejemplo, en la sección 2.3.6, se mostraron las interfaces MANO que proporciona Tacker denominadas como NFVO NS API, VNFM API y VNFFG API. El acceso a este conjunto de APIs se realiza a través de un cliente el cual se encarga de gestionar la comunicación con el respectivo servidor.

Como se observa en la Figura 4.6, el cliente NFV-MANO está disponible a nivel de la Capa de Gestión con lo cual su uso no está limitado a la aplicación de Tratamiento de Tráfico. De esta manera, otras aplicaciones podrían utilizar este cliente para acceder a las interfaces NFV-MANO con el objetivo de proporcionar funcionalidades complementarias como, por ejemplo, la monitorización de componentes NFV desplegados en vMANDO.

\subsubsection{Objetos y API de la Aplicación}

Con el fin de proporcionar la funcionalidad de tratamiento de tráfico diferenciado descrita en este capítulo, la aplicación correspondiente incorpora varias operaciones destinadas a actuar sobre flujos de tráfico, contenedores de tráfico, VNFs y SFCs. Al igual que el resto de aplicaciones, las operaciones son expuestas desde la Capa de Gestión a través de un API REST para que el cliente correspondiente las pueda utilizar. El funcionamiento de la aplicación está determinado por los diagramas de estado definidos en la sección 3.4, de esta manera la aplicación debe alcanzar el estado Activo para procesar solicitudes REST y mensajes RENESE.

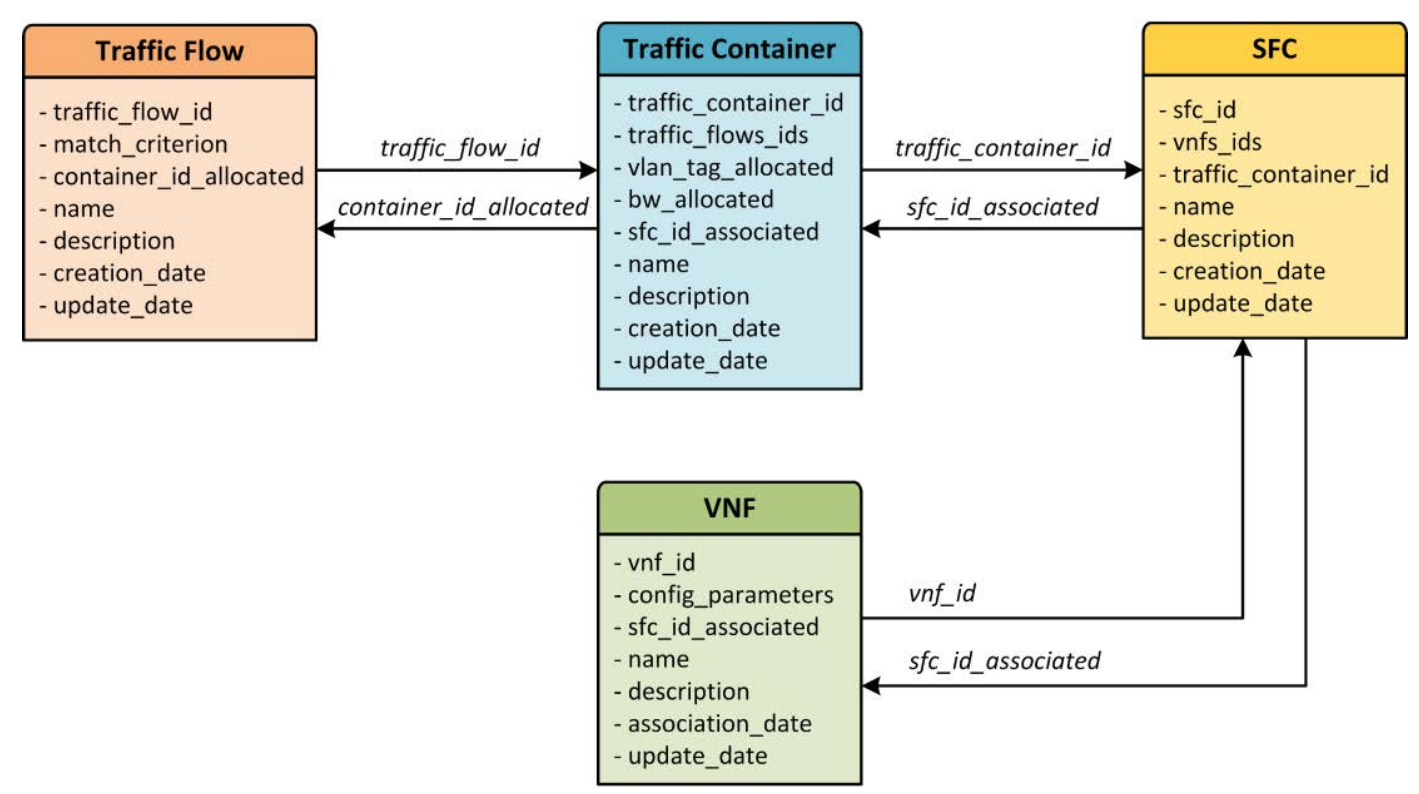

Figura 4.7 Objetos definidos para el tratamiento de tráfico diferenciado y la relación existente entre los mismos.

La aplicación utiliza objetos para representar flujos de tráfico, contenedores de tráfico, VNFs y SFCs. Se definen un conjunto de atributos para cada objeto como se muestra en la Figura 4.7. Los valores de los atributos pueden ser asignados por el usuario residencial o por la propia aplicación al momento de utilizar el API. Por ejemplo, los valores de identificación, fecha de creación o fecha de actualización son asignados por la aplicación, mientras que los valores para nombre o descripción son asignados por el usuario. La Figura 4.7 también muestra que la creación de un determinado objeto está condicionada 
por la creación previa de otro. Por ejemplo, para crear una SFC es necesario crear previamente un contenedor de tráfico y disponer de descriptores válidos en el catálogo de VNFs. A su vez, para crear el contenedor es necesario crear previamente un flujo de tráfico. En el proceso de creación de la SFC se debe especificar los Ids de las VNFs constituyentes y el Id del contenedor de tráfico a ser asociado.

Por otra parte, en respuesta a la eliminación de objetos que están siendo utilizados por otros se podría considerar dos opciones. En primer lugar, no permitir la eliminación de un objeto determinado. Por ejemplo, si se solicita eliminar un contenedor de tráfico que está siendo utilizado por una SFC, la aplicación indicaría que la operación no puede ser realizada. En segundo lugar, permitir la eliminación del objeto seleccionado lo cual eliminaría también los objetos asociados. Por ejemplo, si se solicita eliminar un flujo de tráfico, el sistema eliminaría tanto el contenedor de tráfico como la SFC y el tráfico afectado sería reasignado al flujo y contenedor por defecto (tráfico de datos).

Como se mencionó anteriormente, los bloques funcionales de la aplicación de Tratamiento de Tráfico están encargados de controlar las operaciones CRUD de flujos de tráfico, contenedores, VNFs y SFCs. Estas operaciones se incorporan en la correspondiente API REST de la aplicación. De forma general, el API incluye cinco operaciones elementales que se pueden aplicar a los distintos objetos que manipula la aplicación más una operación exclusiva para VNFs como se observa en la Tabla 4.1.

Tabla 4.1 API REST proporcionada por la aplicación de Tratamiento de Tráfico.

\begin{tabular}{|c|c|c|}
\hline $\begin{array}{l}\text { Operaciones del } \\
\text { API }\end{array}$ & Descripción & Aplicabilidad \\
\hline List & Lists the objects created & $\begin{array}{l}\text { - Traffic flows } \\
\text { - Traffic containers } \\
\text { - VNFs } \\
\text { - SFCs }\end{array}$ \\
\hline Show & $\begin{array}{l}\text { Shows detailed information of an object based on the Id } \\
\text { provided }\end{array}$ & $\begin{array}{l}\text { - Traffic flows } \\
\text { - Traffic containers } \\
\text { - VNFs } \\
\text { - SFCs }\end{array}$ \\
\hline Create & Creates a specific object by providing the required parameters & $\begin{array}{l}\text { - Traffic flows } \\
\text { - Traffic containers } \\
\text { - SFCs }\end{array}$ \\
\hline Update & Updates the values of certain attributes of the object & $\begin{array}{l}\text { - Traffic flows } \\
\text { - Traffic containers } \\
\text { - VNFs } \\
\text { - SFCs }\end{array}$ \\
\hline Delete & Deletes an object based on the Id provided & $\begin{array}{l}\text { - Traffic flows } \\
\text { - Traffic containers } \\
\text { - SFCs }\end{array}$ \\
\hline Restore & $\begin{array}{l}\text { Restores the default configuration parameters of an object } \\
\text { based on the Id provided }\end{array}$ & - VNFs \\
\hline
\end{tabular}

Es importante mencionar que el API propuesto permite realizar operaciones de consulta (list - show), actualización de la configuración (update) y restauración de la configuración por defecto (restore) de VNFs considerando que el resto de operaciones como actualización (udpate) y eliminación (delete) son contraladas exclusivamente por el ISP. Por otra parte, la operación crear (create) SFC de la aplicación utiliza de forma implícita la operación actualizar (update) el NS de MANO. El API propuesta está basada en la definición que realiza ETSI sobre las operaciones que debe proporcionar NFV-MANO a través de las interfaces NS y VNF como se explicó en la sección 2.3.5. De esta manera, la aplicación de Tratamiento de Tráfico utiliza las interfaces orientadas a descriptores y posteriores instancias de NS, VNF y VNFFG para proporcionar la funcionalidad requerida. 
En el capítulo 5, se realiza una definición más detallada del API REST en la cual se incluyen URIs y métodos HTTP utilizados. También se proporcionan tablas complementarias que definen los parámetros de solicitud y respuesta del API REST para las operaciones CRUD de los diferentes objetos que controla la aplicación.

\subsection{Detalles Operacionales}

Con el objetivo de explicar de mejor manera el concepto de tratamiento de tráfico diferenciado, se explica en detalle el proceso a seguir y la interacción entre la aplicación, el servicio de Conmutación y $N F V-M A N O$. Se considera que el proceso de Inicio se ha realizado de forma satisfactoria y la aplicación se encuentra en estado Activo. Por otra parte, es importante destacar que vMANDO en el contexto de NFV representa un servicio de red (NS) y, por lo tanto, se utiliza el correspondiente descriptor para crear una instancia. En el capítulo 5, se presenta en detalle el proceso de implementación de vMANDO como un servicio de red. No obstante, en esta sección es importante considerar que el proceso de instanciación de vMANDO se ha realizado con éxito y que todos sus componentes se encuentran funcionando correctamente. Además, es importante tener presente que agregar nuevas VNFs debido a la creación de nuevas SFCs implica la actualización de vMANDO y, por lo tanto, utilizar la operación de actualización del NS. El proceso de aplicar un tratamiento de tráfico diferenciado a un determinado contenedor de tráfico puede resumirse en cinco pasos fundamentales como se muestra en la Figura 4.8.

- En el primer paso, se crean los flujos de tráfico (Create traffic flow) que formarán parte del contenedor utilizando para ello el API correspondiente y proporcionando los parámetros requeridos. El número de flujos a crear depende de las necesidades del usuario. Por ejemplo, se puede requerir asignar un ancho de banda limitado al tráfico P2P (BitTorrent) con el objetivo de no afectar al resto de tipos de tráfico de usuario. Considerando que existe un flujo definido por defecto para el tráfico de datos, la tarea consiste en definir un flujo adicional para el tráfico P2P, utilizando por ejemplo el puerto TCP de destino como criterio de coincidencia para OpenFlow.

- En el segundo paso, se crea el contenedor de tráfico (Create traffic container). Mediante el API correspondiente, se especifican, entre otros parámetros, el flujo de tráfico a ser asignado al contenedor y el ancho de banda dedicado. Posteriormente, la aplicación asigna valores a los atributos restantes del contenedor. El proceso es completado al agregar el contenedor creado en el servicio de Conmutación (Switching service), las redes de Entrega y Clasificación (Classification and Delivering networks) y la VNF de Clasificación \& Conformación (Classification \& Shaping VNF). Cada una de las entidades involucradas notifica a la aplicación sobre el resultado de la operación realizada.

- El tercer paso consiste en la definición y posterior creación de la SFC (Create SFC). Este proceso se inicia mostrando una lista de VNFs disponibles y sus capacidades respectivas para que el usuario seleccione las que formarán parte de la nueva SFC. Esta lista se obtiene al consultar el catálogo de VNFDs que la plataforma de NFV mantiene. Una vez que las VNFs han sido seleccionadas, la aplicación debe crear el descriptor de la SFC, denominado como VNFFGD. Este proceso involucra obtener determinados parámetros de especificación de las VNFs, así como seleccionar un contenedor de tráfico previamente definido y validado por el bloque funcional Traffic Container Handler para asociarlo a la SFC. La creación de la SFC implica que previamente se deben crear las instancias de las VNFs que la constituyen. Considerando que las instancias de VNF pasarán a formar parte de la instancia de vMANDO se debe realizar un proceso de actualización del NS. De acuerdo con el documento de Gestión y Orquestación de NFV propuesto por ETSI [42], la actualización del NS implica que el orquestador de NFV con base en los VNFDs proporcionados verifica la dependencia con otras instancias de VNF en ejecución, así como la disponibilidad de recursos para proceder posteriormente con la instanciación de las VNFs. Al término del proceso de instanciación, la aplicación incorpora el VNFFGD previamente creado al catálogo de NS para su validación. A continuación, se procede a la creación de la SFC dentro de vMANDO, indicando para ello el Id del descriptor previamente incorporado.

- Los pasos cuarto y quinto están relacionados al envío de notificaciones sobre el estado de contenedores de tráfico y SFCs (Traffic container push notification y SFC push notification). Las 
notificaciones son enviadas al cliente de la aplicación utilizando el método "push" ya que es una comunicación asíncrona iniciada por el back-end.

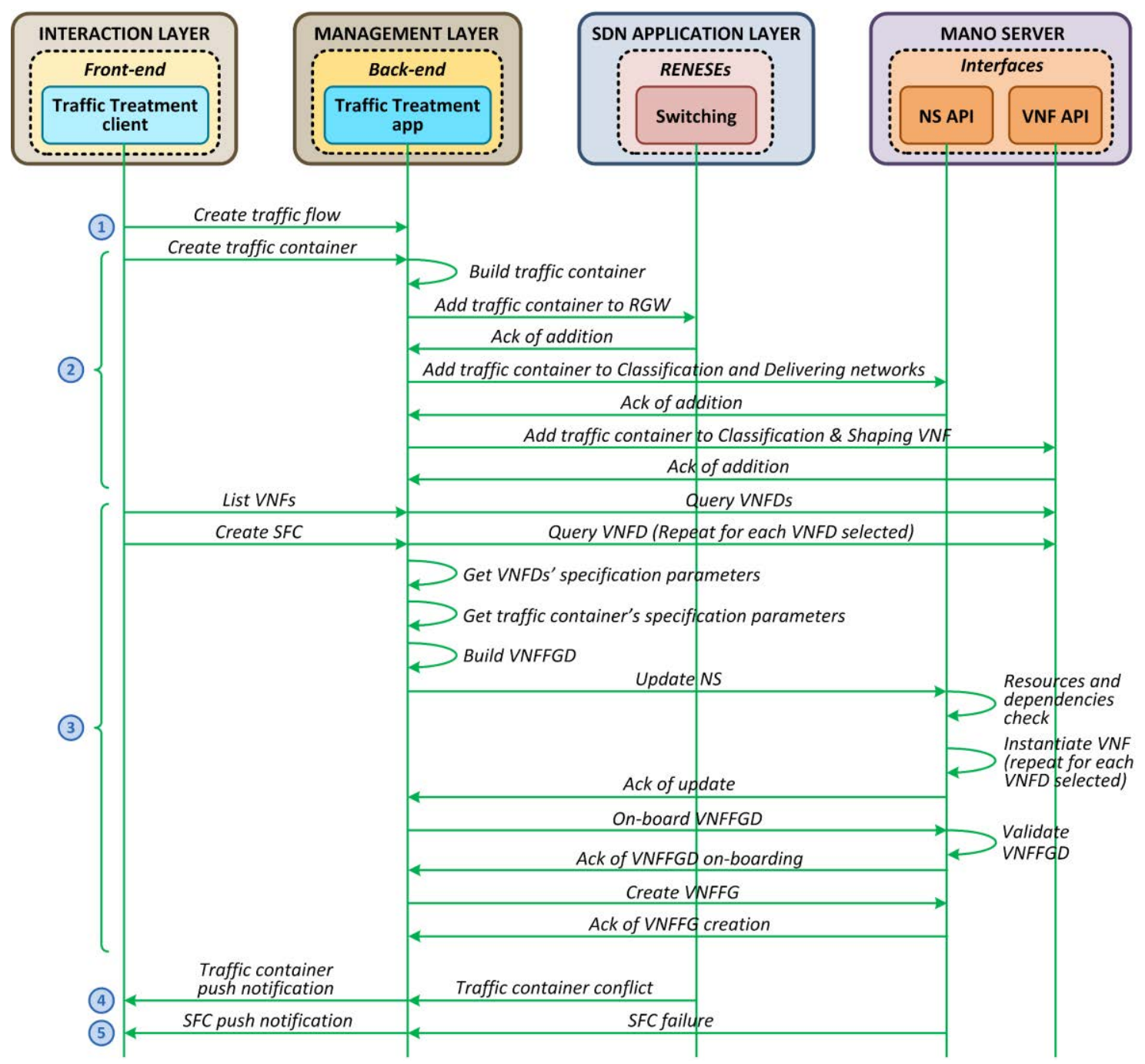

Figura 4.8 Proceso de implementación del tratamiento de tráfico diferenciado. La aplicación se comunica con el servicio de Conmutación mediante el protocolo RENESE. Por otra parte, la aplicación utiliza los servicios que proporciona NFV-MANO a través de las interfaces $N S$ y $V N F$. Con el fin de simplificar el gráfico, se ha omitido el cliente MANO de la Capa de Gestión.

Si bien la explicación que se proporciona está enfocada en el proceso de creación de un flujo de tráfico, contenedor y SFC, el resto de operaciones CRUD asociadas a los diferentes objetos siguen un patrón similar. Para utilizar una operación determinada del API es importante comprender su funcionalidad, considerar los parámetros de solicitud, así como interpretar los parámetros entregados en la respuesta. Esta información se muestra en detalle en las tablas complementarias que se presentan en el capítulo 5.

\subsection{Análisis de la Propuesta}

La propuesta de tratamiento de tráfico diferenciado permite que una red residencial basada en SDN y NFV proporcione mejores y nuevos servicios de conectividad de red los cuales cumplen satisfactoriamente con los requisitos de calidad de servicio (QoS) o procesamiento de tráfico que imponen las aplicaciones que se ejecutan en casa del usuario como el teletrabajo o el video bajo demanda. El tratamiento de tráfico diferenciado se fundamenta en el trabajo colaborativo y complementario de los componentes SDN y NFV el cual es coordinado por una aplicación RENEMA 
desde la arquitectura de gestión centrada en el usuario. El enfoque aprovecha el control preciso del tráfico que proporciona un RGW basado en SDN, la definición de las características de la red de acceso que permite una SDOAN y la capacidad implícita de NFV para crear SFCs específicas destinadas a procesar un determinado tipo de tráfico originado en la red residencial. Esta sección presenta un análisis de la propuesta considerando los siguientes temas de interés:

- Avance en el estado del arte. El análisis del estado del arte realizado en la sección 2.4.1 permitió concluir que existe un consenso en cuanto a la propuesta de virtualización del RGW basada en el paradigma NFV, siendo la arquitectura NERG de Broadband Forum y la iniciativa R-CORD los ejemplos más sobresalientes. Sin embargo, ambas propuestas presentan limitaciones. Por ejemplo, NERG no detalla el proceso a seguir para agregar más funciones al virtual Gateway (vG) y es agnóstico del paradigma SDN. Por otra parte, R-CORD solamente implementa los principios SDN en la red de acceso y su Gateway de Suscriptor virtual (vSG) no considera la creación de SFCs. Además, ambas propuestas no permiten que el usuario participe activamente de la gestión de su propia red. En este contexto, la propuesta de tratamiento de tráfico diferenciado con soporte de QoS basado en SDN y NFV conjuntamente con el enfoque centrado en el usuario representan una mejora significativa en comparación a NERG y R-CORD. Contribuyendo de esta manera, a avanzar en el estado del arte de las arquitecturas de gestión y despliegue de las redes residenciales.

- La Red Residencial como Servicio (RNaaS). La posibilidad de Gestionar y Controlar desde una ubicación centralizada todos los componentes funcionales de la red residencial como el RGW basado en SDN, la SDAN y las SFCs conjuntamente con la posibilidad de agrupar los componentes SDN y NFV en una única entidad como vMANDO, facilita en gran medida la automatización del despliegue de nuevas redes residenciales. Por otra parte, un enfoque basado en la Nube, como el que se muestra en la Figura 4.1, permite que el ISP integre los componentes de Gestión y Control y la mayoría de Recursos de Red y Computación en sus instalaciones (Oficina Central). De esta manera, el ISP estaría en la capacidad de proporcionar la Red Residencial como Servicio. En este escenario, se pretende que el ISP, a través de una plataforma web, oferte el Servicio de Red Residencial. En esta plataforma web el usuario de casa sería capaz de personalizar las características funcionales de la red residencial y del entorno de gestión con base en una configuración por defecto o básica que proporciona el ISP.

En lo que respecta a las características funcionales, el usuario determina las SFCs y, por ende, las VNFs que formaran parte del componente de Redes de vMANDO, así como especifica las características de la conexión a Internet a contratar como el ancho de banda ascendente y descendente. Por otra parte, en lo que respecta al entorno de gestión, el usuario determina las aplicaciones RENEMA que formarán parte del componente de Gestión de vMANDO. Existen aplicaciones orientadas al control del RGW basado en SDN y otras orientadas a la coordinación de los componentes SDN y NFV con el fin de proporcionar una funcionalidad específica como el tratamiento de tráfico diferenciado. Posterior a la puesta en marcha del servicio de red residencial, su gestión íntegra se la realiza desde el sistema de gestión centrado en el usuario.

- Requerimiento de una implementación completa y funcional de NFV-MANO. Ciertamente, la funcionalidad proporcionada por la aplicación de Tratamiento de Tráfico se soporta en las operaciones del ciclo de vida para descriptores e instancias que proporcionan las interfaces MANO definidas en el documento de Gestión y Orquestación de NFV propuesto por ETSI [42]. Por lo tanto, la implementación real de la propuesta requiere que la plataforma de NFV a utilizar implemente fielmente todas las funcionalidades y operaciones definidas por ETSI. En caso contrario, el tratamiento de tráfico diferenciado proporcionaría funcionalidades limitadas o no tendría un desempeño óptimo. Por ejemplo, para crear instancias de VNFFG es necesario previamente disponer de la respectiva plantilla de despliegue o descriptor en el cual se especifica la ruta que seguirá el tráfico a través de las distintas VNFs que componen vMANDO. Una vez que la instancia está en ejecución, la plataforma de NFV debería controlar las operaciones del ciclo de vida correspondientes en tiempo de ejecución, es decir, se debería permitir actualizar la ruta del tráfico sin necesidad de detener la instancia, actualizar el descriptor y crear una nueva instancia a partir del descriptor actualizado. 
- Interfaz Gráfica de Configuración para la VNF. Como se indicó en la sección 2.3.5, posterior a la instanciación de una VNF, se crea una interfaz de configuración que permite establecer los parámetros específicos de funcionamiento del servicio que proporciona la VNF. Según ETSI, esta interfaz es utilizada por el componente Gestión de Elementos (EM) o el Gestor de VNF (VNFM). Considerando el enfoque centrado en el usuario, esta interfaz de configuración también debería interactuar con el Sistema de Gestión de la red residencial ya que el usuario podría requerir modificar algún parámetro de la configuración de la VNF. De esta manera, al igual que el caso de las aplicaciones RENEMA, se podría proponer que cada VNF proporcione un cliente que se integraría a la Capa de Interacción de la Arquitectura de Gestión y que estaría encargado de interactuar con la interfaz de configuración de la VNF. Este cliente de VNF principalmente se encargaría de proporcionar una interfaz gráfica de configuración y realizaría las funciones del EM. Así, los principios $a$ y $b$ definidos en la sección 3.2.2, también serían válidos para los clientes de VNF.

- Sistemas inteligentes para soportar las tareas de gestión. Ciertamente, un enfoque centrado en el usuario permite simplificar significativamente las tareas de gestión de la red residencial. Por otra parte, en escenarios más avanzados, se podría implementar sistemas inteligentes que proporcionen soporte en las tareas de gestión. Por ejemplo, cada instancia de VNF tiene una configuración de inicio o por defecto la cual puede ser modificada durante el tiempo de vida de la instancia. Esta configuración de inicio puede no ser la adecuada considerando el estado actual del entorno virtual donde va a funcionar. En este sentido, se podría proponer un mecanismo que permita inferir una configuración inicial considerando diferentes parámetros funcionales del entorno de ejecución de la VNF como los recursos computacionales disponibles o la congestión de red.

De igual manera, se podría recomendar al usuario crear una determinada SFC con el objetivo de mejorar el rendimiento de la red residencial. Este Sistema de Recomendación de SFCs utilizaría información del estado de la red como las aplicaciones que se ejecutan en casa del usuario, el tipo de dispositivos utilizados, políticas de control parental o SFCs creadas previamente para proporcionar una recomendación específica.

\subsection{Conclusiones}

En el capítulo anterior, se destacó la necesidad de definir un modelo de trabajo colaborativo para los componentes SDN y NFV que forman parte de la arquitectura de gestión considerando que sus aportaciones individuales pueden resultar limitadas y, por lo tanto, pueden no cumplir con los requisitos de conectividad y procesamiento de tráfico que imponen determinadas aplicaciones que se ejecutan en la red residencial. Para abordar esta necesidad, en este capítulo se ha propuesto orquestar conjuntamente los componentes SDN y NFV para que la red residencial sea capaz de proporcionar un tratamiento específico con soporte de QoS al tráfico de las distintas aplicaciones que hacen uso de sus servicios y cumplir de esta manera con los requisitos impuestos. Con este enfoque se pretende enriquecer y ampliar los servicios de red que proporciona la red residencial al complementar las funcionalidades individuales que ofrecen los componentes SDN y NFV.

Para satisfacer las necesidades específicas de conectividad de una determinada aplicación, el tratamiento de tráfico diferenciado propone, en primer lugar, diferenciar el tráfico de una aplicación en la red residencial utilizando la capacidad del RGW basado en SDN para crear flujos de tráfico. En segundo lugar, se propone crear una SFC específica en vMANDO y asociarla al flujo de tráfico previamente creado en la red residencial. De esta manera, el componente NFV se encarga de crear la SFC que proporciona un tratamiento específico mientras que el componente SDN se encarga de diferenciar el tráfico de la aplicación y dirigirlo a la correspondiente SFC en vMANDO.

Ciertamente, este modo de trabajo colaborativo permite complementar las aportaciones individuales de SDN y NFV, no obstante, es necesario orquestar las operaciones a realizar tanto a nivel SDN como NFV. Es así, que se ha definido la aplicación de Tratamiento de Tráfico que interactúa con la Gestión y Orquestación (MANO) de NFV y el RGW basado en SDN para orquestar la implementación del tratamiento de tráfico diferenciado con base en los requerimientos del usuario. En este escenario, el 
usuario utiliza la aplicación para especificar, por una parte, la aplicación que tendrá un tratamiento específico y, por otra parte, en qué consistirá el tratamiento específico.

Si bien en el desarrollo del capítulo se ha puesto énfasis en la orquestación del RGW basado en SDN y NFV-MANO, también se ha evaluado la posible orquestación de la SDOAN desde el sistema de gestión centrado en el usuario lo cual permite obtener una flexibilidad multinivel que significativamente mejora las condiciones en las cuales la red residencial proporciona los servicios de conectividad. Este enfoque, al permitir controlar los recursos de red que proporciona el RGW basado en SDN, la SDOAN y las SFCs desde una ubicación centralizada, resulta de gran interés tanto para usuarios como para el ISP. El usuario puede personalizar su red con nuevos y mejores servicios destinados a proporcionar las condiciones necesarias para que las aplicaciones se desempeñen de forma adecuada y, como consecuencia, mejorar la Calidad de Experiencia (QoE) percibida. Por otra parte, el ISP puede proponer nuevos modelos de negocio para explotar los beneficios de agrupar los componentes SDN y NFV en vMANDO, siendo el ejemplo más representativo la provisión de la Red Residencial como Servicio (RNaaS). 


\section{Capítulo 5}

\section{Validación de las Propuestas}

\subsection{Introducción}

En los capítulos anteriores se realizó el diseño de la arquitectura de gestión y sus bloques funcionales. Se presentó un modelo de interacción que permitió comprender cómo se intercambia Información de Gestión entre las distintas capas que forman parte de la arquitectura y se explicó los estados de funcionamiento de aplicaciones RENENA y RENESEs. Adicionalmente, se diseñó una aplicación específica encargada de orquestar los componentes SDN y NFV que soportan la arquitectura de gestión propuesta para proporcionar un tratamiento de tráfico diferenciado con soporte de QoS a las aplicaciones que hacen uso de los servicios de conectividad que proporciona la red residencial.

En este capítulo, con el objetivo de validar las propuestas presentadas en esta Tesis Doctoral y analizar su factibilidad, en una primera parte, se presenta un caso de uso y su respectiva prueba de concepto. El caso de uso está enfocado en verificar el comportamiento global de la arquitectura y observar si ésta permite utilizar el enfoque centrado en el usuario y facilita su implementación. Para este fin, se han desarrollado dos aplicaciones RENEMA, denominadas como Control Parental y Estado de la Red, destinadas a mejorar los aspectos de la red residencial mencionados en la sección 2.1.4. Estas aplicaciones utilizan los servicios de Conmutación, Seguimiento de Dispositivos, NESA y NERON proporcionados por la capa de Aplicación SDN. En lo que respecta a la prueba de concepto, se implementaron todos los componentes arquitectónicos definidos para el caso de uso y se ha propuesto un escenario de validación compuesto por la Nube del ISP, diferentes redes residenciales y la red de acceso con el objetivo de implementar un prototipo real. No obstante, previo a la implementación del prototipo, se han creado bancos de prueba virtual con base en el escenario de validación para desarrollar la prueba de concepto, realizar experimentos y obtener resultados que han permitido verificar la factibilidad de las propuestas presentadas.

En una segunda parte, se aborda la implementación de la propuesta de tratamiento de tráfico diferenciado presentada en el capítulo 4, para lo cual se ha definido en detalle el conjunto de operaciones del API de la aplicación de Tratamiento de Tráfico. Adicionalmente, se explica el proceso a seguir para implementar vMANDO como un Servicio de Red (NS) y se proporcionan ejemplos de descriptores o plantillas de despliegue creadas con TOSCA. En la parte final se presenta un análisis sobre la idoneidad de Tacker y TOSCA para cumplir satisfactoriamente con los requisitos funcionales que plantea el tratamiento de tráfico diferenciado. 


\subsection{Caso de Uso}

\subsubsection{Definición de Componentes Arquitectónicos}

Como se mencionó en la sección 1.2, el objetivo principal de esta tesis doctoral consiste en proponer una nueva arquitectura que permita utilizar el enfoque centrado en el usuario para gestionar la red residencial a la vez que facilita su implementación. De esta manera, para verificar el cumplimiento de tal objetivo y validar las propuestas planteadas, se presenta un caso de uso compuesto por dos aplicaciones RENEMA a cargo de mejorar determinados aspectos de la red residencial como se explica a continuación:

- Control Parental (Parental Control). El objetivo de esta aplicación es mejorar la Facilidad de Gestión, Seguridad y $Q o S$. Considerando la gestión basada en políticas que propone el HGI [107], esta aplicación ayuda al usuario residencial a definir una política por dispositivo. Esta política se envía luego al servicio apropiado en la capa de Aplicación SDN el cual se encarga de instalar las entradas de flujo correspondientes en el RGW basado en SDN para hacer cumplir la política.

El usuario residencial también tiene la posibilidad de definir una política para dispositivos que se conectan a la red residencial por primera vez. Para este caso de uso, la política predeterminada o por defecto no proporciona ningún servicio de red hasta que el usuario residencial defina una política adecuada para el dispositivo que se conecta por primera vez.

La definición de políticas permite controlar la navegación en Internet. Para definir esta política se podrían considerar parámetros como la propiedad del dispositivo (Device), el horario de navegación (Schedule) y la acción de permitir o no la navegación (Action). La prioridad del servicio (Service priority) se ha agregado para demostrar y probar las capacidades de provisión de QoS de NERON. Además, se pueden agregar parámetros adicionales para colocar algún nombre (Name) a la política y alguna descripción (Description) que permitan expresar de mejor manera cual es el propósito de la política en las tareas de gestión. La Tabla 5.1 muestra la política definida para este caso de uso. Por otra parte, es importante mencionar que se pueden utilizar diferentes parámetros, como la dirección IP de destino o el identificador de VLAN, para definir políticas de navegación con base en requisitos de usuario adicionales o criterios de usabilidad distintos. En escenarios más avanzados, como las comunicaciones de emergencia, la aplicación debería permitir que dispositivos que se encuentran bloqueados para comunicaciones habituales se les permita la navegación mientras se realiza la comunicación de emergencia.

Tabla 5.1 Definición de políticas por medio de la aplicación de Control Parental.

\begin{tabular}{|l|l|}
\hline \multicolumn{1}{|c|}{ Parámetros } & \multicolumn{1}{c|}{ Valores } \\
\hline Device & Ricardo's tablet (MAC address) \\
\hline Schedule & $\begin{array}{l}\text { - Monday: } 18 \mathrm{~h} 00 \text { to } 20 \mathrm{~h} 00 \\
\text { - Saturday: } 15 \mathrm{~h} 00 \text { to } 18 \mathrm{~h} 00\end{array}$ \\
\hline Action & Block $\mid$ Allow \\
\hline Service priority & Low $\mid$ Medium | High \\
\hline Name & Some name (Optional) \\
\hline Description & Some description (Optional) \\
\hline
\end{tabular}

- Estado de la Red (Network Status). El objetivo de esta aplicación es mejorar la Visibilidad. La mejora de este aspecto implica mostrar información relevante para el usuario en un formato comprensible. Como se indicó en la sección 3.2.5, NESA y NERON se encargan de comunicar información sobre las estadísticas de red y el consumo de recursos de red, respectivamente. Así, la aplicación se encarga de recibir esta información con el objetivo de mostrar el estado actual de la red. Considerando que OpenFlow proporciona diferentes tipos de estadísticas relacionadas con puertos, flujos, colas, medidores, etc., las estadísticas de puerto se utilizan en este caso de uso para 
calcular el ancho de banda por puerto. El objetivo es proporcionar un historial de consumo de este recurso limitado con diferentes niveles de agregación (actual, día, semana y mes).

Con respecto al estado de los dispositivos de usuario, esta aplicación informa sobre su conexión o desconexión y el número de puerto asociado. Cuando un dispositivo está conectado a la red, su estado se muestra como "online", mientras que una desconexión se muestra como "offline".

Como se explicó en la sección 3.2.2, uno de los principios de diseño de la arquitectura plantea que se proporcionen diferentes Servicios de Red Residencial (RENESEs) para ocultar los detalles de configuración de red de bajo nivel y simplificar y agilizar el desarrollo de aplicaciones. De esta manera, para facilitar que se implemente el enfoque centrado en el usuario y que las aplicaciones proporcionen de forma satisfactoria las funcionalidades descritas previamente, se incluyen los siguientes RENESEs en la capa de Aplicación SDN:

- Conmutación (Switching). Considerando que uno de los servicios básicos que proporciona la red residencial es la conectividad, este servicio se encarga de permitir o no que un dispositivo pueda conectarse con otros dispositivos, lo cual representa la conectividad local, o conectarse con el Gateway para alcanzar Internet, lo cual representa la conectividad externa. Para este fin, este servicio permite agregar o eliminar un dispositivo del servicio de Conmutación. Para permitir la conectividad, la aplicación solicitante debe registrar la dirección MAC del dispositivo y la asignación de QoS proporcionada por NERON (par puerto-cola) en este servicio. Una vez que el servicio de Conmutación recibe los parámetros de configuración correctos, las entradas de flujo correspondientes se definen y se envían a la capa de control que se encarga de actualizar la configuración del RGW basado en SDN.

Ciertamente, se abstrae todo el proceso de conectividad de red básica a través de la provisión mejorada del servicio de Conmutación que incluye un nivel de seguridad adicional. De esta manera, la conectividad de red se proporciona o no con base en las instrucciones enviadas desde la capa superior, en este caso desde la aplicación de Control Parental.

- Seguimiento de Dispositivos (Device Tracking). Este servicio recibe Packet-Ins como eventos de red desde la Capa de Control para reconocer una conexión o desconexión de dispositivo y alertar a todas las aplicaciones y RENESEs suscritos mediante el envío de la notificación correspondiente. En el caso de uso, la aplicación de Control Parental utiliza la notificación de conexión de dispositivo para recuperar la política asociada, si ha sido previamente configurada, y determinar si se permite o no que el dispositivo sea provisto de conectividad local y externa. La aplicación de Estado de la Red también utiliza estas notificaciones para alertar al usuario sobre la conexión (online) o desconexión (offline) del dispositivo como se mencionó anteriormente. Finalmente, como se explicará posteriormente, NERON también utiliza estas notificaciones para realizar una distribución eficiente del ancho de banda considerando solamente dispositivos que están conectados y que tienen permiso de conectividad.

Es importante mencionar que, en el caso de uso planteado, el servicio de Seguimiento de Dispositivos solo notifica conexiones o desconexiones de dispositivos inalámbricos ya autenticados. Para dispositivos con cable, no se necesita autenticación o configuración IP previa.

- NESA. Con base en la definición realizada en la sección 3.2.5, este servicio implementa la función de Monitoreo la cual se encarga de sondear y recopilar periódicamente estadísticas de puerto del RGW basado en SDN. Principalmente, se muestrea el número de bytes recibidos y transmitidos para calcular el ancho de banda por puerto en bits por segundo (bps). Posteriormente, la función de Proveedor envía esta información periódicamente como una notificación de estadísticas a todas las aplicaciones y RENESEs suscritas a NESA.

- NERON. Como se explicó en la sección 3.2.5, este componente se encarga de admitir o no solicitudes de asignación de recursos en función del estado actual de los recursos de red el cual se obtiene del proceso de contabilidad que realiza. Con el fin de verificar el funcionamiento de NERON, se implementa una asignación de ancho de banda dinámica y se realiza una distribución equitativa del ancho de banda contratado de la conexión a Internet entre todos los puertos activos. Este mecanismo está destinado a distribuir de mejor manera el ancho de banda en la red residencial. 
Un puerto activo representa un puerto conectado a un dispositivo "online" el cual está configurado con una política de navegación que incluye la acción "allow". Con base en las notificaciones recibidas desde el servicio de Seguimiento de Dispositivos y las solicitudes recibidas desde la aplicación de Control Parental, se determina la cantidad actual de puertos activos. Cada puerto activo proporciona tres colas para ser asignadas. De esta manera, considerando el estado actual de las colas y la prioridad solicitada desde la aplicación de Control Parental, se realiza una asignación de recursos. Una explicación complementaria del algoritmo implementado para realizar la asignación de ancho de banda se presenta en la sección 5.3.4.

Con base en la información proporcionada por NESA, NERON también calcula el consumo de ancho de banda actual de los puertos activos, así como el ancho de banda disponible (no asignado). Esta información también se envía periódicamente como notificación de estado de recursos a todas las aplicaciones y RENESEs suscritos a NERON.

\subsubsection{Detalles Operacionales}

Esta sección muestra un ejemplo de la interacción entre todos los componentes arquitectónicos y permite complementar el funcionamiento de aplicaciones y servicios definido en la sección 3.4. Considerando que el proceso de Inicio ha sido realizado con éxito en todos los componentes y, tanto aplicaciones como servicios se encuentran en estado Activo, a continuación, se describen tres pasos básicos los cuales se muestran en la Figura 5.1:

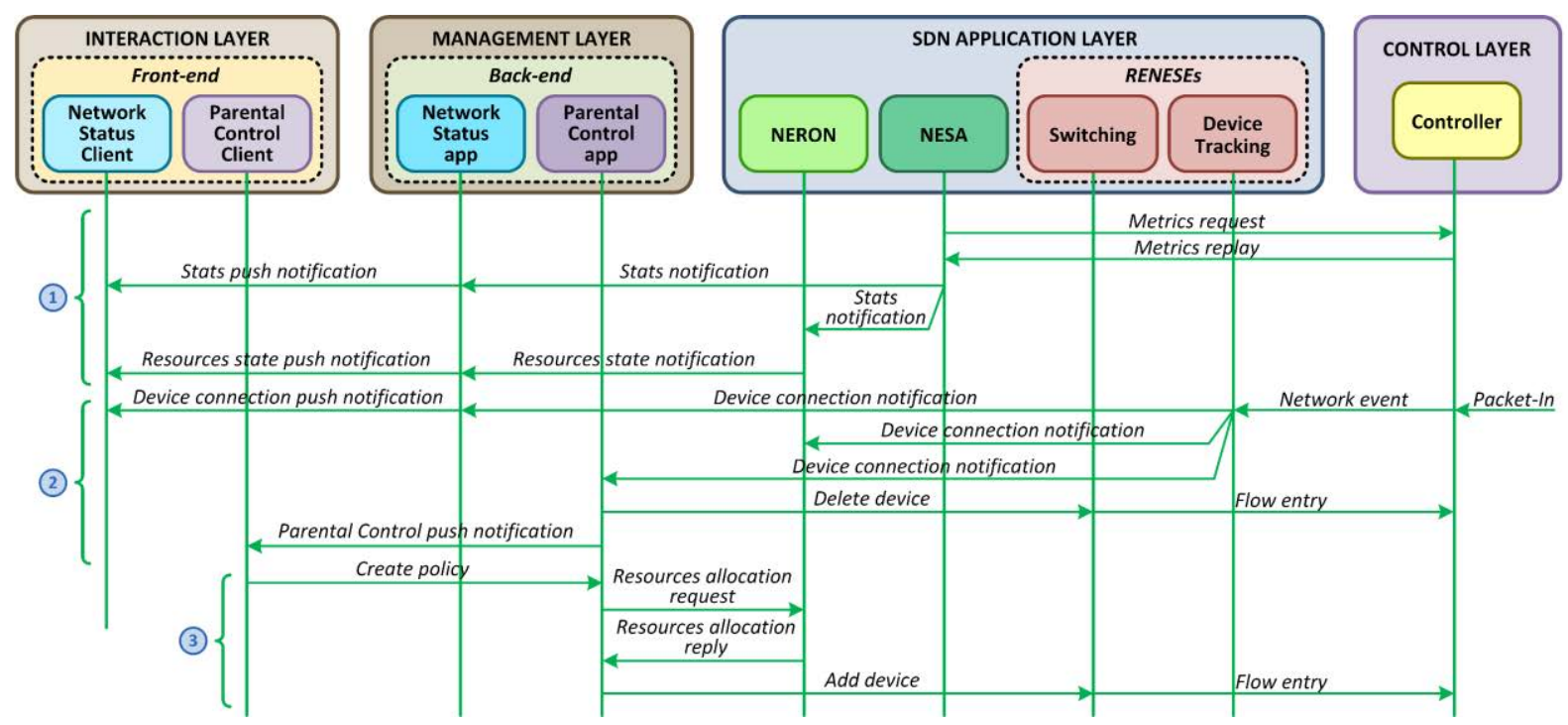

Figura 5.1 Interacción de los componentes arquitectónicos en el caso de uso. Nótese cómo los diferentes componentes back-end de las aplicaciones RENEMA interactúan con diferentes servicios a través del protocolo RENESE (intercambio de mensajes). Los componentes back-end y front-end interactúan entre sí a través del API REST. Con el objetivo de simplificar el gráfico, se han omitido ambos gestores.

- En primer lugar, se obtiene una vista inicial de la red. NESA recupera periódicamente métricas desde el RGW habilitado para OpenFlow y las envía a la aplicación de Estado de la Red y a NERON mediante una notificación de estadísticas (Stats notification). Con base en esta notificación, la aplicación de Estado de la Red crea una notificación formateada y la envía a su cliente correspondiente para mostrar las estadísticas (Stats push notificación). Por otra parte, NERON procesa la notificación para actualizar del estado de los recursos de red y enviar una notificación (Resources state notification) a la aplicación de Estado de la Red quien, posteriormente, crea y envía una notificación formateada a su respectivo cliente (Resources state push notification). Es importante mencionar, que las notificaciones enviadas son de tipo "push" considerando que son iniciadas por el servidor o back-end. La aplicación debería permitir configurar la periodicidad del envío de notificaciones push con el objetivo de no abrumar al usuario. Adicionalmente, se podría deshabilitar el envío de notificaciones push y solicitar ver las estadísticas bajo demanda utilizando el API REST. 
- En segundo lugar, como consecuencia de la conexión de un dispositivo a la red, la Capa de Control notifica tal conexión como un evento de red (Network event) al servicio de Seguimiento de Dispositivos. Este servicio procesa el evento de red y envía una notificación de conexión de dispositivo (Device connection notification) a tres entidades distintas. Con base en la notificación recibida, la aplicación de Estado de la Red crea y envía una notificación formateada a su cliente para informar sobre la nueva conexión (Device connection push notification) mientras que NERON actualiza el estado de la distribución de recursos de la red (ancho de banda disponible y puertos activos).

Por otra parte, la aplicación de Control Parental intenta recuperar una política desde el MIDB. Como el dispositivo se ha conectado por primera vez, la política no se ha definido previamente. De esta manera, la aplicación envía un mensaje RENESE al servicio de Conmutación con una acción de bloqueo (Delete device) para descartar todo el tráfico (política predeterminada). Además, la aplicación también envía una notificación al cliente para alertar al usuario sobre la acción realizada (Parental Control push notification).

- En tercer lugar, el cliente de la aplicación de Control Parental ayuda al usuario residencial a definir una política con base en los parámetros de la Tabla 5.1. La aplicación envía una solicitud de asignación de recursos (Resources allocation request) y, NERON, en función de la prioridad del servicio seleccionada, asigna los recursos disponibles especificando el par puerto-cola en la respuesta de asignación de recursos (Resources allocation reply). El par puerto-cola y la dirección MAC del dispositivo son utilizados por la aplicación de Control Parental para agregar el dispositivo al servicio de Conmutación (Add device). Finalmente, la entrada de flujo (Flow entry) correspondiente se crea y se envía a la Capa de Control para habilitar la conectividad local y externa del dispositivo.

Es importante mencionar que, en el caso de uso planteado, la aplicación es la encargada de realizar la solicitud de asignación de recursos para el dispositivo. No obstante, la solicitud de asignación de recursos podría estar implícita en el funcionamiento del servicio de Conmutación, es decir, el propio servicio se encargaría de solicitar recursos a NERON.

\subsection{Prueba de Concepto}

Se ha desarrollado una prueba de concepto (PoC) que incluye las aplicaciones RENEMA y RENESEs previamente definidos en el caso de uso planteado, así como el resto de bloques funcionales de la arquitectura de gestión definidos en la sección 3.2.2. Para la prueba de concepto también se ha propuesto un escenario de validación compuesto por la infraestructura de computación en la Nube del ISP (NFVI), las redes residenciales y la red de acceso.

\subsubsection{Detalles de Implementación}

En esta sección se proporcionan detalles de implementación de la arquitectura de gestión propuesta. En primer lugar, para implementar las capas de Control y de Aplicación SDN se ha utilizado el controlador Floodlight. Se ha utilizado este controlador considerando que las características funcionales de su arquitectura se ajustan a los propósitos demostrativos del caso de uso planteado. No obstante, la arquitectura de gestión propuesta en esta Tesis Doctoral es totalmente compatible con otros controladores SDN como OpenDaylight u ONOS presentados en la sección 2.2.4.

Por otra parte, considerando la descripción de funciones que deben ser realizadas por un determinado servicio de la capa de Aplicación SDN, algunos módulos de Floodlight se han modificado mientras que otros módulos se han desarrollado e integrado en la plataforma. Por ejemplo, el servicio de Conmutación está basado en el módulo "LearningSwitch" y NESA está basado en el módulo "Statistics". NERON y el servicio de Seguimiento de Dispositivos son módulos nuevos que han sido integrados en la plataforma. De esta manera, considerando este antecedente y los requisitos del caso de uso planteado, la Tabla 5.2 resume el conjunto de funciones o API proporcionado por NERON, NESA y los servicios de Conmutación (Switching) y Seguimiento de Dispositivos (Device Tracking). Como se explicó en la sección 3.2.5, las aplicaciones u otros servicios utilizan este conjunto de funciones a través del protocolo RENESE. Así, al momento de crear un mensaje se colocará como receptor (ReceiverId) el Id del 
RENESE y en el campo servicio (Service) se colocará la función específica a utilizar y sus respectivos argumentos (Arguments). La columna "API" en la Tabla 5.2 enumera todas las funciones que se proporcionan y que deben especificarse en el campo servicio del mensaje.

Como se aprecia en la tabla, la función de suscripción (subscription) es común entre todos los servicios. No obstante, NERON hace una distinción entre la suscripción a la función de asignación de recursos (Resources allocation subscription request) y la función de notificación del estado de recursos (Resources state subscription request), considerando que se puede requerir mostrar solamente el estado de los recursos sin la necesidad de solicitar una asignación en particular. A parte de las funciones definidas previamente, el servicio de Conmutación incluye dos funciones adicionales. La primera devuelve una lista con todos los dispositivos agregados al servicio (Added devices list request) lo cual permite saber que dispositivos actualmente disponen de conectividad local y externa. La segunda elimina todos los dispositivos del servicio (Clear added devices list) lo cual es útil cuando se necesita bloquear a todos los dispositivos con una sola acción.

Tabla 5.2 API basada en el intercambio de mensajes RENESE. En la capa de Aplicación SDN, NERON, NESA y los servicios de Conmutación y Seguimiento de Dispositivos proporcionan un conjunto de funciones o API para facilitar la gestión de la red residencial.

\begin{tabular}{|c|c|c|}
\hline RENESE & API & Argumentos \\
\hline \multirow{7}{*}{ NERON } & Resources allocation subscription request & - none \\
\hline & Resources allocation subscription reply & - successful or failed \\
\hline & Resources state subscription request & - none \\
\hline & Resources state subscription reply & - successful or failed \\
\hline & Resources allocation request & $\begin{array}{l}\text { - device_mac_address } \\
\text { - priority: high, medium or low } \\
\text { - port: } 1 \ldots . . . N \\
\text { - action: allocate or release }\end{array}$ \\
\hline & Resources allocation reply & $\begin{array}{l}\text { - device_mac_address } \\
\text { - feasibility: yes or } n o \\
\text { - port: } 1 \ldots . N \\
\text { - queue: } q 1, q 2 \text { or } q 3\end{array}$ \\
\hline & Resources state notification & $\begin{array}{l}\text { - available_bandwidth } \\
\text { - allocated_bandwidth_per_port } \\
\text { - allocated_bandwidth_per_queue } \\
\text { - active_ports } \\
\text { - total_bandwidth_consumption }\end{array}$ \\
\hline \multirow{3}{*}{ NESA } & Subscription request & - none \\
\hline & Subscription reply & - successful or failed \\
\hline & Stats notification & $\begin{array}{l}\text { - bandwidth_rx and bandwidth_tx per } \\
\text { port }\end{array}$ \\
\hline \multirow{7}{*}{ Switching } & Subscription request & - none \\
\hline & Subscription reply & - successful or failed \\
\hline & Add device & - device_mac_address, port, queue \\
\hline & Delete device & - device_mac_address, port, queue \\
\hline & Added devices list request & - none \\
\hline & Added devices list reply & - added_devices_list \\
\hline & Clear added devices list & - none \\
\hline \multirow{4}{*}{ Device Tracking } & Subscription request & - none \\
\hline & Subscription reply & - successful or failed \\
\hline & Device connection notification & - device_mac_address, port \\
\hline & Device disconnection notification & - device_mac_address, port \\
\hline
\end{tabular}


Como se explicó previamente, NESA recopila periódicamente estadísticas de puerto y posteriormente las envía como una notificación de estadísticas a sus suscriptores. Para el caso de uso, se ha establecido una frecuencia de 10 segundos. Esta frecuencia también se aplica a NERON considerando que cada vez que recibe una notificación de estadísticas realiza una actualización del estado de los recursos de red.

En segundo lugar, la Capa de Gestión ha sido implementada utilizando el lenguaje de programación Python v2.7 ${ }^{1}$. Para implementar el API REST se ha utilizado el micro framework Flask v0.10.1 ${ }^{2}$. Además, se ha utilizado PostgreSQL v9. $3^{3}$ para implementar la MIDB y Psycopg ${ }^{4}$ para implementar el correspondiente API. Como se explicó en la sección 3.2.4, cada aplicación RENEMA proporciona un conjunto de funciones de gestión a través de su API REST. De esta manera, la Tabla 5.3 resume el API REST de las aplicaciones de Control Parental y de Estado de la Red. En la tabla se detalla los URIs ${ }^{5}$ definidos, los métodos HTTP $^{6}$ utilizados y la descripción del API. Por otra parte, se ha utilizado la librería Requests ${ }^{7}$ de Python para emular el respectivo cliente de las aplicaciones implementadas y realizar pruebas de desempeño y de provisión de QoS.

Tabla 5.3 API de las aplicaciones RENEMA. En la Capa de Gestión, las aplicaciones de Control Parental y Estado de la Red exponen sus capacidades de gestión utilizando un API REST. En la capa Interacción, sus respectivos clientes usan esta API para permitir la gestión de la red residencial.

\begin{tabular}{|c|c|c|}
\hline URI & Método & API \\
\hline /renemaapps/parentalcontrol/policies & GET & List policies: lists policies defined \\
\hline /renemaapps/parentalcontrol/policies/<device $>$ & GET & $\begin{array}{l}\text { Show policy: shows information of a specific } \\
\text { policy }\end{array}$ \\
\hline /renemaapps/parentalcontrol/policies & POST & $\begin{array}{l}\text { Create policy: creates a policy by providing } \\
\text { the device's MAC address, the action, the } \\
\text { service priority, the schedule, a name and a } \\
\text { description }\end{array}$ \\
\hline$/$ renemaapps/parentalcontrol/policies $/<$ device $>$ & PUT & $\begin{array}{l}\text { Update policy: updates the values of certain } \\
\text { parameters of the policy }\end{array}$ \\
\hline /renemaapps/parentalcontrol/policies/ $<$ device $>$ & DELETE & Delete policy: deletes a specific policy \\
\hline /renemaapps/parentalcontrol/policies/clear & GET & $\begin{array}{l}\text { Clear policies: deletes all policies stored in } \\
\text { MIDB }\end{array}$ \\
\hline /renemaapps/networkstatus/stats & GET & $\begin{array}{l}\text { List stats: lists the bandwidth consumption of } \\
\text { all devices }\end{array}$ \\
\hline /renemaapps/networkstatus/stats/ $<$ device $>$ & GET & $\begin{array}{l}\text { Show stat: shows the detailed bandwidth } \\
\text { consumption of a specific device }\end{array}$ \\
\hline /renemaapps/networkstatus/resources & GET & $\begin{array}{l}\text { Show network resources: shows the global } \\
\text { state of network resources }\end{array}$ \\
\hline$/$ renemaapps/networkstatus/resources $/<$ device $>$ & GET & $\begin{array}{l}\text { Show device resources: shows the state of } \\
\text { network resources allocated to a specific } \\
\text { device }\end{array}$ \\
\hline /renemaapps/networkstatus/devices & GET & $\begin{array}{l}\text { List devices: lists online devices and offline } \\
\text { devices }\end{array}$ \\
\hline$/$ renemaapps/networkstatus/devices/ $<$ device $>$ & GET & $\begin{array}{l}\text { Show device: shows the state of a specific } \\
\text { device }\end{array}$ \\
\hline
\end{tabular}

\footnotetext{
${ }^{1}$ https://www.python.org/

${ }^{2} \mathrm{http}: / /$ flask.pocoo.org/

${ }^{3} \mathrm{https}: / /$ www.postgresql.org/

${ }^{4}$ http://initd.org/psycopg/

${ }^{5}$ URI (Uniform Resource Identifier) es una cadena de caracteres que identifica los recursos de una red de forma unívoca.

${ }^{6}$ HTTP (Hypertext Transfer Protocol) permite realizar una petición de datos y recursos. Es la base de intercambio de datos en la Web.

${ }^{7} \mathrm{http}$ //docs.python-requests.org/en/master/
} 
Con respecto a la aplicación de Control Parental, el API proporciona un conjunto de operaciones CRUD para las políticas que controlan la navegación en Internet. Por otra parte, la aplicación de Estado de la Red principalmente recupera información de estado desde la MIDB para mantener informado al usuario. La Tabla 5.4 y la Tabla 5.5 muestran en detalle los parámetros de Solicitud y Respuesta del API REST de la aplicación de Control Parental y de Estado de la Red respectivamente. Al igual que en el caso de la aplicación de Tratamiento de Tráfico presentada en la sección 4.4.4, existen parámetros o atributos que son asignados por el usuario al momento de realizar la solicitud, como por ejemplo la prioridad del servicio (service priority) en una política, mientras que otros son asignados por la propia aplicación, como por ejemplo la fecha de creación (creation_date).

Tabla 5.4 Parámetros de Solicitud y Respuesta del API REST de la aplicación de Control Parental.

\begin{tabular}{|c|c|c|c|c|}
\hline \multirow{2}{*}{ API } & \multicolumn{2}{|c|}{ Parámetros de Solicitud } & \multicolumn{2}{|c|}{ Parámetros de Respuesta } \\
\hline & Nombre & Tipo & Nombre & Tipo \\
\hline List policies & none & none & defined_policies & array \\
\hline \multirow{8}{*}{ Show policy } & \multirow{8}{*}{ device_mac_address } & \multirow{8}{*}{ string } & device_mac_address & string \\
\hline & & & schedule & array \\
\hline & & & action & string \\
\hline & & & service_priority & integer \\
\hline & & & name & string \\
\hline & & & description & string \\
\hline & & & creation_date & string \\
\hline & & & update_date & string \\
\hline \multirow{8}{*}{ Create policy } & device_mac_address & string & device_mac_address & string \\
\hline & schedule & array & schedule & array \\
\hline & action & string & action & string \\
\hline & service_priority & integer & service_priority & integer \\
\hline & \multirow{2}{*}{ name } & \multirow{2}{*}{ string } & name & string \\
\hline & & & description & string \\
\hline & \multirow{2}{*}{ description } & \multirow{2}{*}{ string } & creation_date & string \\
\hline & & & update_date & string \\
\hline \multirow{8}{*}{ Update policy } & device_mac_address & string & device_mac_address & string \\
\hline & schedule & array & schedule & array \\
\hline & action & string & action & string \\
\hline & service_priority & integer & service_priority & integer \\
\hline & \multirow{2}{*}{ name } & \multirow{2}{*}{ string } & name & string \\
\hline & & & description & string \\
\hline & \multirow{2}{*}{ description } & \multirow{2}{*}{ string } & creation_date & string \\
\hline & & & update_date & string \\
\hline Delete policy & device_mac_address & string & result & string \\
\hline Clear policies & none & none & result & string \\
\hline
\end{tabular}

En lo que se refiere a las políticas de navegación en Internet, no existe ninguna restricción para eliminar una política que actualmente está en uso. Si este fuese el caso, el servicio de Conmutación al recibir una orden de eliminar el dispositivo (Delete device) removería la entrada de flujo que permite la conectividad para posteriormente instalar una entrada de flujo para descartar (Drop) todo el tráfico del dispositivo en cuestión. Para completar la descripción de los componentes de la capa de Gestión y de Aplicación SDN, la Tabla 5.6 resume el conjunto de funciones o API que proporcionan los Gestores correspondientes. Considerando los requerimientos de la prueba de concepto, principalmente se han implementado las 
funciones que permiten coordinar el proceso de comunicación entre las capas de Gestión y Aplicación SDN, así como la asignación de un identificador de capa. En el caso del Gestor de RENESE también se incluye la función de descubrimiento de servicio.

Tabla 5.5 Parámetros de Solicitud y Respuesta del API REST de la aplicación de Estado de la Red.

\begin{tabular}{|c|c|c|c|c|}
\hline \multirow{2}{*}{ API } & \multicolumn{2}{|c|}{ Parámetros de Solicitud } & \multicolumn{2}{|c|}{ Parámetros de Respuesta } \\
\hline & Nombre & Tipo & Nombre & Tipo \\
\hline \multirow{2}{*}{ List stats } & \multirow{2}{*}{ none } & \multirow{2}{*}{ none } & device_mac_address & \multirow{2}{*}{ array } \\
\hline & & & bw_consumption & \\
\hline \multirow{5}{*}{ Show stat } & \multirow{5}{*}{ device_mac_address } & \multirow{5}{*}{ string } & device_mac_address & string \\
\hline & & & device_port & integer \\
\hline & & & port_bandwidth_tx & float \\
\hline & & & port_bandwidth_rx & float \\
\hline & & & timestamp & string \\
\hline \multirow{5}{*}{$\begin{array}{l}\text { Show network } \\
\text { resources }\end{array}$} & \multirow{5}{*}{ none } & \multirow{5}{*}{ none } & active_ports & integer \\
\hline & & & bandwidth_consumption & float \\
\hline & & & available_bandwidth & float \\
\hline & & & hired_bandwidth & float \\
\hline & & & timestamp & string \\
\hline \multirow{5}{*}{$\begin{array}{l}\text { Show device } \\
\text { resources }\end{array}$} & \multirow{5}{*}{ device_mac_address } & \multirow{5}{*}{ string } & device_mac_address & string \\
\hline & & & queues_bandwidth: q1, q2, q3 & float \\
\hline & & & device_port & integer \\
\hline & & & port_bandwidth & float \\
\hline & & & timestamp & string \\
\hline \multirow{2}{*}{ List devices } & \multirow{2}{*}{ none } & \multirow{2}{*}{ none } & online_devices & array \\
\hline & & & offline_devices & array \\
\hline \multirow{4}{*}{ Show device } & \multirow{4}{*}{ device_mac_address } & \multirow{4}{*}{ string } & device_mac_address & string \\
\hline & & & policy_state & string \\
\hline & & & device_port & string \\
\hline & & & device_state & string \\
\hline
\end{tabular}

Tabla 5.6 Servicios proporcionados por el Gestor de aplicación RENEMA y el Gestor de RENESE. Cada tiene su propio prefijo para identificar aplicaciones y servicios.

\begin{tabular}{|l|l|l|}
\hline \multicolumn{1}{|c|}{ Entidad } & \multicolumn{1}{c|}{ API } & \multicolumn{1}{c|}{ Argumentos } \\
\hline \multirow{4}{*}{$\begin{array}{l}\text { RENEMA app } \\
\text { Manager }\end{array}$} & RENEMA app id Allocation Request & - none \\
\cline { 2 - 3 } & RENEMA app id Allocation Reply & - renema_app_id (ml-app-xxx) \\
\cline { 2 - 3 } & RENESE Message Reception & - renese_message (TCP socket) \\
\cline { 2 - 3 } & RENESE Message Sending & - renese_message (TCP socket) \\
\hline \multirow{5}{*}{$\begin{array}{l}\text { Manager } \\
\text { RENESE }\end{array}$} & RENE id Allocation Request & - none \\
\cline { 2 - 3 } & RENESE id Allocation Reply & - renese_id (sdnal-ns-yyy) \\
\cline { 2 - 3 } & RENESE Registration & - renese_id, service_description \\
\cline { 2 - 3 } & RENESE Discovery Request & - required_service \\
\cline { 2 - 3 } & RENESE Discovery Reply & - renese_id (sdnal-ns-yyy) \\
\cline { 2 - 3 } & RENESE Message Reception & - renese_message (TCP socket) \\
\cline { 2 - 3 } & RENESE Message Sending & - renese_message (TCP socket) \\
\cline { 2 - 3 } & RENESE Message Forwarding & - renese_message (between two reneses) \\
\hline
\end{tabular}


Finalmente, se han elaborado diagramas de estado para los diferentes componentes de la arquitectura que intervienen en la prueba de concepto con base en la información de funcionamiento de aplicaciones RENEMA y RENESEs proporcionada en las secciones 3.4.3 y 3.4.4 respectivamente. Así, por una parte, la Figura 5.2 y la Figura 5.3 muestran los diferentes estados de la aplicación de Control Parental en el procesamiento de solicitudes realizadas al API REST y de mensajes RENESE respectivamente.

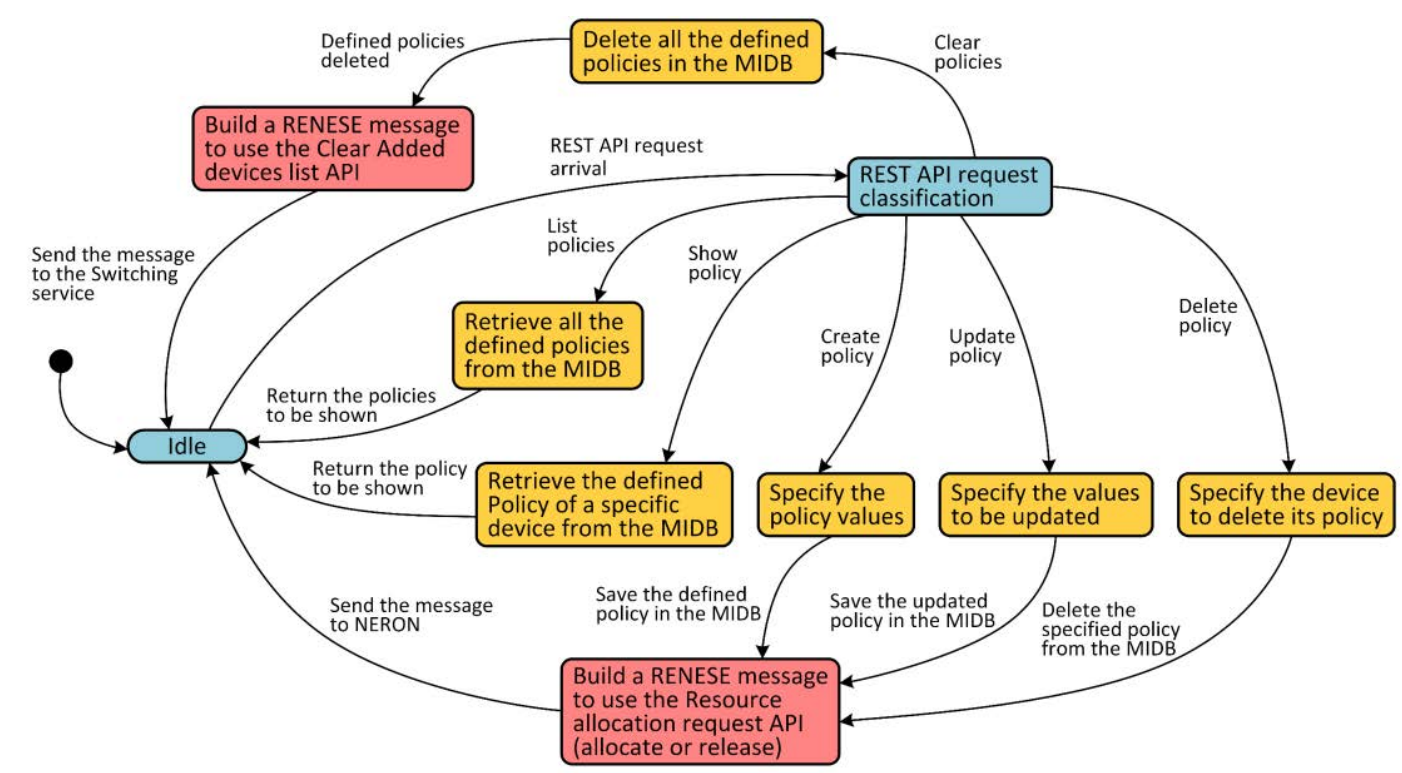

Figura 5.2 Diagrama de estado de la aplicación de Control Parental para procesar solicitudes realizadas al API REST.

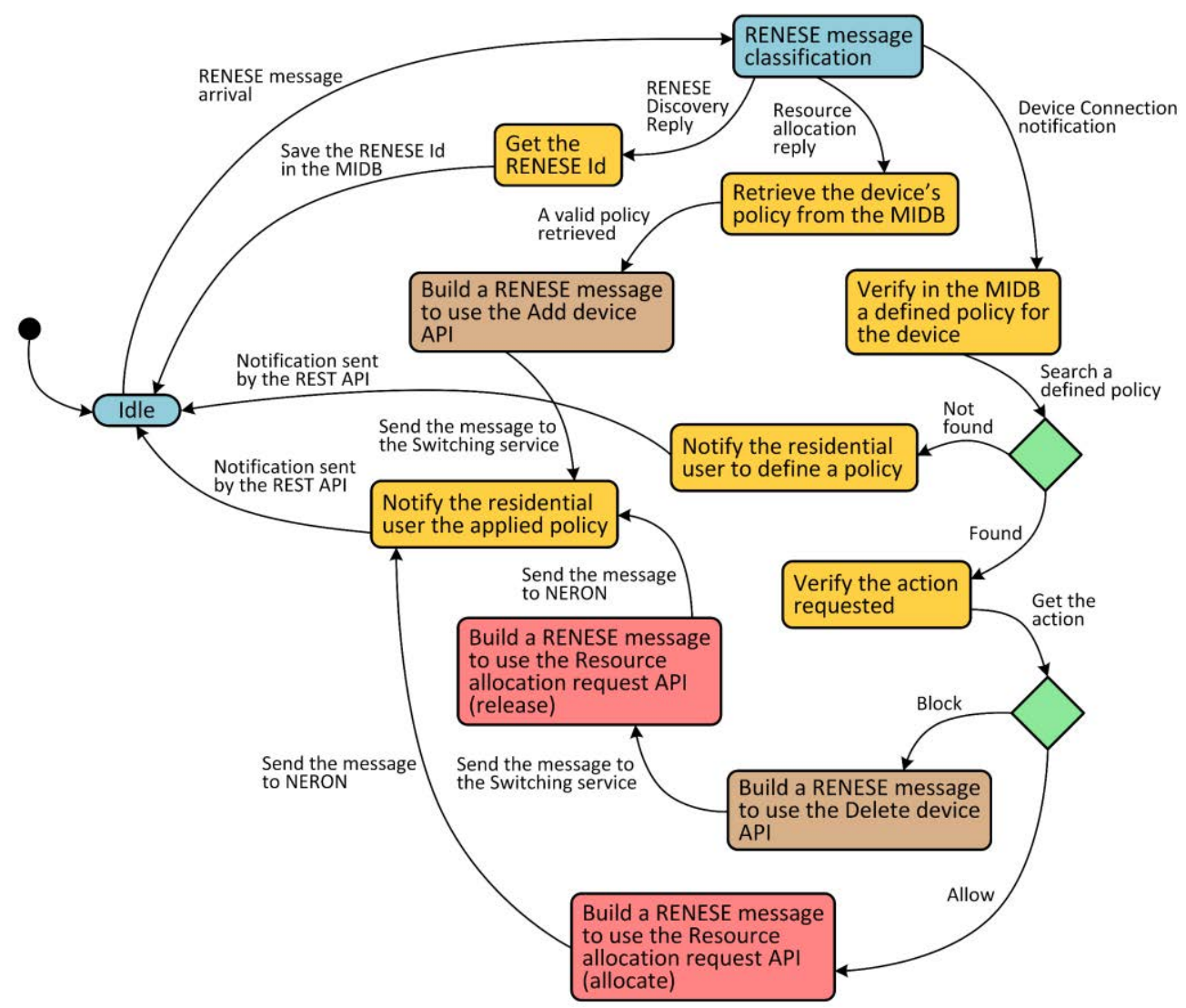

Figura 5.3 Diagrama de estado de la aplicación de Control Parental para procesar mensajes RENESE. 
Por otra parte, la Figura 5.4 y la Figura 5.5 muestran los diferentes estados del servicio de Conmutación en el procesamiento de Packet-Ins y de mensajes RENESE respectivamente. En el Apéndice A se incluye el resto de diagramas de estado y se invita al lector a consultarlo si desea comprender de mejor manera cómo se implementaron los diferentes componentes arquitectónicos de la prueba de concepto.

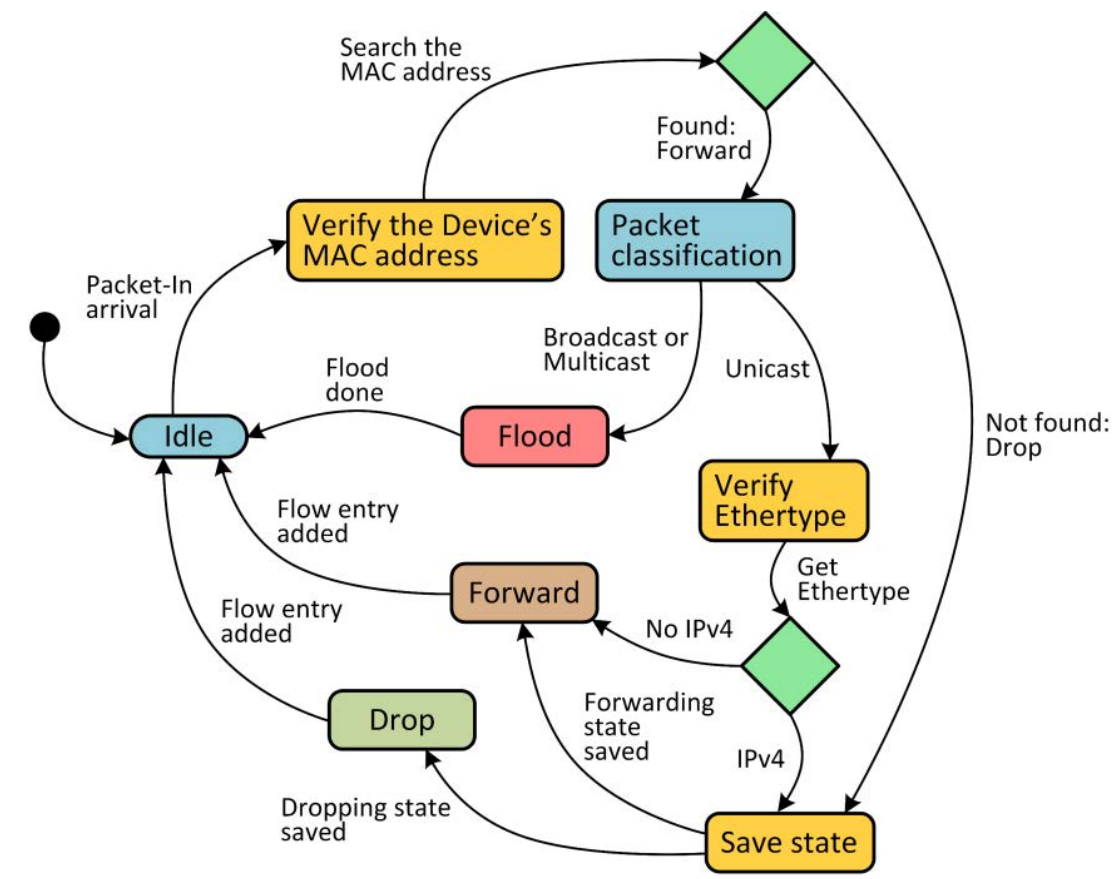

Figura 5.4 Diagrama de estado del servicio de Conmutación para procesar Packet-Ins.

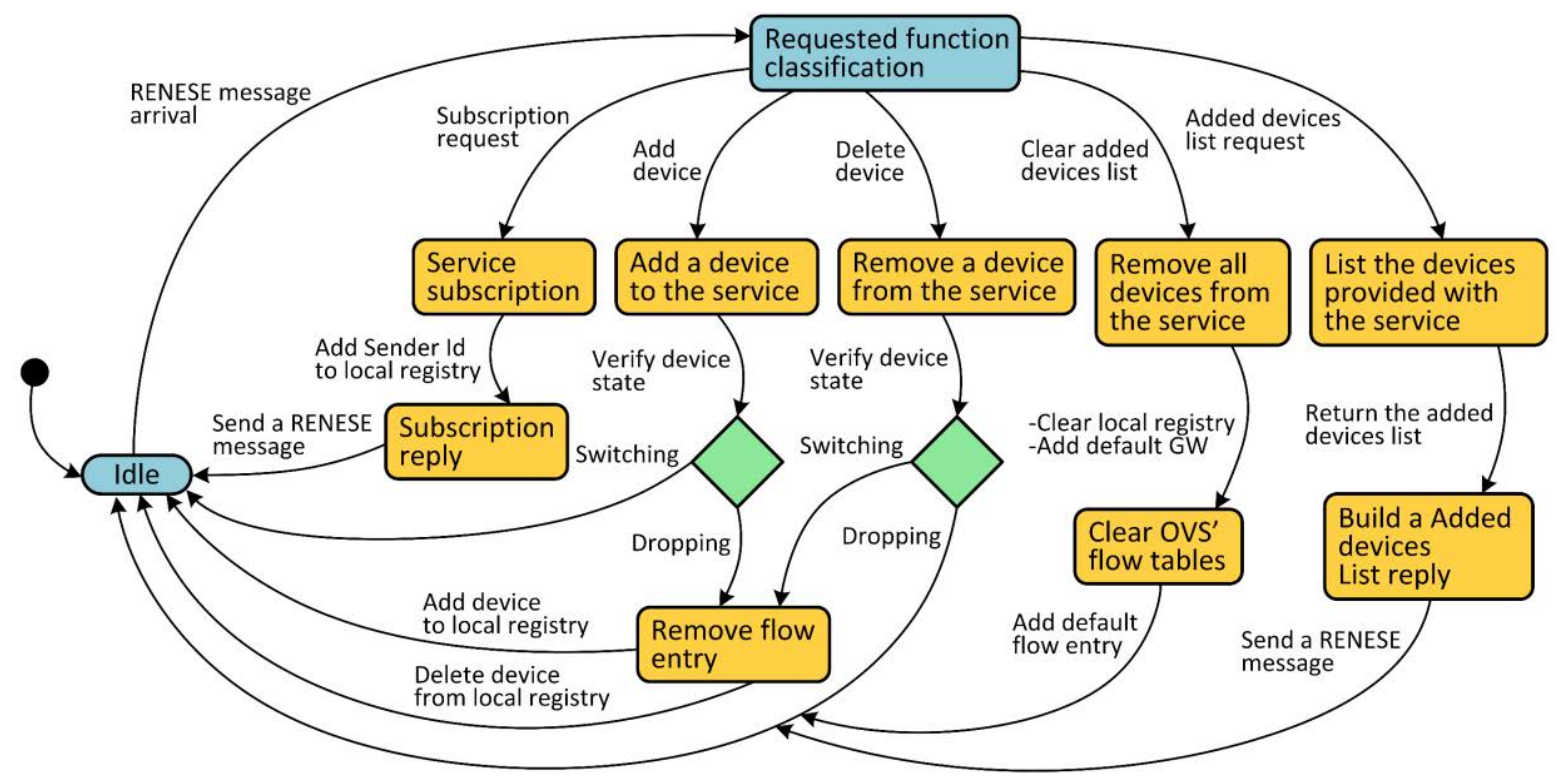

Figura 5.5 Diagrama de estado del servicio de Conmutación para procesar mensajes RENESE. 


\subsubsection{Escenario de Validación}

El objetivo final de las propuestas presentadas en esta tesis doctoral, es proporcionar una configuración de red como la que se muestra en la Figura 4.1. De esta manera, el usuario mediante el uso de diferentes aplicaciones RENEMA destinadas a controlar el RGW basado en SDN, la red de acceso y el conjunto de SFCs que se despliegan en la NFVI, puede personalizar con un alto grado de flexibilidad los servicios de conectividad y complementarios que sustentará la red residencial. En este contexto, y con el objetivo de desarrollar la prueba de concepto, se propone un escenario de validación como el que se muestra en la Figura 5.6. Para este fin, se pretende desplegar una red de acceso óptica real basada en el estándar GPON (Gigabit-capable Passive Optical Network) utilizando equipo del proveedor TELNET-RI ${ }^{8}$, en particular la OLT Smart OLT240 y la ONT WaveAccess 511. Además, se pretende que el sistema de control de la GPON, denominado como TGMS (TELNET GPON Management System), sea compatible con los principios de SDN y proporcione un API que permita configurar las características de la red de acceso como se explicó en la sección 4.2.

En las instalaciones del usuario, conjuntamente con la ONT, se coloca un Banana $\mathrm{Pi}^{9}$ (Bpi) que cumplirá con las funciones de RGW basado en SDN. La Banana Pi es una placa de desarrollo basada en router que incluye 4 puertos $\mathrm{Gb}$ Ethernet que actúan como puertos LAN, un puerto Gb Ethernet que actúa como puerto WAN y una interfaz WLAN. Considerando que la Bpi no soporta de forma nativa OpenFlow, se instalará un Open vSwitch (OVS) y sus puertos se los asociará a los puertos físicos LAN y WLAN. En un futuro cercano se espera que tanto la OLT y la ONT soporten de forma nativa OpenFlow o cualquier protocolo estándar de la interfaz Southbound de la arquitectura SDN.

Con base en el enfoque de gestión basado en la Nube, en la central de telecomunicaciones del ISP se coloca una infraestructura de computación en la Nube utilizando OpenStack conjuntamente con la OLT. Desde la perspectiva del paradigma NFV, considerando que OpenStack ya proporciona el NFVI y el correspondiente Gestor de Infraestructura Virtualizada (VIM), los componentes restantes de MANO como el Orquestador de NFV (NFVO) y el Gestor de VNF (VNFM) deberían agregarse para permitir el despliegue de los diferentes componentes de vMANDO (Instancia de Gestión y SFCs). En este sentido, se podría utilizar cualquiera de las plataformas de NFV que se presentaron en la sección 2.3.6. No obstante, para fines demostrativos se ha seleccionado Tacker como NFVO y VNFM que implementa las interfaces NFV-MANO requeridas para desplegar y operar vMANDOs.

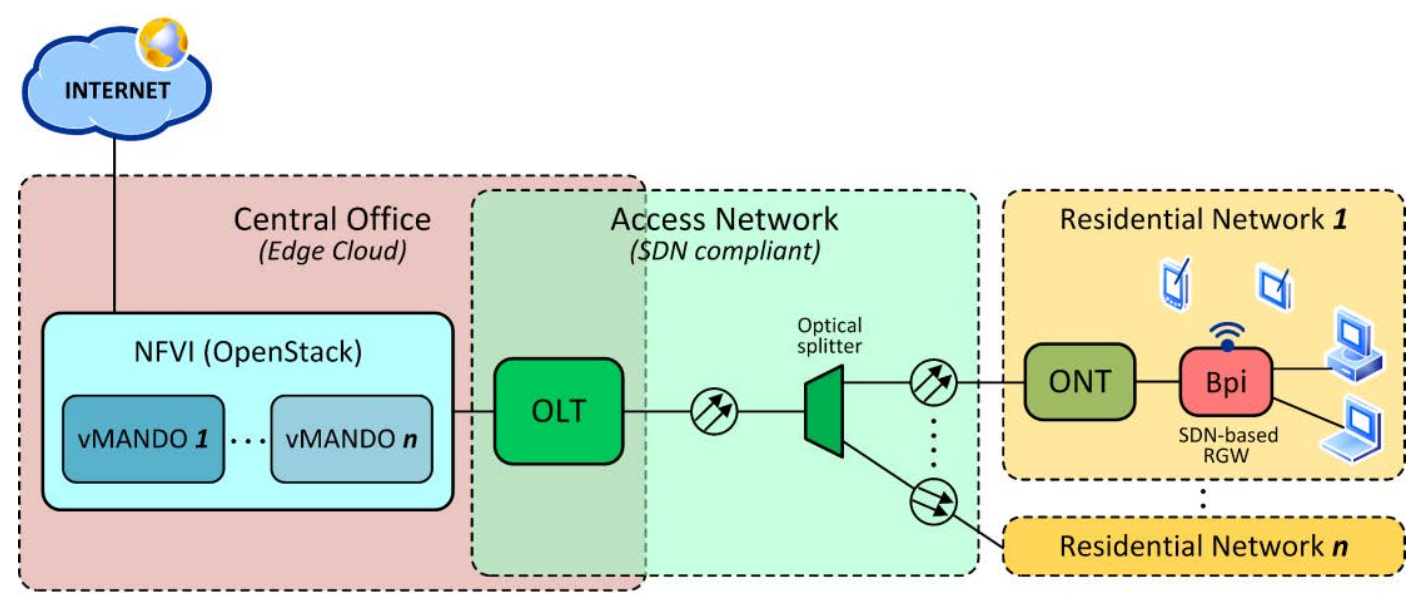

Figura 5.6 Escenario de validación propuesto. Nótese como se integran la red residencial, la red de acceso y la NFVI ubicada en la central de telecomunicaciones del ISP (Central Office). Para simplificar el gráfico no se ha incluido el componente de gestión y control.

Es importante mencionar que el escenario de validación descrito forma parte del proyecto GREDOS el cual será descrito en el siguiente capítulo. Al tiempo que se escribe esta memoria, el escenario de la

\footnotetext{
${ }^{8} \mathrm{http}: / /$ www.telnet-ri.es/en

${ }^{9} \mathrm{http}: / /$ www.banana-pi.org/r2.html
} 
Figura 5.6 está siendo implementado en el proyecto con el objetivo de proporcionar un prototipo real con base en los resultados obtenidos de esta tesis doctoral. Considerando este hecho, en una etapa anterior, el escenario de validación propuesto fue implementado como un banco de pruebas virtual para desarrollar la prueba de concepto y realizar pruebas de desempeño y de provisión de QoS que permitieron verificar la factibilidad de las propuestas presentadas y obtener tales resultados.

La primera versión del banco de pruebas virtual se implementó con máquinas virtuales LXC orquestadas con la herramienta Virtual Networks over Linux (VNX) [108] como se muestra en la Figura 5.7. El banco de pruebas virtual se ejecutó en un sistema Linux Ubuntu 14.04 equipado con un procesador Intel i7-3770 (3,40 GHz) y 8 GB de RAM. Cada VM en el escenario emuló un vMANDO, un vCSD o un dispositivo de usuario. Para proporcionar un escenario realista, se incluyeron tres redes residenciales. Por consiguiente, tres máquinas virtuales representaron los vMANDOs a cargo de alojar los componentes SDN y NFV de cada red residencial. Otra máquina virtual representó el vCSD para alojar los servicios DHCP y DNS. Las VNFs se implementaron utilizando el Forwarding IP de Linux para el encaminamiento e IPTables de Linux para el NAT. Además, al utilizar un control en banda se definieron dos LAN virtuales (VLAN) por red residencial para aislar el tráfico de gestión del tráfico de datos.

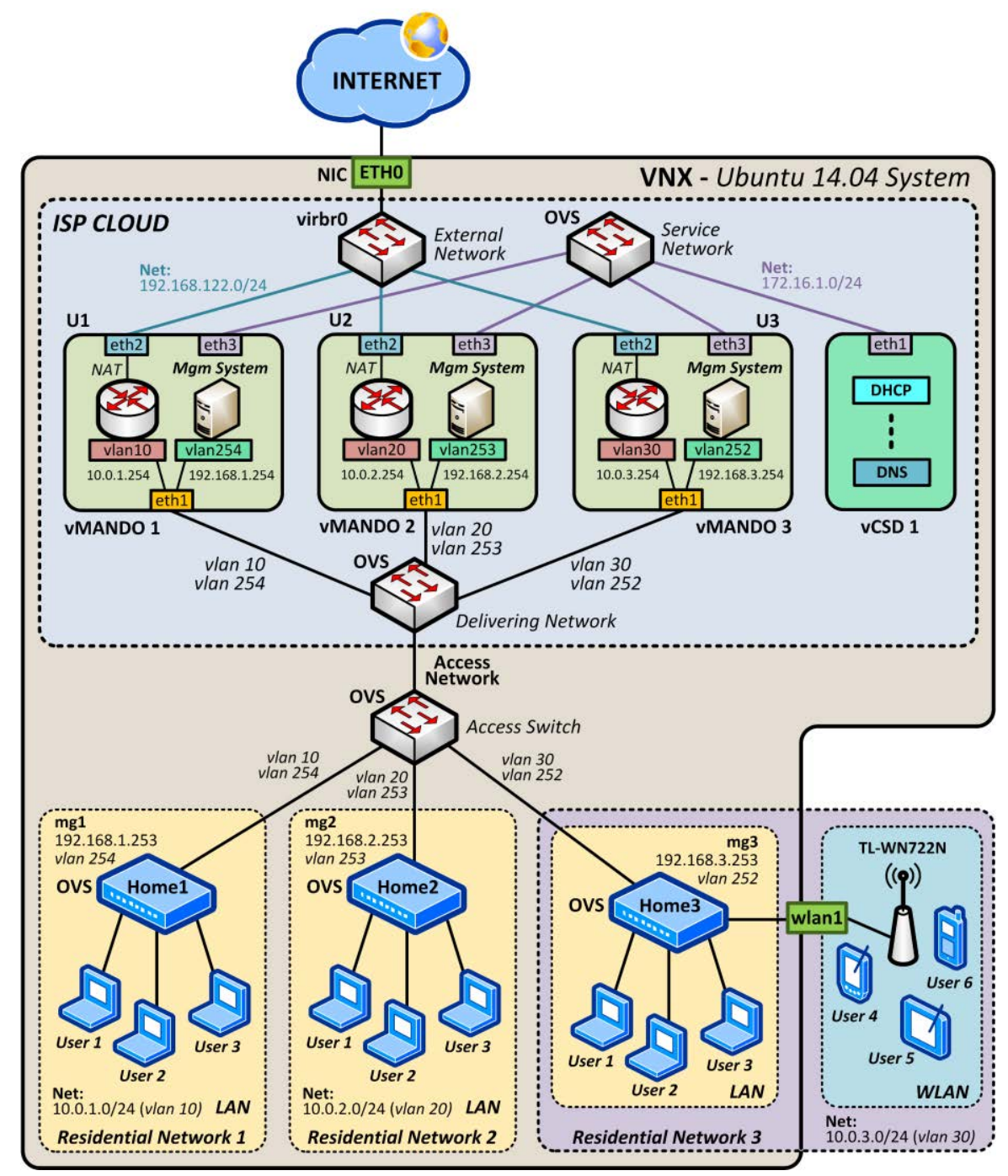

Figura 5.7 Primera versión del banco de pruebas virtual desarrollado para la prueba de concepto. Para emular la Nube del ISP se utiliza principalmente máquinas virtuales.

En el lado de la red residencial, se utilizó Open vSwitch v2.4.0 (OVS) como RGW basado en SDN, designados como Home1, Home2 y Home3 en la Figura 5.7. Además, cada OVS contó con una interfaz de gestión (designada como mg1 para Home1) que se utilizó para establecer la conexión de control en 
banda con el plano de control correspondiente (designado como VLAN 254 para Home1). La cantidad de dispositivos de usuario conectados es variable y depende del tipo de prueba que se realizará. Además, se agregó un adaptador USB inalámbrico (TL-WN722N) que actúa como punto de acceso (AP) utilizando el daemon hostapd ${ }^{10}$. De esta manera, se proporciona acceso a la WLAN del escenario de experimentación a dispositivos externos como laptops, tablets o smartphones.

En la segunda versión del banco de pruebas virtual, se utilizó OpenStack para implementar la Nube del ISP. De igual manera se utilizó la herramienta VNX en un sistema Linux Ubuntu 14.04.4 equipado con un procesador Intel Xeon-E5440 (2,83 GHz) y $17 \mathrm{~GB}$ de RAM. El banco de pruebas virtual se compone de dos partes: escenarios de VNX $A$ y $B$ como se muestra en la Figura 5.8. Con base en el tutorial de OpenStack Liberty ${ }^{11}$, el escenario de VNX $A$ representa la infraestructura en la Nube del ISP la cual se compone de dos máquinas virtuales LXC (nodos de Controlador y Red) y dos máquinas virtuales KVM (nodos de Cómputo). Basándose en una configuración estándar, estas máquinas virtuales están interconectadas a través de la infraestructura de red en la Nube la cual se compone de cuatro redes: ExtNet para la conectividad a Internet, DeliveringNet para conexiones basadas en VLAN, TunnNet para conexiones basadas en túneles y MgmtNet para la gestión.

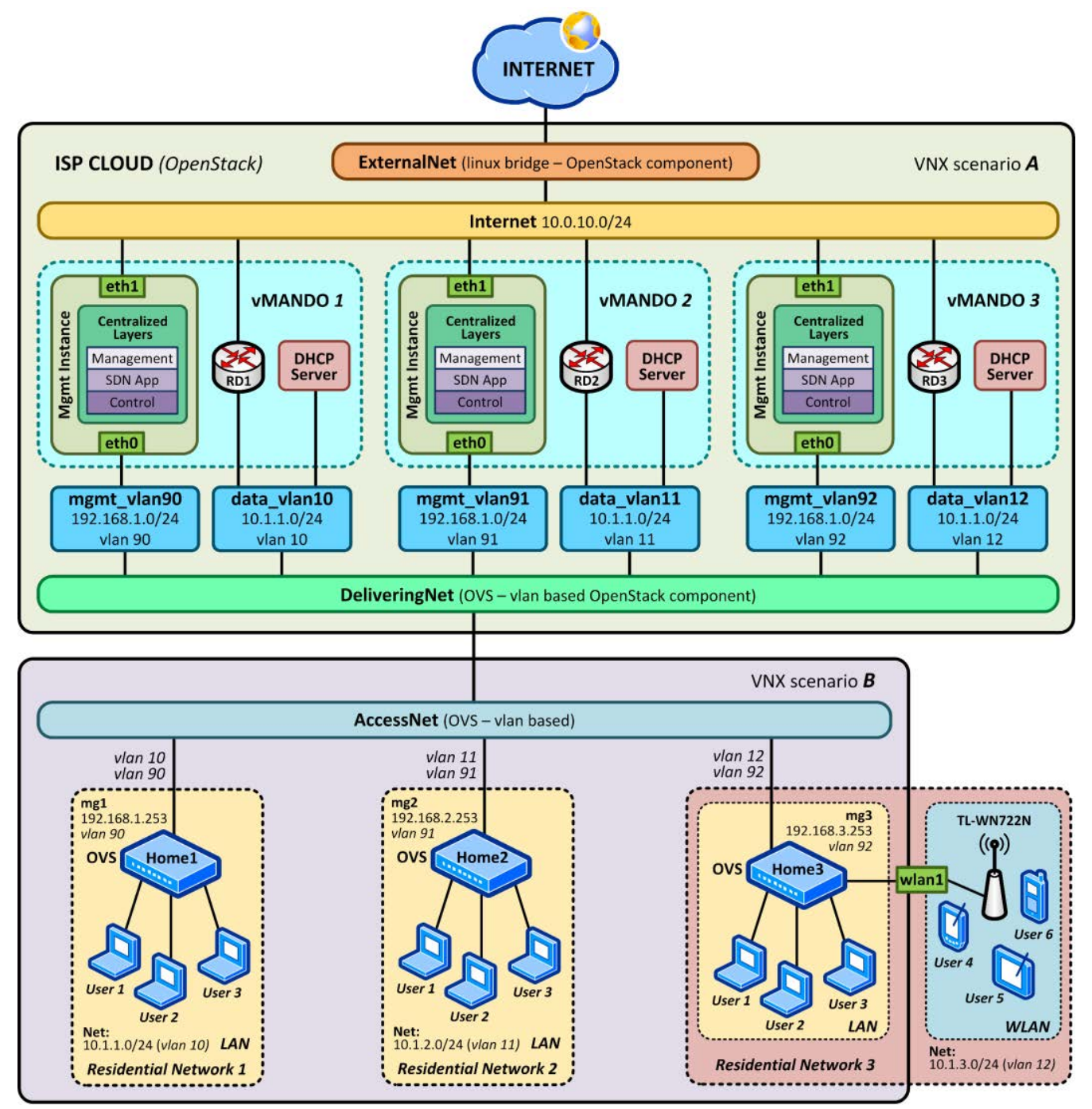

Figura 5.8 Segunda versión del banco de pruebas virtual desarrollado para la prueba de concepto. La Nube del ISP se sustenta principalmente en OpenStack.

\footnotetext{
${ }^{10} \mathrm{https}$ //wireless.wiki.kernel.org/en/users/documentation/hostapd

${ }^{11} \mathrm{http}: / /$ web.dit.upm.es/vnxwiki/index.php/Vnx-labo-openstack-4nodes-basic-liberty
} 
De acuerdo con la sección 3.3.1, a cada red residencial se le proporciona un vMANDO en la Nube del ISP. Cada vMANDO está compuesto por los componentes SDN y NFV. El componente SDN, representado por la Instancia de Gestión, se desplegó en una máquina virtual, mientras que el componente NFV, representado por el encaminamiento, se implementó utilizando Neutron de OpenStack. Para aislar el tráfico de Gestión y Datos, se definieron dos VLANs por cada red residencial. En la Nube del ISP, cada vMANDO está provisto con dos redes virtuales basadas en VLAN que se conectaron directamente a la infraestructura de red en la Nube (DeliveringNet) aplicando el concepto de "redes de proveedores". La red virtual basada en VLAN asociada al tráfico de datos incluye un servidor DHCP para configurar los dispositivos conectados a la red residencial. Similarmente, se desplegó la red virtual "Internet" para proporcionar a cada vMANDO de conectividad a Internet.

El escenario de VNX $B$ se usa para emular tres diferentes redes residenciales y la red de acceso correspondiente. Al igual que en la primera versión del banco de pruebas virtual, en las instalaciones del usuario residencial, la capa de infraestructura (RGW basado en SDN) se implementa utilizando Open vSwitch v2.4.0 (OVS). Cada red residencial está compuesta de varias máquinas virtuales que representan dispositivos de usuario y se habilita una WLAN a través del punto de acceso que utiliza el daemon hostapd.

\subsubsection{Pruebas de Desempeño}

El retraso en la instalación de la entrada de flujo (flow entry installation delay) representa uno de los principales temas de interés al momento de proponer una solución basada en SDN. Considerando que se utiliza un control en banda para el RGW basado en SDN, este retraso depende de diferentes factores como la latencia de la red de acceso, la latencia de la red del ISP, el tiempo de procesamiento del sistema de gestión, el ancho de banda disponible de la conexión a Internet, la carga de la plataforma en la Nube, la carga de tráfico, la calidad del software del dispositivo que soporta OpenFlow, las tecnologías de virtualización, etc. Un aumento excesivo de este retraso podría crear un cuello de botella que representa un problema de escalabilidad. Como resultado, el plano de datos envía continuamente paquetes de control, sobrecargando la conexión a Internet y poniendo en peligro la capacidad de respuesta en tiempo real del plano de control. En comparación con un RGW "todo en uno", dicho retardo de instalación de entrada de flujo es prácticamente insignificante ya que los planos de control y datos coexisten en el mismo dispositivo de red.

Con el objetivo de verificar la factibilidad de desplegar el plano de control del RGW basado en SDN en la Nube del ISP, como se muestra en la Figura 5.6, el retraso en la instalación de la entrada de flujo se mide considerando la carga de tráfico de la conexión a Internet, el ancho de banda de la conexión a Internet, la latencia de la red de acceso y el tiempo de procesamiento del sistema de gestión. Para este propósito, se utilizó el banco de pruebas virtual de la Figura 5.7 y se definieron tres intervalos de tiempo como se muestra en la Figura 5.9. De esta manera, el tiempo que requiere un paquete para alcanzar el sistema de gestión se representa por $\beta_{1}$, el tiempo para procesar un Packet-In y enviar un flow-mod se representa por $\beta_{2}$ y el tiempo que requiere un flow-mod para alcanzar el RGW basado en SDN se representa por $\beta_{3}$. Para obtener el retraso en la instalación de la entrada de flujo, estos tres intervalos de tiempo se suman. Además, como se establece una comunicación bidireccional, este retraso se midió para los flujos ascendente (upstream) y descendente (downstream).

Considerando que el objetivo es observar el comportamiento en las peores condiciones, se utilizó la herramienta Iperf ${ }^{12}$ para emular diferentes cargas de tráfico a cargo de congestionar la dirección ascendente. En consecuencia, la congestión del tráfico dificulta que el tráfico de control llegue al sistema de gestión (cuello de botella). Con el fin de proporcionar un escenario realista, se determinó el número de usuarios residenciales con base en el hogar estadounidense promedio en 2016, que consta de 2,53 personas $^{13}$. Este valor se redondeó a 3 y posteriormente se duplicó para empeorar la congestión del tráfico. Por lo tanto, 6 usuarios formaron parte del escenario de prueba, cada uno con un dispositivo de red a cargo de ejecutar una conexión Iperf. Para completar el escenario de prueba y cumpliendo con el objetivo de observar el comportamiento en las peores condiciones, se configuró la conexión a Internet

\footnotetext{
12 https://iperf.fr/

${ }^{13} \mathrm{https} / / /$ www.statista.com/statistics/183648/average-size-of-households-in-the-us/
} 
con un ancho de banda simétrico de 20 Mbps y se configuró la red de acceso con una latencia promedio (ida y vuelta) de 34,9 ms considerando la hora punta [109].

La prueba consistió en incrementar la carga de tráfico mediante el incremento del número de conexiones Iperf establecidas (de una a seis). Para cada carga de tráfico, se midió el retraso en la instalación de la entrada de flujo de un nuevo flujo de tráfico. La medición se realizó con base en las marcas de tiempo de los paquetes capturados en la interfaz de gestión del RGW basado en SDN (mg1) y la interfaz del sistema de gestión (VLAN 10). Considerando al retraso en la instalación de la entrada de flujo como una variable aleatoria, su valor medio se estimó mediante el cálculo de la media muestral para diferentes cargas de tráfico. Para este fin, la prueba de una carga de tráfico específica se repitió 20 veces para calcular su media muestral correspondiente.

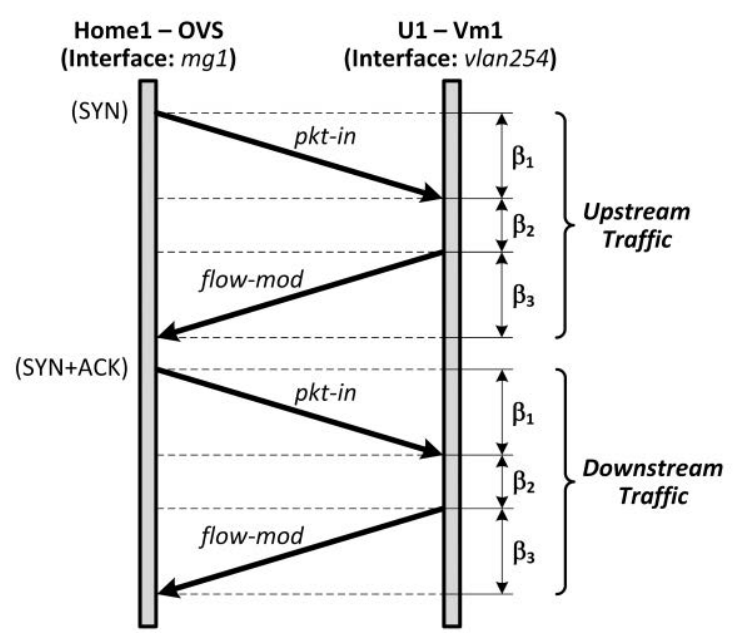

Figura 5.9 Intervalos de tiempo que intervienen en la instalación de una entrada de flujo. La sumatoria de estos intervalos permite obtener el retraso en la instalación de la entrada de flujo.

La Figura 5.10 muestra los tres intervalos de tiempo definidos $\left(\beta_{1}, \beta_{2}\right.$ y $\left.\beta_{3}\right)$ en función de las diferentes cargas de tráfico. Como se muestra en la figura, el valor del intervalo de tiempo $\beta_{1}$ aumenta a medida que aumenta la carga de tráfico. Este comportamiento se observa tanto para el tráfico ascendente como descendente. Teniendo en cuenta que la conexión a Internet está completamente congestionada y que la red de acceso experimenta una latencia de hora punta de $34,9 \mathrm{~ms}$, un Packet-In de un flujo de tráfico ascendente requiere $1282 \mathrm{~ms}$ para alcanzar el sistema de gestión mientras que un Packet-In de un flujo de tráfico descendente requiere $1094 \mathrm{~ms}$.

El intervalo de tiempo $\beta_{2}$ no se ve afectado por el aumento en la carga de tráfico y varía desde un valor mínimo de $0,725 \mathrm{~ms}$ hasta un valor máximo de $2,636 \mathrm{~ms}$, obteniendo un valor promedio de $1,337 \mathrm{~ms}$ y una desviación estándar de 0,573 . Este intervalo de tiempo presenta una variabilidad significativa que depende de diferentes factores como el tiempo de procesamiento no determinista e individual de las diferentes capas de software que forman parte del sistema de gestión, el retraso introducido por las tecnologías de virtualización, así como la potencia computacional de la Nube del ISP.

El intervalo de tiempo de $\beta_{3}$ se ve afectado principalmente por la latencia configurada en la red de acceso. Como el aumento en la carga de tráfico está destinado a congestionar la dirección ascendente, la dirección descendente está libre de congestión, solo se reciben los ACKs de las conexiones Iperf establecidas. Este intervalo de tiempo varía desde un valor mínimo de 17,466 ms hasta un valor máximo de $17,501 \mathrm{~ms}$, obteniendo un valor promedio de $17,479 \mathrm{~ms}$ y una desviación estándar de 0,01 . Considerando que la latencia de ida y vuelta de la red de acceso es $34,9 \mathrm{~ms}$, las mediciones obtenidas se corresponden aproximadamente a la latencia unidireccional (17,45 ms). Como el intervalo $\beta_{3}$ no se ve afectado por factores adicionales distintos de la latencia de la red de acceso unidireccional, presenta una variabilidad menor en comparación con el intervalo $\beta_{2}$. 
El retraso en la instalación de la entrada de flujo se obtiene sumando los tres intervalos de tiempo para cada carga de tráfico como se muestra en la Figura 5.10. Como muestran los resultados, el incremento en la carga de tráfico de la conexión a Internet incrementa significativamente el retraso en la instalación de la entrada de flujo. En el peor de los casos, se requieren $1301 \mathrm{~ms}$ para instalar una nueva entrada de flujo en el RGW basado en SDN lo cual indica que no es factible implementar el sistema de gestión en la Nube del ISP.
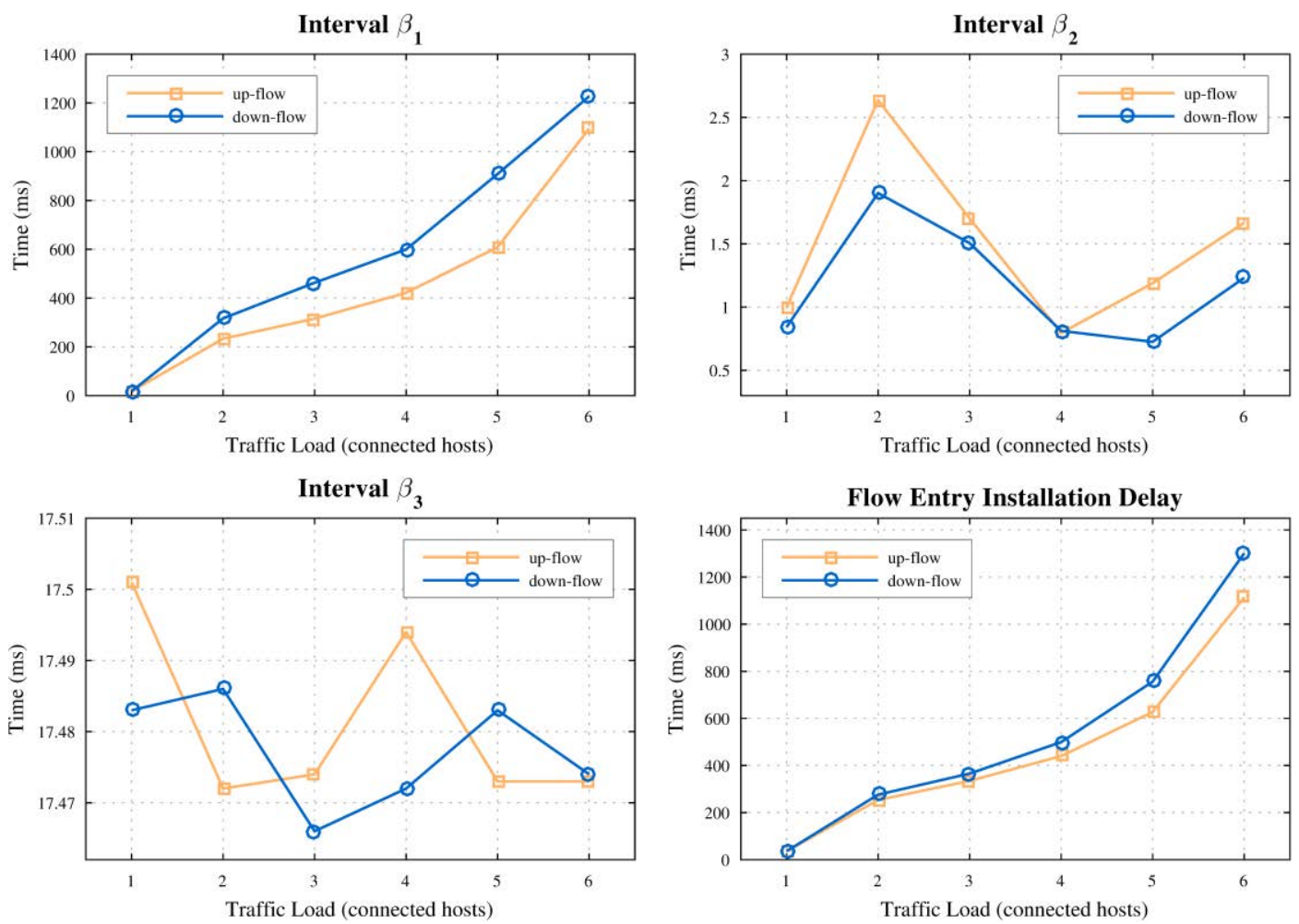

Figura 5.10 El retraso en la instalación de la entrada de flujo como una función de la carga de tráfico. Para congestionar la red de acceso se ejecuta una conexión iperf por host conectado.

Con base en los resultados de la prueba, un enfoque de gestión basado en la Nube requiere la provisión de un canal de comunicación dedicado para el tráfico de gestión con el fin de aislarlo de la congestión del tráfico. En este sentido, la conexión a Internet de cada red residencial es provista con dos enlaces virtuales, el primero para el tráfico de gestión y el segundo para el tráfico de datos. Este requisito se implementó en el banco de pruebas virtual utilizando la disciplina de colas Hierarchical Token Bucket (HTB). El ancho de banda de la conexión a Internet $(20 \mathrm{Mbps})$ se distribuyó entre los dos canales virtuales definidos. Arbitrariamente, el canal para el tráfico de gestión se configuró con $1 \mathrm{Mbps}$, mientras que el canal para el tráfico de datos se configuró con $19 \mathrm{Mbps}$. Una vez que se configuraron los canales virtuales, se repitieron las pruebas anteriores para observar el comportamiento resultante.

En general, la provisión de canales virtuales dedicados aisló con éxito el tráfico de gestión de la congestión del tráfico de datos, mejorando significativamente el comportamiento del retraso en la instalación de la entrada de flujo. Los resultados de la prueba se muestran en la Figura 5.11. Como el tráfico de gestión es provisto con un canal dedicado, la condición de congestión causada por el incremento en la carga de tráfico no afectó al intervalo de tiempo $\beta_{1}$. Los intervalos de tiempo $\beta_{1}$ y $\beta_{3}$ fueron afectados solamente por la latencia de la red de acceso unidireccional, obteniéndose un valor promedio de $17,491 \mathrm{~ms}$ y $17,482 \mathrm{~ms}$ respectivamente. El intervalo de tiempo $\beta_{2}$ mostró un comportamiento similar en comparación con la prueba anterior (Figura 5.10), obteniendo un valor promedio de 1,013 ms para procesar Packet-Ins. 

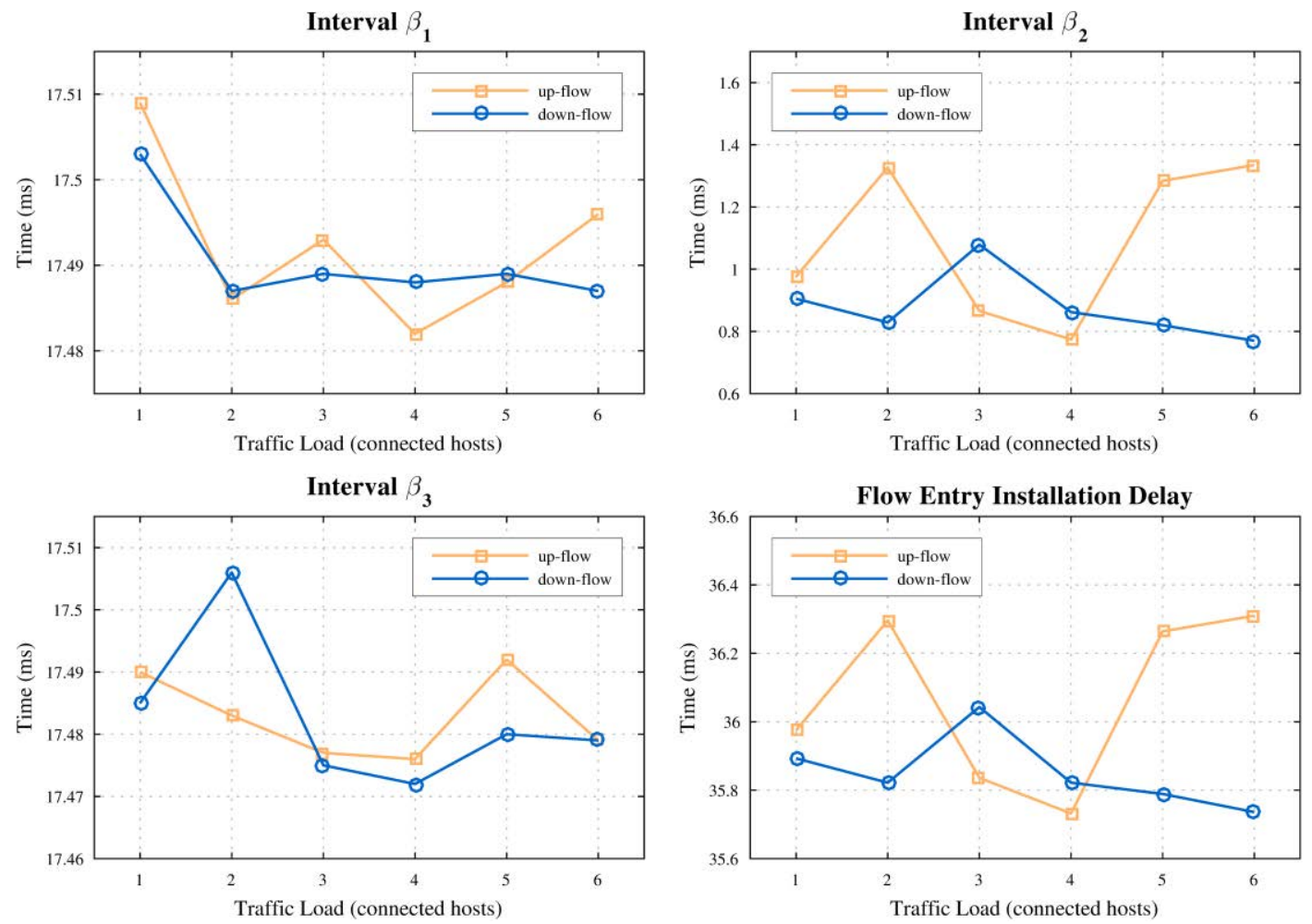

Figura 5.11 Comportamiento del retraso en la instalación de la entrada de flujo utilizando canales virtuales. La mejora es notable ya que la latencia de la red de acceso solamente afecta a $\beta_{1}$ y el retraso total permanece constante a medida que aumenta la carga de tráfico.

En una experimentación adicional, el canal virtual del tráfico de gestión se configuró con diferentes valores de ancho de banda con el fin de determinar, empíricamente, el valor mínimo que se requiere para establecer una comunicación adecuada entre el RGW basado en SDN y el sistema de gestión. En el experimento se determinó que se requiere un ancho de banda mínimo de $14 \mathrm{Kbps}$. Considerando este valor de ancho de banda, el tráfico de gestión es prácticamente insignificante en comparación con el tráfico de datos. Este resultado es muy significativo porque el canal virtual del tráfico de gestión se puede configurar para usar un porcentaje mínimo del ancho de banda de la conexión a Internet (como se mostró en el experimento anterior). Por el contrario, para no utilizar un porcentaje del ancho de banda de la conexión a Internet, se puede asignar un ancho de banda adicional para el tráfico de gestión. En cualquier caso, la asignación de un canal virtual dedicado para el tráfico de gestión no tiene efectos negativos debido a que se requiere un valor prácticamente insignificante de ancho de banda.

\subsubsection{Pruebas de provisión de QoS}

Como se explicó en la sección 5.2.1, el campo de prioridad del servicio de una política permite la configuración de un nivel de QoS. Para probar el funcionamiento de esta funcionalidad, se implementó un servicio de Video bajo Demanda (VoD) en el banco de pruebas virtual de la Figura 5.8 mediante "Dynamic Adaptive Streaming over HTTP" (DASH). Con base en la propuesta presentada por Lentisco et al. [110], se codificó el conocido video de animación "Big Buck Bunny" utilizando H.264 y velocidad de bits variable (VBR) con una resolución de 720p. Se crearon cuatro representaciones o calidades del video utilizando las siguientes cuatro tasas de bits de codificación promedio ( $\mathrm{kbps}$ ): $\mathrm{VQ}_{1}=250, \mathrm{VQ}_{2}=$ $500, \mathrm{VQ}_{3}=975 \mathrm{y} \mathrm{VQ}=1600$. Un servidor de contenido, desplegado en la Nube del ISP como otra VM dentro de OpenStack, se encargó de almacenar y entregar estas diferentes calidades del video a los clientes. Los clientes utilizaron el software DASH-JS v1.6.0 $0^{14}$ para recuperar y reproducir el video. El

\footnotetext{
${ }^{14}$ https://github.com/Dash-Industry-Forum/dash.js/releases/tag/v1.6.0
} 
cliente DASH se encarga de estimar el ancho de banda disponible y elegir la calidad del video que debe recuperarse para cada segmento.

Para probar la efectividad de las capacidades de provisión de QoS de NERON, se comparó la variación de las calidades del video recuperadas por el cliente DASH entre la no provisión de QoS y la provisión de QoS. Para analizar el comportamiento en el peor de los casos, la red de acceso se configuró con un ancho de banda simétrico típico de 20 Mbps y una latencia promedio (ida y vuelta) de $29 \mathrm{~ms}$ considerando la hora punta [111]. Se conectaron cuatro dispositivos por cable a la red residencial. En uno de ellos se ejecutó el cliente DASH-JS y en los tres restantes se ejecutaron clientes FTP (File Transfer Protocol) para descargar un archivo de gran tamaño desde un servidor FTP ubicado en la Nube del ISP y congestionar la conexión a Internet. Una vez que se ha iniciado la descarga en todos los clientes FTP, el cliente DASH inició la reproducción del video la cual duró 150 segundos.

Para la provisión de QoS y considerando la descripción de NERON de la sección 5.2.1, se configuró cada puerto activo del RGW habilitado para OpenFlow con tres colas diferentes (q1, q2 y q3) las cuales se asignan a una prioridad del servicio (baja, media y alta). El ancho de banda total por puerto se distribuye entre estas colas utilizando los siguientes porcentajes: $q 1$ con $15 \%, q 2$ con $35 \%$ y $q 3$ con $50 \%$. El ancho de banda total por puerto se obtiene al dividir el ancho de banda de la conexión a Internet entre el número de puertos activos. Con base en los parámetros de la prueba, con un ancho de banda de conexión a Internet de 20 Mbps y cuatro puertos activos, se realiza una asignación de $0,75 \mathrm{Mbps}$ a la prioridad del servicio baja (q1), 1,75 Mbps a la prioridad del servicio media (q2) y 2,5 Mbps a la prioridad del servicio alta (q3). Esto representa un mecanismo simple de QoS para fines demostrativos.

Al no disponer de capacidades de provisión de QoS, la Figura 5.12 muestra que durante la prueba la calidad del video experimentó una alta variabilidad. Este comportamiento fue el resultado de la congestión presente en la conexión a Internet causada por el tráfico FTP y el de video. La Figura 5.12 (a) muestra que la conexión TCP del cliente DASH intenta obtener la mayor parte del ancho de banda disponible en ausencia de un mecanismo adicional que asigne eficientemente este recurso de red. Con respecto a la distribución de las calidades del video, la Figura 5.12 (b) muestra que el $46.53 \%$ del tiempo de duración de la prueba, la calidad más solicitada fue la calidad del video más baja.

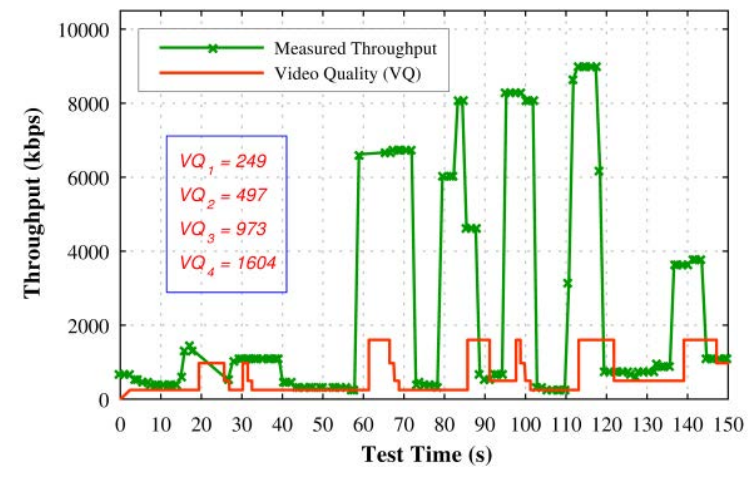

(a)

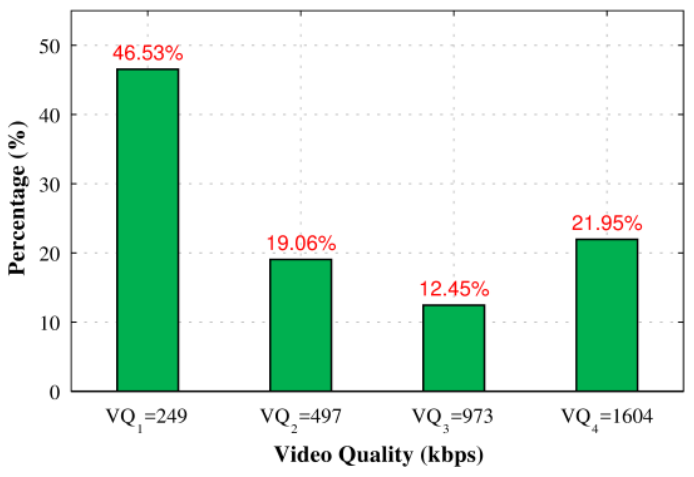

(b)

Figura 5.12 Variabilidad en la calidad del video en el cliente DASH-JS sin provisión de QoS: (a) Caudal de tráfico y calidades del video medidas, (b) Distribución de las calidades del video durante la prueba.

Al disponer de las capacidades de provisión de QoS de NERON, se definió una política con una prioridad del servicio alta para el dispositivo final que ejecuta el cliente DASH y los tres dispositivos que ejecutan los clientes FTP, utilizando para ello el cliente de la aplicación de Control Parental. Con base en los parámetros de la prueba, se asignó la cola q3 (2,5 Mbps) al cliente DASH y los clientes FTP y se distribuyó el ancho de banda restante entre las colas q1 (0,75 Mbps) y q2 (1,75 Mbps). De esta manera, en otro escenario de prueba, se pueden asignar las colas restantes a otros servicios que se ejecutan en el mismo dispositivo final. 
La Figura 5.13 (a) muestra que la variabilidad en la calidad del video disminuyó en comparación con la Figura 5.12 (a). La provisión de QoS permitió que el 67,92\% del tiempo de duración de la prueba, la calidad del video más alta fuese la calidad más solicitada, tal y como se muestra en la Figura 5.13 (b). El resultado obtenido es alentador considerando que sin provisión de QoS la calidad del video más alta fue solicitada el $21,95 \%$ del tiempo de duración de la prueba. Este sencillo ejemplo muestra cómo las capacidades de provisión de QoS de NERON permiten mejorar un servicio de VoD y permiten verificar la factibilidad y ventajas de la gestión de red basada en aplicaciones.

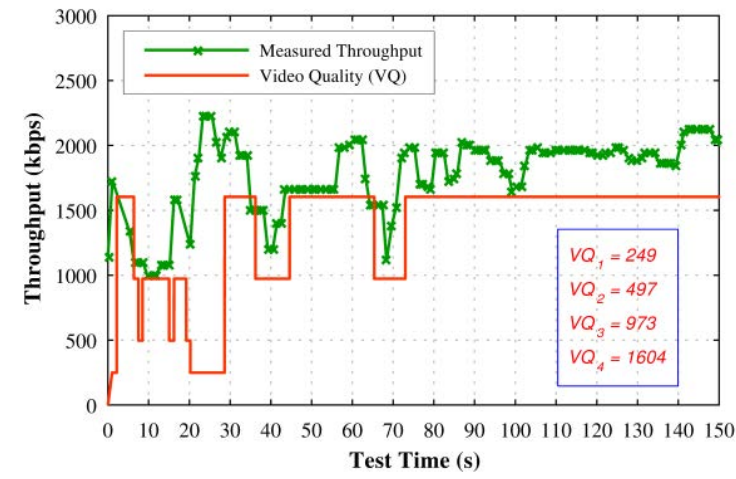

(a)

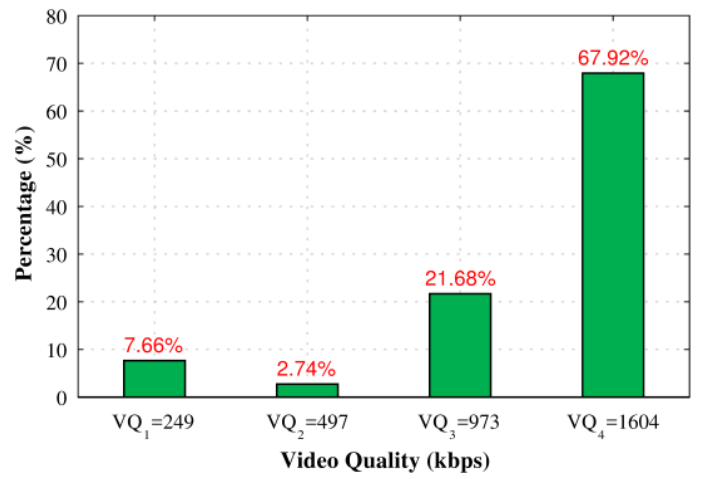

(b)

Figura 5.13 Variabilidad en la calidad del video en el cliente DASH-JS con provisión de QoS. (a) Caudal de tráfico y calidades del video medidas, (b) Distribución de las calidades del video durante la prueba.

\subsection{Implementación del Tratamiento de Tráfico Diferenciado}

En esta segunda parte del capítulo, tal y como se indicó en la sección introductoria, se aborda la implementación del tratamiento de tráfico diferenciado presentada en el capítulo 4. Para este fin, en primer lugar, se define en detalle el conjunto de operaciones que incorpora el API de la aplicación de Tratamiento de Tráfico considerando como ejemplo el trabajo realizado en la definición del API REST para las aplicaciones de Control Parental y de Estado de la Red presentadas en la sección 5.3.1.

En segundo lugar, se desarrolla el proceso para implementar vMANDO como un Servicio de Red para lo cual se obtiene el grafo de Topología de Conectividad de Red (NCT) necesario para identificar los elementos de información que describen el servicio de red y sus componentes. Es importante mencionar que, por el momento, en la tesis se ha desarrollado la implementación del tratamiento de tráfico diferenciado desde una perspectiva de diseño, es decir, falta desarrollar la aplicación real y experimentar con una plataforma NFV como Tacker.

\subsubsection{API de la aplicación de Tratamiento de Tráfico}

En la sección 4.4.4, se definió de forma general el API REST de la aplicación de Tratamiento de Tráfico. Para implementar esta aplicación es indispensable definir en detalle el conjunto de operaciones que el API proporcionará. De esta manera, la Tabla 5.7 resume el API de la aplicación y detalla los URIs definidos, los métodos HTTP utilizados y la descripción de la operación que se proporciona.

Es importante recordar que el API está encargado de controlar los procesos CRUD de los objetos que utiliza la aplicación: flujo de tráfico, contenedor de tráfico, VNF y SFC. De esta manera, la Tabla 5.8, la Tabla 5.9, la Tabla 5.10 y la Tabla 5.11 permiten completar el proceso de definición al mostrar los parámetros de solicitud y respuesta del API REST para cada objeto utilizado. 
Tabla 5.7 API REST proporcionada por la aplicación de Tratamiento de Tráfico. El API incorpora operaciones CRUD para flujos de tráfico, contenedores de tráfico, SFCs y VNFs.

\begin{tabular}{|c|c|c|}
\hline URI & Método & API \\
\hline /traffictreatment/flows & GET & List traffic flows: lists traffic flows created \\
\hline$/$ traffictreatment/flows $/<$ traffic_flow_id $>$ & GET & $\begin{array}{l}\text { Show traffic flow: shows information of a specific } \\
\text { traffic flow }\end{array}$ \\
\hline /traffictreatment/flows & POST & $\begin{array}{l}\text { Create traffic flow: creates a traffic flow by } \\
\text { providing the match criterion, name and } \\
\text { description. The match criterion may be simple or } \\
\text { compound, considering, for instance, physical port, } \\
\text { device's MAC address, IP address destination or } \\
\text { TCP port destination }\end{array}$ \\
\hline$/$ traffictreatment/flows $/<$ traffic_flow_id $>$ & PUT & $\begin{array}{l}\text { Update traffic flow: updates the values of certain } \\
\text { attributes of the traffic flow }\end{array}$ \\
\hline$/$ traffictreatment/flows $/<$ traffic_flow_id $>$ & DELETE & Delete traffic flow: deletes a specific traffic flow \\
\hline /traffictreatment/containers & GET & $\begin{array}{l}\text { List traffic containers: lists traffic containers } \\
\text { created }\end{array}$ \\
\hline /traffictreatment/containers $<$ container_id $>$ & GET & $\begin{array}{l}\text { Show traffic container: shows information of a } \\
\text { specific traffic container }\end{array}$ \\
\hline /traffictreatment/containers & POST & $\begin{array}{l}\text { Create traffic container: creates a traffic container } \\
\text { by providing the traffic flow(s) to be part of the } \\
\text { container, bandwidth to be allocated, name and } \\
\text { description }\end{array}$ \\
\hline$/$ traffictreatment/containers $<$ container_id $>$ & PUT & $\begin{array}{l}\text { Update traffic container: updates the values of } \\
\text { certain attributes of the traffic container }\end{array}$ \\
\hline$/$ traffictreatment/containers $<$ container_id $>$ & DELETE & $\begin{array}{l}\text { Delete traffic container: deletes a specific traffic } \\
\text { container }\end{array}$ \\
\hline$/$ traffictreatment/sfcs & GET & List SFCs: lists SFCs created \\
\hline$/$ traffictreatment $/$ sfes $/<$ sfc_id $>$ & GET & Show SFC: shows information of a specific SFC \\
\hline$/$ traffictreatment/sfcs & POST & $\begin{array}{l}\text { Create } S F C \text { : creates a SFC by providing the VNF(s) } \\
\text { to be included in the chain, name, description and } \\
\text { the traffic container Id the SFC will be applied to }\end{array}$ \\
\hline$/$ traffictreatment $/$ sfcs $/<$ sfc_id $>$ & PUT & $\begin{array}{l}\text { Update } S F C \text { : updates the values of certain attributes } \\
\text { of the SFC }\end{array}$ \\
\hline$/$ traffictreatment $/$ sfcs $/<$ sfc_id $>$ & DELETE & Delete $S F C$ : deletes a specific SFC \\
\hline /traffictreatment/vnfs & GET & $\begin{array}{l}\text { List VNFs: lists the available VNFs on the NFV } \\
\text { platform }\end{array}$ \\
\hline$/$ traffictreatment $/$ vnfs $/<$ vnf_id $>$ & GET & Show VNF: shows information of a specific VNF \\
\hline$/$ traffictreatment/vnfs $/<$ vnf_id $>$ & PUT & $\begin{array}{l}\text { Update VNF config: updates the VNF's } \\
\text { configuration parameters }\end{array}$ \\
\hline$/$ traffictreatment/vnfs/restore/ $<$ vnf_id $>$ & GET & $\begin{array}{l}\text { Restore VNF config: restores the default VNF's } \\
\text { configuration parameters }\end{array}$ \\
\hline
\end{tabular}


Tabla 5.8 Parámetros de Solicitud y Respuesta del API REST para las operaciones CRUD de flujos de tráfico.

\begin{tabular}{|c|c|c|c|c|}
\hline \multirow{2}{*}{ API } & \multicolumn{2}{|c|}{ Parámetros de Solicitud } & \multicolumn{2}{|c|}{ Parámetros de Respuesta } \\
\hline & Nombre & Tipo & Nombre & Tipo \\
\hline List traffic flows & none & none & traffic_flows_ids & array \\
\hline \multirow{7}{*}{ Show traffic flow } & \multirow{7}{*}{ traffic_flow_id } & \multirow{7}{*}{ string } & traffic_flow_id & string \\
\hline & & & match_criterion & array \\
\hline & & & container_id_allocated & string \\
\hline & & & name & string \\
\hline & & & description & string \\
\hline & & & creation_date & string \\
\hline & & & update_date & string \\
\hline \multirow{7}{*}{ Create traffic flow } & \multirow{2}{*}{ match_criterion } & \multirow{2}{*}{ array } & traffic_flow_id & string \\
\hline & & & match_criterion & array \\
\hline & \multirow{2}{*}{ name } & \multirow{2}{*}{ string } & container_id_allocated & string \\
\hline & & & name & string \\
\hline & \multirow{3}{*}{ description } & \multirow{3}{*}{ string } & description & string \\
\hline & & & creation_date & string \\
\hline & & & update_date & string \\
\hline \multirow{7}{*}{ Update traffic flow } & \multirow{2}{*}{ traffic_flow_id } & \multirow{2}{*}{ string } & traffic_flow_id & string \\
\hline & & & match_criterion & array \\
\hline & \multirow{2}{*}{ match_criterion } & \multirow{2}{*}{ array } & container_id_allocated & string \\
\hline & & & name & string \\
\hline & \multirow{2}{*}{ name } & \multirow{2}{*}{ string } & description & string \\
\hline & & & creation_date & string \\
\hline & description & string & update_date & string \\
\hline Delete traffic flow & traffic_flow_id & string & result & string \\
\hline
\end{tabular}

Tabla 5.9 Parámetros de Solicitud y Respuesta del API REST para las operaciones CRUD de contenedores de tráfico.

\begin{tabular}{|c|c|c|c|c|}
\hline \multirow{2}{*}{ API } & \multicolumn{2}{|c|}{ Parámetros de Solicitud } & \multicolumn{2}{|c|}{ Parámetros de Respuesta } \\
\hline & Nombre & Tipo & Nombre & Tipo \\
\hline List traffic containers & None & None & traffic_containers_ids & array \\
\hline \multirow{9}{*}{ Show traffic container } & \multirow{9}{*}{ traffic_container_id } & \multirow{9}{*}{ string } & traffic_container_id & string \\
\hline & & & traffic_flows_ids & array \\
\hline & & & vlan_tag_allocated & integer \\
\hline & & & bw_allocated & integer \\
\hline & & & sfc_id_associated & string \\
\hline & & & name & string \\
\hline & & & description & string \\
\hline & & & creation_date & string \\
\hline & & & update_date & string \\
\hline \multirow{5}{*}{ Create traffic container } & \multirow{2}{*}{ traffic_flows_ids } & \multirow{2}{*}{ array } & traffic_container_id & string \\
\hline & & & traffic_flows_ids & array \\
\hline & \multirow{2}{*}{ bw_allocated } & \multirow{2}{*}{ integer } & vlan_tag_allocated & integer \\
\hline & & & bw_allocated & integer \\
\hline & name & string & sfc_id_associated & string \\
\hline
\end{tabular}




\begin{tabular}{|c|c|c|c|c|}
\hline & & & name & string \\
\hline & \multirow{3}{*}{ description } & \multirow{3}{*}{ string } & description & string \\
\hline & & & creation_date & string \\
\hline & & & update_date & string \\
\hline \multirow{9}{*}{ Update traffic container } & \multirow{2}{*}{ traffic_container_id } & \multirow{2}{*}{ string } & traffic_container_id & string \\
\hline & & & traffic_flows_ids & array \\
\hline & \multirow{2}{*}{ traffic_flows_ids } & \multirow{2}{*}{ array } & vlan_tag_allocated & integer \\
\hline & & & bw_allocated & integer \\
\hline & \multirow{2}{*}{ bw_allocated } & \multirow{2}{*}{ integer } & sfc_id_associated & string \\
\hline & & & name & string \\
\hline & \multirow{2}{*}{ name } & \multirow{2}{*}{ string } & description & string \\
\hline & & & creation_date & string \\
\hline & description & string & update_date & string \\
\hline Delete traffic container & traffic_container_id & string & result & string \\
\hline
\end{tabular}

Tabla 5.10 Parámetros de Solicitud y Respuesta del API REST para las operaciones CRUD de SFCs.

\begin{tabular}{|c|c|c|c|c|}
\hline \multirow{2}{*}{ API } & \multicolumn{2}{|c|}{ Parámetros de Solicitud } & \multicolumn{2}{|c|}{ Parámetros de Respuesta } \\
\hline & Nombre & Tipo & Nombre & Tipo \\
\hline List SFCs & None & None & sfcs_ids & array \\
\hline \multirow{7}{*}{ Show SFC } & \multirow{7}{*}{ sfc_id } & \multirow{7}{*}{ string } & sfc_id & string \\
\hline & & & vnfs_ids & array \\
\hline & & & traffic_container_id & string \\
\hline & & & name & string \\
\hline & & & description & string \\
\hline & & & creation_date & string \\
\hline & & & update_date & string \\
\hline \multirow{7}{*}{ Create $S F C$} & \multirow{2}{*}{ vnfs_ids } & \multirow{2}{*}{ array } & sfc_id & string \\
\hline & & & vnfs_ids & array \\
\hline & \multirow{2}{*}{ traffic_container_id } & \multirow{2}{*}{ string } & traffic_container_id & string \\
\hline & & & name & string \\
\hline & \multirow{2}{*}{ name } & \multirow{2}{*}{ string } & description & string \\
\hline & & & creation_date & string \\
\hline & description & string & update_date & string \\
\hline \multirow{7}{*}{ Update SFC } & sfc_id & string & sfc_id & string \\
\hline & vnfs_ids & array & vnfs_ids & array \\
\hline & traffic_container_id & string & traffic_container_id & string \\
\hline & \multirow{2}{*}{ name } & \multirow{2}{*}{ string } & name & string \\
\hline & & & description & string \\
\hline & \multirow{2}{*}{ description } & \multirow{2}{*}{ string } & creation_date & string \\
\hline & & & update_date & string \\
\hline Delete SFC & sfc_id & string & result & string \\
\hline
\end{tabular}


Tabla 5.11 Parámetros de Solicitud y Respuesta del API REST para las operaciones CRUD de VNFs.

\begin{tabular}{|c|c|c|c|c|}
\hline \multirow{2}{*}{ API } & \multicolumn{2}{|c|}{ Request Parameters } & \multicolumn{2}{|c|}{ Response Parameters } \\
\hline & Name & Type & Name & Type \\
\hline List VNFs & None & None & vnfs_ids & array \\
\hline \multirow{7}{*}{ Show VNF } & \multirow{7}{*}{ vnf_id } & \multirow{7}{*}{ string } & vnf_id & string \\
\hline & & & config_parameters & object \\
\hline & & & sfc_id_associated & string \\
\hline & & & name & string \\
\hline & & & description & string \\
\hline & & & association_date & string \\
\hline & & & update_date & string \\
\hline \multirow{7}{*}{ Update VNF config } & \multirow{3}{*}{ vnf_id } & \multirow{3}{*}{ string } & vnf_id & string \\
\hline & & & config_parameters & object \\
\hline & & & sfc_id_associated & string \\
\hline & \multirow{4}{*}{ config_parameters } & \multirow{4}{*}{ object } & name & string \\
\hline & & & description & string \\
\hline & & & association_date & string \\
\hline & & & update_date & string \\
\hline \multirow{7}{*}{ Restore VNF config } & \multirow{7}{*}{ vnf_id } & \multirow{7}{*}{ string } & vnf_id & string \\
\hline & & & config_parameters & object \\
\hline & & & sfc_id_associated & string \\
\hline & & & name & string \\
\hline & & & description & string \\
\hline & & & association_date & string \\
\hline & & & update_date & string \\
\hline
\end{tabular}

\subsubsection{Implementación de vMANDO como un Servicio de Red}

Como se explicó en la sección 2.3.4, el paradigma NFV utiliza plantillas de despliegue o descriptores para especificar un determinado servicio de red (NS). Considerando que un servicio de red resulta de la composición de diferentes funciones de red virtualizadas, se puede establecer una relación de correspondencia entre un servicio de red y vMANDO. En la sección 2.3.2, se indicó que un NS incluye un grafo de topología de conectividad de red (NCT) y uno o más grafos de envío de VNF (VNFFGs). De esta manera, en una primera parte, se define el NCT para vMANDO con base en la estructura lógica mostrada en la Figura 4.4 de la sección 4.3.1.

La Figura 5.14 muestra la topología resultante. La red de Clasificación (Classification Network) es sustituida por el enlace virtual $V L 1$. El enlace virtual $V L 2$ está encargado de conectar las diferentes VNFs que se agregan a vMANDO. El enlace virtual $V L 3$ está encargado de agregar las conexiones procedentes de la Instancia de Gestión y de las VNFs para conectarlas a la red Externa (External Network) mientras que el enlace virtual VL4 proporciona conectividad hacia la red de Servicios (Services Network).

En el contexto de un servicio de red, la Instancia de Gestión es considerada como otra VNF más, con la variante de que contiene el Sistema de Gestión Centrado en el Usuario. Cada VNF se conecta a su respectivo enlace virtual mediante puntos de conexión $(\mathrm{CP})$, los cuales representan interfaces virtuales y/o físicas. Finalmente, $C P 01, C P 02$ y $C P 03$ actúan como puntos finales del servicio de red, en este caso del propio vMANDO. Así, cada vMANDO puede ser considerado como una VNF agregada con tres interfaces que deben ser conectadas a tres redes distintas para obtener el comportamiento requerido como se muestra en la parte derecha de la Figura 5.14. 
Finalmente, la posibilidad de crear diferentes VNFFGs o SFCs sobre el NCT que incluye un servicio de red, se utiliza en vMANDO para asociar una SFC específica a un contenedor de tráfico de la red residencial con el objetivo de proporcionar un tratamiento de tráfico diferenciado.

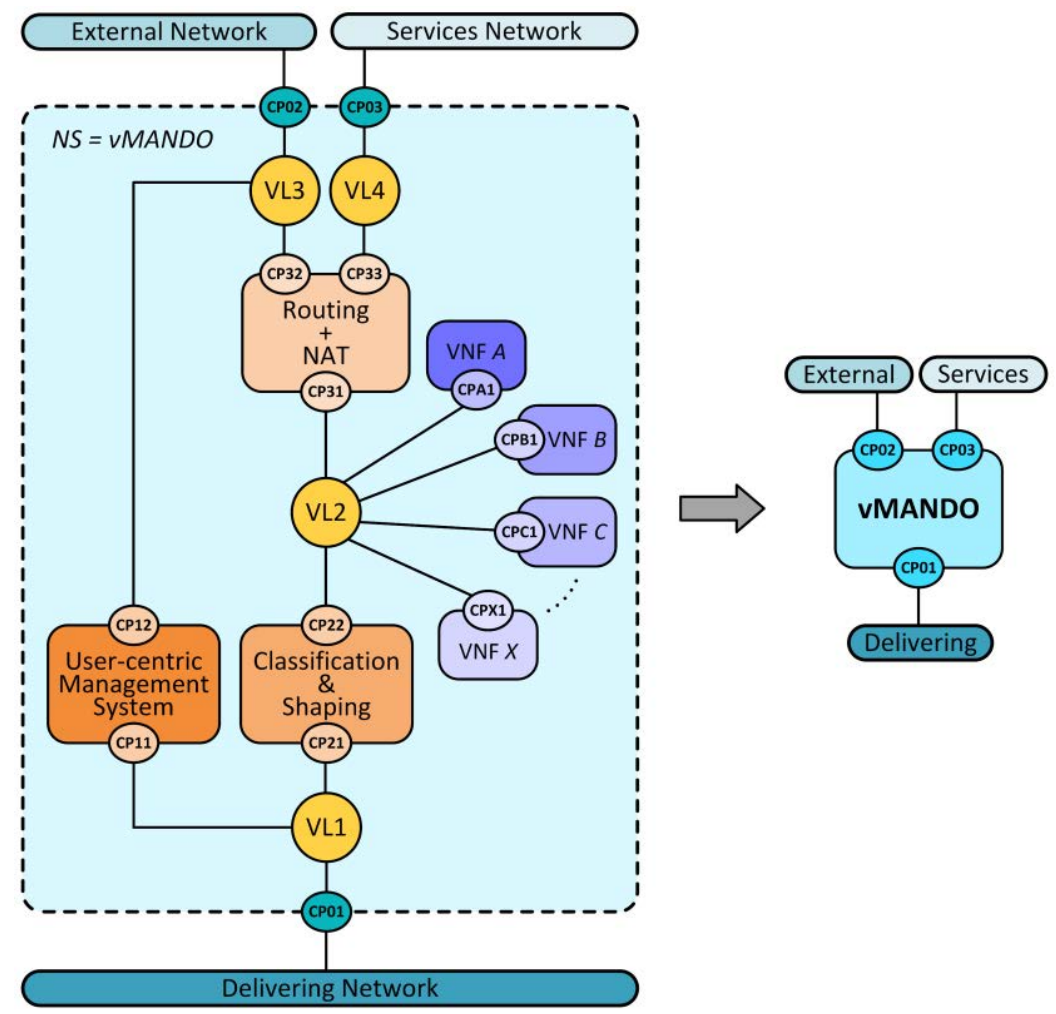

Figura 5.14 Grafo de topología de conectividad de red (NCT) para vMANDO.

\subsubsection{Especificación del Descriptor de vMANDO}

Una vez que el grafo de topología de conectividad de red para vMANDO ha sido definido, el siguiente paso consiste en especificar el descriptor correspondiente que será incorporado al catálogo del servicio de red de la plataforma de NFV con el objetivo de desplegar todos los componentes necesarios.

El descriptor de vMANDO, denominado como vMANDOD, sigue las directrices establecidas por TOSCA para especificar un NSD las cuales fueron explicadas en el apartado de Modelado de Elementos de Información de la sección 2.3.4. De esta manera, la Figura 5.15 muestra un ejemplo de vMANDOD escrito con YAML, en el cual se reconocen las VNFs, enlaces virtuales y puntos finales necesarios. Considerando que el perfil NFV de TOSCA incluye varias opciones adicionales para especificar en detalle los diferentes componentes de un servicio de red, se puede ampliar significativamente el descriptor para cumplir con determinados requisitos de funcionamiento. El descriptor incluye tres tipos principales de plantilla de nodos.

a. Las VNFs que componen vMANDO, siendo estas de dos tipos. Por una parte, están las VNFs fijas o por defecto que se incluyen y, por otra parte, están las VNFs adicionales o complementarias que se agregan bajo demanda. En conjunto, las VNFs fijas y adicionales permiten crear SFCs personalizadas con el objetivo de proporcionar un tratamiento de tráfico específico a un determinado contenedor de tráfico.

b. Los puntos finales o interfaces que permiten conectar cada vMANDO a las redes externas correspondientes.

c. Los enlaces virtuales que permiten conectar las diferentes VNFs entre sí y a su vez conectar vMANDO a las redes externas mediante los correspondientes puntos finales. 


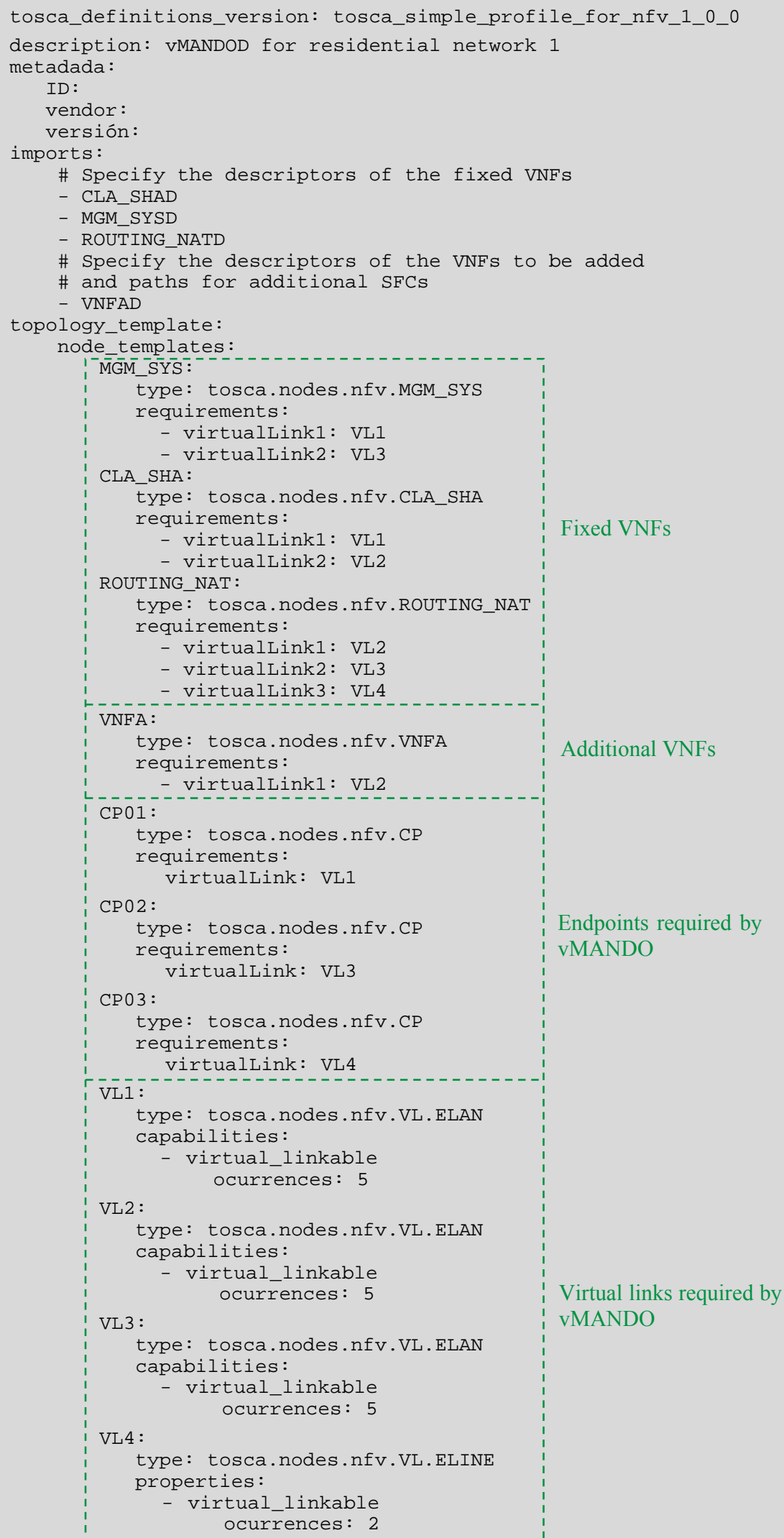

Figura 5.15 Descriptor para vMANDO (vMANDOD) basado en el NSD del perfil NFV de TOSCA. 
Es importante mencionar que todas las VNFs son descritas en detalle utilizando una plantilla de servicio independiente, mediante la especificación de un tipo de nodo sustituible. En la Figura 5.16 se muestra un ejemplo de un descriptor de VNF para implementar las funciones de Encaminamiento y NAT (Routing + NAT). La VNF se define como un nuevo tipo de nodo, especificando el tipo en el elemento tipos de nodo (node_types). Para este nuevo tipo de nodo se especifican los requerimientos de la VNF que serán proporcionados por el descriptor de vMANDO. Similarmente, se especifican las capacidades de la VNF que serán utilizadas por el descriptor de VNFFG. En el elemento mapeos de sustitución (substitution_mappings) se especifica el tipo de nodo sustituible definido por esta plantilla de servicio.

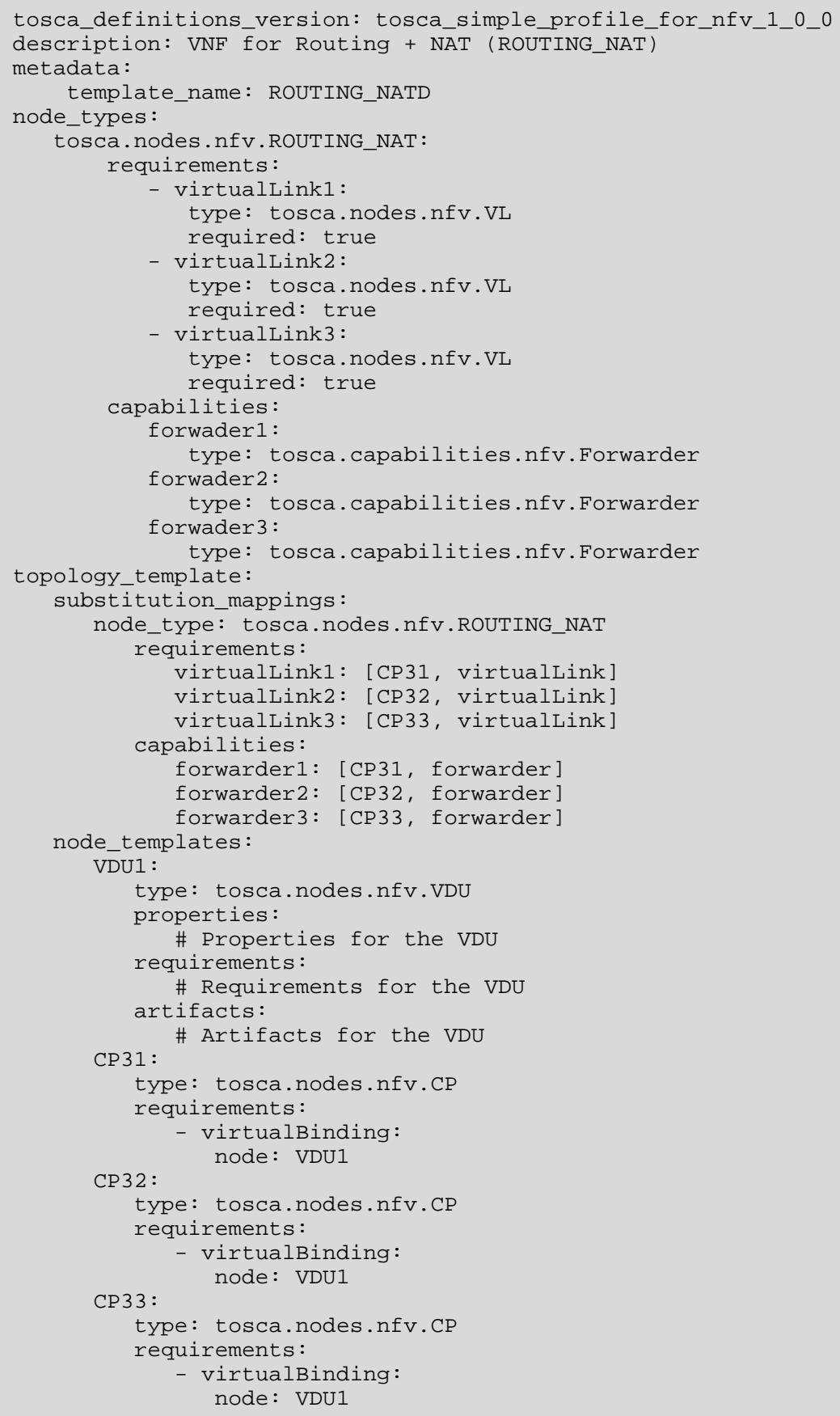

Figura 5.16 Descriptor para la VNF Routing + NAT. Se define un nuevo tipo de nodo para la VNF mediante una plantilla de servicio. 
Como se puede ver en la Figura 5.15, en la sección de plantillas de nodo (node_templates), al momento de incluir la VNF Routing_NAT, se especifica el correspondiente tipo de nodo previamente definido con el objetivo de proporcionarle los enlaces virtuales (VLs) requeridos. Los descriptores para el resto de VNFs siguen la misma estructura de la Figura 5.16. No obstante, cada VNF especifica sus propios puntos de conexión, los enlaces virtuales correspondientes y la funcionalidad que proporcionará de acuerdo con el grafo de topología de conectividad de red de la Figura 5.14. En el Apéndice B se incluye los descriptores de las VNFs restantes y se invita al lector a consultarlo para comprender de mejor manera el proceso de creación de descriptores.

Tanto el vMANDOD y los diferentes VNFDs permiten establecer la topología de conectividad de red de la Figura 5.14. Posteriormente, con el objetivo de proporcionar un tratamiento de tráfico específico a un contenedor de tráfico de la red residencial, es necesario especificar sobre dicha topología la cadena de función de servicio (SFC) o grafo de envío de VNF (VNFFG). Para este fin, se utiliza un VNFFGD donde se especifica principalmente dos aspectos relacionados al VNFFG como se muestra en la Figura 5.17. Por una parte, se especifica la ruta de envío (forwarding_path) que seguirá el tráfico a través de las distintas VNFs que componen vMANDO con base en una política de clasificación. Considerando el eventual uso de VLANs para la identificación de contenedores de tráfico, la política de clasificación utilizaría el Id de VLAN (vlan_id:10). El perfil NFV de TOSCA incorpora varias opciones para definir la política de clasificación, las cuales están detalladas en la documentación proporcionada. Por otra parte, se describe el propio VNFFG al enumerar los descriptores de las VNFs que lo constituyen (constituent_vnfs), así como los respectivos puntos de conexión (connection_point) y enlaces virtuales (dependent_virtual_link).

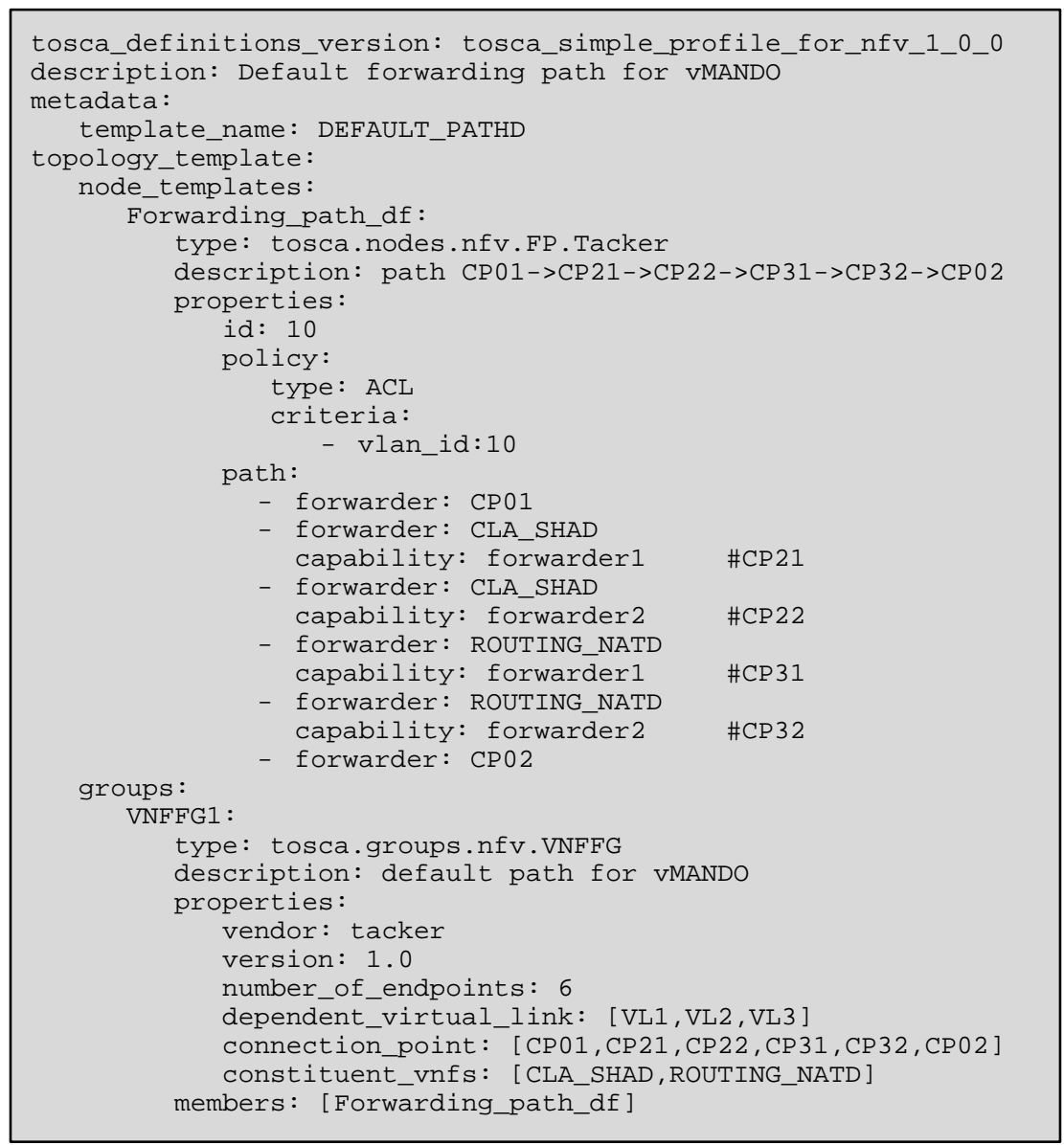

Figura 5.17 Descriptor para el VNFFG que especifica la ruta por defecto del tráfico de datos. El descriptor que se muestra está basado en los ejemplos ${ }^{15}$ que proporciona Tacker para el VNFFGD.

\footnotetext{
${ }^{15}$ https://github.com/openstack/tacker/tree/master/samples/tosca-templates
} 
En este punto, se podría resumir la implementación de los distintos componentes de vMANDO y sus relaciones mediante la Figura 5.18. Al utilizar TOSCA, la plantilla de servicio vMANDOD puede ser considerada como la plantilla principal y permite definir de forma global los diferentes componentes a ser desplegados. Los diferentes VNFDs son incluidos en la plantilla de servicio principal mediante un mapeo de sustitución, mientras que una ruta de envío a través de una determinada cadena de VNFs es especificada por un VNFFGD.

En conjunto, vMANDOD, VNFDs, VNFFGDs y VLDs permiten aplicar un tratamiento de tráfico específico a un contenedor de tráfico de la red residencial. Por otra parte, es importante mencionar que se podrían describir los puntos finales de vMANDO (CP01, CP02 y CP03 en la Figura 5.14) en plantillas de servicio independientes. El perfil NFV de TOSCA no considera esta opción, sin embargo, resultaría de gran utilidad y podría considerarse como un requisito funcional para optimizar la especificación de vMANDOD. De esta manera se incluiría una plantilla de servicio CPD como se muestra en la Figura 5.18 .

Con base en los detalles operacionales del tratamiento de tráfico diferenciado expuestos en la sección 4.5 , es importante mencionar que el despliegue inicial de una red residencial requiere la provisión de un descriptor por defecto de vMANDO en el cual se incluyen las VNFs fijas tal y como se muestra en la Figura 5.15. Una vez que se crea la instancia de vMANDO, el proceso posterior de creación de SFCs bajo demanda afecta tanto a la instancia de vMANDO que se encuentra en ejecución como al descriptor. Si bien la aplicación de Tratamiento de Tráfico utiliza las interfaces MANO para agregar VNFs a la instancia de vMANDO, el orquestador de NFV debería encargarse de crear una nueva versión del correspondiente descriptor en el catálogo y eliminar la versión previa una vez que se detiene la instancia.

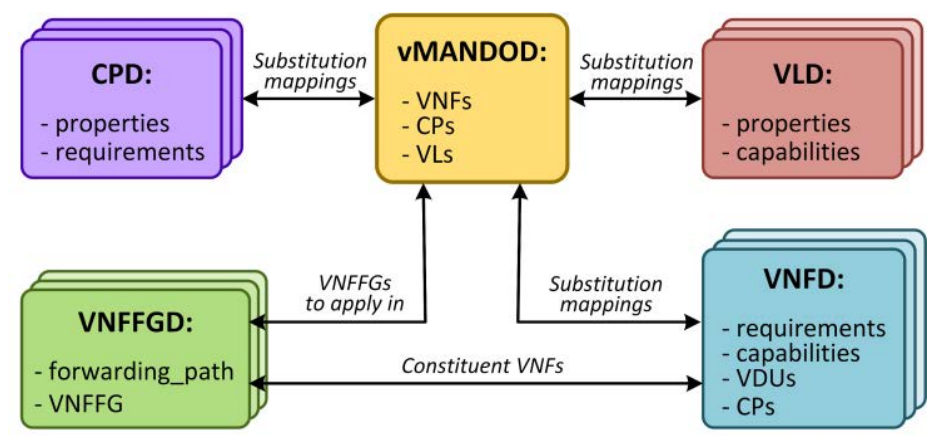

Figura 5.18 Relación entre las plantillas de servicio utilizadas para describir los componentes de vMANDO.

Finalmente, se podría decir que vMANDO al ser un caso específico dentro del caso general de despliegue de servicios de red, necesita que se especifiquen datos complementarios que permitan definir un modelo más completo lo cual permitiría ejecutar procesos de despliegue, monitorización, mantenimiento y corrección de fallos de forma más precisa. De esta manera, con base en el perfil NFV de TOSCA y considerando el API de orquestación de NFV de Tacker ${ }^{16}$, la Figura 5.19 (a) muestra la propuesta de un conjunto de datos ampliado que podría ser incluido ya sea en los "metadata" de vMANDOD o como un parámetro de solicitud adicional dentro de la sección "attributes" denominado como "vmando".

Una vez que se crea una instancia de vMANDO, la información adicional representada en el descriptor conjuntamente con información de despliegue se podría incorporar, por ejemplo, en el campo "atributes" del modelo de instancia de vMANDO y definir un subcampo denominado "vmando", tal como se muestra en la Figura 5.19 (b). En la Tabla 5.12 se proporcionan detalles sobre la información adicional propuesta.

\footnotetext{
${ }^{16}$ https://developer.openstack.org/api-ref/nfv-orchestration/v1/
} 


\begin{tabular}{|l|}
\hline \multicolumn{1}{|c|}{ vmandod } \\
\hline metadata: \\
residential_network_id \\
access_network_slice_id \\
default_vnfs \\
default_sfcs \\
default_traffic_containers \\
added_vnfs \\
created_sfcs \\
created_traffic_containers \\
\hline
\end{tabular}

\begin{tabular}{|l|}
\hline request parameter \\
\hline attributes: \\
vmando: \\
residential_network_id \\
access_network_slice_id \\
default_vnfs \\
default_sfcs \\
default_traffic_containers \\
added_vnfs \\
created_sfcs \\
created_traffic_containers \\
\hline
\end{tabular}

(a)

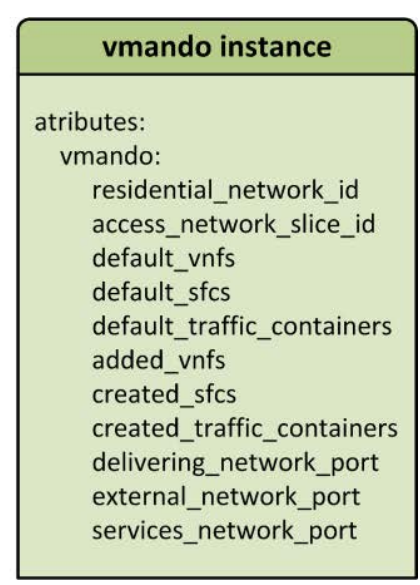

(b)

Figura 5.19 Conjunto de datos ampliado propuesto para vMANDO. (a) Datos adicionales a incluir en la sección "metadata" del descriptor o como un parámetro de solicitud. (b) Datos adicionales a incluir en el campo "attributes" de la instancia.

Tabla 5.12 Datos complementarios para definir el modelo de vMANDO.

\begin{tabular}{|c|c|c|}
\hline Datos complementarios & Tipo & Descripción \\
\hline residential_network_id & string & $\begin{array}{l}\text { Residential network's ID the vMANDO } \\
\text { belongs to }\end{array}$ \\
\hline access_network_slice_id & string & $\begin{array}{l}\text { Access network slice's ID the vMANDO } \\
\text { belongs to }\end{array}$ \\
\hline default_vnfs & $\begin{array}{l}\text { array } \\
\text { [vnf_ids] }\end{array}$ & Default VNFs of vMANDO \\
\hline default_sfcs & $\begin{array}{l}\text { array } \\
\text { [sfc_ids] }\end{array}$ & Default SFCs of vMANDO \\
\hline default_traffic_containers & $\begin{array}{l}\text { array } \\
\text { [segment_ids] }\end{array}$ & $\begin{array}{l}\text { Default traffic containers of the Residential } \\
\text { network }\end{array}$ \\
\hline added_vnfs & $\begin{array}{l}\text { array } \\
\text { [vnf_ids] }\end{array}$ & VNFs added by the user \\
\hline created_sfcs & $\begin{array}{l}\text { array } \\
\text { [sfc_ids] }\end{array}$ & SFCs created by the user \\
\hline created_traffic_containers & $\begin{array}{l}\text { array } \\
\text { [container_ids] }\end{array}$ & Traffic containers created by the user \\
\hline delivering_network_port & integer & $\begin{array}{l}\text { Delivering network's port the vMANDO is } \\
\text { connected to }\end{array}$ \\
\hline external_network_port & integer & $\begin{array}{l}\text { External network's port the vMANDO is } \\
\text { connected to }\end{array}$ \\
\hline services_network_port & integer & $\begin{array}{l}\text { Services network's port the vMANDO is } \\
\text { connected to }\end{array}$ \\
\hline
\end{tabular}

\subsubsection{TOSCA y Tacker en la Implementación del Tratamiento de Tráfico Diferenciado}

En la sección de análisis de la propuesta del capítulo 4, se puntualizó que el tratamiento de tráfico diferenciado se soporta en las interfaces MANO que controlan las operaciones del ciclo de vida de descriptores y posteriores instancias definidas por ETSI. Con base en esta afirmación y en la información 
de Tacker proporcionada en la sección 2.3.6, a continuación, se analiza si esta plataforma, conjuntamente con TOSCA, son idóneas para implementar el tratamiento de tráfico diferenciado.

Al observar la Tabla 2.1, Tabla 2.2 y Tabla 2.3, se reconoce que no se proporciona un conjunto integral y funcional del API en concordancia con las especificaciones que realiza ETSI. Por una parte, con respecto a las operaciones CRUD de descriptores, NFVO NS API y VNFFG API no permiten actualizar (método PUT) el correspondiente descriptor. Por otra parte, con respecto a las operaciones CRUD de instancias, el API en general no permite actualizarlas (método PUT). Si bien el VNFM API incluye la opción "actualizar VNF", esta opción está orientada a la actualización de los parámetros de configuración del servicio que proporciona la VNF. Esta situación representa un problema importante que limita la agilidad de la gestión de vMANDO y sus componentes considerando que al no disponer de la operación "actualizar NS o VNFFG" se tendría que forzosamente detener la instancia en ejecución, actualizar los descriptores necesarios y crear nuevamente las instancias. Este modo de trabajo resulta poco conveniente ya que implica interrumpir el servicio por un lapso de tiempo.

Como se indicó en la sección 2.3.6 del estado del arte, esta situación se replica en las demás plataformas de código abierto que implementan NFV-MANO, lo cual se debe a dos factores principales. Por una parte, como menciona Cisco [112], ETSI MANO es relativamente nuevo y proporciona solamente una descripción de alto nivel sobre cómo deberían funcionar los procesos de Gestión y Orquestación y las interfaces requeridas. Cuando se trata de operaciones del ciclo de vida utilizadas en entornos de producción, no existen especificaciones universalmente aceptadas que cumplan con los procesos específicos utilizados para incorporar, gestionar y orquestar VNFs de diferentes vendedores. Este hecho ocasiona que diferentes empresas de software y partes interesadas implementen NFV-MANO de diferentes maneras, sin consenso alguno, caso por caso y sin estándares predefinidos. Como resultado, los proveedores de servicios de red actualmente tienen varias opciones de plataformas NFV, cada una implementando una versión propia del paradigma NFV propuesto por ETSI. A esto se suma las limitadas funcionalidades de MANO que se proporcionan, las cuales son descubiertas una vez que se selecciona una plataforma y se trata de implementar un servicio en particular.

Por otra parte, la limitada implementación de las operaciones del ciclo de vida que se proporcionan, es una consecuencia del enfoque basado en plantillas de despliegue o descriptores que ETSI propone para desplegar un servicio de red. El paradigma NFV aprovecha la madurez, flexibilidad y eficiencia de las tecnologías en la Nube y, por lo tanto, trata de seguir sus principios, como por ejemplo la utilización de plantillas para desplegar aplicaciones. Chappell [44] menciona que en un entorno puro de Tecnologías de la Información (IT), donde las aplicaciones son raramente reconfiguradas una vez que han sido desplegadas, un enfoque basado en plantillas funciona adecuadamente. Si la aplicación precisa de una configuración distinta, se crea una nueva plantilla de despliegue, se elimina la aplicación con la configuración antigua y se la reemplaza mediante la creación de una nueva instancia con base en la plantilla que tiene la nueva configuración. Este enfoque no puede ser implementado en una red basada en NFV ya que las VNFs podrían requerir una configuración adicional en tiempo de ejecución, es decir, cuando la VNF haya alcanzado la etapa de disponibilidad operacional. En el caso de utilizar TOSCA como lenguaje de especificación, un operador podría requerir modificar una VNF para cumplir con nuevos requerimientos de un cliente específico, más allá de las configuraciones especificadas en la plantilla de TOSCA original. Por ejemplo, configurar nuevas reglas en el cortafuegos o agregar una nueva interfaz de red a la VNF y conectarla a una red complementaria.

A través de una plantilla de TOSCA se puede crear una instancia de un servicio de red, pero posteriormente no se puede modificar su topología bajo demanda. TOSCA por sí mismo no es un API para la modificación dinámica de instancias de servicios de red y VNFs. Esta importante desventaja del lenguaje ya ha sido reconocida por OASIS y, al tiempo que se escribe esta memoria, el comité técnico correspondiente se encuentra desarrollando un modelo de instancia ${ }^{17}$ para significativamente ampliar el soporte en la gestión continua y automatizada de topologías de TOSCA. De esta manera, se definirá el modelo de información, la semántica conductual y los métodos de acceso para una representación dinámica del modelo de objeto de los despliegues de la plantilla de servicio de TOSCA. El objetivo es proporcionar un conjunto completo de operaciones del ciclo de vida sobre las instancias de una plantilla

\footnotetext{
${ }^{17} \mathrm{https} / / /$ www.oasis-open.org/committees/tc_home.php?wg_abbrev=tosca\#openrepo
} 
de servicio, así como obtener una representación integral de su estado actual. De esta manera, sería posible observar y actuar sobre los servicios y aplicaciones desplegadas con TOSCA a medida que evolucionan con el tiempo.

Con base en el análisis realizado, la implementación de la propuesta de tratamiento de tráfico diferenciado es totalmente factible en el contexto de las operaciones del ciclo de vida e interfaces MANO propuestas por ETSI. No obstante, como ya se mencionó en la sección 4.6, la implementación real de la propuesta, así como su funcionalidad está condicionada por las capacidades útiles proporcionadas por la plataforma NFV. A esto se suma las limitaciones del propio lenguaje de especificación TOSCA en su versión actual.

En la revisión del estado del arte de la sección 2.3.4, se concluyó que la mayoría de plataformas NFV de código abierto utilizan TOSCA considerando sus destacadas características, razón por la cual fue seleccionado para especificar vMANDO y sus componentes. Si consideramos que la extensión propuesta para desarrollar un modelo de instancia para TOSCA finalmente se publique, permitiría implementar todas las funcionalidades de la propuesta de tratamiento de tráfico diferenciado. Similarmente, con base en el estado del arte, Tacker fue seleccionado como plataforma NFV de código abierto ya que utiliza TOSCA y además es un proyecto en desarrollo dentro de la comunidad de OpenStack. Desafortunadamente, las funciones de gestión y orquestación proporcionadas en la actualidad son limitadas, aunque se observa una alta actividad en el desarrollo del API.

Para solventar estos inconvenientes se podría recurrir a una plataforma NFV de pago que implemente un lenguaje de especificación distinto a TOSCA. Así, se podría utilizar por ejemplo el Orquestador de Servicios de Red (NSO) de Cisco para NFV, el cual está basado en YANG/NETCONF como lenguaje de especificación. Según consta en la descripción del producto, se proporcionan todas las operaciones del ciclo de vida de servicios de red y VNFs, tanto en descriptores como posteriores instancias de acuerdo a las recomendaciones de ETSI. Al ser una plataforma de pago, evidentemente se busca proporcionar un producto para ser utilizado en entornos de producción con el respectivo soporte. Sin embargo, este hecho compromete el principio de apertura que tanto el paradigma NFV como SDN profesan.

\subsection{Conclusiones}

El caso de uso planteado y el desarrollo de la prueba de concepto correspondiente han permitido obtener resultados prometedores que permiten validar las propuestas presentadas en esta tesis doctoral. La arquitectura de gestión propuesta, por una parte, ha permitido que se utilice el enfoque centrado en el usuario para abordar los problemas asociados a la red residencial y, por otra parte, ha facilitado el desarrollo de aplicaciones RENEMA gracias al conjunto de funciones que proporcionan los distintos RENESEs.

Para abordar los requisitos de los usuarios identificados en la sección 2.1.4, se han desarrollado dos aplicaciones RENEMA las cuales han permitido verificar la factibilidad y las ventajas de una solución de gestión basada en SDN y NFV. Por una parte, la aplicación de Control Parental ha contribuido a mejorar los aspectos de Seguridad y QoS de las redes residenciales. Con respecto a la seguridad, la definición de políticas brinda a los usuarios la capacidad de controlar fácilmente el acceso al servicio de conectividad local y externa que proporciona la red residencial. De esta manera, cualquier dispositivo desconocido por defecto tiene restringido el acceso al servicio de conectividad. Además, el establecimiento de la prioridad del servicio permite una mejor provisión de QoS ya que en la capa de infraestructura se realiza una reserva de recursos.

Por otra parte, la aplicación de Estado de la Red ha proporcionado información útil y oportuna a los usuarios. Esta aplicación, con base en la información proporcionada por NESA y NERON mostró en detalle el consumo de ancho de banda total de la red, por dispositivo, puerto y cola. Adicionalmente, con base en las notificaciones proporcionadas por el servicio de Seguimiento de Dispositivos el usuario fue alertado sobre una nueva conexión (online) o desconexión (offline) de dispositivo. De esta manera 
se ha logrado obtener una mejora notable de la Visibilidad y, por lo tanto, lo que sucede en la red residencial ya no es un misterio.

Para que las aplicaciones proporcionen la funcionalidad esperada y con el objetivo de simplificar y agilizar su implementación se ha necesitado la provisión de dos Servicios de Red Residencial, cada uno con un conjunto de funciones específicas las cuales son utilizadas a través del protocolo RENESE. Adicionalmente, NERON y NESA desempeñan un papel fundamental ya que sus funcionalidades permiten superar las limitaciones en cuanto a la provisión de QoS y monitoreo que presentan las redes residenciales de hoy en día.

La prueba de concepto desarrollada ofrece funcionalidades similares en comparación con soluciones de RGW comerciales como Linksys smart Wi-Fi ${ }^{18}$ o Netgear Genie ${ }^{19}$. Estas soluciones proporcionan un mapa de la red, control parental, medición del tráfico, priorización del tráfico y gestión remota. Sin embargo, se requiere un cierto nivel de experiencia técnica para habilitar y usar todas estas funcionalidades. Además, debido a la arquitectura cerrada de los RGW tradicionales, la adición de nuevas funcionalidades requiere una actualización de firmware y el rendimiento de las funcionalidades avanzadas puede verse afectado por los recursos computacionales limitados.

Si bien es cierto que la propuesta se basa en el uso de un RGW basado en SDN, lo cual puede considerarse como una limitación debido a la falta de productos comerciales, el enfoque centrado en el usuario y su prueba de concepto han proporcionado resultados relevantes para motivar el uso masivo de esta tecnología en las redes residenciales. Los resultados obtenidos permiten incrementar el nivel de madurez de SDN lo cual es de suma importancia para los fabricantes con el objetivo de producir RGWs compatibles con SDN comerciales.

En la parte final del capítulo se ha abordado la implementación de la propuesta de tratamiento de tráfico diferenciado para lo cual se han realizado diferentes tareas. En una primera parte, se ha definido en detalle el API REST de la aplicación de Tratamiento de Tráfico, incluyendo los parámetros de solicitud y respuesta de las operaciones CRUD de los diferentes objetos que controla la aplicación. En una segunda parte, se ha implementado vMANDO como Servicio de Red, obteniéndose el grafo de topología de conectividad de red mediante el cual se han podido obtener vMANDOD y los diferentes VNFFGDs que se presentaron en la sección 4.4.2. Estos descriptores son requeridos por la plataforma de NFV con el fin de desplegar vMANDO y todos sus componentes en la NFVI del ISP. En tercer y último lugar, se ha observado que TOSCA y Tacker, en sus versiones actuales, no son completamente idóneas para implementar el tratamiento de tráfico diferenciado. Las tareas de implementación, por una parte, han permitido validar, en cierto grado, la propuesta de tratamiento de tráfico diferenciado presentada en el capítulo 4. Por otra parte, han permitido verificar la factibilidad de implementar vMANDO y todos sus componentes en una plataforma de NFV real y, por lo tanto, demuestran las ventajas de las propuestas presentadas en comparación con el modelo de red tradicional de la red residencial. No obstante, es importante tener presente que la experimentación práctica permitiría realizar pruebas funcionales y de desempeño para complementar la validación presentada en este capítulo y obtener datos experimentales que permitan mejorar la propuesta de tratamiento de tráfico diferenciado.

\footnotetext{
${ }^{18} \mathrm{http} / / /$ www.linksys.com/us/wireless-routers/c/smart-wi-fi-wireless-routers/\#fullstory

${ }^{19} \mathrm{http}: / /$ www.netgear.com/home/discover/apps/genie.aspx
} 


\section{Capítulo 6}

\section{Conclusiones y Trabajos Futuros}

En este capítulo final, se proporciona una visión global del trabajo de investigación realizado mediante el análisis del cumplimiento de los objetivos planteados al inicio de la memoria y la obtención de conclusiones generales. Por otra parte, se muestra el alcance e impacto de la investigación al describir el proceso de divulgación de resultados el cual comprende la publicación de artículos científicos en revistas y congresos, la participación activa en proyectos nacionales y las líneas futuras de investigación que se derivan del trabajo realizado hasta el momento.

\subsection{Análisis de los Objetivos}

Como se indicó en la sección 1.2, el objetivo principal de esta tesis doctoral consistió en diseñar una nueva arquitectura de gestión para abordar tres temas puntuales de las redes residenciales: la limitada usabilidad, la alta heterogeneidad y la limitada flexibilidad para mejorar el servicio de conectividad de red provisto. Adicionalmente, se consideró proporcionar un entorno ágil y flexible para optimizar los procesos de despliegue, gestión y mantenimiento asociados a las redes residenciales. Para cumplir con este objetivo se han utilizado los paradigmas SDN y NFV conjuntamente con el enfoque centrado en el usuario. Además, se plantearon cuatro objetivos específicos, cada uno de los cuales ha sido abordado en un capítulo diferente de la tesis considerando la estrategia correspondiente previamente definida en la sección 1.2. De esta manera, una vez que se han presentado todos los capítulos, a continuación, se analiza el cumplimiento de cada objetivo:

\section{Realizar un estudio del estado del arte}

Este objetivo específico fue abordado en el segundo capítulo. Para identificar los problemas de la red residencial fue necesario comprender su origen y observar la evolución que ésta ha tenido desde su concepción hasta la actualidad. Se concluye que la red residencial fue el resultado de compartir el acceso a Internet entre diferentes dispositivos realizado por un sector de la sociedad caracterizado por tener conocimientos de redes y motivado por temas laborales. Con base en este hecho, se afirma que la red residencial ha adoptado un modelo de gestión al estilo de Internet donde se garantiza el conocimiento y la experiencia técnica. En la actualidad, este requerimiento no ha cambiado y, como consecuencia, la usabilidad de la red residencial es limitada considerando que la mayoría de usuarios no tienen conocimientos de redes. Por otra parte, esta situación también provoca que el modelo de gestión de la red no permita satisfacer la gran variedad de requerimientos de los usuarios y adaptar el servicio de conectividad provisto a los requisitos específicos de los servicios o aplicaciones que lo utilizan.

Implementar un enfoque centrado en el usuario en el modelo de gestión de la red permite solucionar en gran medida los problemas descritos previamente. No obstante, las tecnologías de redes clásicas no permiten cumplir con este objetivo de forma eficiente y no garantizan que en un futuro la solución pueda evolucionar. De esta manera, resulta imprescindible utilizar nuevos paradigmas como las Redes Definidas por Software (SDN) y la Virtualización de Funciones de Red (NFV) en la creación de una nueva arquitectura de gestión para la red residencial. 
El estado del arte muestra que existen diferentes propuestas que aprovechan los beneficios de SDN y NFV para tratar de mejorar la situación de la red residencial sin abordar de forma integral los requerimientos tanto de usuarios como de aplicaciones que hacen uso del servicio de conectividad. A esto se suma la necesidad de implementar un modelo de funcionamiento colaborativo entre los componentes SDN y NFV que forman parte de la propuesta para complementar sus aportaciones individuales y aprovechar al máximo los beneficios de estas tecnologías habilitantes.

\section{Proponer y diseñar una nueva arquitectura de gestión para la red residencial}

Este objetivo específico fue abordado en el tercer capítulo. En las conclusiones obtenidas de la revisión del estado del arte, se definieron un conjunto de requisitos que deben ser considerados en la propuesta de una solución integral para abordar los problemas presentes en la red residencial. Con base en estos requisitos se ha definido una arquitectura de gestión con base en las tecnologías SDN y NFV que permite utilizar el enfoque centrado en el usuario y facilita su implementación. Si bien el enfoque centrado en el usuario mejora la usabilidad de la red ya que permite definir herramientas y procedimientos de gestión adaptados a las habilidades y necesidades de los usuarios, es necesario abordar de igual manera el tema de la alta heterogeneidad del entorno residencial. Para este fin, las tareas de gestión se distribuyen en diferentes aplicaciones RENEMA para que el usuario sea capaz de personalizar su entorno de gestión. Con el modelo de gestión resultante, existen diferentes aplicaciones, cada una destinada a mejorar un determinado aspecto de la red como la Facilidad de Gestión, Visibilidad, QoS/QoE o Seguridad.

Por otra parte, para facilitar la implementación del enfoque centrado en el usuario, la arquitectura tiene como objetivo fomentar el desarrollo de aplicaciones RENEMA para lo cual se proporcionan diferentes RENESEs encargados de simplificar la configuración y monitoreo de red de bajo nivel. De esta manera, los desarrolladores se centran en aplicar técnicas de HCI para crear GUIs adaptadas a las habilidades de los usuarios finales y lograr la centralidad en el usuario.

Para proporcionar un modelo de gestión centrado en el usuario y basado en aplicaciones se aprovecha la programabilidad precisa del plano de datos, la comunicación de eventos de red, la alta capacidad de respuesta y el amplio conjunto de opciones para configurar políticas de QoS y monitoreo que presentan las SDNs. Además, ha sido necesario realizar una definición adicional de la arquitectura de referencia SDN para obtener el funcionamiento antes descrito. Por otra parte, se aprovechan los recursos, escalabilidad, flexibilidad y agilidad en el despliegue de servicios que proporcionan las tecnologías de la Nube conjuntamente con NFV para implementar un enfoque de gestión basado en la Nube. Este enfoque permite, en primer lugar, desplegar el sistema de gestión encargado de controlar el RGW basado en SDN y superar las restricciones en el rendimiento que las aplicaciones pudiesen experimentar debido a los recursos limitados del RGW. En segundo lugar, agregar nuevas Funciones de Red Virtualizadas (VNFs) para enriquecer el servicio de conectividad que proporciona la red residencial.

Es importante considerar que un enfoque basado en la Nube requiere el diseño específico del espacio virtual que será asignado a cada red residencial y, por lo tanto, se ha definido el Dominio de Gestión y Redes virtualizado (vMANDO). Por otra parte, al concentrar todos los componentes SDN y NFV que sustentan la red residencial en vMANDO, el ISP es capaz de alcanzar un alto grado de automatización en los procesos de despliegue, gestión y mantenimiento correspondientes.

\section{Proponer un tratamiento de tráfico diferenciado basado en SDN y NFV}

Este objetivo específico fue abordado en el cuarto capítulo. Al revisar el estado del arte, las propuestas que tratan de mejorar la situación de la red residencial implementan mayormente el enfoque vRGW soportado principalmente por NFV, excluyendo el aporte que podría proporcionar un dispositivo SDN colocado en las instalaciones del usuario. El estado del arte muestra que NFV es utilizado principalmente para externalizar funciones de red desde el RGW y desplegarlas como instancias virtuales en la NFVI del ISP. No obstante, no se aprovecha la creación de SFCs dinámicas implícita de NFV. La arquitectura de gestión propuesta va más allá del concepto de vRGW ya que no solamente permite mejorar el servicio de conectividad que ofrece la red residencial gracias a la programabilidad precisa del RGW basado en SDN y a la posibilidad de integrar nuevas VNFs al componente NFV de vMANDO, sino que proporciona un tratamiento de tráfico diferenciado para cumplir con los requisitos divergentes de los diferentes servicios o aplicaciones que se ejecutan en la red residencial. 
Para implementar un tratamiento de tráfico diferenciado, se ha utilizado el concepto de SFCs de NFV y la capacidad de SDN para direccionar un determinado tipo de tráfico a una ubicación específica. Al utilizar dos tecnologías diferentes, ha sido necesario definir nuevos puntos de referencia para que desde el sistema de gestión una aplicación RENEMA sea capaz de orquestar los componentes SDN y NFV para hacerlos trabajar de forma colaborativa y complementaria. En particular, la aplicación propuesta interactúa con las interfaces MANO y con el RGW basado en SDN.

De la propuesta de tratamiento de tráfico diferenciado también se concluye que ésta resultaría más efectiva si se integra una Red de Acceso Óptica Definida por Software (SDOAN). Este escenario proporcionaría una flexibilidad total ya que todos los actores que forman parte del entorno residencial (NFVI del ISP, red de acceso y red residencial) estarían en la capacidad de proporcionar un conjunto de interfaces que permitirían adaptar dinámicamente el servicio de conectividad de red a los requerimientos de usuarios y de los servicios o aplicaciones multimedia que se utilizan.

\section{Validar las propuestas presentadas}

Este objetivo específico fue abordado en el quinto capítulo. Considerando la estrategia definida en la sección 1.2, para validar las propuestas se ha planteado un caso de uso y se ha desarrollado la correspondiente prueba de concepto. Con el objetivo de obtener resultados fehacientes, ha sido necesario proponer y diseñar un escenario de validación compuesto por diferentes redes residenciales, la red de acceso y la NFVI del ISP. El escenario de validación considera el uso de dispositivos reales, no obstante, en una primera etapa de experimentación, se han desarrollado bancos de prueba virtual en estricto apego al escenario de validación propuesto.

Considerando que el objetivo principal de esta tesis es proponer una arquitectura de gestión, las pruebas realizadas sobre los bancos de prueba virtual están enfocadas en verificar el funcionamiento de los distintos componentes arquitectónicos en conjunto, es decir, el modelo de interacción definido para comunicar información de gestión entre las distintas capas, estados operacionales de aplicaciones RENEMA, RENESEs y Gestores, etc. Con esto en mente, se han realizado pruebas de desempeño para observar el comportamiento del retraso en la instalación de la entrada de flujo en el RGW basado en SDN lo cual indica la factibilidad para desplegar el sistema de gestión en la NFVI del ISP. Por otra parte, las pruebas de provisión de QoS han permitido observar el funcionamiento global de la arquitectura y los resultados obtenidos han permitido verificar la factibilidad de las propuestas.

En cuanto al tratamiento de tráfico diferenciado propuesto, su validación ha sido abordada desde una perspectiva de diseño. De esta manera, se ha desarrollado el proceso de implementar vMANDO como un servicio de red, obteniéndose los descriptores necesarios y analizando la factibilidad de su implementación real con TOSCA y Tacker.

\subsection{Conclusiones Generales}

En la parte final del capítulo 3 y capítulo 4 se ha realizado un análisis profundo de las propuestas considerando diferentes temas de interés con el objetivo de destacar las contribuciones, las potenciales aplicaciones y determinados aspectos que deben ser considerados para obtener un funcionamiento eficiente. Por otra parte, en cada capítulo se ha proporcionado un conjunto de conclusiones específicas que permiten identificar el problema que ha sido tratado y reconocer el trabajo realizado. A continuación, se proporciona un conjunto de conclusiones generales, con una visión más amplia del trabajo de investigación que ha sido presentado en esta tesis doctoral:

- SDN y NFV proponen un nuevo paradigma para diseñar y desplegar redes. Su promesa de permitir que la red evolucione al igual que lo hace el software y hardware ha permitido que la motivación constante dentro de la industria y de la comunidad científica sea la innovación. Las arquitecturas de referencia que proponen estas tecnologías sirven de guía y representan un punto de partida para elaborar una solución específica para un caso de uso determinado. La red residencial representa uno de los varios casos de uso que existen en la actualidad, y en la arquitectura de gestión propuesta se observa el trabajo adicional que ha sido necesario realizar para adaptar SDN y NFV al entorno residencial. De esta manera, el trabajo realizado en esta tesis y particularmente los diferentes 
conceptos definidos como el protocolo RENESE o el tratamiento de tráfico diferenciado, así como la capacidad para agrupar todos los componentes SDN y NFV que forman parte de la red residencial en vMANDO lo cual permitiría la provisión de la Red Residencial como Servicio (RNaaS), representan contribuciones valiosas que pueden ser consideradas por la ONF o ETSI para perfeccionar y avanzar en la definición de los estándares de las arquitecturas SDN y NFV. Adicionalmente, el trabajo realizado en esta tesis al proporcionar evidencias sólidas de los beneficios de SDN y NFV, incrementa el grado de madurez de estas tecnologías y motiva su adopción en entornos distintos en los cuales usualmente se utilizan como son los Centros de Datos, por ejemplo.

- El concepto de Servicios de Red Residencial (RENESEs) al crear un nivel de abstracción mayor sobre los servicios básicos que proporciona el controlador SDN para ocultar los detalles de configuración y monitoreo de red de bajo nivel está relacionado con el concepto de interfaz Northbound basada en "intents" propuesta por la ONF (sección 2.2.3). De acuerdo con el paradigma basado en "intents", el conjunto de RENESEs representa el sistema Proveedor mientras que las diferentes aplicaciones representan el sistema Consumidor. De esta manera, las aplicaciones saben el "qué" y el "por qué" del servicio y lo utilizan a través del conjunto de APIs implementado mientras que los RENESEs determinan "cómo" proporcionar el servicio.

El servicio de conmutación podría ser considerado un ejemplo de un API basado en "intents" ya que abstrae todo el servicio que se encarga de realizar la conmutación de paquetes en el RGW basado en SDN y lo muestra como un servicio de conectividad. Incluye la parte declarativa, que permite que la aplicación específica qué hacer, en este caso, proporcionar conectividad a un determinado dispositivo con base en una política de navegación específica, en lugar de como hacerlo, ya que el propio servicio se encarga de obtener las reglas OpenFlow y enviarlas al RGW basado en SDN. Es importante mencionar que al crear un nivel más de abstracción, el sistema proveedor se encarga de automatizar procesos y encapsularlos como servicios para ponerlos a disposición de sistemas consumidores con el objetivo de no replicar esfuerzos y simplificar y agilizar el desarrollo de servicios más avanzados a través de la composición.

Resulta de gran interés también considerar el uso de Northbound interfaces basadas en "intents" para abordar el tema de la interoperabilidad/portabilidad de aplicaciones SDN. Al proporcionar una interfaz con un alto nivel de abstracción se podrían implementar "intents" estándar que deberían ser proporcionados por la mayoría de controladores, eliminando de esta manera la dependencia de una tecnología de controlador en particular.

- El tratamiento de tráfico diferenciado con soporte de QoS propuesto, puede ser considerado como un caso de uso del funcionamiento complementario de SDN y NFV, el cual representa un tema de discusión actual en el estado del arte y que plantea una serie de desafíos para conseguirlo. En esta tesis, se ha propuesto un mecanismo que permite complementar las aportaciones de SDN y NFV mediante la orquestación de la plataforma NFV, el RGW basado en SDN y la SDOAN que realiza el sistema de gestión centrado en el usuario. La orquestación la realiza una aplicación RENEMA y consiste en utilizar, por una parte, las interfaces NFV-MANO para crear SFCs encargadas de aplicar un tratamiento en particular y, por otra parte, los Servicios de Red Residencial (RENESEs) que proporciona la capa de aplicación SDN para diferenciar el tráfico y direccionarlo a la SFC adecuada.

Si bien en el capítulo se ha puesto énfasis en la interacción con MANO y el RGW basado en SDN, la propuesta considera que las características funcionales de la SDOAN también pueden ser controlables por una aplicación RENEMA. Para hacer partícipe a la red de acceso del tratamiento de tráfico diferenciado, sería necesario definir un bloque funcional en la aplicación encargada de implementarlo. Este bloque funcional se encargaría de utilizar las interfaces que proporciona el sistema de control de la SDOAN para configurar las características funcionales de acuerdo con los requerimientos del usuario.

- Al hablar de redes residenciales debemos notar que varios ambientes como oficinas pequeñas, comercios pequeños, hoteles, comunidad de vecinos, residencia de estudiantes, entre otros, exhiben características similares y, por lo tanto, se podrían beneficiar de las propuestas desarrolladas para las redes residenciales. Resultaría interesante, por ejemplo, mediante el sistema de gestión propuesto 
definir políticas de navegación en Internet para cada huésped de un hotel con base en sus requerimientos específicos y de esta manera optimizar el servicio provisto.

Por otra parte, el sistema de gestión propuesto también podría ser implementado en entornos gestionados donde se garantiza la experiencia y conocimientos técnicos considerando que el desarrollo de las aplicaciones debería estar orientado hacia el personal técnico que las va a utilizar. De esta manera se podría configurar un entorno de gestión que responda a las necesidades y objetivos de la empresa o institución de tal manera que se pueda simplificar y optimizar los procesos operativos y de gestión de la red.

\subsection{Divulgación de Resultados}

El trabajo de investigación realizado en esta tesis doctoral ha permitido contribuir con el estado del arte mediante publicaciones en revistas con factor de impacto (JCR) y conferencias, así como al desarrollo de proyectos españoles. A continuación, se muestran las publicaciones realizadas y se describen los proyectos relacionados.

\subsubsection{Publicaciones}

Las propuestas presentadas en esta tesis doctoral se resumen en las siguientes publicaciones:

\section{- Revistas}

- R. Flores Moyano, D. Fernández Cambronero, and L. Bellido Triana, "A user-centric SDN management architecture for NFV-based residential networks," Computer Standards \& Interfaces, vol. 54, pp. 279-292, 2017.

Cuartil JCR: Q2 (2016)

Factor de Impacto: 1.633

- R. Flores Moyano, D. Fernández, L. Bellido, and C. González, "A software-defined networking approach to improve service provision in residential networks," International Journal of Network Management, vol. 27, no. 6, 2017.

Cuartil JCR: Q3 (2016)

Factor de Impacto: 1.118

\section{- Conferencia}

- R. Flores Moyano, D. Fernández, L. Bellido, N. Merayo, J. C. Aguado, and I. de Miguel, "NFVbased QoS provision for Software Defined Optical Access and residential networks," in Quality of Service (IWQoS), 2017 IEEE/ACM 25th International Symposium on, 2017, pp. 1-5.

Lugar y fecha de conferencia: Vilanova i la Geltru, España, 14-16 de junio de 2017

Ranking CORE: B (2018)

Ranking GII-GRIN-SCIE (GGS): B (2017)

\section{- Workshops}

- R. Flores Moyano, D. Fernández, L. Bellido, C. Lentisco, E. Pastor, J. Ruiz and O. Walid, "A User-Centric cloud-based SDN/NFV architecture for Residential Networks," in 1st International Workshop on Elastic Networks Design and Optimisation (ELASTICNETS 2016), 2016, pp. 1-5.

Lugar y fecha de conferencia: Cartagena, España, 12 y 13 de mayo de 2016

- El artículo titulado "NFV-based QoS provision for Software Defined Optical Access and residential networks" presentado en $I W Q o S 2017$ también fue presentado en 2nd International Workshop on Elastic Networks Design and Optimisation (ELASTICNETS 2017).

Lugar y fecha del workshop: Vilanova i la Geltru, España, 13-14 de junio de 2017 


\subsubsection{Proyectos Nacionales}

Las propuestas presentadas en esta tesis doctoral están relacionadas con tres proyectos nacionales los cuales se describen a continuación:

\section{- Proyecto INNPACTO CloudTrust}

Este proyecto fue financiado por el Ministerio de Ciencia e Innovación de España (contrato ${ }^{\circ}$ IPT2012-0876-390000). Estuvo integrado por las empresas Innovati, Alcatel-Lucent y el grupo de investigación GIROS de la ETSIT-UPM. El proyecto inició en enero de 2013 y se extendió hasta el primer trimestre de 2015. El proyecto permitió adquirir experiencia en el uso de las tecnologías SDN y NFV lo cual resultó de gran importancia para definir una línea de investigación en la cual se centra la presente tesis doctoral, así como esbozar las primeras ideas.

El objetivo general del proyecto Cloudtrust se centró en el estudio y experimentación con escenarios de servicios de telecomunicaciones ofrecidos a usuarios residenciales en los que se virtualizan las funciones típicas del equipamiento de red del usuario (Customer Premises Equipment o abreviadamente $\mathrm{CPE}$ ). Se planteó sustituir el CPE por un equipo de red más sencillo y eficiente que se ocupe únicamente de las funciones de conmutación del tráfico, moviendo la inteligencia de los servicios hacia servidores en la Nube utilizando las tecnologías SDN y NFV.

\section{- Proyecto GREDOS}

Este proyecto es financiado por el Ministerio de Economía y Competitividad de España (contrato ${ }^{\circ}$ TEC2015-67834-R). Es desarrollado por el grupo de investigación GIROS de la ETSIT-UPM. El proyecto inició en enero de 2016 y tiene una duración de tres años. Las ideas presentadas en esta tesis doctoral han sido de gran utilidad en la propuesta y posterior desarrollo del proyecto GREDOS.

El proyecto GREDOS plantea cuatro líneas de trabajo. La primera de ellas busca realizar propuestas para nuevas arquitecturas de red basadas en SDN para los entornos residencial y de campus, y sobre esas arquitecturas contribuirá a la formalización del modelo de interacción entre aplicación de red y controlador mediante la definición de interfaces estándar. La segunda línea de trabajo abordará la provisión de calidad de servicio extremo a extremo, centrándose en la coordinación mediante SDN de los dispositivos de red y las redes de distribución de contenidos. La tercera línea de trabajo buscará mejorar mediante SDN la gestión de los múltiples dispositivos y servicios que conforman la Internet de las cosas en los ámbitos de las redes residenciales y de campus. Finalmente, la cuarta línea se centrará en el desarrollo e integración de herramientas de creación de testbeds virtuales para SDN. El proyecto contempla, además, la creación de una plataforma de experimentación basada en la combinación de escenarios virtuales y equipos reales que permita validar las arquitecturas y modelos planteados utilizando las herramientas desarrolladas.

\section{- Proyecto ElasticNetworks}

Este proyecto es financiado por el Gobierno de España (contrato $n^{\circ}$ TEC2015-71932-REDT). El proyecto inició en noviembre de 2015 y se extenderá hasta noviembre de 2018. Representa una red temática compuesta por grupos de investigación pertenecientes a diferentes universidades de España. En los workshops organizados por la red temática, se ha presentado el trabajo de investigación realizado. Además, se ha establecido una colaboración con el Grupo de Comunicaciones Ópticas de la Universidad de Valladolid de la cual se ha obtenido un artículo presentado en el IWQoS 2017.

El objetivo de ElasticNetworks es cumplir con el desafío tecnológico de diseñar una red que permita basar la vida del ciudadano en el Fog y Cloud Computing. En particular, ElasticNetworks contribuirá a un rediseño holístico de la red de transporte hacia una red elástica más ágil, flexible y programable, pero con un bajo costo de operación, implementación y mantenimiento, y de bajo consumo de energía. Esta será una red superior con respecto a la actual en términos de rendimiento, capaz de aprovisionar la capacidad de red cuando sea necesario, con una red dinámica y móvil con granularidad de gran ancho de banda, capaz de hacer frente al advenimiento del mundo inteligente totalmente conectado. 


\subsection{Trabajos Futuros}

Ciertamente, la prueba de concepto y los diferentes escenarios virtuales de validación han proporcionado resultados positivos que demuestran el valor y la importancia de la propuesta de una nueva arquitectura de gestión para la red residencial. No obstante, luego de haber realizado diferentes experimentos y análisis es importante reconocer que existen diferentes temas que necesitan un trabajo adicional ya que, al tratarse de un tema complejo, no todos han podido tratarse con detalle. De esta manera, estos temas pendientes, representan futuras líneas de investigación que se explican a continuación:

- La Red Residencial como Servicio (RNaaS) representa un concepto innovador que resulta de la unión de las diferentes propuestas que se presentan en esta tesis y, por lo tanto, representa el escenario objetivo al cual conduce el trabajo realizado. En este sentido, es importante finalizar la implementación del escenario de validación tanto en la parte física compuesta por la red residencial, la red de acceso y la NFVI del ISP, así como en la parte de gestión y control compuesta por NFVMANO, el sistema de gestión centrado en el usuario y el control de la SDOAN.

En lo que respecta a NFV, es importante considerar una plataforma que proporcione un conjunto de interfaces MANO que realmente permita controlar las operaciones CRUD de Servicios de Red, VNFs, SFCs, etc. y que mejor se ajuste a los requisitos de vMANDO. En esta tesis se ha utilizado Tacker, no obstante, dadas sus limitadas opciones, se recomienda utilizar otra plataforma de las que se mencionan en el capítulo de estado del arte como ONAP, OSM u OpenBaton.

En lo que respecta a la red de acceso, el proceso de integración debe abordarse casi en su totalidad. Si bien en la sección 4.2 se realiza una descripción general de la propuesta, es necesario profundizar en la interacción entre el sistema de gestión centrado en el usuario y el control de la SDOAN. De esta manera, por una parte, se deben definir los procedimientos y semántica para intercambiar información de gestión y, por otra parte, el bloque funcional de la aplicación RENEMA encargado de realizar tales procedimientos. Este enfoque considera a la SDOAN (plano de datos y control) como una entidad independiente y, por lo tanto, el sistema de gestión debe interactuar con ésta a través de un determinado punto de referencia. No obstante, como se indicó en la sección 4.2.2, existe la posibilidad de que la plataforma de NFV proporcione de forma nativa las porciones virtuales de red de acceso. Considerando el escenario de validación propuesto en la sección 5.3.2, se podría realizar el proceso de integrar la OLT como hardware de red en la NFVI y desarrollar las interfaces de control para incluirlas en MANO. En este escenario, la SDOAN forma parte de la plataforma de NFV y se proporciona un conjunto de interfaces para controlar la virtualización y despliegue de la red de acceso. Con este enfoque, el sistema de gestión interactuaría solamente con MANO a través de un determinado punto de referencia.

El proceso de integración de la red de acceso permitiría contribuir a avanzar en la provisión de redes de acceso virtual y soportar el concepto de la Red Residencial como Servicio. Adicionalmente, una vez que se cuente con un prototipo totalmente funcional, se podrán realizar pruebas exhaustivas para evaluar la factibilidad de las propuestas en un escenario global, así como perfeccionarlas.

- En esta tesis se menciona la importancia de implementar un enfoque interdisciplinario para lograr la centralidad en el usuario. En este contexto, la arquitectura de gestión propuesta representa la contribución que realiza la disciplina de redes, es decir, la arquitectura proporciona el entorno adecuado para que las aplicaciones RENEMA puedan ejecutarse. La arquitectura en sí representa un medio para conseguir la centralidad en el usuario. De esta manera, es necesario complementar el enfoque al incluir técnicas de interacción hombre-computadora (HCI) que permitan crear aplicaciones adaptadas a las habilidades y necesidades de los usuarios.

Una vez que se obtengan aplicaciones RENEMA desarrolladas de forma profesional, resulta imprescindible definir metodologías para realizar experimentos con usuarios en entornos residenciales reales que permitan medir en que porcentaje mejora la usabilidad de la red y que tan efectivo resulta ser el enfoque centrado en el usuario para abordar el tema de la alta heterogeneidad del entorno residencial. 
- Al mover el plano de control SDN (sistema de gestión) a las instalaciones del ISP con el objetivo de implementar un enfoque de gestión basado en la Nube y considerando que se utiliza un control en banda, es indispensable garantizar que la conectividad entre la red residencial y la NFVI del ISP se mantenga siempre activa. Una eventual pérdida de conectividad provocaría que el RGW basado en SDN no pueda comunicarse con su plano de control y, por lo tanto, el usuario sería incapaz de gestionar la red residencial. El estado del arte indica que la pérdida de conectividad entre el plano de datos y control es uno de los posibles fallos a los cuales debe enfrentarse el paradigma SDN. A esto se suma los fallos asociados al plano de datos y al plano de control.

En este contexto, una solución basada en SDN tolerante a fallos debería proporcionar replicas o respaldos de los componentes. En el caso de la red residencial, se podría colocar un controlador secundario dentro del RGW basado en SDN que implemente una versión simplificada del sistema de gestión a cargo de mantener las comunicaciones locales o dirigir el tráfico por un enlace de respaldo si existiese. Esta solución permitiría abordar los fallos relativos al canal de comunicación y al plano de control. Proveer a la red residencial de un enlace de respaldo representa una opción más compleja desde el punto de vista técnico y el gasto económico asociado puede resultar excesivo. Además, considerando que la NFVI del ISP podría experimentar eventuales fallos, es necesario que el ISP implemente réplicas del sistema de gestión para evitar interrupciones del servicio mediante controladores multicapa, controladores distribuidos, configuración maestro-esclavo, entre otras. En definitiva, es necesario estudiar más en detalle los fallos asociados a SDN para proporcionar una solución adecuada y efectiva que se adapte a las características del entorno residencial.

- Como se mencionó anteriormente, la arquitectura propuesta permite utilizar un enfoque centrado en el usuario y facilita su implementación. De esta manera, considerando la complejidad en cuanto al diseño, desarrollo y posterior optimización que pueden presentar los diferentes bloques funcionales que forman parte de la arquitectura, estos representan por sí mismos líneas futuras de investigación. Por ejemplo, la arquitectura incluye los bloques funcionales NERON para proporcionar QoS y NESA para proporcionar estadísticas, pero solamente se han definido un conjunto de funciones mínimas a ser provistas. Así, es necesario realizar un análisis adicional de NERON y NESA teniendo en cuenta las características específicas del tráfico de la red residencial y de la red del ISP.

Al mencionar la complejidad que pueden presentar los diferentes bloques funcionales de la arquitectura, resulta necesario de igual manera abordar tres aspectos puntuales. En primer lugar, la seguridad del entorno de gestión ya que la red residencial se gestionará desde diferentes dispositivos (smartphones o tablets, por ejemplo) o incluso por diferentes usuarios (Alice o Bob, por ejemplo). De esta manera, es necesario implementar mecanismos de autenticación y autorización para restringir el acceso a usuarios no autorizados o que aplicaciones peligrosas se incorporen al sistema y comprometan la integridad del entorno. Por otra parte, considerando que el dominio lógico de la red residencial se extiende hasta las instalaciones del ISP debido a que se desplaza la función de gateway, es necesario que se implementen medidas de seguridad adicionales en vMANDO destinadas a evitar conexiones no deseadas que de igual manera comprometan la red residencial y obtengan información confidencial que ésta transporta.

En segundo lugar, la implementación de la tienda de aplicaciones RENEMA. Este aspecto resulta complejo ya que es necesario definir en detalle los procesos relacionados a las aplicaciones como la instalación, desinstalación, actualización, asignación de recursos, etc. A esto se suma la posible definición de bloques funcionales complementarios que soporten las tareas definidas para el Gestor de aplicaciones. De esta manera, resultaría de gran interés incluir la tienda de aplicaciones en las pruebas a ser realizadas con el prototipo final.

En tercer lugar, aunque la provisión de un canal dedicado para el tráfico de gestión en banda implementado parece ser una solución adecuada para evitar la congestión de la conexión a Internet de la red residencial, es necesario determinar analíticamente el ancho de banda adecuado a ser asignado. Para este fin, se podría definir una metodología para caracterizar el tráfico de gestión y establecer un intervalo de valores de ancho de banda que garanticen la comunicación correcta entre el RGW basado en SDN y el sistema de gestión. 


\section{Referencias}

1. Broadband Commission, "The State of Broadband 2017: Broadband Catalyzing Sustainable Development," United Nations, Geneva, Switzerland, 2017. [Online]. Available: https://www.itu.int/dms_pub/itu-s/opb/pol/S-POL-BROADBAND.18-2017-PDF-E.pdf. [Accessed: 21-Dec-2017].

2. R. E. Grinter, W. K. Edwards, M. W. Newman, and N. Ducheneaut, "The work to make a home network work," in ECSCW 2005, 2005, pp. 469-488.

3. W. K. Edwards, R. E. Grinter, R. Mahajan, and D. Wetherall, "Advancing the state of home networking," Commun. ACM, vol. 54, no. 6, pp. 62-71, 2011.

4. $\quad$ R. E. Grinter et al., "The ins and outs of home networking: The case for useful and usable domestic networking," ACM Trans. Comput. Interact., vol. 16, no. 2, p. 8, 2009.

5. J. N. Cummings and R. Kraut, "Domesticating computers and the Internet," Inf. Soc., vol. 18, no. 3, pp. 221-231, 2002.

6. S. N. Parekh, "Evolution of wireless home networks: The role of policy-Makers in a standardsbased market," Massachusetts Institute of Technology, Technology and Policy Program, 2001.

7. ITU-T, "Recommendation H.622, A generic home network architecture with support for multimedia services," 2008. [Online]. Available: https://www.itu.int/rec/T-REC-H.622-200806I/en. [Accessed: 13-Sep-2017].

8. Broadband Forum, "TR-094: Multi-Service Delivery Framework for Home Networks," 2004. [Online]. Available: https://www.broadband-forum.org/technical/download/TR-094.pdf. [Accessed: 18-Sep-2017].

9. Internet Engineering Task Force (IETF), "Network Configuration Protocol (NETCONF) RFC 6241," 2011. [Online]. Available: https://tools.ietf.org/pdf/rfc6241.pdf. [Accessed: 20-Sep2017].

10. Broadband Forum, "TR-069 CPE WAN Management Protocol," 2013. [Online]. Available: https://www.broadband-forum.org/technical/download/TR-069_Amendment-5.pdf. [Accessed: 20-Sep-2017].

11. Home Gateway Initiative, "Home Gateway Technical Requirements: Residential profile v1.0 (HGI-RD001-R2.01)," $2008 . \quad$ [Online]. Available: http://www.homegatewayinitiative.org/userfiles/file/downloads/RD-001-R2-01_HG-Tech-ReqResidential_V1-01.pdf. [Accessed: 20-Sep-2017].

12. ITU, "Recommendation X.700, Management framework for Open Systems Interconnection (OSI) for CCITT applications," 1992. [Online]. Available: https://www.itu.int/rec/dologin_pub.asp?lang=e\&id=T-REC-X.700-199209-I!!PDFE\&type=items. [Accessed: 21-Sep-2017].

13. E. Shehan and W. K. Edwards, "Home networking and HCI: what hath god wrought?," in Proceedings of the SIGCHI conference on Human factors in computing systems, 2007, pp. 547556.

14. M. Chetty, D. Haslem, A. Baird, U. Ofoha, B. Sumner, and R. Grinter, "Why is my internet slow?: making network speeds visible," in Proceedings of the SIGCHI Conference on Human Factors in Computing Systems, 2011, pp. 1889-1898.

15. G. Liu, S. Zhou, X. Zhou, and X. Huang, "QoS management in home network," in Computational Intelligence for Modelling, Control and Automation, 2006 and International 
Conference on Intelligent Agents, Web Technologies and Internet Commerce, International Conference on, 2006, p. 203.

16. Home Gateway Initiative, "QoS white paper (HGI-GD013-R2)," 2009. [Online]. Available: http://www.homegatewayinitiative.org/userfiles/file/downloads/HGI-GD013-R2.pdf. [Accessed: 10-Oct-2017].

17. T. Stimpson, L. Liu, J. Zhang, R. Hill, W. Liu, and Y. Zhan, "Assessment of security and vulnerability of home wireless networks," in Fuzzy Systems and Knowledge Discovery (FSKD), 2012 9th International Conference on, 2012, pp. 2133-2137.

18. J. B. Gross and M. B. Rosson, "Looking for trouble: understanding end-user security management," in Proceedings of the 2007 Symposium on Computer Human interaction For the Management of information Technology, 2007, p. 10.

19. B. Nunes, M. Mendonca, X.-N. Nguyen, K. Obraczka, and T. Turletti, "A survey of softwaredefined networking: Past, present, and future of programmable networks," Commun. Surv. Tutorials, IEEE, vol. 16, no. 3, pp. 1617-1634, 2014.

20. N. Feamster, J. Rexford, and E. Zegura, "The road to SDN: an intellectual history of programmable networks," ACM SIGCOMM Comput. Commun. Rev., vol. 44, no. 2, pp. 87-98, 2014.

21. Open Networking Foundation, "Software Defined Networking: The new norm for networks," 2012. [Online]. Available: https://www.opennetworking.org/sdnresources/whitepapers/software-defined-networking-the-new-norm-for-networks/. [Accessed: 26-Sep-2017].

22. Open Networking Foundation, "SDN Architecture Issue 1 (ONF TR-502)," 2014. [Online]. Available: $\quad$ https://www.opennetworking.org/wpcontent/uploads/2013/02/TR_SDN_ARCH_1.0_06062014.pdf. [Accessed: 27-Sep-2017].

23. V. Shukla, Introduction to Software Defined Networking: Openflow \& VxLAN. CreateSpace Independent Publishing Platform, 2013.

24. P. Goransson, C. Black, and T. Culver, Software defined networks: a comprehensive approach (Second Edition). Morgan Kaufmann, 2017.

25. P. A. Morreale and J. M. Anderson, Software Defined Networking: Design and Deployment. CRC Press, 2015.

26. C. Trois, M. D. Del Fabro, L. C. E. de Bona, and M. Martinello, "A survey on SDN programming languages: toward a taxonomy," IEEE Commun. Surv. Tutorials, vol. 18, no. 4, pp. 2687-2712, 2016.

27. Open Networking Foundation, "Real Time Media NBI REST specification (ONF TR-517)," 2015. [Online]. Available: https://3vf60mmveq1g8vzn48q2o71a-wpengine.netdna-ssl.com/wpcontent/uploads/2014/10/Real_Time_Media_NBI_REST_Specification.pdf. [Accessed: 03-Oct2017].

28. Software-Defined Networking Research Group (SDNRG), "Requirements and Design Patterns for REST Northbound API in SDN," 2016. [Online]. Available: https://tools.ietf.org/pdf/draftli-sdnrg-design-restapi-02.pdf. [Accessed: 03-Oct-2017].

29. L. Li, W. Chou, W. Zhou, and M. Luo, "Design Patterns and Extensibility of REST API for Networking Applications," IEEE Trans. Netw. Serv. Manag., vol. 13, no. 1, pp. 154-167, 2016.

30. Open Networking Foundation, "Intent NBI - Definition and Principles (ONF TR-523)," 2016. [Online]. Available: https://3vf60mmveq1g8vzn48q2o71a-wpengine.netdna-ssl.com/wpcontent/uploads/2014/10/TR-523_Intent_Definition_Principles.pdf. [Accessed: 03-Oct-2017].

31. R. Chayapathi, S. F. Hassan, and Paresh Shah, Network Function Virtualization (NFV) with a Touch of SDN. Addison Wesley, 2017. 
32. D. Kreutz, F. M. V Ramos, P. E. Verissimo, C. E. Rothenberg, S. Azodolmolky, and S. Uhlig, "Software-defined networking: A comprehensive survey," Proc. IEEE, vol. 103, no. 1, pp. 14$76,2015$.

33. O. Salman, I. H. Elhajj, A. Kayssi, and A. Chehab, "SDN controllers: A comparative study," in Electrotechnical Conference (MELECON), 2016 18th Mediterranean, 2016, pp. 1-6.

34. J. H. Cox et al., "Advancing Software-Defined Networks: A Survey," IEEE Access, 2017.

35. A. Bondkovskii, J. Keeney, S. van der Meer, and S. Weber, "Qualitative comparison of opensource SDN controllers," in Network Operations and Management Symposium (NOMS), 2016 IEEE/IFIP, 2016, pp. 889-894.

36. R. Mijumbi, J. Serrat, J.-L. Gorricho, N. Bouten, F. De Turck, and R. Boutaba, "Network function virtualization: State-of-the-art and research challenges," IEEE Commun. Surv. Tutorials, vol. 18, no. 1, pp. 236-262, 2016.

37. M. Chiosi et al., "Network functions virtualisation: An introduction, benefits, enablers, challenges and call for action," in SDN and OpenFlow World Congress, 2012, pp. 22-24.

38. W. Stallings, Foundations of modern networking: SDN, NFV, QoE, IoT, and Cloud. AddisonWesley Professional, 2015.

39. ETSI, "Network Functions Virtualisation (NFV); Architectural Framework (v1.2.1)," 2014. [Online].

Available: http://www.etsi.org/deliver/etsi_gs/NFV/001_099/002/01.02.01_60/gs_NFV002v010201p.pdf. [Accessed: 06-Oct-2017].

40. OASIS Committee Specification Draft 03, "TOSCA Simple Profile for Network Functions Virtualization (NFV) Version 1.0," 2016. [Online]. Available: http://docs.oasisopen.org/tosca/tosca-nfv/v1.0/csd03/tosca-nfv-v1.0-csd03.html. [Accessed: 08-Mar-2018].

41. Internet Engineering Task Force (IETF), "Service Function Chaining (SFC) Architecture RFC 7665," 2015. [Online]. Available: https://www.rfc-editor.org/rfc/pdfrfc/rfc7665.txt.pdf. [Accessed: 12-Oct-2017].

42. ETSI, "Network Function Virtualisation (NFV); Management and Orchestration v1.1.1," 2014. [Online]. Available: http://www.etsi.org/deliver/etsi_gs/NFVMAN/001_099/001/01.01.01_60/gs_NFV-MAN001v010101p.pdf. [Accessed: 08-Mar-2018].

43. J. Garay, J. Matias, J. Unzilla, and E. Jacob, "Service description in the NFV revolution: Trends, challenges and a way forward," IEEE Commun. Mag., vol. 54, no. 3, pp. 68-74, 2016.

44. C. Chappell, "Deploying virtual network functions: The complementary roles of TOSCA and NETCONF/YANG," White Paper, 2015. [Online]. Available: http://www.tailf.com/wordpress/wp-content/uploads/2015/02/HR-Cisco-ALU-TOSCA-YANG-WP-2-1715.pdf. [Accessed: 28-Jul-2017].

45. B. Di Martino, G. Cretella, and A. Esposito, "Defining cloud services workflow: a comparison between TOSCA and OpenStack hot," in Complex, Intelligent, and Software Intensive Systems (CISIS), 2015 Ninth International Conference on, 2015, pp. 541-546.

46. Internet Engineering Task Force (IETF), "YANG - A Data Modeling Language for the Network Configuration Protocol (NETCONF) RFC 6020," 2010. [Online]. Available: https://tools.ietf.org/pdf/rfc6020.pdf. [Accessed: 17-Oct-2017].

47. Distribute Management Task Force (DMTF), "Open Virtualization Format Specification (DSP0243)," 2015. [Online]. Available: https://www.dmtf.org/sites/default/files/standards/documents/DSP0243_2.1.1.pdf. [Accessed: 17-Oct-2017].

48. P. Lipton, "Escaping vendor lock-in with tosca, an emerging cloud standard for portability," $C A$ Labs Res., vol. 49, 2012. 
49. R. Mijumbi, J. Serrat, J.-L. Gorricho, S. Latré, M. Charalambides, and D. Lopez, "Management and Orchestration Challenges in Network Functions Virtualization," IEEE Commun. Mag., vol. 54, no. 1, pp. 98-105, 2016.

50. J. de J. G. Herrera and J. F. B. Vega, "Network functions virtualization: A survey," IEEE Lat. Am. Trans., vol. 14, no. 2, pp. 983-997, 2016.

51. SDx Central, "2017 NFV Report Series Part 2: Orchestrating NFV - MANO and Service Assurance," 2017. [Online]. Available: https://www.sdxcentral.com/reports/nfv-mano-andservice-assurance-download-2017/. [Accessed: 23-Oct-2017].

52. M.-A. Kourtis et al., "T-NOVA: An Open-Source MANO Stack for NFV Infrastructures," IEEE Trans. Netw. Serv. Manag., vol. 14, no. 3, pp. 586-602, 2017.

53. M. Ibáñez, N. M. Madrid, and R. Seepold, "Virtualization of residential gateways," in Intelligent Solutions in Embedded Systems, 2007 Fifth Workshop on, 2007, pp. 115-125.

54. J. Whiteaker et al., "Expanding home services with advanced gateways," ACM SIGCOMM Comput. Commun. Rev., vol. 42, no. 5, pp. 37-43, 2012.

55. T. Cruz, P. Simões, N. Reis, E. Monteiro, F. Bastos, and A. Laranjeira, "Cruz, T. et al. (2013) 'An architecture for virtualized home gateways', in Integrated Network Management (IM 2013), 2013 IFIP/IEEE International Symposium on. IEEE, pp. 520-526.An architecture for virtualized home gateways," in Integrated Network Management (IM 2013), 2013 IFIP/IEEE International Symposium on, 2013, pp. 520-526.

56. ETSI, "Network Functions Virtualisation (NFV); Use Cases (V1.1.1)," 2013. [Online]. Available:

http://www.etsi.org/deliver/etsi_gs/nfv/001_099/001/01.01.01_60/gs_nfv001v010101p.pdf. [Accessed: 12-Oct-2017].

57. Broadband Forum, "TR-317 Network Enhanced Residential Gateway," 2016. [Online]. Available: https://www.broadband-forum.org/technical/download/TR-317.pdf. [Accessed: 12Oct-2017].

58. Broadband Forum, "TR-345 Broadband Network Gateway and Network Function Virtualization," 2016. [Online]. Available: https://www.broadbandforum.org/technical/download/TR-345.pdf. [Accessed: 12-Oct-2017].

59. OPNFV \& OpenDayLight, "Virtualizing Customer Premises with Service Function Chaining," 2016. [Online]. Available: https://www.opnfv.org/wpcontent/uploads/sites/12/2016/11/opnfv_odl_vcpe_sfc_brief.pdf. [Accessed: 30-Oct-2017].

60. SDx Central, "2017 SD-WAN and Virtual Edge Report The Evolving SD-WAN, vCPE and uCPE Landscape," 2017. [Online]. Available: https://www.sdxcentral.com/reports/2017/sdwan-vcpe-ucpe/. [Accessed: 30-Oct-2017].

61. L. Peterson et al., "Central office re-architected as a data center," IEEE Commun. Mag., vol. 54, no. 10, pp. 96-101, 2016.

62. Y. Yiakoumis, S. Katti, T.-Y. Huang, N. McKeown, K.-K. Yap, and R. Johari, "Putting home users in charge of their network," in Proceedings of the 2012 ACM Conference on Ubiquitous Computing, 2012, pp. 1114-1119.

63. H. H. Gharakheili, J. Bass, L. Exton, and V. Sivaraman, "Personalizing the home network experience using cloud-based SDN," in A World of Wireless, Mobile and Multimedia Networks (WoWMoM), 2014 IEEE 15th International Symposium on, 2014, pp. 1-6.

64. D. Pediaditakis, A. Gopalan, N. Dulay, M. Sloman, and T. Lodge, "Home network management policies: Putting the user in the loop," in Policies for Distributed Systems and Networks (POLICY), 2012 IEEE International Symposium on, 2012, pp. 9-16.

65. R. Mortier et al., "Control and understanding: Owning your home network," in Communication 
Systems and Networks (COMSNETS), 2012 Fourth International Conference on, 2012, pp. 110.

66. M. Boussard et al., "Software-Defined LANs for Interconnected Smart Environment," in Teletraffic Congress (ITC 27), 2015 27th International, 2015, pp. 219-227.

67. H. H. Gharakheili, L. Exton, V. Sivaraman, J. Matthews, and C. Russell, "Third-party customization of residential Internet sharing using SDN," in Telecommunication Networks and Applications Conference (ITNAC), 2015 International, 2015, pp. 214-219.

68. H. Kim and N. Feamster, "Improving network management with software defined networking," Commun. Mag. IEEE, vol. 51, no. 2, pp. 114-119, 2013.

69. K. L. Calvert, W. K. Edwards, N. Feamster, R. E. Grinter, Y. Deng, and X. Zhou, "Instrumenting home networks," ACM SIGCOMM Comput. Commun. Rev., vol. 41, no. 1, pp. 84-89, 2011.

70. N. Feamster, "Outsourcing home network security," in Proceedings of the 2010 ACM SIGCOMM workshop on Home networks, 2010, pp. 37-42.

71. S. Luo, J. Wu, J. Li, and L. Guo, "A multi-stage attack mitigation mechanism for softwaredefined home networks," IEEE Trans. Consum. Electron., vol. 62, no. 2, pp. 200-207, 2016.

72. H.-C. Jang, C.-W. Huang, and F.-K. Yeh, "Design a bandwidth allocation framework for SDN based smart home," in Information Technology, Electronics and Mobile Communication Conference (IEMCON), 2016 IEEE 7th Annual, 2016, pp. 1-6.

73. R. M. Abuteir, A. Fladenmuller, and O. Fourmaux, "Sdn based architecture to improve video streaming in home networks," in Advanced Information Networking and Applications (AINA), 2016 IEEE 30th International Conference on, 2016, pp. 220-226.

74. J. Ruckert, R. Bifulco, M. Rizwan-Ul-Haq, H.-J. Kolbe, and D. Hausheer, "Flexible traffic management in broadband access networks using software defined networking," in Network Operations and Management Symposium (NOMS), 2014 IEEE, 2014, pp. 1-8.

75. H. H. Gharakheili, V. Sivaraman, A. Vishwanath, L. Exton, J. Matthews, and C. Russell, "SDN APIs and Models for Two-Sided Resource Management in Broadband Access Networks," IEEE Trans. Netw. Serv. Manag., vol. 13, no. 4, pp. 823-834, 2016.

76. K. J. Kerpez, J. M. Cioffi, G. Ginis, M. Goldburg, S. Galli, and P. Silverman, "Software-defined access networks," IEEE Commun. Mag., vol. 52, no. 9, pp. 152-159, 2014.

77. A. S. Thyagaturu, A. Mercian, M. P. McGarry, M. Reisslein, and W. Kellerer, "Software defined optical networks (SDONs): A comprehensive survey," IEEE Commun. Surv. Tutorials, vol. 18, no. 4, pp. 2738-2786, 2016.

78. R. G. Clegg, J. Spencer, R. Landa, M. Thakur, J. Mitchell, and M. Rio, "Pushing software defined networking to the access," in Software Defined Networks (EWSDN), 2014 Third European Workshop on, 2014, pp. 31-36.

79. S. S. W. Lee, K.-Y. Li, and M.-S. Wu, "Design and Implementation of a GPON-Based Virtual OpenFlow-Enabled SDN Switch,” J. Light. Technol., vol. 34, no. 10, pp. 2552-2561, 2016.

80. P. Gallo, K. Kosek-Szott, S. Szott, and I. Tinnirello, "SDN@ home: A method for controlling future wireless home networks," IEEE Commun. Mag., vol. 54, no. 5, pp. 123-131, 2016.

81. C. E. Rothenberg et al., "When open source meets network control planes," Computer (Long. Beach. Calif)., vol. 47, no. 11, pp. 46-54, 2014.

82. ISO, "ISO 9241-11, Guidance on usability." 1998.

83. ITU-T, "Recommendation Y.3001, Future Networks: Objectives and design goals," 2011. [Online]. Available: https://www.itu.int/rec/dologin_pub.asp?lang=s\&id=T-REC-Y.3001201105-I!!PDF-E\&type=items. [Accessed: 07-Nov-2017].

84. Open Networking Foundation, "NorthBound Interface (working group charter final v1.1)," 2013. 
[Online]. Available: https://www.opennetworking.org/images/stories/downloads/workinggroups/charter-nbi.pdf. [Accessed: 07-Nov-2017].

85. S. R. Chowdhury, M. Bari, R. Ahmed, and R. Boutaba, "Payless: A low cost network monitoring framework for software defined networks," in Network Operations and Management Symposium (NOMS), 2014 IEEE, 2014, pp. 1-9.

86. ITU-T, "Recommendation Y.2111, Resource and admission control functions in next generation networks," 2011. [Online]. Available: https://www.itu.int/rec/dologin_pub.asp?lang=e\&id=TREC-Y.2111-201111-I!!PDF-E\&type=items. [Accessed: 11-Nov-2017].

87. I. Bueno, J. I. Aznar, E. Escalona, J. Ferrer, and J. Antoni Garcia-Espin, “An opennaas based sdn framework for dynamic qos control," in Future Networks and Services (SDN4FNS), 2013 IEEE SDN for, 2013, pp. 1-7.

88. M. F. Bari, S. R. Chowdhury, R. Ahmed, and R. Boutaba, "PolicyCop: an autonomic QoS policy enforcement framework for software defined networks," in Future Networks and Services (SDN4FNS), 2013 IEEE SDN for, 2013, pp. 1-7.

89. Open Networking Foundation, "OpenFlow Management and Configuration Protocol: OFCONFIG 1.2 (ONF TS-016),” 2014. [Online]. Available: https://www.opennetworking.org/images/stories/downloads/sdn-resources/onfspecifications/openflow-config/of-config-1.2.pdf. [Accessed: 11-Nov-2017].

90. M. Dillon and T. Winters, "Virtualization of Home Network Gateways," Computer (Long. Beach. Calif)., no. 11, pp. 62-65, 2014.

91. Open Networking Foundation, "Relationship of SDN and NFV Issue 1 (ONF TR-518)," 2015. [Online]. Available: https://www.opennetworking.org/images/stories/downloads/sdnresources/technical-reports/onf2015.310_Architectural_comparison.08-2.pdf. [Accessed: 10Nov-2017].

92. J. Matias, J. Garay, N. Toledo, J. Unzilla, and E. Jacob, "Toward an SDN-enabled NFV architecture," Commun. Mag. IEEE, vol. 53, no. 4, pp. 187-193, 2015.

93. T. Kim, T. Koo, and E. Paik, "SDN and NFV benchmarking for performance and reliability," in Network Operations and Management Symposium (APNOMS), 2015 17th Asia-Pacific, 2015, pp. 600-603.

94. B. Han, V. Gopalakrishnan, L. Ji, and S. Lee, "Network function virtualization: Challenges and opportunities for innovations," IEEE Commun. Mag., vol. 53, no. 2, p. 90; 90-97; 97, 2015.

95. Intel, "White paper: OpenStack Enhanced Platform Awareness," 2015. [Online]. Available: https://01.org/sites/default/files/page/openstack-epa_wp_fin.pdf. [Accessed: 14-Nov-2017].

96. Software-Defined Networking Research Group (SDNRG), "Problem statement of SDN and NFV co-deployment in cloud datacenters (Internet-Draft)," 2016. [Online]. Available: https://tools.ietf.org/pdf/draft-gu-sdnrg-problem-statement-of-sdn-nfv-in-dc-01.pdf. [Accessed: 14-Nov-2017].

97. L. Qu, C. Assi, and K. Shaban, "Delay-Aware Scheduling and Resource Optimization with Network Function Virtualization," IEEE Trans. Commun., vol. 64, no. 9, pp. 3746-3758, 2016.

98. D. Krishnaswamy, R. Kothari, and V. Gabale, "Latency and policy aware hierarchical partitioning for NFV systems," in Network Function Virtualization and Software Defined Network (NFV-SDN), 2015 IEEE Conference on, 2015, pp. 205-211.

99. H. Chang, A. Hari, S. Mukherjee, and T. V Lakshman, "Bringing the cloud to the edge," in Computer Communications Workshops (INFOCOM WKSHPS), 2014 IEEE Conference on, 2014, pp. 346-351.

100. W. Shi and S. Dustdar, "The promise of edge computing," Computer (Long. Beach. Calif)., vol. 49, no. 5, pp. 78-81, 2016. 
101. P. Rygielski, M. Seliuchenko, S. Kounev, and M. Klymash, "Performance Analysis of SDN Switches with Hardware and Software Flow Tables," in Proceedings of the 10th EAI International Conference on Performance Evaluation Methodologies and Tools (ValueTools 2016), 2016.

102. L. Zhang, S. Wang, S. Xu, R. Lin, and H. Yu, "TimeoutX: An adaptive flow table management method in software defined networks," in Global Communications Conference (GLOBECOM), 2015 IEEE, 2015, pp. 1-6.

103. M. Kuźniar, P. Pereš’ \ini, and D. Kostić, "What you need to know about SDN flow tables," in International Conference on Passive and Active Network Measurement, 2015, pp. 347-359.

104. Software-Defined Networking Research Group (SDNRG), "Cooperating Layered Architecture for SDN (Internet-Draft)," 2016. [Online]. Available: https://tools.ietf.org/pdf/draft-irtf-sdnrglayered-sdn-01.pdf. [Accessed: 14-Nov-2017].

105. Interface to Network Security Functions (I2NSF), "Framework for Interface to Network Security Functions," 2016. [Online]. Available: https://tools.ietf.org/pdf/draft-ietf-i2nsf-framework04.pdf. [Accessed: 14-Nov-2017].

106. E.-D. Kim, S. Lee, Y. Choi, M.-K. Shin, and H.-J. Kim, "Evaluations of a multiple flow tables construction scheme for service function chaining," in Information Networking (ICOIN), 2016 International Conference on, 2016, pp. 424-426.

107. Home Gateway Initiative, "Parental control in the home v1.0 (HGI-GD003-R2)," 2008. [Online]. Available:

http://www.homegatewayinitiative.org/userfiles/file/downloads/HGI_Parental_control_v1.pdf. [Accessed: 04-Dec-2017].

108. D. Fernandez, F. J. Ruiz, L. Bellido, E. Pastor, O. Walid, and V. Mateos, "Enhancing Learning Experience in Computer Networking through a Virtualization-Based Laboratory Model," Int. J. Eng. Educ., vol. 32, no. 6, pp. 2569-2584, 2016.

109. FCC's Office of Engineering and Technology and Consumer and Governmental Affairs Bureau, “2014 Measuring Broadband America, Fixed Broadband Report.” 2014.

110. C. M. Lentisco, M. Aguayo, L. Bellido, E. Pastor, D. De-Antonio-Monte, and A. Garcia Bolivar, "A virtualized platform for analyzing LTE broadcast services," in Networks and Communications (EuCNC), 2015 European Conference on, 2015, pp. 512-516.

111. FCC's Office of Engineering and Technology and Consumer and Governmental Affairs Bureau, "2015 Measuring Broadband America, Fixed Broadband Report." 2015.

112. Cisco, "Cisco Network Services Orchestrator for Network Functions Virtualization (NFV)," White Paper, 2017. [Online]. Available:

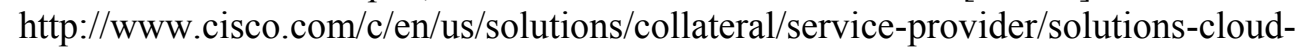
providers/white-paper-c11-738702.pdf. [Accessed: 18-Dec-2017]. 


\section{Apéndice A. Diagramas de Estado}

En este apéndice se muestran los diagramas de estado de los módulos que han sido desarrollados para la prueba de concepto tanto para la capa de Gestión como para la capa de Aplicación SDN.

\section{A1. Aplicación de Estado de la Red}

\section{- API REST}

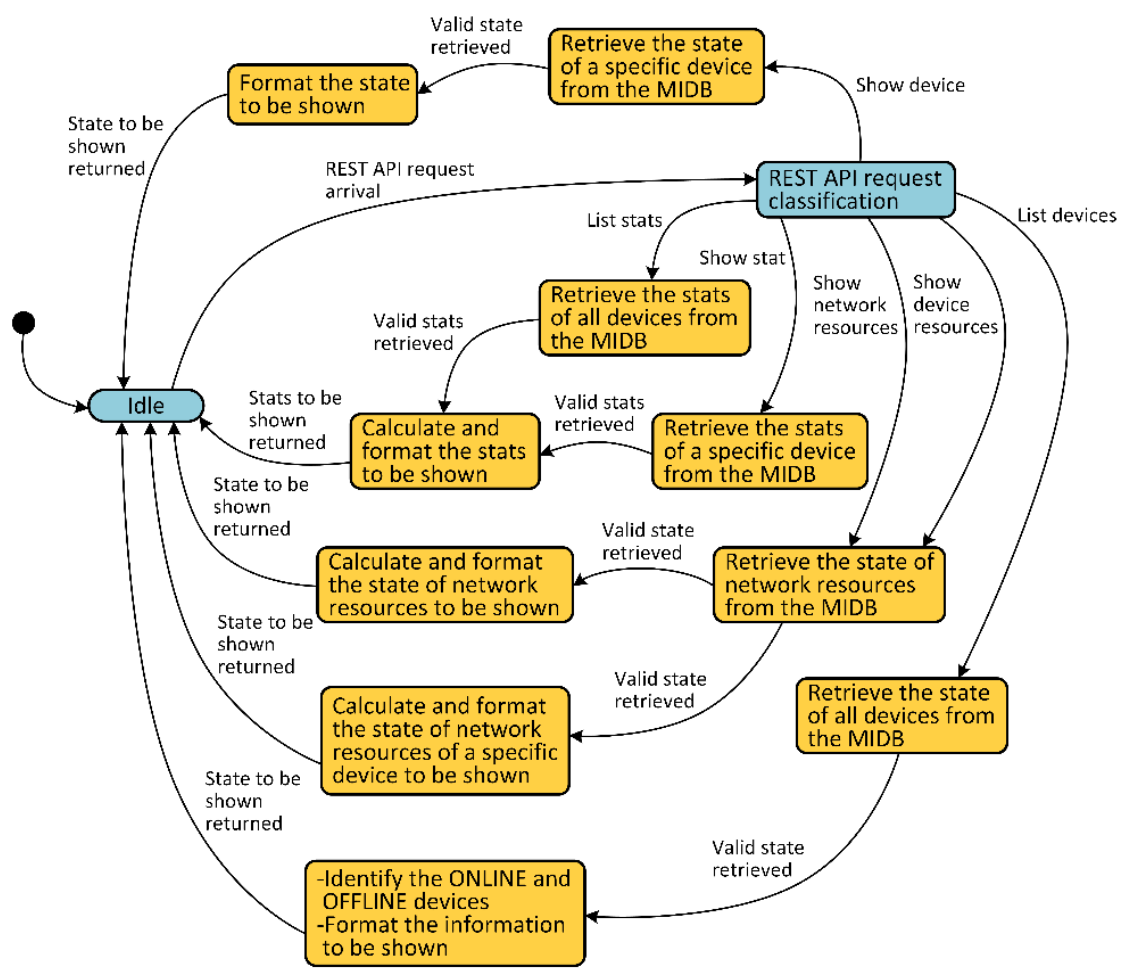

- Procesamiento de Mensajes RENESE

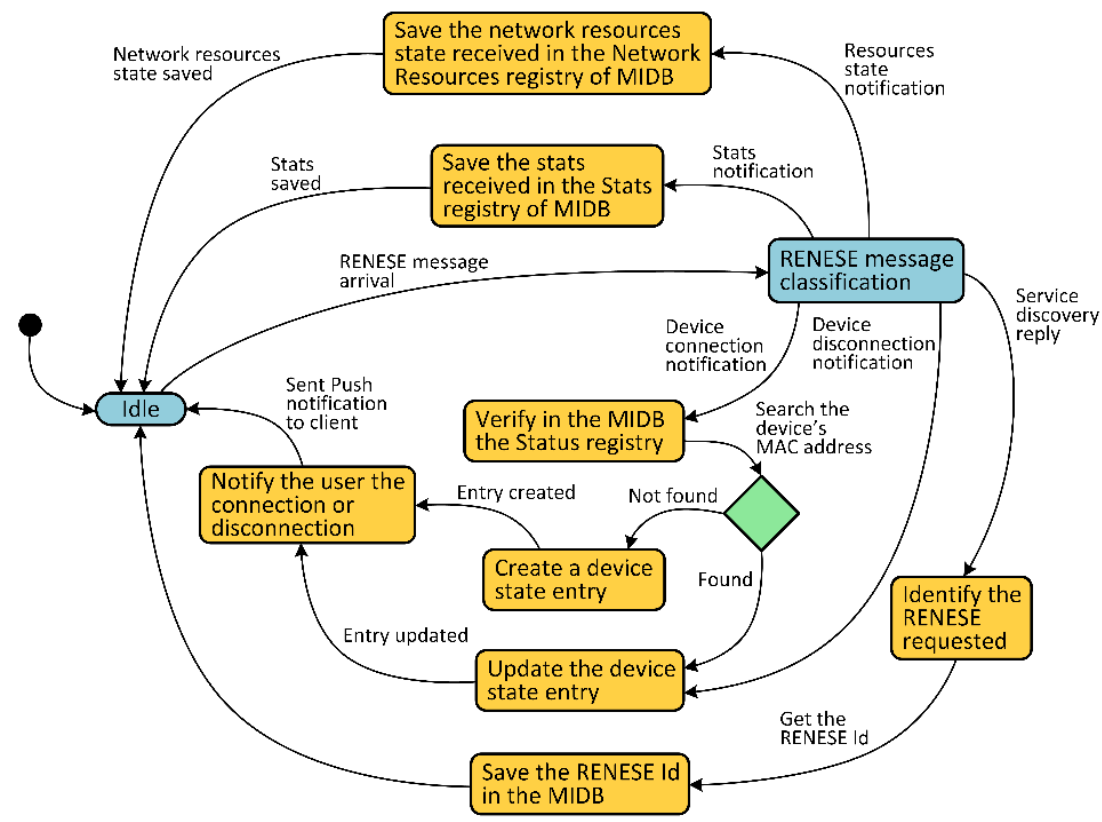




\section{A2. Servicio de Seguimiento de Dispositivos}

- Evento de conexión o desconexión

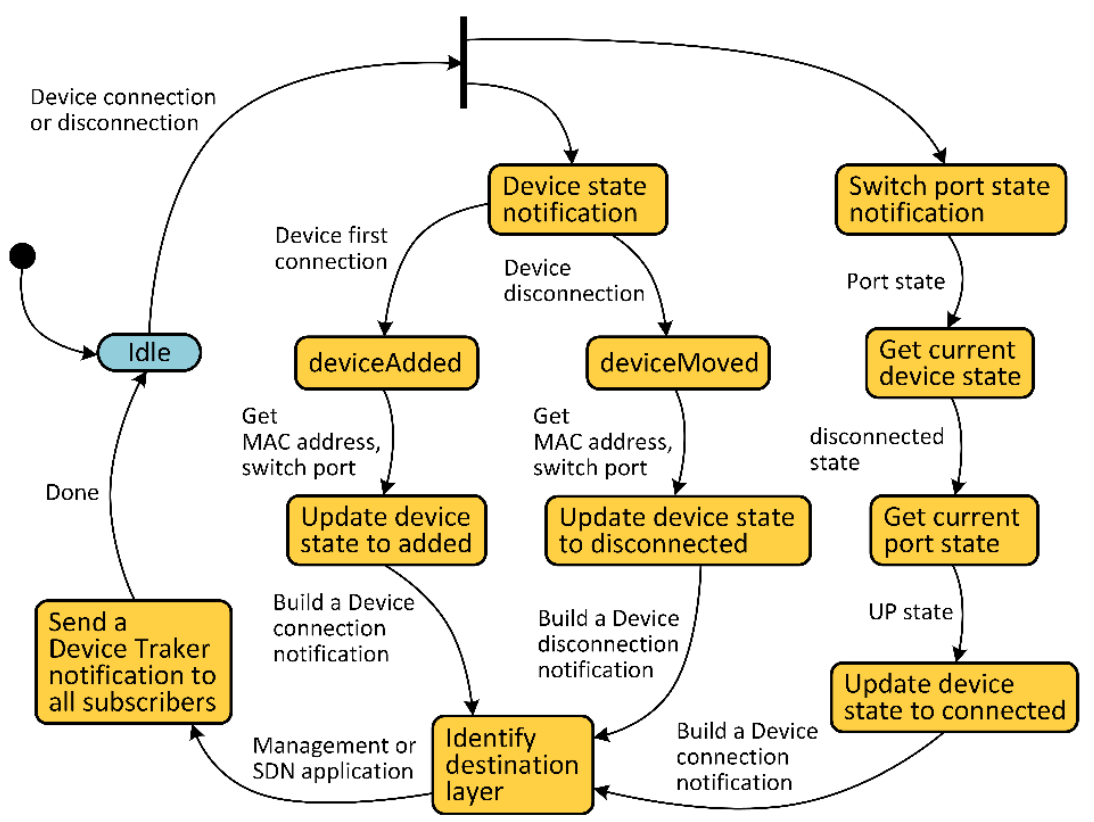

- Procesamiento de mensajes RENESE

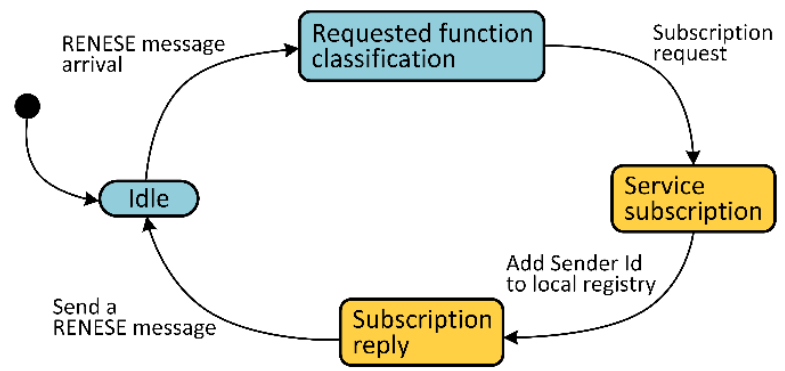

- Establecimiento del estado del puerto del switch (OVS)

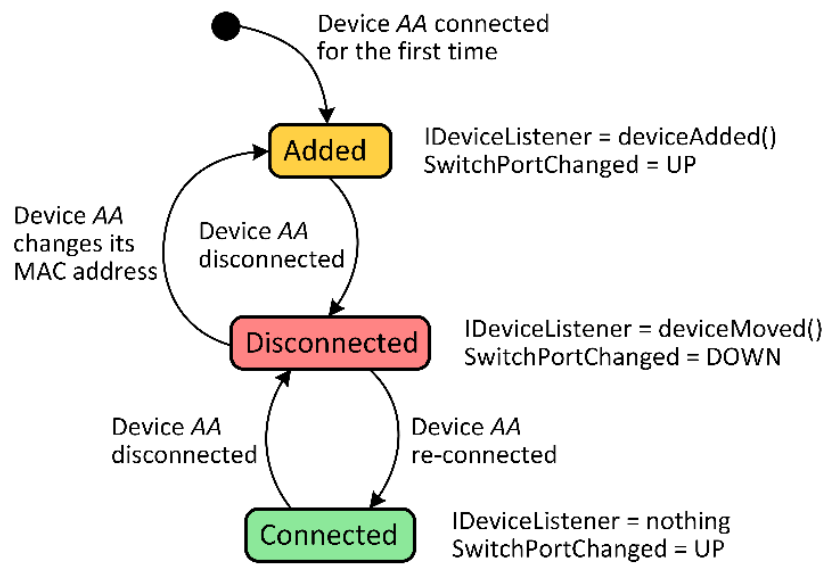




\section{A3. NERON}

- Procesamiento de mensajes RENESE

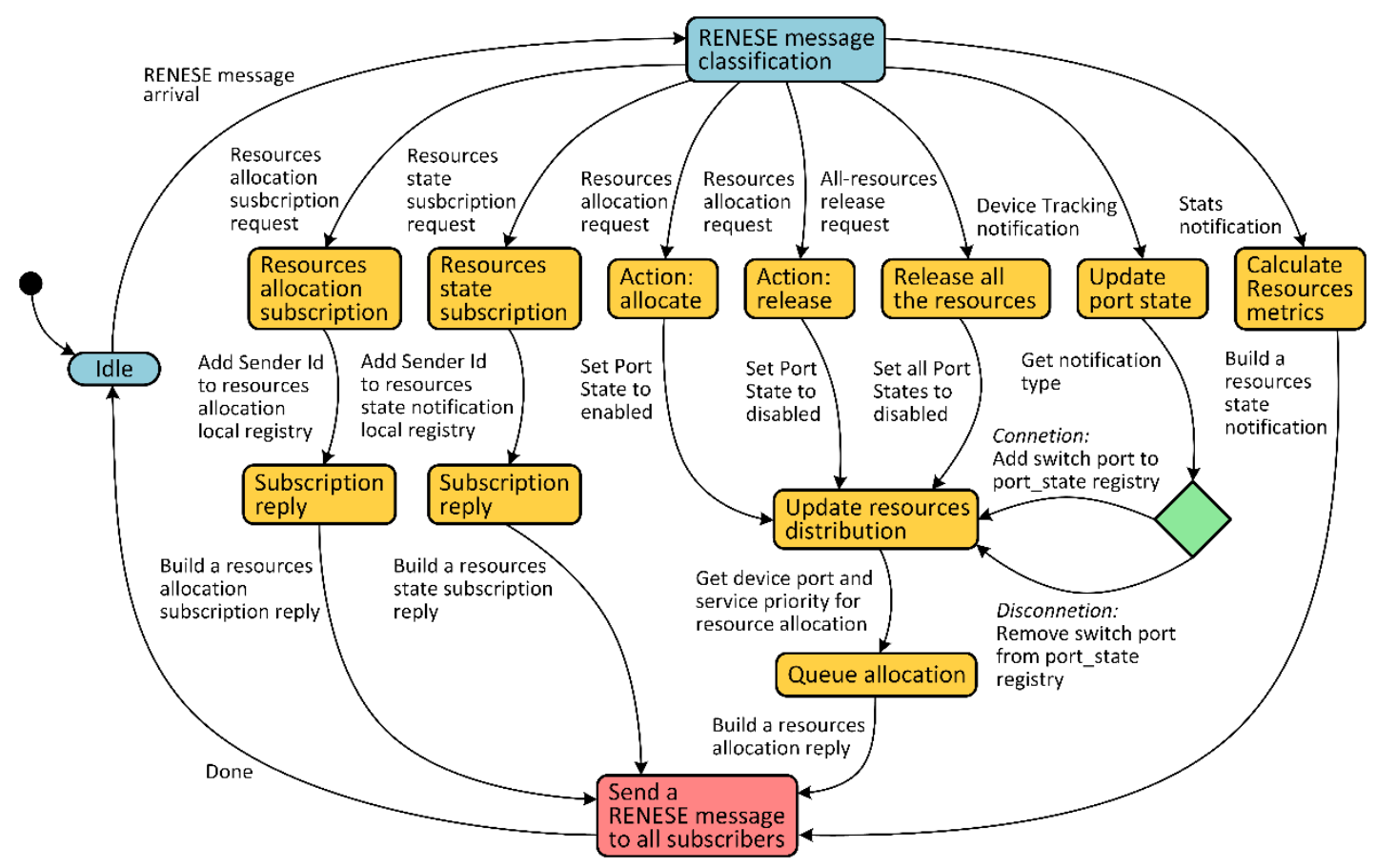

\section{A4. NESA}

\section{- Recolección de estadísticas de puerto y notificación}

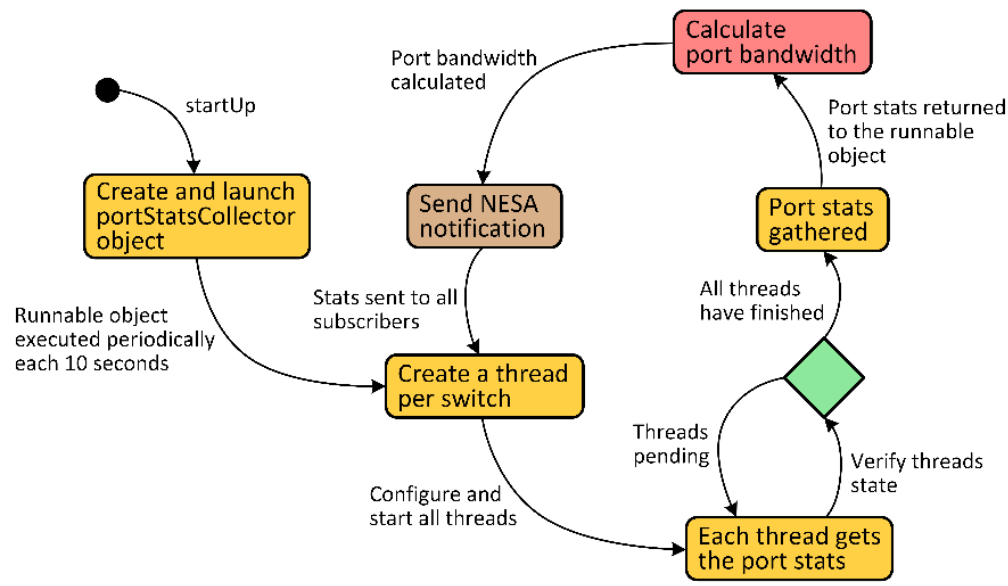

- Procesamiento de mensajes RENESE

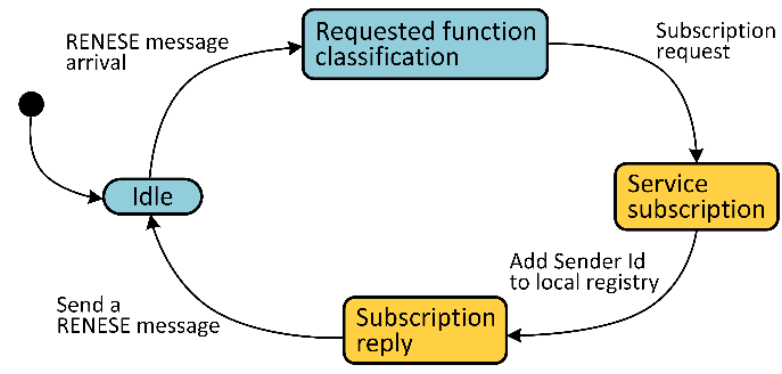




\section{A5. Gestor de aplicaciones RENEMA}

- Asignación de Id

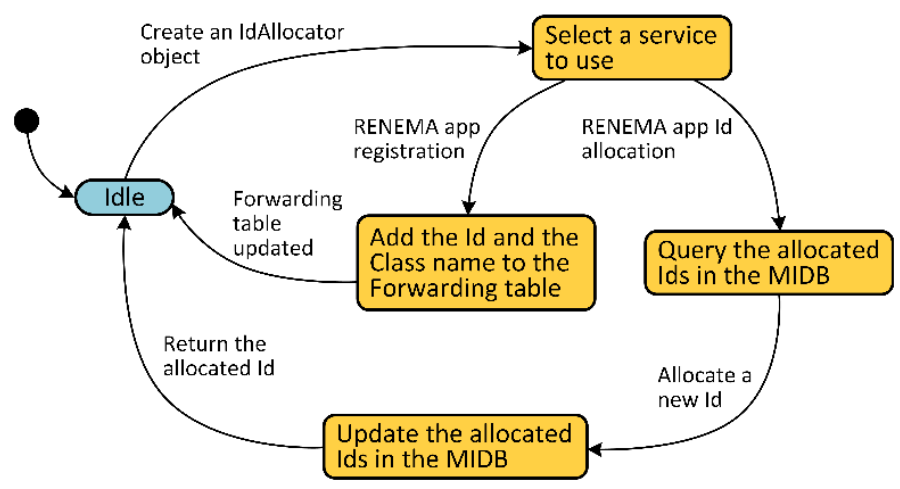

- Recepción de mensajes RENESE

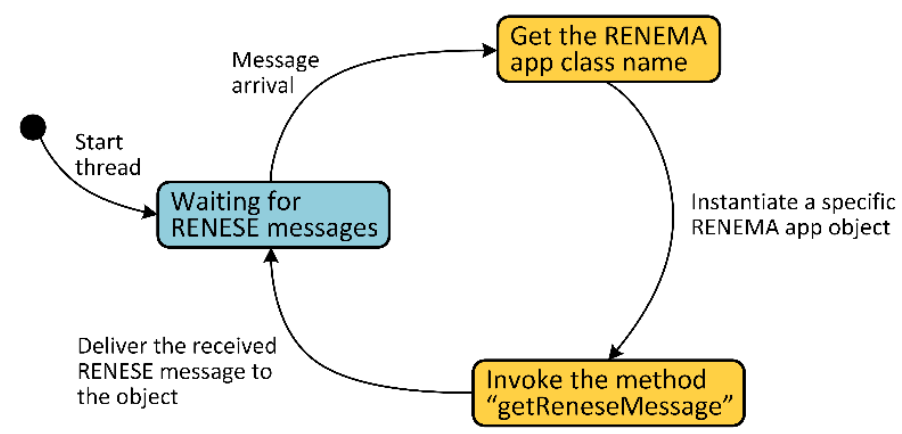

- Envío de mensajes RENESE

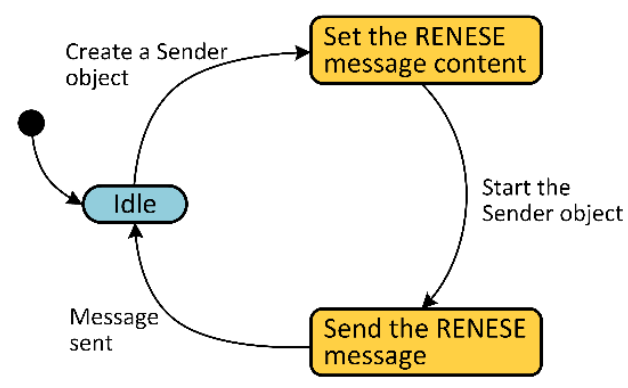

\section{A6. Gestor de RENESEs}

- Envío de mensajes RENESE

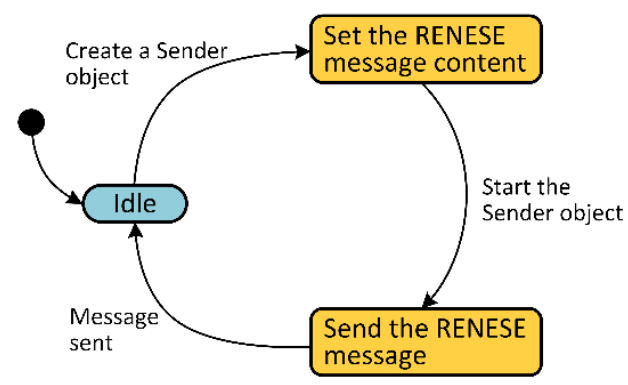




\section{- Recepción de mensajes RENESE}

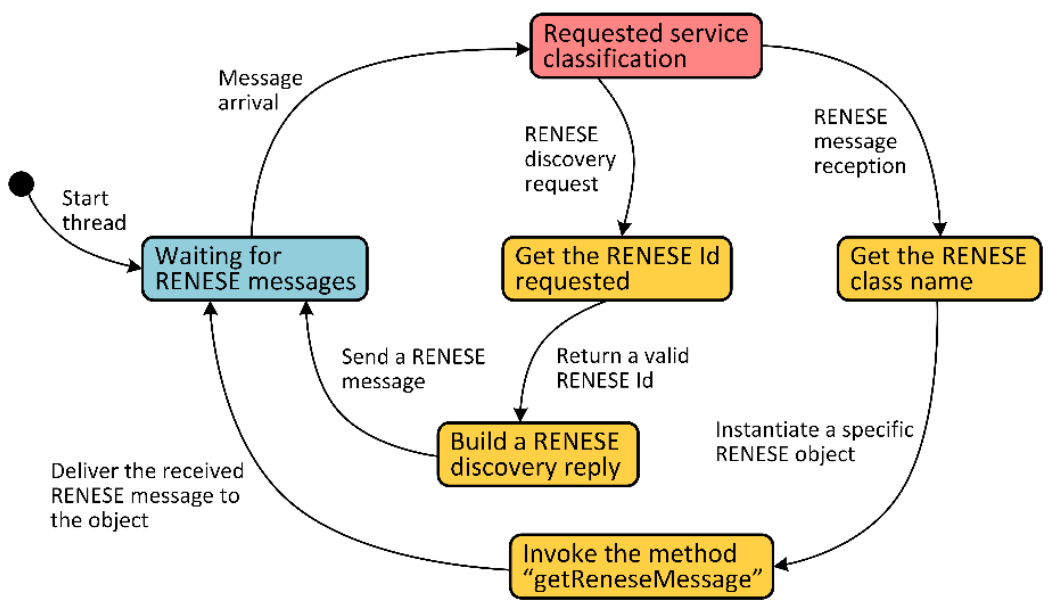

- Procesamiento de mensajes RENESE

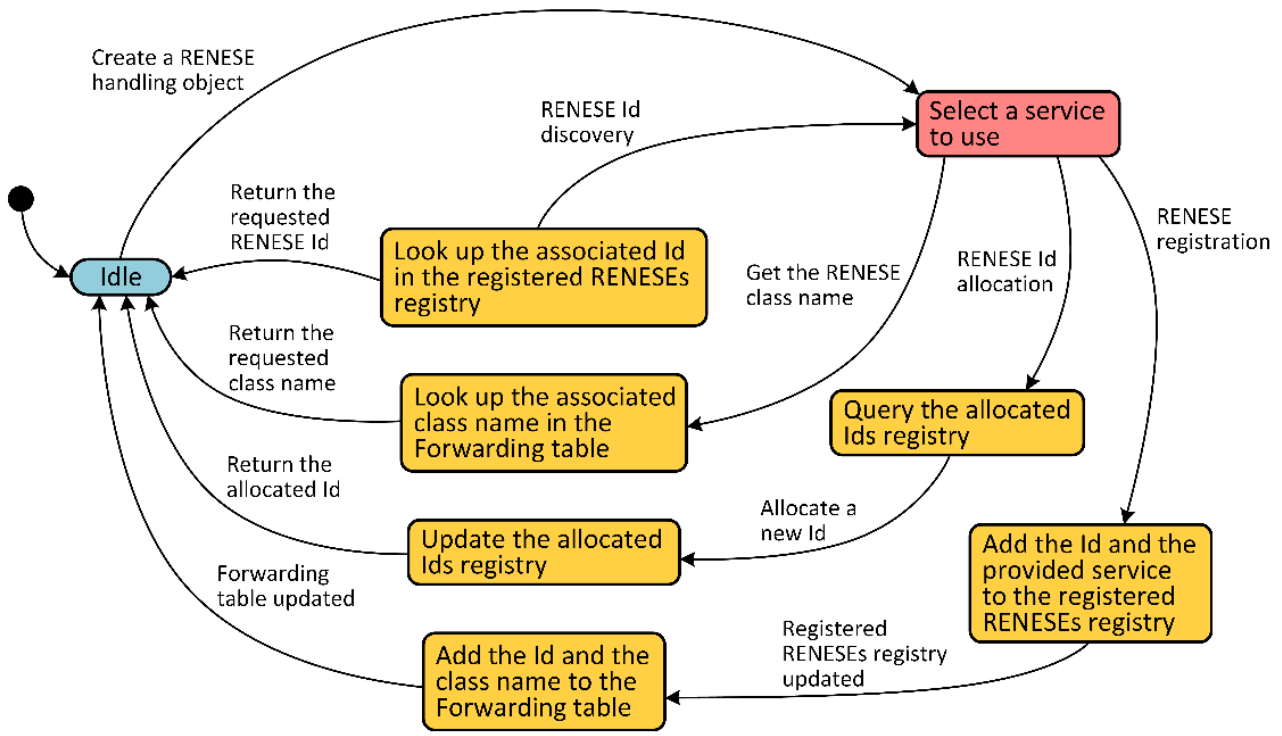

- Re-envío de mensajes RENESE

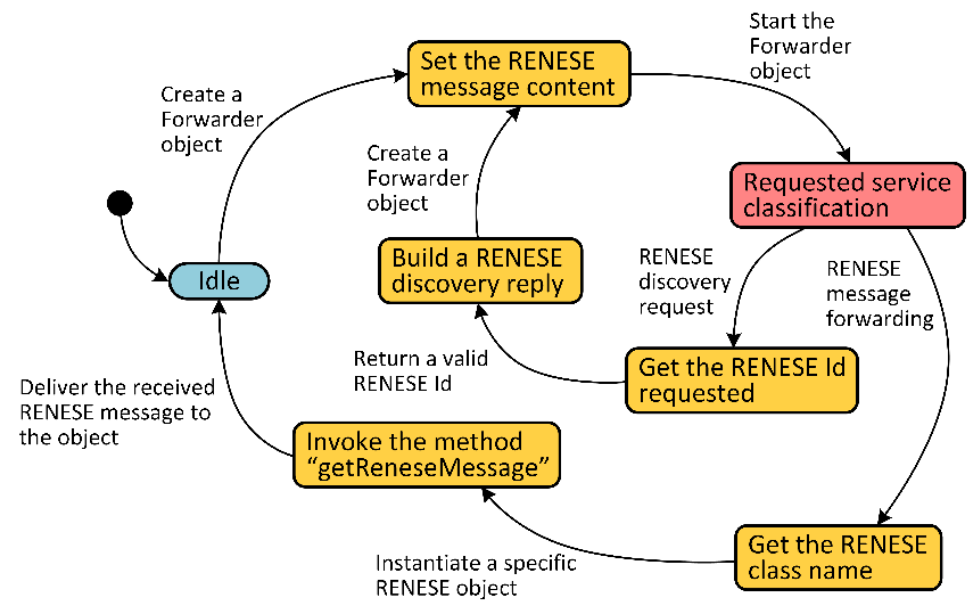




\section{Apéndice B. Descriptores de VNFs}

En este apéndice se muestran los descriptores adicionales que han sido definidos para implementar vMANDO como un servicio de red y proporcionar un tratamiento de tráfico diferenciado.

\section{B1. Descriptor para la VNF Management System}

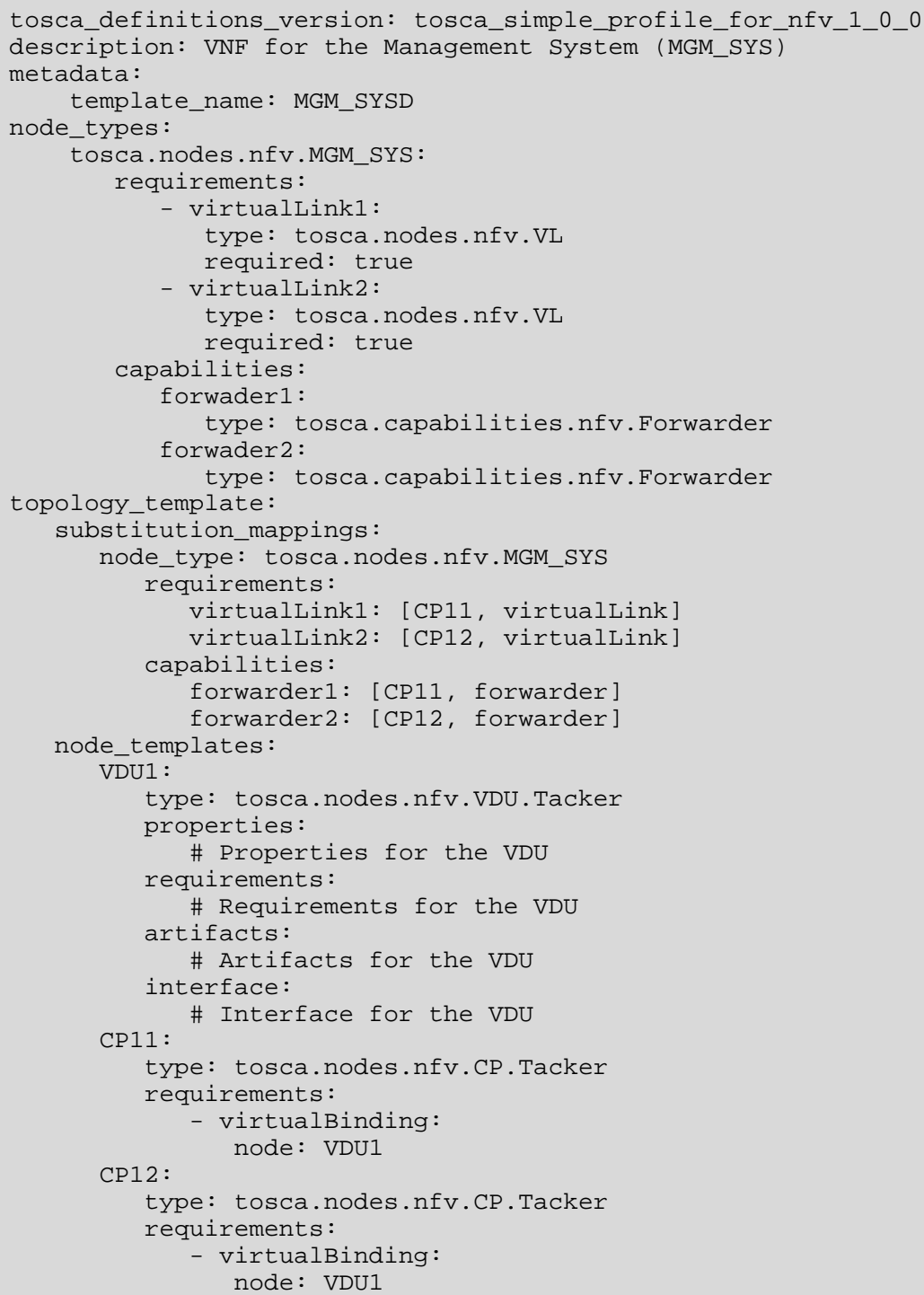




\section{B2. Descriptor para la VNF Classification and Shaping}

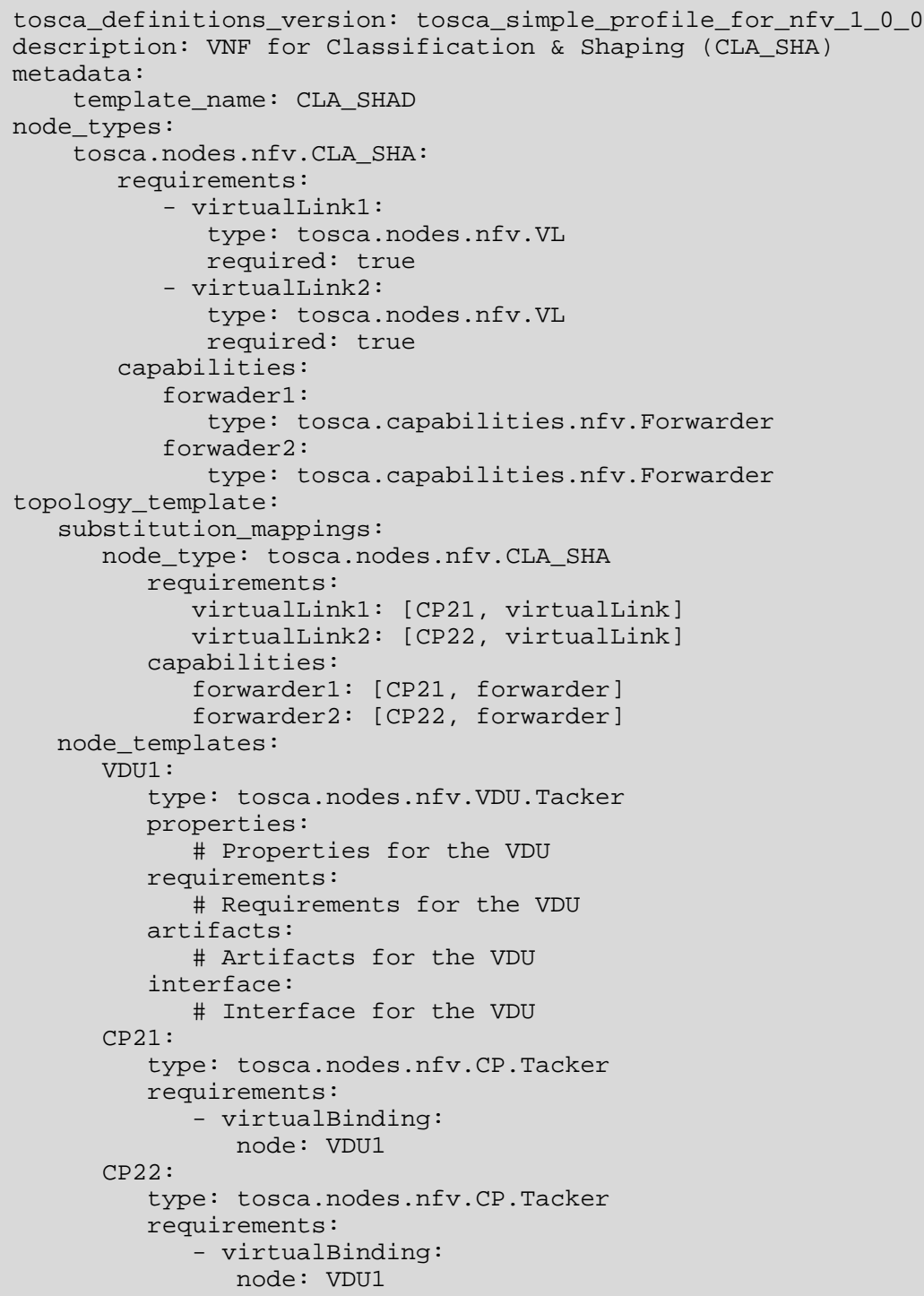




\section{B3. Descriptor para la VNF A}

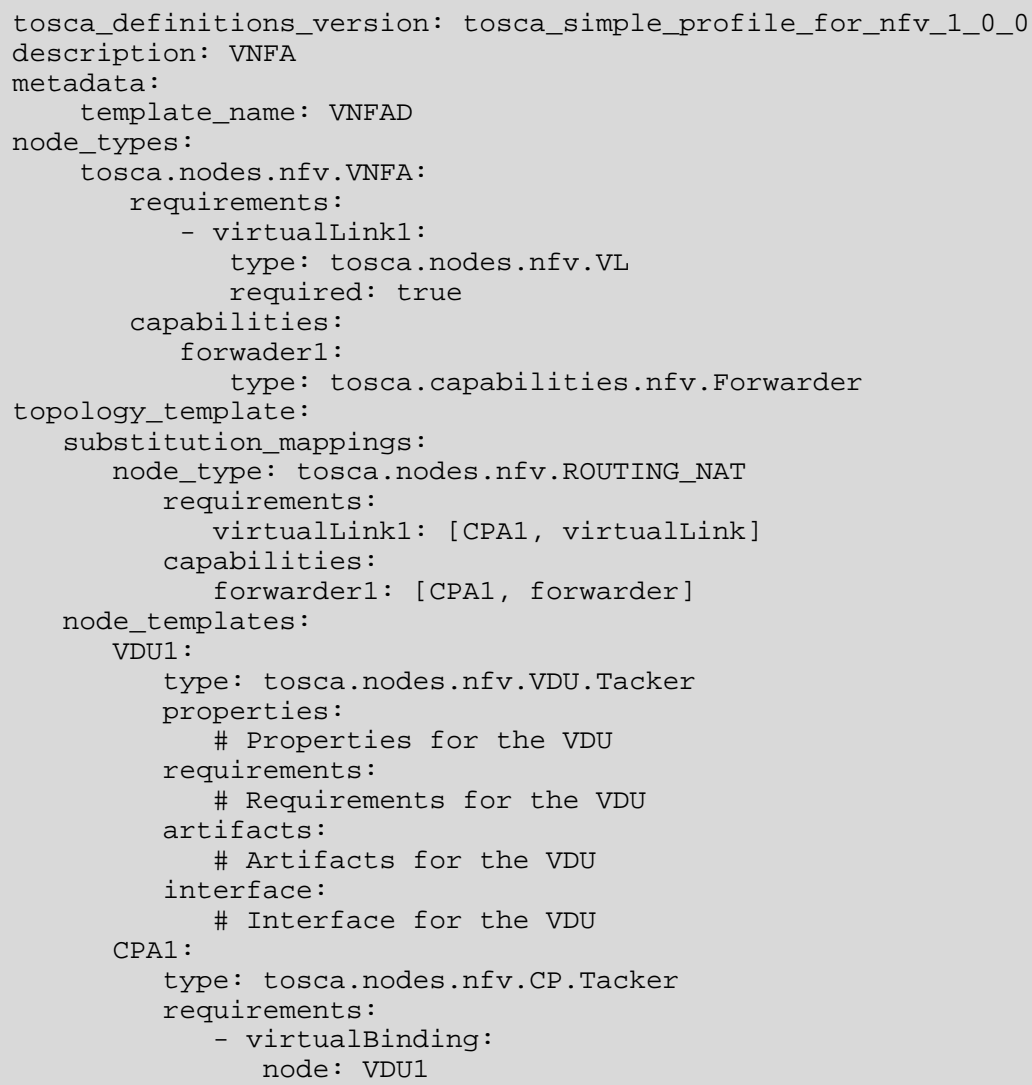

